\title{
The Effects of
}

Solar-geomagnetically Induced Currents on Electrical Systems in Nuclear Power Stations

Manuscript Completed: October 1993

Date Published: January 1994

Prepared by

M. Subudhi/Brookhaven National Laboratory

D. P. Carroll/University of Florida

S. Kasturi/MOS, Inc.

S. K. Aggarwal, NRC Program Manager

Brookhaven National Laboratory

Upton, NY 11973

Subcontractors:

University of Florida

MOS, Inc.

Prepared for

Division of Engineering

Office of Nuclear Regulatory Research

U.S. Nuclear Regulatory Commission

Washingtcn, DC 20555-0001

NRC FIN A3270

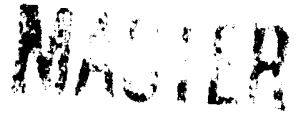




\begin{abstract}
This report presents the results of a study to evaluate the potential effects of geomagnetically induced currents (GICs) caused by the solar disturbances on the in-plant electrical distribution system and equipment in nuclear power stations. The plant-specific electrical distribution system for a typical nuclear plant is modeled using the ElectroMagnetic Transient Program (EMTP). The computer model simulates online equipment and loads from the station transformer in the switchyard of the power station to the safety-buses at 120 volts to which all electronic devices are connected for plant monitoring. The analytical model of the plant's electrical distribution system is studied to identify the transient effects caused by the half-cycle saturation of the station transformers due to GIC. This study provides results of the voltage harmonics levels that have been noted at various electrical buses inside the plant. The emergency circuits appear to be more susceptible to high harmonics due to the normally light load conditions. In addition to steady-state analysis, this model was further analyzed simulating various plant transient conditions (e.g., loss of load or large motor start-up) occurring during GIC events. Detail models of the plant's protective relaying system employed in bus transfer application were included in this model to study the effects of the harmonic distortion of the voltage input. Potential harmonic effects on the uniterruptable power system (UPS) are qualitatively discussed as well.
\end{abstract}




\section{CONTENTS}

$\underline{\text { Page }}$

ABSTRACT

iii

LIST OF FIGURES

vii

LIST OF TABLES

xi

SUMMARY

xiii

ACKNOWLEDGEMENTS

$\mathrm{XV}$

1. INTRODUCTION $\ldots \ldots \ldots \ldots \ldots \ldots \ldots \ldots \ldots \ldots \ldots \ldots \ldots \ldots \ldots \ldots$

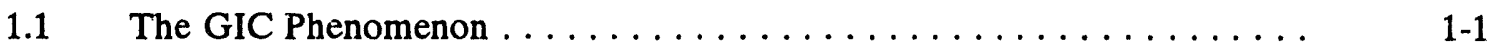

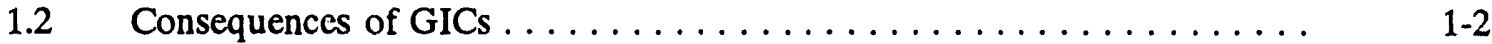

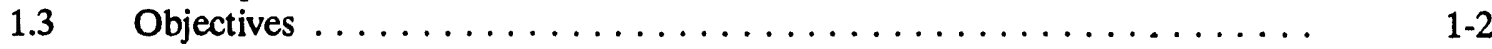

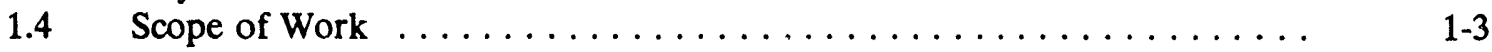

2. PROBLEMS ATTRIBUTED TO GICS .................. $2-1$

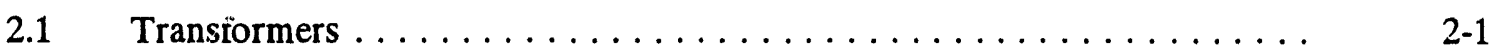

2.1.1 Localized Heating $\ldots \ldots \ldots \ldots \ldots \ldots \ldots \ldots \ldots \ldots \ldots . \ldots \ldots . \ldots \ldots$

2.1.2 Harmonics . . . . . . . . . . . . . . . . . . . . . .

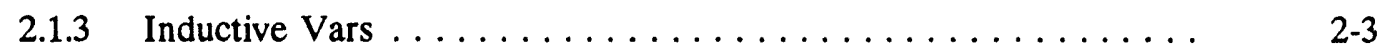

2.2 Current Transformers and Protective Relays ................ $2-4$

3. NUCLEAR EXPERIENCE $\ldots \ldots \ldots \ldots \ldots \ldots \ldots \ldots \ldots \ldots \ldots \ldots \ldots$

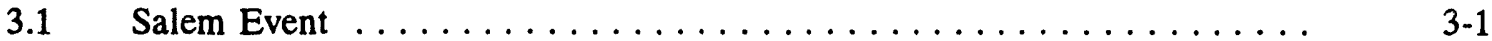

3.2 Hope Creek \& Bruce Events . . . . . . . . . . . . . . . . .

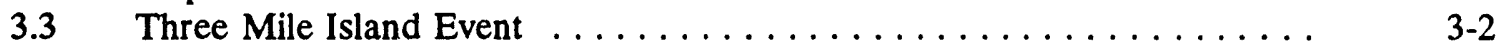

4. EMTP SIMULATION OF GIC EFFECTS $\ldots \ldots \ldots \ldots \ldots \ldots \ldots \ldots \ldots$

4.1 Preliminary Simulation of the Salem 1 Plant . . . . . . . . . . . . . . . $4-1$

4.2 Detailed Simulation of a Typical Generating Plant $\ldots \ldots \ldots \ldots \ldots \ldots . . . . .4$

4.2.1 Plant Description ...................... 4-3

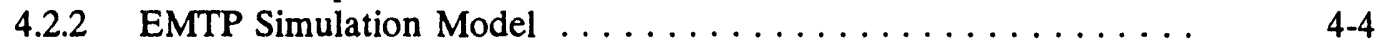

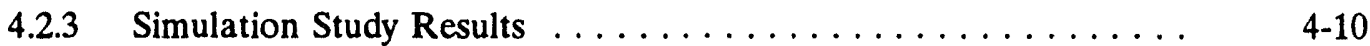

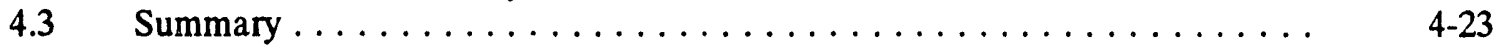

5. EFFECTS OF TRANSMISSION LINE CONFIGURATION $\ldots \ldots \ldots \ldots \ldots$

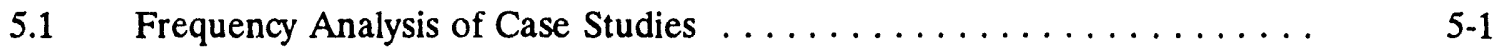

$5.2 \quad$ Extended Case Studies . . . . . . . . . . . . . . . . . . . . . $5-3$

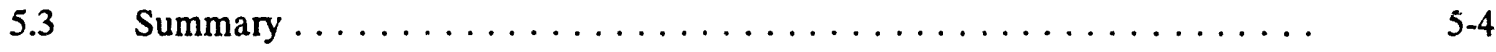


6. EFFECTS OF GIC HARMONICS ON PLANT EQUIPMENT $\ldots \ldots \ldots \ldots \ldots$

$6.1 \quad$ Harmonics in Power Systems . . . . . . . . . . . . . . . . . . . . . . $6-1$

6.2 Impact on Uninterruptible Power System . . . . . . . . . . . . . . . $6-2$

6.3 Impact of Harmonics on Protective Relays . . . . . . . . . . . . . $6-3$

6.3.1 Harmonics in Instrument Transformers . . . . . . . . . . . . . 6-5

6.3.2 GIC Effects on Relay and CT Performance .............. 6-6

6.3.3 Simulation of Harmonic Effects on Undervoltage Relays . . . . . . 6 6-6

$6.4 \quad$ Conclusions . . . . . . . . . . . . . . . . . . . . . . .

7. EFFECT OF TRANSIENT CONDITIONS ON GIC-INDUCED HARMONICS 7-1

$7.1 \quad$ Modified Simulation Studies $\ldots \ldots \ldots \ldots \ldots \ldots \ldots \ldots \ldots \ldots \ldots$

$7.2 \quad$ Simulation Results . . . . . . . . . . . . . . . . . . .

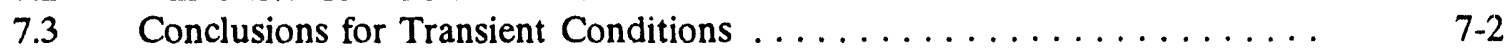

8. CONCLUSIONS AND RECOMMENDATIONS $\ldots \ldots \ldots \ldots \ldots \ldots \ldots \ldots$

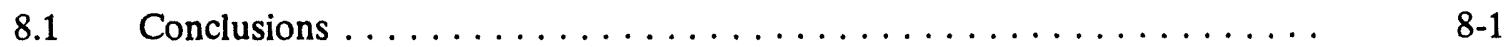

8.2 Recommendations for Future Work . . . . . . $\ldots \ldots \ldots \ldots \ldots \ldots$

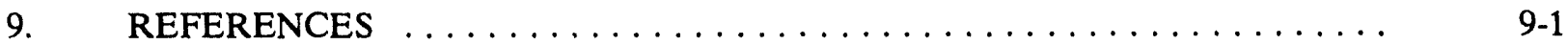

APPENDIX A: BACKGROUND ON SOLAR PHENOMENA $\ldots \ldots \ldots \ldots \ldots \ldots$ A-1

APPENDIX B: ELECTROMAGNETIC TRANSIENTS PROGRAM (EMTP) $\ldots \ldots \ldots \quad$ B-1

APPENDIX C: SAMPLE EMTP STUDIES $\ldots \ldots \ldots \ldots \ldots \ldots \ldots \ldots \ldots \ldots \ldots \ldots$

APPENDIX D: SALEM 1 SIMULATION AND SAMPLE RESULTS $\ldots \ldots \ldots \ldots \ldots$ D-1

APPENDIX E: MODEL DATA AND SAMPLE INPUT FILES

FOR EMTP SIMULATION $\ldots \ldots \ldots \ldots \ldots \ldots$ E-1

APPENDIX F: SIMULATION OUTPUT WAVEFORMS $\ldots \ldots \ldots \ldots \ldots \ldots \ldots$. F-1

APPENDIX G: FREQUENCY SCAN ANALYSIS $\ldots \ldots \ldots \ldots \ldots \ldots \ldots \ldots \ldots$ G-1

APPENDIX $\mathrm{H}:$ EXTENDED SIMULATION STUDIES $\ldots \ldots \ldots \ldots \ldots \ldots \ldots . \quad \mathrm{H}-1$ 


\section{LIST OF FIGURES}

$\underline{\text { Page }}$

1-1 ESP between grounded-wye transformer neutrals and resultant geomagnetically-induced currents

2-1 Half-Cycle saturation of a transformer core flux

4-1 System model for Salem 1 GIC simulation $\ldots \ldots \ldots \ldots \ldots \ldots \ldots \ldots$

4-2 Overall plant distribution $\ldots \ldots \ldots \ldots \ldots \ldots \ldots \ldots \ldots \ldots \ldots \ldots \ldots$

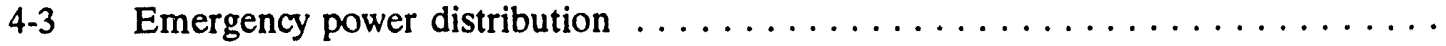

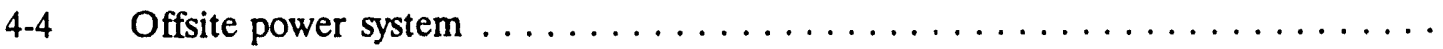

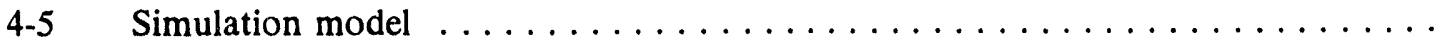

4-6 Simple model for distribution of GIC-induced harmonics between power station and transmission system

4-7 Frequency spectrum of generator step-up transformer magnetizing current

4-8 Frequency spectrum of reserve station service transformer magnetizing current

4-9 Frequency spectrum of phase A step-up transformer voltage on

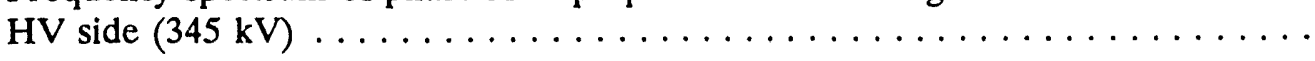

4-10 Frequency spectrum of phase A generator voltage $\ldots \ldots \ldots \ldots \ldots \ldots$

4-11 Frequency spectrum of phase A reserve station service

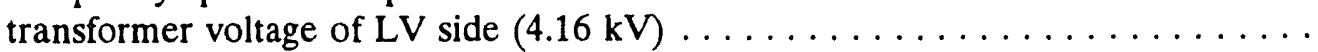

4-12 Frequency spectrum of $120 \mathrm{~V}$ emergency system voltage

4-13 Frequency spectrum for phase A generator voltage with 200 A GIC

4-14 Frequency spectrum for phase $A$ of reserve station service transformer voltage on $\mathrm{LV}$ side $(4.16 \mathrm{kV})-200 \mathrm{~A} \mathrm{GIC} \ldots \ldots \ldots \ldots \ldots \ldots$

4-15 Frequency spectrum of $120 \mathrm{~V}$ emergency system voltage with 200 A GIC

4-16 Frequency spectrum for step-up transformer magnetizing current with 500 A GIC 


\section{LIST OF FIGURES (Cont'd)}

4-17 Frequency spectrum for $120 \mathrm{~V}$ emergency system voltage with $500 \mathrm{~A} \mathrm{GIC}$

4-18 Comparison of THD for different GIC levels $\ldots \ldots \ldots \ldots \ldots \ldots \ldots$

4-19 Frequency spectrum of the magnetizing current in the reserve station service transformer, with separate $115 \mathrm{kV}$ line $\ldots \ldots \ldots \ldots \ldots$

4-20 Frequency spectrum of A-phase reserve station service transformer voltage of $4.16 \mathrm{kV}$ side, with separate $115 \mathrm{kV}$ line $\ldots \ldots \ldots \ldots \ldots \ldots$

4-21 Frequency spectrum of $120 \mathrm{~V}$ emergency system voltage, with separate $115 \mathrm{kV}$ line to reserve station service transformer $\ldots \ldots \ldots \ldots \ldots$.

4-22 Frequency spectrum of the A-phase magnetizing current in the reserve station service transformer, during start-up mode

4-23 Frequency spectrum for the A-phase reserve station service transformer voltage ( $4.16 \mathrm{kV}$ side), during start-up mode

4-24 Frequency spectrum of $120 \mathrm{~V}$ emergency system voltage, during

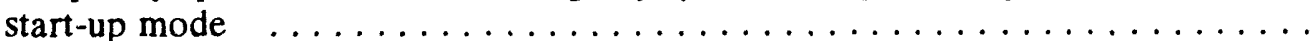

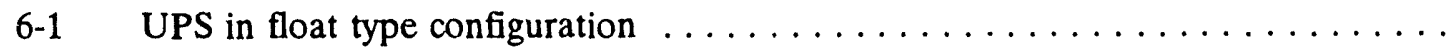

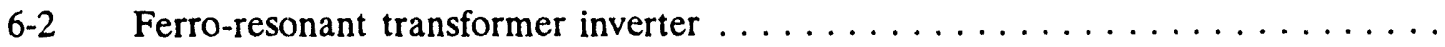

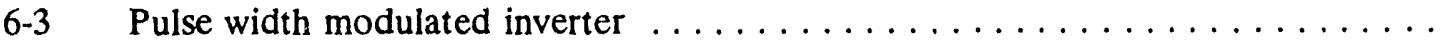

6-4 Basic circuit of GE Type NGV undervoltage relay $\ldots \ldots \ldots \ldots \ldots \ldots \ldots$

6-5 Block diagram of ABB Type ITE-27 undervoltage relay $\ldots \ldots \ldots \ldots \ldots \ldots$

6-6 Filtered and unfiltered voltages at $4.16 \mathrm{kV}$ side of reserve station service transformer during normal operation (100 A GIC) with separate $69.55 \mathrm{mi} 115 \mathrm{kV}$ line

6-7 Filtered and unfiltered voltages at $120 \mathrm{~V}$ emergency system bus during normal operation (100 A GIC) with separate $69.55 \mathrm{mi} 115 \mathrm{kV}$ line

6-8 Filtered and unfiltered voltages at $4.16 \mathrm{kV}$ side of reserve station service transformer during normal operation (without GIC) with separate $69.55 \mathrm{mi} 115 \mathrm{kV}$ line 


\section{LIST OF FIGURES (Cont'd)}

Page

6-9 Filtered and unfiltered voltages at $120 \mathrm{~V}$ emergency system bus during normal operation (without GIC) with separate $69.55 \mathrm{mi} 115 \mathrm{kV}$ line ........

6-10 Voltages on $4.16 \mathrm{kV}$ side of reserve station service transformer during normal operation (150 A GIC) with separate $50 \mathrm{mi} 115 \mathrm{kV}$ line .......

6-11 Frequency spectrum of phase A voltage on $4.16 \mathrm{kV}$ side of reserve station service transformer during normal operation (150 A GIC)

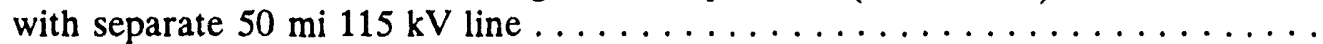

6-12 Normal and emergency system voltages at $120 \mathrm{~V}$ buses during normal operation $(150 \mathrm{~A} \mathrm{GIC})$ with separate $50 \mathrm{mi} 115 \mathrm{kV}$ line $\ldots \ldots \ldots \ldots \ldots$

6-13 Frequency spectrum of $120 \mathrm{~V}$ emergency system voltage during normal operation $(150 \mathrm{~A} \mathrm{GIC})$ with separate $50 \mathrm{mi} 115 \mathrm{kV}$ line $\ldots \ldots \ldots \ldots \ldots \ldots$

7-1 Reserve station service transformer magnetizing currents immediately after closing in $12000 \mathrm{Hp}$ motor load during start-up mode, with $50 \mathrm{~A} \mathrm{GIC}, 42.38 \mathrm{mi}$ line and remote source impedance.

7-2 Transient motor currents after closing in $12000 \mathrm{Hp}$ motor load during start-up mode, with $50 \mathrm{~A}$ GIC, $42.38 \mathrm{mi}$ line and remote

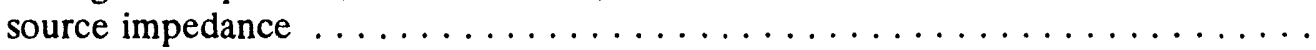

7-3 Reserve station service transformer $4.16 \mathrm{kV}$ voltages before closing in $12000 \mathrm{Hp}$ motor load during start-up mode, with $50 \mathrm{~A}$ GIC, $42.38 \mathrm{mi}$ line and remote source impedance

7-4 Reserve station service transformer A-phase transient voltage on $4.16 \mathrm{kV}$ side immediately after closing in $12000 \mathrm{Hp}$ motor load during start-up mode, with $50 \mathrm{~A}$ GIC, $42.38 \mathrm{mi}$ line and remote

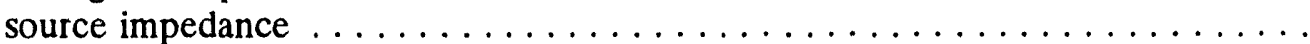

7-5 Reserve station service transformer $4.16 \mathrm{kV}$ voltages, $0.3 \mathrm{sec}$ after closing in $12000 \mathrm{Hp}$ motor load during start-up mode, with $50 \mathrm{~A} \mathrm{GIC}$, $42.38 \mathrm{mi}$ line remote source impedance

7-6 Normal and emergency system voltages at $120 \mathrm{~V}$ buses before closing in $12000 \mathrm{Hp}$ motor load during start-up mode, with $50 \mathrm{~A} \mathrm{GIC}, 42.38$ mi line and remote source impedance

7-7 Normal and emergency system voltages at $120 \mathrm{~V}$ buses, $0.3 \mathrm{sec}$ after closing in $12000 \mathrm{Hp}$ motor load, during start-up mode with $50 \mathrm{~A} \mathrm{GIC}$, $42.38 \mathrm{mi}$ line and remote source impedance 


\section{LIST OF FIGURES (Cont'd)}

$\underline{\text { Page }}$

7-8 Reserve station service transformer magnetizing currents immediately after tripping $12000 \mathrm{Hp}$ motor load during start-up mode, with $50 \mathrm{~A} \mathrm{GIC}$, $42.38 \mathrm{mi}$ line and remote source impedance $\ldots \ldots \ldots \ldots \ldots \ldots$

7-9 Reserve station service transformer $4.16 \mathrm{kV}$ voltages before tripping $12000 \mathrm{Hp}$ motor load during start-up mode, with $50 \mathrm{~A} \mathrm{GIC}, 42.38 \mathrm{mi}$

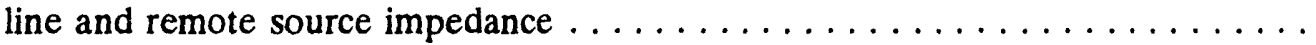

7-10 Reserve station service transformer $4.16 \mathrm{kV}$ voltages $0.3 \mathrm{sec}$ after tripping $12000 \mathrm{Hp}$ motor load during start-up mode, with $50 \mathrm{~A} \mathrm{GIC}$, $42.38 \mathrm{mi}$ line and remote source impedance 


\section{LIST OF TABLES}

Page

6-1 Comparison of Relay Data at $4.16 \mathrm{kV}$ Emergency Bus During Normal

Operation (100 A GIC) with Separate $69.55 \mathrm{mi} 115 \mathrm{kV}$ Line $\ldots \ldots \ldots \ldots \ldots$

6-2 Comparison of Relay Data at $120 \mathrm{~V}$ Emergency Bus During Normal

Operation (100 A GIC) with Separate $69.55 \mathrm{mi} 115 \mathrm{kV}$ Line ...........

6-3 Comparison of Relay Data at 4.16 kV Emergency Bus During Normal Operation (150 A GIC) with Separate $50 \mathrm{mi} 115 \mathrm{kV}$ Line . . . . . . . . .

6-4 Comparison of Relay Data at $120 \mathrm{~V}$ Emergency Bus During Normal Operation (150 A GIC) with Separate $50 \mathrm{mi} 115 \mathrm{kV}$ Line . . . . . . . . . . .

6-5 Comparison of Relay Data at $4.16 \mathrm{kV}$ Emergency Bus During Startup

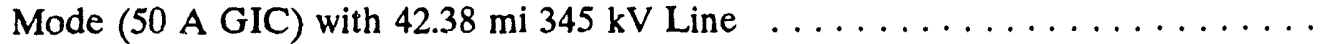

6-6 Comparison of Relay Data at 120 V Emergency Bus During Startup Mode (50 A GIC) with $42.38 \mathrm{mi} 345 \mathrm{kV}$ Line 


\section{SUMMARY}

Most previous analysis on the effects of geomagnetically induced currents (GIC) on electric utility systems has concentrated on steady-state phenomena, with the main interest in the generator step-up transformer and the off-site power system. This report investigates the possible effects that a GIC event might have on the power plant itself, by examining the harmonic distortion that could exist at various voltage levels in the on-site distribution system.

This study was carried out using an EMTP (ElectroMagnetic Transients Program) computer simulation of the electrical distribution system in a typical nuclear power plant. Elements of both the on-site and off-site power systems are included for completeness. The simulation employs detailed threephase models of all system components represented, including distributed-parameter transmission line models in the off-site system, saturation characteristics for main transformer, and rotating machine models of major pump loads. The simulation also models the essential features of both the nonsafetyrelated and the safety-related portions in the on-site distribution network.

Studies were performed for various operating configurations and conditions to determine harmonic levels in several parts of the distribution system. Results varied widely with station configuration and operating mode, but total harmonic distortion (THD) greater than $8 \%$ was observed in some low voltage buses. The emergency (safety-related) circuits appear to be more susceptible to high harmonics during normal station operation, due to their typically light loading conditions. Harmonic levels generally increased with escalating GIC, but not in a linear fashion, since the frequency spectrum of the transformer excitation current also shifts significantly with changing GIC. Very high GIC may actually reduce the higher order excitation harmonics, which in turn can reduce the THD in the distribution network. A major factor affecting the on-site harmonic levels is the high-voltage transmission line, since its harmonic impedance varies sharply with frequency, as determined by the line length and any external impedance. Certain line lengths can produce a very high impedance at one or more of the harmonics generated by transformer saturation, thus forcing large harmonic currents into the on-site network.

Work also includes refined load models, particularly at the lower voltage levels, in order to assess the effect of high harmonics on sensitive control circuits, such as protective relays. Study of transient conditions such as a loss of, or addition of a major load on $13.8 \mathrm{kV}$ bus (which is also fed by the reserve transformer) with high levels of harmonics due to GIC indicated that in and of itself, the transient event does not contribute to worsening the harmonic levels in the distribution system at the lower voltage levels. Indeed the addition of a major load improves the harmonic levels in the distribution system due to the increased transformer load as would be expected. However, even though the loss of a major load does not show any transient effect, it can result in a new steady state condition that results in increased harmonic levels throughout the distribution system. This also is expected because the transformer becomes lightly loaded.

Protective relays (both electro-magnetic and solid state relays) without a harmonic filter, which operated purely as a peak detector are vulnerable to false high readings resulting in improper operation even when only a 50A GIC is present in the system. However, the transmission network and plant electrical distribution system configurations can influence the extent of the net effect both positively and negatively. Relays that operate on average or rms values of the input waveforms are less vulnerable to mis-operation when exposed to bus conditions with a high harmonic levels. Addition of a harmonic filter can eliminate the problem completely. Based on this study, albeit limited in its scope, the one 
recommendation that can be made is that in undervoltage protective relays used to sense an undervoltagc or degraded voltage conditions for starting the on-site diesel generator, consideration should be given to installing an input side harmonic filter if one does not exist. 


\section{ACKNOWLEDGEMENTS}

The authors wish to express their appreciation to Mr. T.F. Schaub, Jr. who spent one semester at BNL from his academic year at SUNY, Buffalo and researched the subject from the available literatures. The background sections of the GMD events and their impact on station transformers are contributions from his preliminary study which he published as a BNL informal report.

Gratitude is also due to Bill Gunther and Mike Villaran for their valuable input to this effort. Patricia Ennis provided superb word processing and organizational skills to produce this report.

Review comments on the draft NUREG from Mr. John G. Kappenman of Minnesota Power, Bill Raughley and Mr. Jack E. Rosenthal of NRC, and Mr. M. E. Lapides of EPRI are greatly appreciated.

Finally, appreciation is due to NRC Program Manager, Satish Aggarwal, for his technical direction on this work. 


\section{INTRODUCTION}

\subsection{The GIC Phenomenon}

Transient disturbances in the earth's magnetic field, caused by the interaction of auroral currents (electrojets) with the earth's magnetic field, can induce electric potential gradients across the earth's surface. The auroral currents are created by solar phenomena through a complicated mechanism tersely outlined in Appendix A. The potentials, known as earth surface potentials (ESP), are due to portions of the earth's non-homogeneous surface being subjected to the time-varying magnetic field. ESP values can range from 1.2-6 V/km (2-10 V/mile). They act like quasi-dc voltage sources impressed between the grounded wye $(Y)$ neutrals of transformers at opposite ends of power transmission systems. The results of ESP are geomagnetically induced currents (GICs) - spurious, quasi-dc currents that can have magnitudes many times greater than the RMS ac exciting currents in transformer windings. In several research projects, GIC magnitudes greater than 100 A have actually been measured in transformers' neutral leads $[1,2]$.

There are three factors which combine to roughly determine the likelihood that any given power distribution system will experience GICs and its related effects. The first factor is the geological rock formation on which the transmission lines are situated. The resistance posed by igneous rock formations can be thought of as being in parallel with that of the power lines. Because igneous rock has a high resistivity, transmission lines situated on such formations, which have much lower resistances, will allow the GICs to flow through with greater ease [Fig. 1-1]. The latitude of the power lines is another factor which determines GIC susceptibility. Lines in the higher northern latitudes, closer to where the aurora appear, are more apt to be susceptible to GIC currents. Finally, the direction the power lines run determines the magnitude of GIC currents (1 to 10 ratio in E/W versus $N / S$ potential). If the previously mentioned electrojets are idealized as east-west line currents they will cause fluctuations, in turn, will induce an ESP in the east-west direction; thus, power lines running east-west may experience higher GICs than those running north-south due to a greater potential.

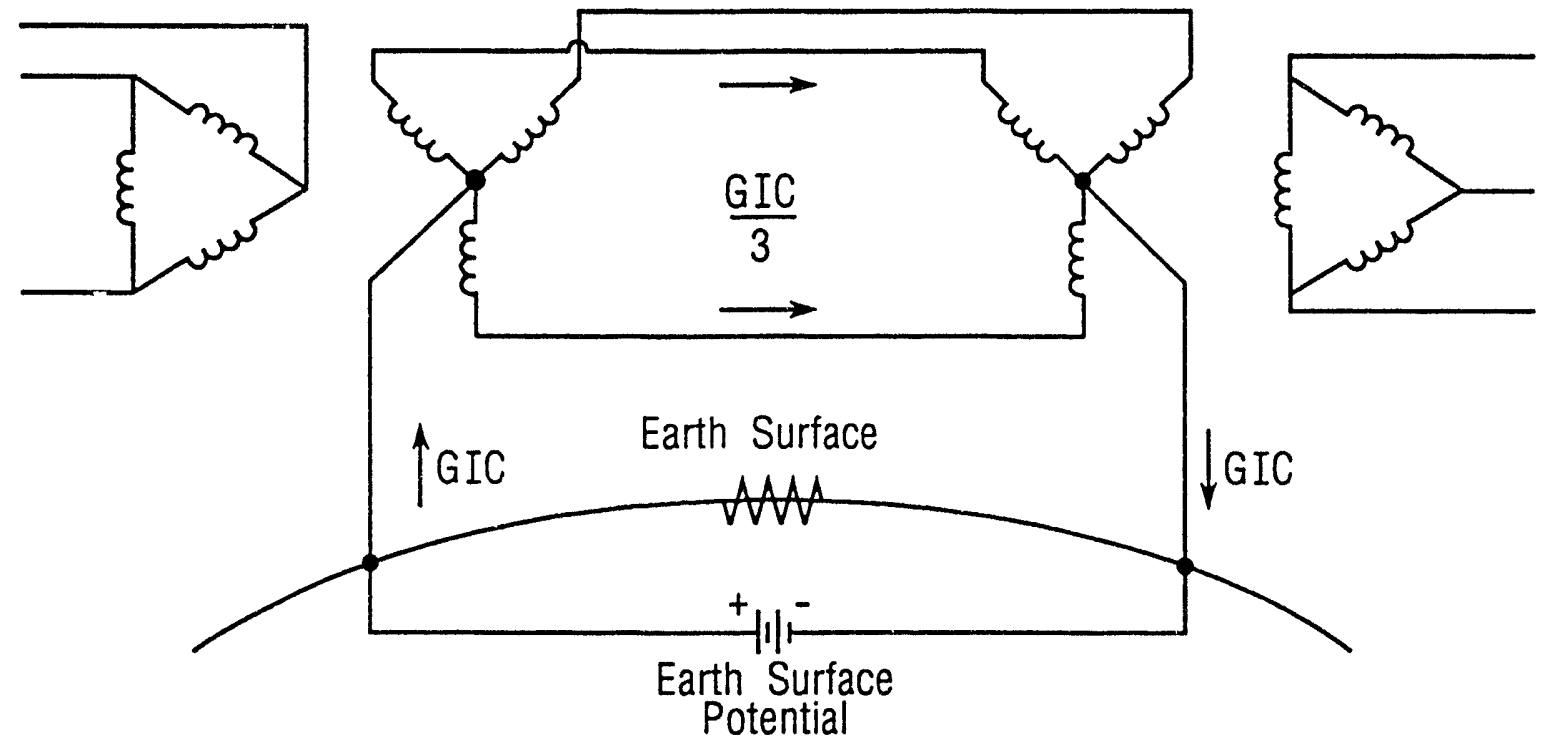

Fig. 1-1. ESP between grounded-wye transformer neutrals and resultant geomagnetically-induced currents. 
It must be understood that GICs are not pure dc, i.e., zero frequency. Calling GICs "quasi-dc" is meant to imply that they have extremely large periods, especially in comparison to conventional $60 \mathrm{~Hz}$ current. To demonstrate this claim an example is provided from Finland where research on GICs has been conducted since the late nineteen-seventies. One aspect of this research has included the measurement of GICs in transformers' grounded neutrals at various points of the Finnish $400 \mathrm{kV}$ grid. Fourier amplitudes that correspond to a sample of current measured at the Huutokoski station in southern Finland are studied. The lowest frequencies are the most important; the fundamental frequency of the current, which has the largest magnitude of all components is $260 \mu \mathrm{Hz}$. This means that $60 \mathrm{~Hz}$ current is roughly 231,000 times higher than this GIC example [2]. Typical GIC frequencies are in the $\mathrm{mHz}$ range, thereby justifying their treatment as quasi-dc.

\subsection{Consequences of GICS}

The primary effect GICs have upon transformers is known as half-cycle saturation. The consequences of half-cycle saturation are as follows:

- There is the potential for drastic stray leakage flux in the transformer which may cause excessive localized heating.

- The tiansformer becomes a rich source of even and odd harmonics.

- An increase in inductive vars is drawn by the transformer.

The stray-flax heating of transformers is an internal localized heating (ILH). ILH can cause accelerated aging of the transformer due to the thermal damage that is an immediate consequence of eddy currents from excessive flux. Physical damaging of the transformer will impede its ability to perform properly in context with the rest of the system.

The harmonics, for which the transformer is responsible, can affect the entire power transmission system in several ways, important examples include:

- Overloading of capacitor banks.

- Misoperating of relays.

- Causing of higher recovery voltages for circuit breakers.

The increase in inductive vars drawn by system transformers - like the harmonics produced - may also cause problems that are not confined to the transformers themselves, but are instead manifest throughout the power transmission system. Unusual swings in real and reactive power flow in transmission lines, and limitations on generators' var production capabilities are examples of such problems. Voltage collapses in the affected system is also a possibility (blackout).

The complications due to harmonic production and increased var demand demonstrate that concern must not be restricted solely to transformers [1] but should focus on a broader picture.

\subsection{Objectives}

Whether the effects of GIC are restricted to transmission systems or not, the potential - either directly or indirectly - for impact on a nuclear power station exists; e.g. a loss of offsite power is an example of how GICs can indirectly affect a nuclear power station. A great deal of concern was recently generated over an incidence at Quebec Hydro on March 13, 1989, since the entire province was blacked 
out as a result of GICs [3]. In total, 21,500 MW of generation was lost. Nine hours later, 17 percent of the province was still without service.

Occurrences at Salem Unit 1, Hope Cree Unit 1, and Three Mile Island Unit 1 have raised NRC's concern over the potential for trouble. It is expected that the climatic disturbances which cause GICs will reach their peak activity sometime between 1992 and 1995 (see Appendix A). Current trends are also indicating that this may be one of the highest peaks in decades.

The purpose of this report is: (1) to specifically examine GIC incidents at nuclear sites; (2) to investigate the manner in which GICs affect the plant electrical distribution system; (3) to quantify such GIC effects as manifested on the in-plant electrical distribution system buses at different voltage levels; and finally ( 4 to evaluate the impact of GIC on certain vital equipment connected to the plant electrical distribution system.

\subsection{Scope of Work}

A typical electrical distribution system inside a nuclear power plant is modeled by using the ElectroMagnetic Transient Program (EMTP), developed by the Bonneville Power Administration (BPA), an agency of the U.S. Department of Energy. A detailed description of the analytical capability of this transient program is given in Appendix B, followed by a number of applications presented in Appendix C. The purpose of these two appendices is to provide the reader some validation of the use of EMTP in modeling various electrical systems and components. Parametric studies of the subject plant model were performed to simulate the GIC effects on electrical buses at all voltage levels, including the 120 volt AC safety instrument bus. Detailed models of the two commonly used undervoltage protective relaying system were analyzed to investigate the effects of the harmonic distortion on the bus. 


\section{PROBLEMS ATTRIBUTED TO GICS}

\section{$2.1 \quad$ Transformers}

GIC enters and exits power systems through the grounded neutral connections of Wye connected transformers. GICs introduce a dc bias in the transformer core flux, i.e., apply a magnetic flux bias, leading to half-cycle saturation as shown in Figure 2.1 below.

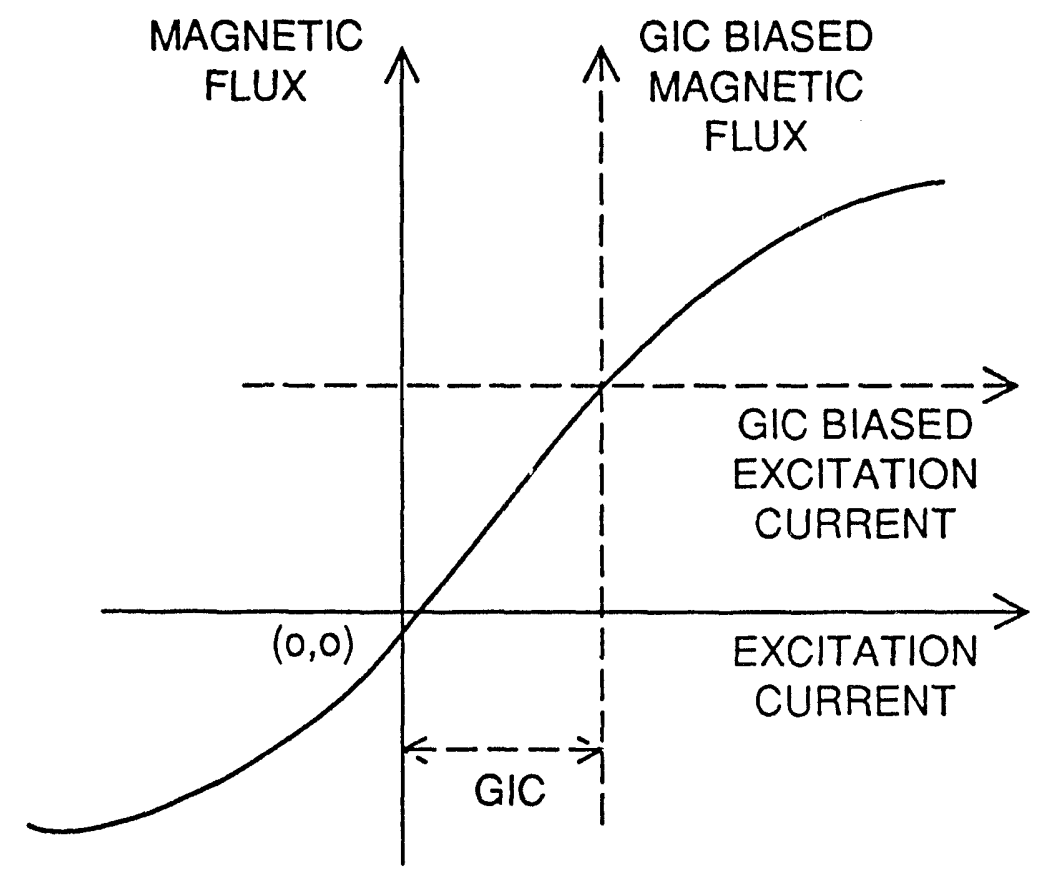

Fig. 2-1. Half-Cycle saturaticn of a transformer core flux.

Nearly all of the power system problems from GIC have been attributed to the half-cycle saturation of power transformers. Many studies, field measurements and tests conducted on transformers have established that half-cycle saturation of the transformers can result in:

(a) localized heating of transformers,

(b) the transformer becoming a rich source of even and odd harmonics, and

(c) a great increase in inductive vars drawn by the transformer.

\subsubsection{Localized Heating}

GICs result in simultaneous ac and quasi dc excitation of the transformer. Increased excitation currents in the transformer increase the magnetic intensity $\mathrm{H}$ in the Core. An increased magnetic intensity $(\mathrm{H})$ will attempt to raise the flux density $(\mathrm{B})$, but because of the core's ferromagnetic properties (see Appendix A for more details) it will become saturated, i.e., increasing $\mathrm{H}$ will not proportionally increase B. Once the core has become saturated, the flux will find its return path through the core 
surface into nearby structural members or windings of the transformer. Note that flux goes through the core - but a lot goes through non-core volume.

To draw an analogy between electric circuits and magnetic circuits, magnetic flux would be the counterpart of electric current. Qualitatively, conductivity is a measure of a material's tendency to allow current to flow. A good insulator, such as air, has a conductivity about $10^{-20}$ times that of copper. In magnetic circuits permeability is the measure of a material's tendency to allow flux to flow. Even a good magnetic insulator, a diamagnetic material such as bismuth, has a permeability only as large as $10^{4}$, and often as little as $10^{2}$ times that of a typical ferromagnetic material used in transformers' cores. Permeability $(\mu)$ can be defined as the slope of the B-H curve at any point, or $\mathrm{dB} / \mathrm{dH}$. Because of the non-linear saturation characteristic of transformers, the permeability of the material under scrutiny will be much less after saturation than it was before; there may, in fact, be only a very small difference between the permeabilities of the iron core and that of air. Thus, a high amount of flux may exit the transformer's core to enter nearby structural members thereby causing eddy currents to circulate in unlaminated materials. A changing flux impinging on an electrically conducting surface will cause these circulating or eddy currents (Faraday's Law). Such currents will tend to subject the material to Joule heating loss and, possibly, thermal degradation.

One EPRI study [4] categorized heating losses into four regions within the transformer:

- Eddy and hysteresis losses on the bulk material within the body of the core.

- Eddy loss on the surface of the core.

- Eddy loss in the structural member holding the core together.

- Winding losses.

The severity of loss in the first category is determined primarily by the intrinsic properties of the core material, in conjunction with the design of the core. Eddy loss on the surface of the core is caused by the radial component of leakage flux; this category of loss is normally present in a properly operating transformer but will be greatly increased due to saturation. Eddy loss in the core clamp is due solely to the entrance of leakage flux from the core. Winding losses are due both to high exciting current $\left(I^{2} R\right.$ loss) and leakage flux. The contribution from the leakage flux may be more significant however; within the first few windings, EPRI found that leakage losses were approximately 3 times as large as the $I^{2} R$ losses [4].

A loaded transformer always has a small amount of leakage flux that will have been taken into account in the design of the transformer. The losses due to leakage flux will be minimized by providing the transformer with laminated structural members or insulation where necessary. When the core saturates, an additional component of flux outside the core is created. This component may not have been taken into account by the designer because it does not occur in normally defined operation. The leakage flux may now enter members not prepared to minimize losses, and Joule heating will abound due to the eddy currents. In some incidents, thermal degradation - visible by discoloration of paint - on transformers' tanks has been attributed to the eddy currents caused by leakage flux which in turn is due to GICs [5].

McNutt classified transformer designs according to the sensitivity of particular core arrangements to GICs. EPRI's report, as well as others, confirms that various core designs pose different sensitivities to saturation caused by GICs. The ranking which follows, in order of decreasing sensitivity, was reached in [6]: 
1. Most sensitive

(a) Single-phase; shell form

(b) Single-phase; core form

2. Sensitive

(a) Three-phase; shell form, 7-leg core

(b) Three-phase; shell form, conventional

(c) Three-phase; core form, 5-leg core

3. Least sensitive

(a) Three-phase; core form, 3-leg core

\subsubsection{Harmonics}

Normally, the exciting current wave form is composed solely of odd-harmonic components due to the symmetry of the magnetization curve [see Appendix A for more details]. Because of the flux offset, resulting from GIC, symmetry about the origin no longer exists. As a result, the exciting current contains both even and odd components. The harmonic component, composition of the exciting current, varies depending on the magnitude of GIC current. The composition also depends on other parameters inherent in the transformer's design.

There is an important contrast between the exciting current profile of the single-phase transformer and the three-phase transformers. When a single-phase transformer is subjected to large magnitudes of GIC, the amount of a particular harmonic present is inversely proportional to the order of that harmonic; this is not so in thi: case of three-phase transformers.

The exciting current in three-phase transformers is a much more complicated pattern of sequence components when the transformer is saturated, i.e., there is an exciting current phase imbalance during saturation, which is caused by the magnetic asymmetry present in many three-phase transformers. Certain regions of the core may saturate before others, whereas with a single-phase core the entire core is either saturated or not. Since the three-phase transformers have magnetic paths that are common to more than one phase, analysis of magnetic circuit behavior is rather complicated when nearly equal amounts direct current are injected into each phase, with performance dependent on the specific core configuration [7].

\subsubsection{Inductive Vars}

The generator to which the transformer is directly connected will be rated for a given power output. The power can be split between active and reactive power, but because its magnitude is limited, increased demand for reactive power will decrease the supply of active or real power. Overall power for ac circuits is measured in volt-amperes, active or real power is measured in watts, and reactive power is measured in volt-amperes reactive (var). Hence, increasing the var requirements will decrease wattage.

Many definitions of "reactive power" exist from which to choose one. Walling and Khan [7] recommend separate treatment of fundamental and harmonic reactive power during distorted conditions because only the fundamental lagging current will have a significant impact on the system voltage profile, and because this is consistent with analysis tools used by the utilities for analyzing the voltage profile. Harmonic and unbalanced fundamental components are also important however, but are best treated separately. 
When the magnitude of GIC is increased, the exciting current wave profile is changed drastically. In particular, the fundamental frequency exciting current component greatly increases in magnitude, although by percentage it contributes less. This change is significant because the transformer acts as a reactive load, and by increasing the magnitude of the exciting current, especially that of the fundamental component, the transformer requires more reactive power.

The net result of the increased var demand leads to system-wide decreases in bus voltages. These voltage fluctuations contribute to degradation on system operation in several ways. A list given by Albertson, Kappenman, et al. [8] included the following consequences of increased var requirements: generator var output increases substantially, total system generation decreases, total system load decreases, system transmission losses are effected, and tie-line transfer capabilities are reduced.

\subsection{Current Transformers and Protective Relays}

Current Transformers (CT) are usually selected to limit operation on a very small and linear portion of the CT's saturation characteristics. Similar to the effect on power transformers, GIC tends to offset the normal operating point. Depending upon the magnitude of the GIC, the combined ac and $\mathrm{dc}$ flux can cause the CT to operate in the non-linear region of the CT's saturation characteristic, leading to distorted output. Also because, GICs can last several minutes, after a CT has been exposed to GIC, current interruption occurring at a higher flux level can result in a large trapped flux or remanence. Kappenman, et. al. [9] show that remanence increases with increasing GIC magnitudes. Higher remanence can affects transient performance.

Similarly operation of protective relays may be affected due to the interactions with the harsh harmonic environment. The relays may experience undesired operation, be de-sensitized, or may not operate at all. 


\section{NUCLEAR EXPERIENCE}

\subsection{Salem Event}

The Salem nuclear power station in New Jersey has experienced difficulties due to GIC currents. During the severe geomagnetic storm of March 13, 1989 three single-phase, shell form transformers were damaged. Later that year, in September, thermal damage was sustained by another transformer unit at Salem. From VAR readings made on the supply side of the transformer it was inferred that the magnitude of GIC in the high voltage windings during the first incident was roughly 60 amperes. Additionally, the transformers were fully loaded on March 13th., a fact which may have exacerbated damages.

The transformers involved in the March 13 th event were the 24-500 kV step-up transformers connected to the main generators. The internal "post-mortem" inspection revealed that extreme overheating had occurred in the LV bushing leads and the series connections between winding subgroups. Copper droplets were found to have issued from one lead. Accompanied by this was the production of acetylene gas; the releases of gas occurred during the fusion process when arcing between strands chemically decomposed the insulatio:. oil. All of these events occurred within a few minutes of GIC activity. The overheating which occurred at Salem Unit 2 in September was not as severe [6].

The conditions of the single-phase transformers in service at Salem experienced on March 13, 1989 indicated GIC in the HV winding equal to $4.8 \%$ of the rated load current. The result is a core which is saturated for almost 60 electrical degrees, thereby producing a magnetic current in the LV winding having a peak value of 6685 amperes and an rms value of 1980 amperes, or $13.2 \%$ of the rated load current [6].

Many main power transformers - such as those at Salem - usually employ oil as an insulator and coolant. The oil surrounds the core and windings of the transformer, and a surrounding tank structure then encases all of this. Electrical faults in transformers are invariably accompanied by the generation of a variety of gases as a result of the local chemical breakdown of the surrounding insulating material. After the VAR recordings at Salem had showed abnormally high amounts of current in the high voltage windings, gas chromatography tests were performed on the insulating oil of the transformers. Analysis of the dissolved gases present in the oil showed that high concentrations of many gases were present. Most alarming was the high concentration of acetylene which indicating that electrical arcing had taken place.

One of the virtues of the gas chromatography test is its ability to narrow down, or pinpoint, the type of electrical fault occurring within the transformer. Gases such as hydrogen, ethane, and ethylene require low-energy input to be released; thus, their presence may indicate that overheating or corona discharges have taken place. For a gas such as acetylene to be released, a high-energy input is needed, so that its presence would indicate that arcing had probably taken place [10]. After the high amount of gases in the transformers at Salem clearly indicated that a malfunctioning of some sort had occurred, the transformers were taken off-line and opened for visual inspection.

\subsection{Hope Creek \& Bruce Events}

Hope Creek nuclear power station is owned and operated by Public Service Electric and Gas Co. (PSE\&G) and Atlantic City Electric Co. It is a single unit, BWR located on the southern part of an 
artificial island on the east bank of the Delaware River in Lower Alloways Creek Township, Salem County, NJ - 30 miles southwest of Philadelphia, PA, and seven and a half miles southwest of Salem, NJ.

On March 13, 1989 the generator negative sequence current protective alarm operated many times. The cumulative time that the alarm operated was 6 minutes, 46 seconds. The General Electric relays were set to 0.05 pu generator rating. The generator appears to have eluded any heat damage from the negative sequence currents.

Negative sequence relays can be connected in the primary supply of the transformer and set as sensitively as required to protect against secondary phase-to-ground or phase-to-phase faults. The relays measure generator phase currents from which a single phase quantity is derived in the negative sequence filter. The filter produces an output signal proportional to the negative sequence component of the input current. Generator protection schemes have no specifically designed mechanism or relay to protect from harmonic currents, although some negative sequence relays will respond to harmonics in a manner not well understood. For example, at the Bruce nuclear generating station - a pressurized heavy water (PHWR) Candu-designed reactor, located in southern Ontario - RL type negative sequence relays alarmed while units with RC filter type relays did not. Newer negative sequence relays, which have band pass filters, are not expected to respond to harmonic distortion.

The relays at Bruce which did alarm were protecting 960 MWA generators and, like those at Hope Creek, were set at 0.05 pu of the generator rating, or $0.2 \mathrm{~A}$ at the Bruce station. All told, four negative sequence relays alarmed for two minutes apiece. The excessive amounts of negative sequence current detected is created by the saturated transformer, and almost immediately thereafter, found in the stator windings of the generators connected to them. These excessive negative sequence currents can cause overheating in the generator. This phenomena is not fully understood, but preliminary studies by one manufacturer suggest that the heating impact on the rotor face increases roughly in proportion to the square root of the rotor harmonic order. Thus, for example, $4 \%$ symmetrical 7 th-order harmonic content is predicted to have the same heating impact as $10 \%$ fundamental frequency negative sequence current [5].

\subsection{Three Mile Island Event}

Also on the infamous day in March 1989, another nuclear power station exhibited misoperations attributable to GICs. The problem - the tripping of capacitor banks, reportedly due to the high harmonic content of the current - occurred in Three Mile Island's $500 \mathrm{kV}$ substation.

The capacitor banks were $Y$ grounded, and electrically connected to transformer buses. The protection system which tripped the banks was a conventional unbalance scheme using a current transformer (CT) in the capacitor bank neutral circuit. This system is intended to detect blown capacitor fuses which is normally indicative of failed individual capacitor cans, manifested by $60 \mathrm{~Hz}$ neutral unbalance current. Usually the $60 \mathrm{~Hz}$ current in the neutral circuit is very low, but when one or more of capacitor fuses has opened the reactance of the remaining cans, and hence the voltage applied to the surviving cans increases. The relay is necessary to ensure that the applied voltage does not get so high as to damage any of the remaining capacitors.

This basic unbalance scheme will not only respond to excessive $60 \mathrm{~Hz}$ current but will also respond to system unbalancing influences and to harmonics. Normally the peak value of the exciting current is small enough to be neglected when considering the current circulation in a fully loaded transformer. When a transformer is saturated due to GICs however, the exciting current increases greatly 
in magnitude and harmonic composition and may distort the load current. This distortion of the load current on the secondary side which caused the relay to alarm. Thus, it is not GICs themselves that flow in the capacitor's neutral circuit - capacitors are a high impedance path to quasi-dc GIC - it is the harmonic current due to the saturated transformers.

Susceptibility to capacitor trips appears to be dependent on three factors:

- The capacitor's connection to ground.

- The level of harmonics in the local area.

- The capacitor protection's harmonic sensitivity.

There are relays which are desensitized to harmonics and thus appear to be the relay of choice at locations where the saturation of nearby transformers may cause unnecessary trips. Although a neutral unbalance relay may respond to harmonic currents. it is not suitable as protection for a neutral current overload, as its response to triple harmonics is uncertain at best - a $60 \mathrm{~Hz}$ relay is simply not designed to be reliable for a $180 \mathrm{~Hz}$ problem [5].

Finally it should be mentioned that the types of problems facing relay protection systems fall into two categories: those by which CT saturation due to GICs produces a distorted response for the protective device; and those stemming from the interaction between the harsh harmonic environment and the relay device. The tripping of the capacitor banks falls under the second category, i.e., the CT is responding accurately. Relay engineers should be aware that the possibility of CT saturation exists and may create additional problems for protection systems. 


\section{EMTP SIMULATION OF GIC EFFECTS}

\subsection{Preliminary Simulation of the Salem 1 Plant}

In order to investigate the basic parameters of GIC effects in nuclear plants, a preliminary investigation was carried out using a simplified model of the Salem 1 plant. This is a system well-known for suspected GIC interactions and offers previously documented data and simulations for comparison and verification of EMTP.

Figure 4-1 shows a one-line diagram of the portion of the Salem - New Freedom system simulated using EMTP. It is a three-phase simulation, which consists of the Salem 1 generator, the deltawye step-up transformer, and a major $500 \mathrm{kV}$ tie-line to the New Freedom bus. The Salem 1 generator is represented by a simplified voltage-behind-subtransient reactance, which was estimated to be $0.3 \mathrm{pu}$. The transformer was modeled in full detail with the excitation branch on the low voltage delta side, and using data from a test report dated April 1989. A piecewise linear model was used to represent transformer saturation based on exciting current tests at $100 \%$ and $110 \%$ rated voltage. For comparison purposes the $500 \mathrm{kV}$ line was represented by two different EMTP line models: an equivalent PI model and constant-frequency, distributed-parameter, traveling-wave model. Line parameters were evaluated at different frequencies using the EMTP Line Constants Program for a typical $500 \mathrm{kV}$ flat conductor layout with two shield conductors. The New Freedom bus was represented by an ideal three-phase voltage source. The simulation was performed under load flow conditions of $1000 \mathrm{MW}$ delivered to New Freedom at unity power factor, which required approximately 370 MVAR from the Salem 1 unit. The geomagnetic disturbance was modeled by a $1 \mathrm{kV}$ dc voltage source connected from ground to the transformer neutral. This produced an average de neutral current of approximately $110 \mathrm{~A}$.

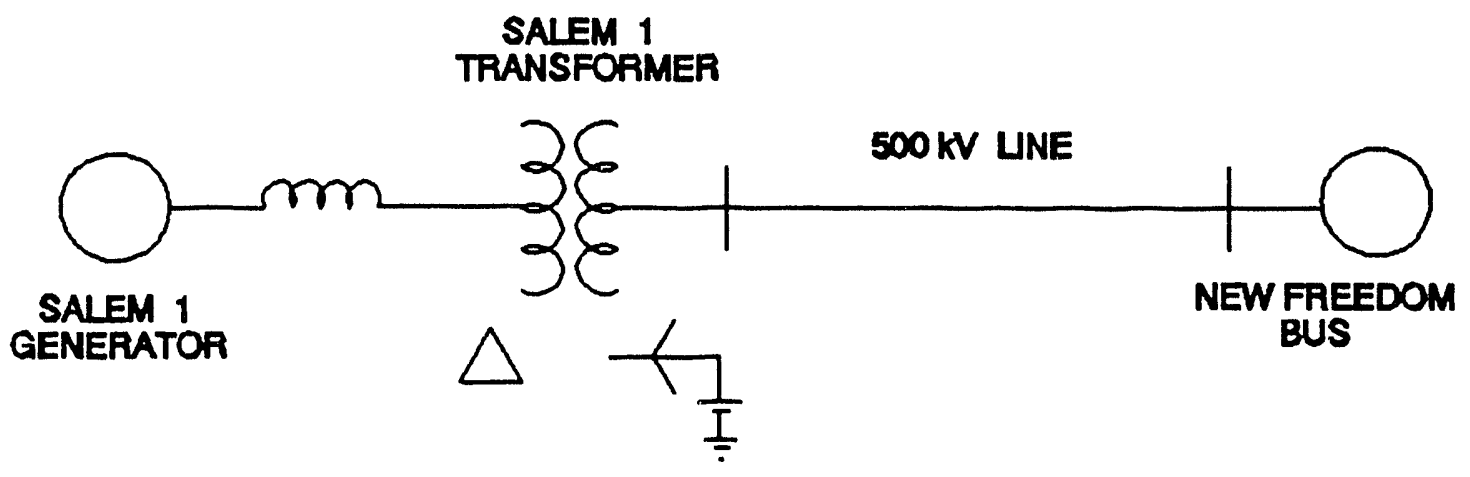

Fig. 4-1. System model for Salem 1 GIC simulation.

Sample waveforms taken from the EMTP simulation are shown in Appendix D, along with a listing of the data input files. The first group of waveforms, Figs. D-1 to D-4, were taken with the transmission line represented by an equivalent PI model, with parameters evaluat $d$ at $60 \mathrm{~Hz}$. This case is similar to an earlier simulation study made on the Salem 1 system. This case asso produced a worstcase scenario for harmonics, although not as severe as predicted in the Salem study. Figure D-1 shows the transformer magnetizing current in phase A, which is similar but slightly larger than in the other two phases. The unbalance is primarily due to the assumed untransposed line data. Figure D-1 shows that 
the magnetizing current is nearly fully offset by the dc neutral current in accordance with the assumed 2 -segment saturation characteristic. The Salem simulation showed much greater saturation, considering that the excitation branch was modeled on the high-voltage side of the transformer, therefore it probably used a multi-segment saturation characteristic (with progressively reduced slopes) that produced correspondingly higher currents. This seems to be a reasonable assumption, although test data was not available to support it.

Figure D-2 shows the waveshape of the transformer neutral current induced by the geomagnetic disturbance (GIC). The harmonics caused by transformer saturation are readily apparent. Figures D-3 and D-4 show the three-phase voltages on both sides of the transformer. Again the harmonic distortion in these waves is readily observed. Although these harmonics are significant, they are not as great as predicted in the Salem study, where higher saturation was observed.

Figures D-5 to D-8 show results taken with a very low frequency $(0.01 \mathrm{~Hz})$, equivalent PI line model. The main object here was to illustrate the importance of correctly representing the low frequency resistance of the line, since typical GIC disturbances change very slowly. In the simulation it was necessary to reduce the geomagnetic surface potential to maintain an average transformer neutral current of about $110 \mathrm{~A}$. Therefore, as shown in Figs. D-5 and D-6, the transformer magnetizing current and neutral current waves look quite similar to Figs. D-1 and D-2. However, Figures D-7 and D-8 show that harmonic levels on the generator and transformer voltages have increased for this case. This is probably due to the reduced damping available at higher frequencies for this low frequency transmission line model.

Figures D-9 and D-10 show the effect of a larger GIC disturbance, where the average transformer neutral current is assumed to be approximately $200 \mathrm{~A}$. Figure D-9 shows the corresponding increase in peak magnetizing current, and Fig. D-10 shows the resulting increase in harmonics in the generator voltages. This simulation was performed using an equivalent PI transmission line model, with parameters evaluated at $60 \mathrm{~Hz}$. As in previous cases, a more realistic saturation characteristic would have most likely produced higher magnetizing currents, with correspondingly higher harmonic levels in the system.

Figures D-11 and D-12 show results taken with a constant frequency $(60 \mathrm{~Hz})$, distributedparameter line model. The conductor data and layout was identical to that used for the equivalent PI model used in the previous figures. The power-frequency equivalence of the two types of line models is evident by observing nearly identical load flows in the simulations, and by nearly identical saturation currents. However, as shown in Figs. D-11 and D-12, the voltage harmonics produced with the distributed-parameter line model are somewhat lower than with the equivalent PI representation. Considering the length of this line $(50 \mathrm{mi})$, the $60 \mathrm{~Hz}$ distributed line model is probably more realistic than a single $60 \mathrm{~Hz}$ equivalent PI model, although its very low frequency characteristics are still not accurate for GIC disturbances. However, the distributed-line approach does allow accurate modeling over a range of frequencies, using a frequency-dependent line parameter representation such as the Jmarti Setup available in the EMTP Line Constants Program and the corresponding time-step simulation model. This would avoid the false resonances possible in a single-frequency equivalent PI model, while providing correct low frequency resistance for the geomagnetic effects. At the same time this approach would provide the correct $60 \mathrm{~Hz}$ impedance for the proper load flow, and the correct high frequency response to generated harmonics. In any case, comparing the results in Appendix D for the various line models, shows the importance of using a good representation for the transmission network. This is probably just as important as modeling the transformer saturation characteristic. 


\subsubsection{Plant Description}

Detailed Simulation of the entire electrical distribution systems inside the plant of another nuclear power generating station, other than Salem, is considered for this study. The plant electrical power system consists of the unit generator, the switchyard, and the unit auxiliary power distribution system. The unit generator is connected directly to the generator step-up transformer and the normal station service transformer through isolated phase buses. The generator step-up transformer steps up the output of the unit generator from $25 \mathrm{kV}$ to a nominal $345-\mathrm{kV}$ transmission system voltage. The normal station service transformer steps down the unit generator voltage from $25 \mathrm{kV}$ to $13.8 \mathrm{kV}$ and provides an onsite (ac) power source to the unit auxiliary power distribution system.

The switchyard has two separate and independent sections: the $345-\mathrm{kV}$ switchyard and the $115-\mathrm{kV}$ switchyard. The output of the generator step-up transformer is connected to the 345-kV switchyard which connects the unit generator to the outgoing transmission system. The $115-\mathrm{kV}$ switchyard receives power from two separate offsite power sources through two physically and electrically independent incoming circuits. The two circuits feed two separate reserve station service transformers and an auxiliary boiler transformer. The reserve station service transformers step down the offsite power from $115 \mathrm{kV}$ to 13.8 $\mathrm{kV}$ and $4.16 \mathrm{kV}$, and provide two independent offsite power sources for the unit auxiliary power distribution system. The auxiliary boiler transformer steps down the offsite power from $115 \mathrm{kV}$ to 13.8 $\mathrm{kV}$ and $4.16 \mathrm{kV}$. Its $13.8 \mathrm{kV}$ winding supplies power to the auxiliary boiler and associated equipment; the $4.16 \mathrm{kV}$ tertiary winding provides a backup source for the emergency $4.16 \mathrm{kV}$ buses.

The unit auxiliary power distribution system feeds all unit auxiliary loads through $13.8 \mathrm{kV}$ switchgear, $4.16 \mathrm{kV}$ switchgear, $600 \mathrm{~V}$ load centers, $600 \mathrm{~V}$ motor control centers, and various ac and dc distribution panels. The system is divided into nuclear nonsafety-related and nuclear safety-related systems. The nuclear nonsafety-related auxiliary power distribution system feeds all non-Class $1 \mathrm{E}$ unit auxiliary loads. Under normal plant operating conditions, it is energized from the normal station service transformer. During startup and normal shutdown conditions, it is energized from offsite power sources through reserve station service transformers. A normal $125 \mathrm{~V} \mathrm{dc}$ system, consisting of batteries, battery chargers, and distribution panels, provides a reliable source of power for protection, control, and instrumentation loads and dc motors under normal and emergency conditions of the plant. $\mathrm{A} \pm 24 \mathrm{~V} \mathrm{dc}$ system provides a reliable source for the neutron monitoring system.

The nuclear safety-related auxiliary power distribution system supplies all Class 1E unit auxiliary loads. This system is divided into three independent divisions. Division $I$ and Division II are independent redundant divisions and supply all nuclear safety-related auxiliary loads except the high pressure core supply (HPCS) system. The HPCS system and related equipment are supplied by Division III. All three divisions are normally energized from the offsite power sources through reserve station service transformers. The auxiliary boiler transformer can be connected manually to act as a backup source for either the Division I or Division II supply.

Each of the three divisions of the nuclear safety-related auxiliary power distribution systems has its own independent standby diesel generator. In the event of a LOCA and/or loss of offsite power, each division is energized from its own standby diesel generator. A $125 \mathrm{~V}$ emergency dc power system feeds all safety-related dc protection, control, and instrumention loads and safety-related dc motors under normal operation of the plant as well as during emergency conditions. The system is divided into three independent divisions each consisting of its own battery, primary and backup battery chargers, switchgear, 
motor control centers, and distribution panels. Each division feeds the dc loads associated with the corresponding divisions of the nuclear safety-related auxiliary power distribution system.

Figure 4-2 is a simplified one-line diagram of the overall plant electrical distribution system. It shows the $25 \mathrm{kV}$ generator bus, which connects to the $345 \mathrm{kV}$ transmission system through the unit stepup transformer, and also connects to the two $13.8 \mathrm{kV}$ station service buses through the three-winding normal station service transformer. It also shows the two reserve station service transformers fed from independent $115 \mathrm{kV}$ offsite circuits A and B. These transformers normally supply the emergency loads through the $4.16 \mathrm{kV}$ windings, as indicated by the normally-closed breaker connections to emergency buses ENS-1,2,3. The $13.8 \mathrm{kV}$ windings are available to supply the non-emergency distribution system for loss of on-site power. Offsite $115 \mathrm{kV}$ circuit A also supplies power to the auxiliary boiler transformer. Each $13.8 \mathrm{kV}$ bus normally feeds two or three $4.16 \mathrm{kV}$ nonsafety-related buses, as shown symbolically by the four equivalent NNS buses. Each $13.8 \mathrm{kV}$ bus also fans out into several $600 \mathrm{~V}$ buses, which feed various motors, distribution panels and other loads. Some $600 \mathrm{~V}$ buses, in turn, also feed several lower voltage points, such as three-phase $208 \mathrm{~V}$ and $240 / 120 \mathrm{~V}$ single-phase loads. The numerous $600 \mathrm{~V}$ and lower voltage buses fed from the $13.8 \mathrm{kV}$ buses are represented symbolically in Fig. 4-2 by the two outside pairs of buses. Each $13.8 \mathrm{kV}$ bus also supplies several large motors, shown symbolically as an equivalent motor at each bus, plus two specific pump motors that are interconnected by a breaker arrangement between the buses. The $4.16 \mathrm{kV}$ nonsafety-related buses also supply several motor loads, shown symbolically as a single motor per bus, although two of these buses can also supply the emergency system under certain conditions. The nonsafety-related $4.16 \mathrm{kV}$ buses also fan out into several $600 \mathrm{~V}$ buses, which in turn feed numerous lower voltage ac and dc loads. The $125 \mathrm{~V}$ dc system interfaces with the low voltage ac system through various battery chargers and UPS units. Again this lower voltage system is represented in Fig. 4-2 by only a few equivalent buses.

Figure 4-3 is a simplified one-line diagram of the safety-related, ernergency distribution system. As mentioned earlier, this system is partitioned into three separate divisions (I,II and III). Division I is normally supplied from the $4.16 \mathrm{kV}$ winding of the reserve station service transformer connected to 115 $\mathrm{kV}$ circuit $\mathrm{A}$, and division II from the $4.16 \mathrm{kV}$ winding of the reserve station service transformer connected to $115 \mathrm{kV}$ circuit B. The $4.16 \mathrm{kV}$ buses in all divisions fan out into several $600 \mathrm{~V}$ and lower voltage buses, which in turn feed various motor loads, distribution panels, etc.. Again this is shown symbolically in the diagram by only a few buses and typical load elements.

Figure 4-4 is simplified one-line diagram of the offsite power transmission system connected to the plant. It consists of a single major $345 \mathrm{kV}$ tie-line connecting the plant to the primary $345 \mathrm{kV}$ remote substation. The offsite $115 \mathrm{kV}$ power is assumed to be supplied by the local $345 \mathrm{kV}$ switchyard through separate step-down transformers. The main GIC path is therefore through the generator step-up transformer high-voltage grounded neutral point, through the $345 \mathrm{kV}$ line and the primary $345 \mathrm{kV}$ remote bus. It may also be possible for some level of GIC to pass through the grounded neutrals of the reserve station service transformers and back through the $345 \mathrm{kV}$ system. The primary $345 \mathrm{kV}$ remote bus is also connected to two secondary $345 \mathrm{kV}$ remote buses, as shown in the diagram. The actual transmission network would normally be more complicated than Fig. 4-4 shows. However, for purposes of this investigation, this network equivalent appears to be an adequate representation.

\subsubsection{EMTP Simulation Model}

Figure 4-5 is a one-line diagram of the EMTP simulation model of a typical power plant and its offsite transmission system. The simulation is actually a fully detailed three-phase representation of the system. The generator is represented by either a simple voltage-behind-subtransient-reactance, as shown 


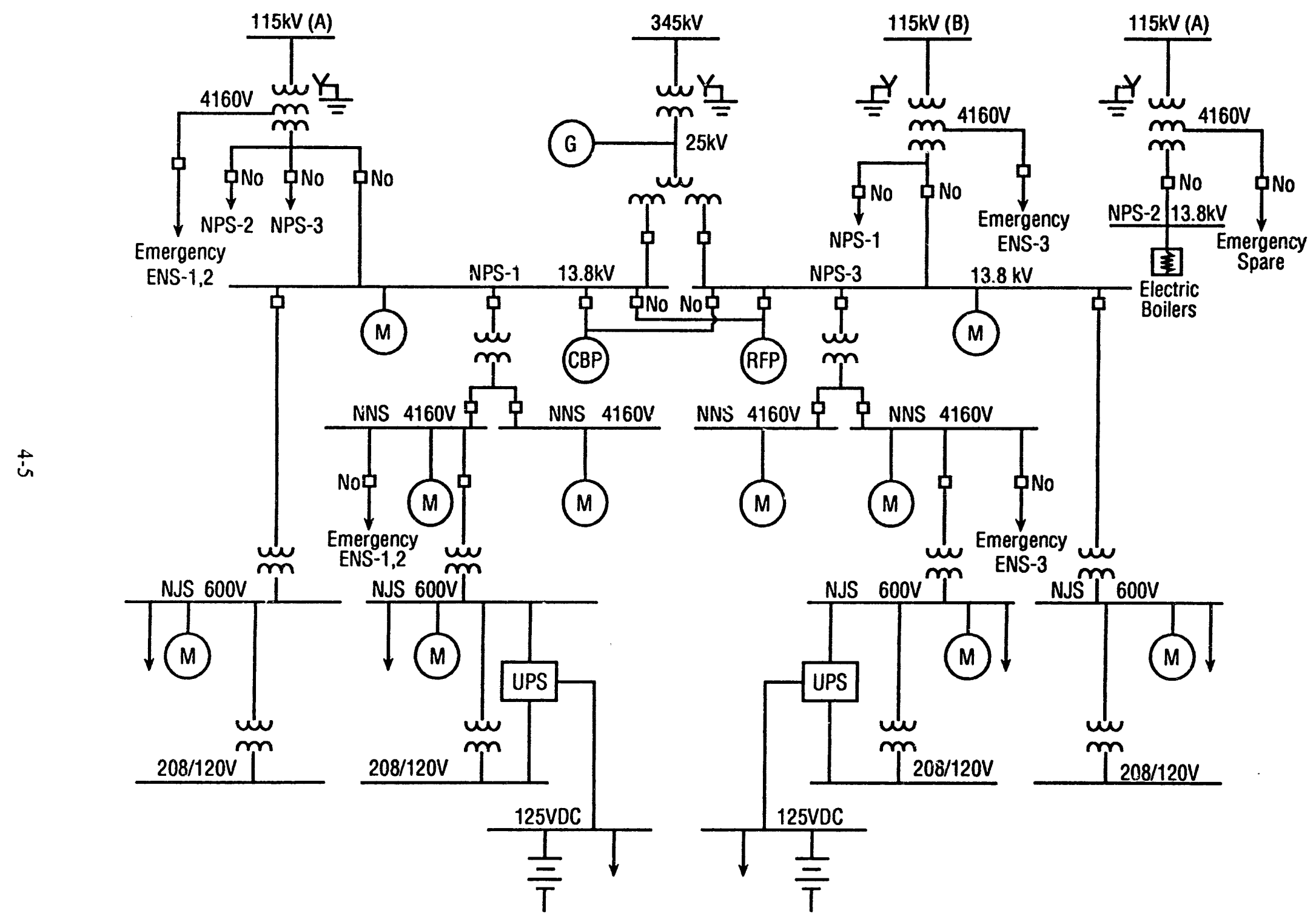

Fig. 4-2. Overall plant distribution. 


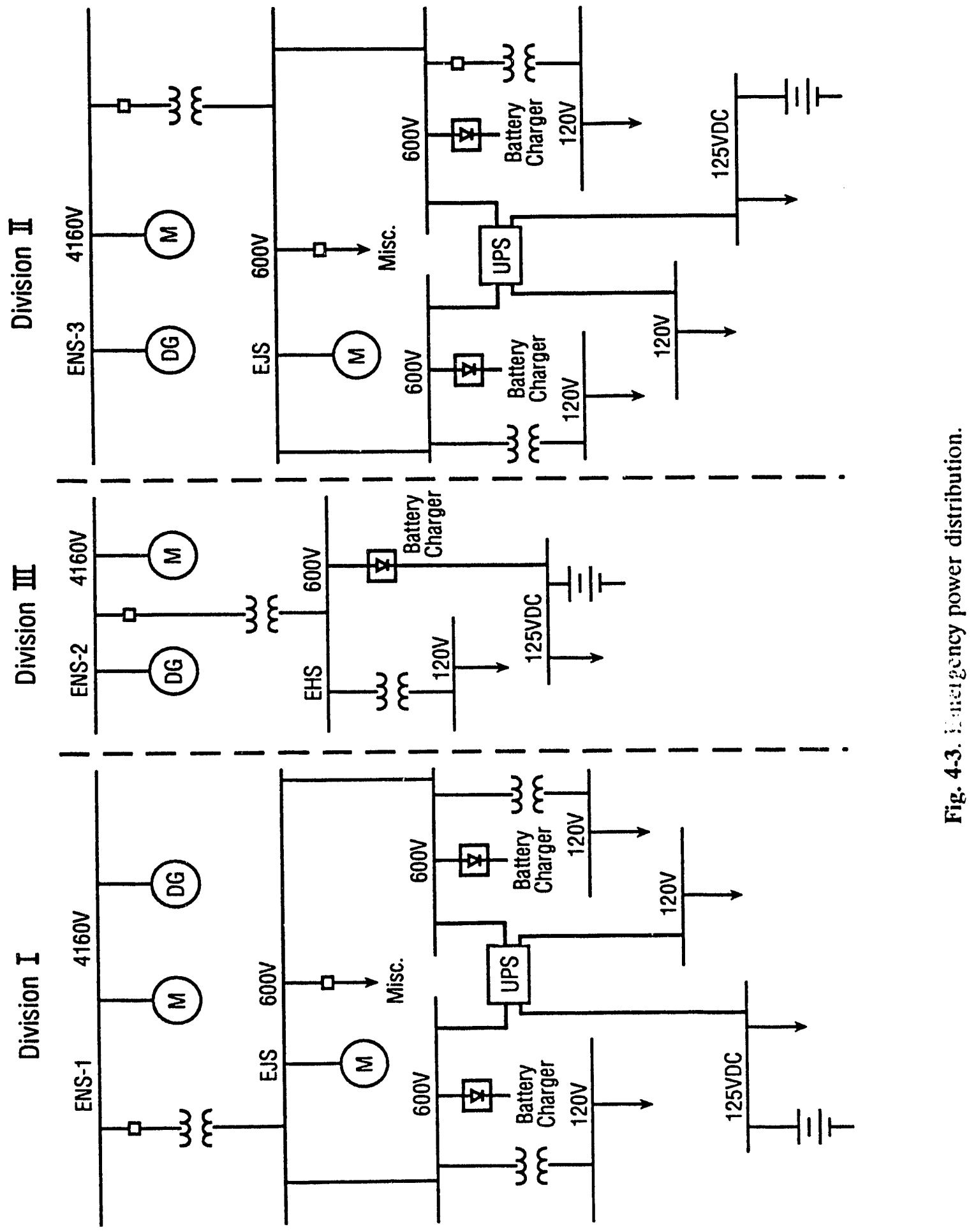




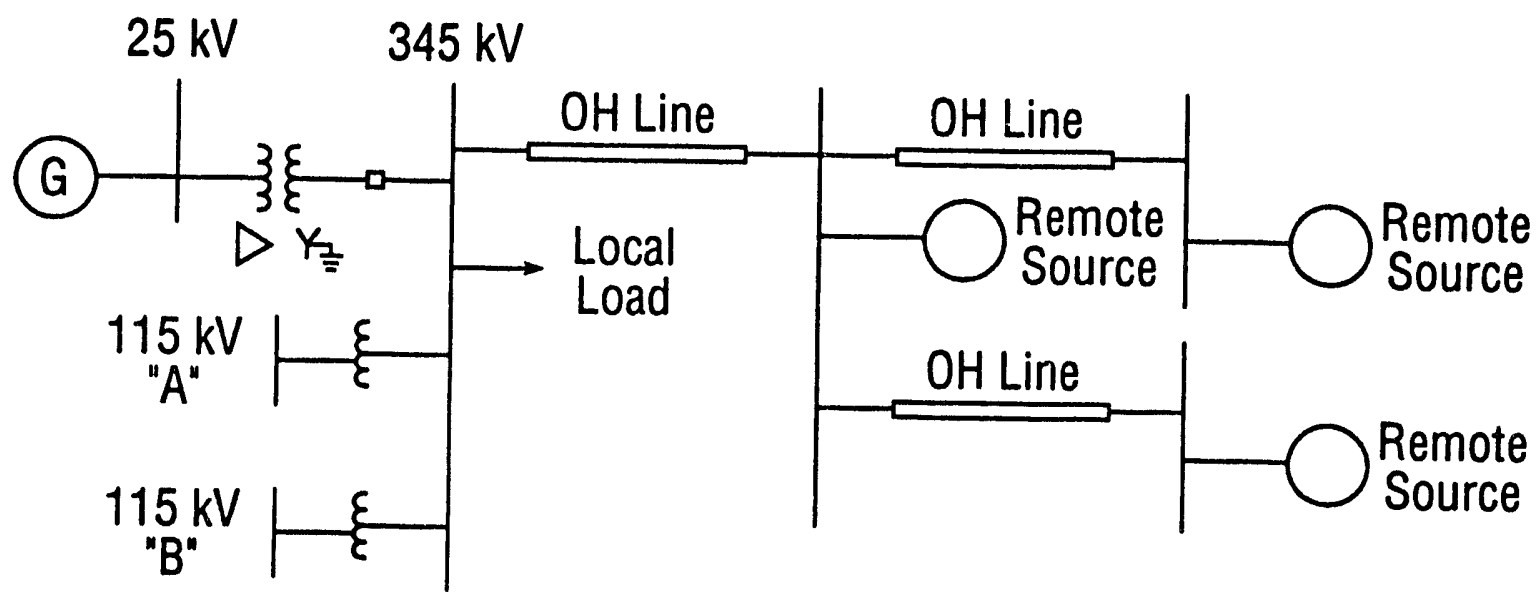

Fig. 4-4. Offsite power system.

studies in the diagram, or it can be represented by a more detailed d-q axis model (Parks equations) with mechanical system and exciter included. Preliminary comparisons between these two approaches showed very little difference in results for problems involving GIC-induced harmonics. Therefore, the simpler generator model is preferred, since it requires much less computer time to implement. If later transient studies require the more detailed generator model, it is ready and available for immediate use. The deltagrounded-wye generator step-up transformer (T1) is modeled in full detail, with core loss, winding loss, leakage inductance, and magnetic saturation all included. The step-up transformer is connected to the transmission system, represented by a single overhead line model connected to an infinite bus. It can easily be expanded later to include more transmission lines if necessary. The overhead line can be modeled in various forms: equivalent PI section(s), a constant-frequency distributed-parameter model, or a frequency-dependent distributed-parameter model. The advantages and disadvantages of these line model options were discussed briefly in section 4.1. The length of the line, which could have an important influence on system behavior, may also be easily varied in each of the options.

As Fig. 4-5 shows, this simulation attempts to model the essential features of both the nonsafetyrelated and safety-related systems, without over-complicating the picture. It is important initially to keep things as simple as possible until the basic mechanisms are better understood. This is especially important in using a transients program like EMTP, in order to minimize the computer time spent for each run. Many computer runs are necessary to understand the problem and to acquire enough simulation data for a meaningful evaluation.

Figure 4-5 shows the system configuration for normal operation, where the normal station service transformer (T2) feeds the nonsafety-related network and the reserve station service transformer (T3) feeds the safety-related, emergency system. The system configuration can easily be changed for other modes of operation by changing the status of the various switches available in the simulation, or by 


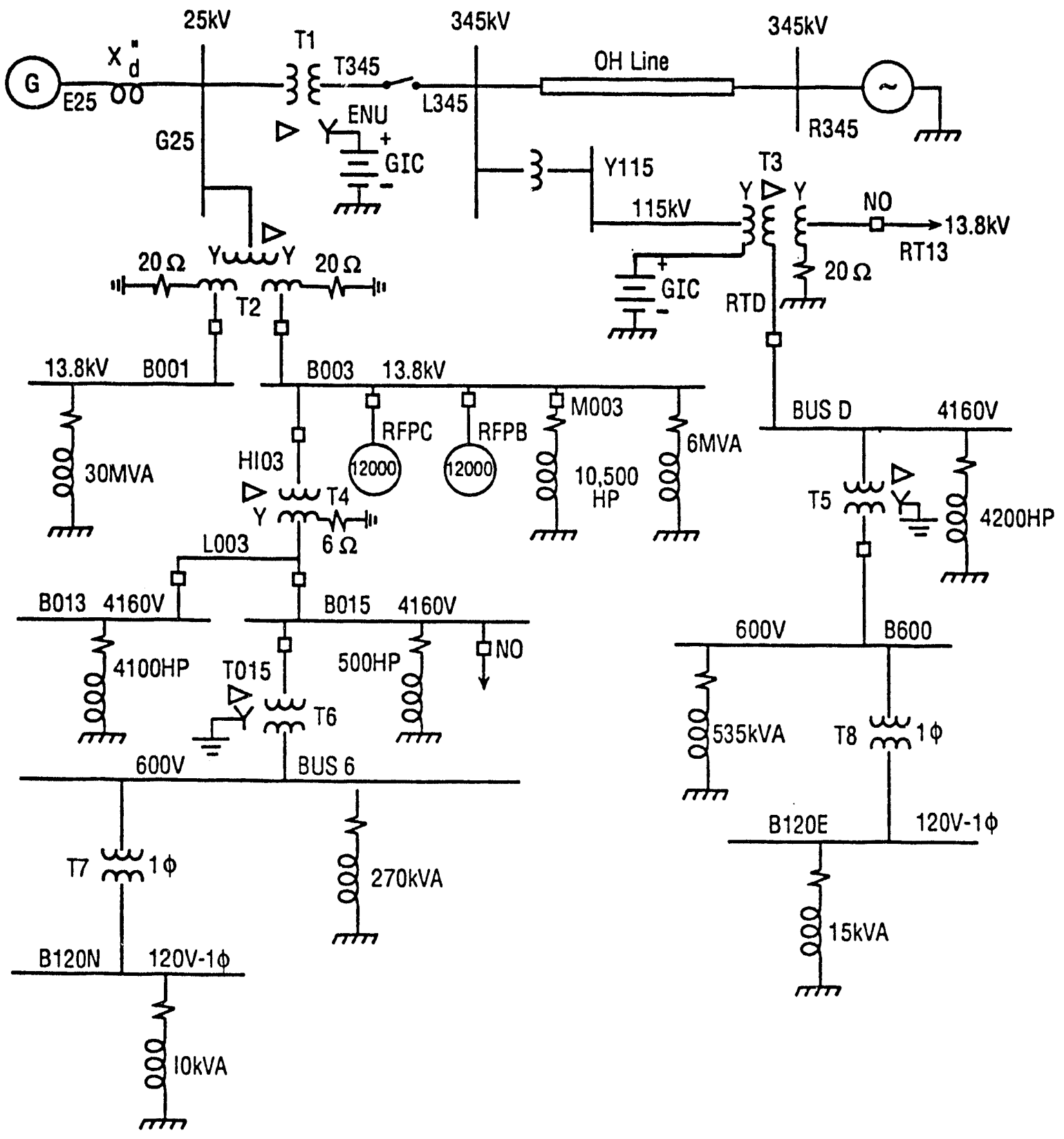

Fig. 4-5. Simulation model. 
manually changing node names in the EMTP data input file. Both the normal and the reserve station service transformers are represented in full three-phase, three-winding detail, with losses, leakage reactances, and magnetic saturation included. Because of symmetry it is necessary to model in detail only one safety division in the emergency system, and only one of the two $13.8 \mathrm{kV}$ nonsafety-related buses. Note that, although the two systems are not coupled in the plant, there is potential coupling at the transmission level in the offsite power system. The coupling may be important in GIC studies, therefore, it is necessary in this mode of operation to simultaneously represent the nonsafety-related and the safetyrelated systems together in the simulation.

For the nonsafety-related system, the normal station service transformer (T2) feeds $13.8 \mathrm{kV}$ buses B001 and B003. Bus B003 supplies two 12,000 Hp pump motors, a static equivalent of several other motors, and a static equivalent of several $600 \mathrm{~V}$ loads connected through $13.8 \mathrm{kV} / 600 \mathrm{~V}$ delta-wye transformers. The $12,000 \mathrm{Hp}$ motors are modeled in full transient detail as three-phase rotating machines, including losses, magnetizing current, and rotor/load inertia. Bus B003 also feeds two $4.16 \mathrm{kV}$ buses, $\mathrm{B} 013$ and $\mathrm{B} 015$, through a delta-wye transformer (T4). These buses are loaded with static equivalents of several additional motors, and B015 also feeds a $600 \mathrm{~V}$ bus, called BUS6 in the EMTP data file, through another delta-wye transformer (T6). BUS6 in turn supplies a $270 \mathrm{kVA}$ static equivalent load, and feeds a 120/240 V bus, B120N, through a single-phase transformer (T7). This particular 120/240 V bus supplies power to fire protection panels and miscellaneous control circuits.

For the emergency system, the reserve station service transformer (T3) $4.16 \mathrm{kV}$ winding supplies the division II main bus, BUSD. BUSD in turn feeds several $600 \mathrm{~V}$ buses and distribution panels, which are represented by the equivalent bus $B 600$ fed by delta-wye transformer (T5). B600 feeds a static equivalent $535 \mathrm{kVA}$ load, and supplies a $120 \mathrm{~V}$ bus, B120E, through a $25 \mathrm{kVA}$ single-phase transformer (T8). B120E is an emergency relay supply panel.

The first important objective of the simulation model shown in Fig. 4-5 is to give a preliminary evaluation of GIC-induced harmonic magnitudes at various levels and locations in the system, from the station service transformers down to the low voltage control buses. In order to minimize initial model complexity, careful attention was given to bus selection, model parameters and system configuration. Later work will focus on more detailed models, particularly in the critical lower voltage areas, in order to investigate the effect of harmonics on the safety-related control and protective functions. Another objective of this and future simulations is to investigate the compounding effects of normal station transients superimposed on GIC disturbances. This simulation model is adequate for some studies of this type, but will again require further detail, particularly in the lower voltage control-related areas.

Model data for the simulation in Fig. 4-5 is summarized in Appendix E. Input data required by EMTP was not completely available for an actual plant. For many of the components modeled in the simulation diagram, this would have required a close working cooperation with a utility company that currently operates a nuclear plant. In place of this, typical parameters for each component in the system were derived from published documents such as Plant Final Safety Analysis Reports, power engineering handbooks [12,13], and other published data available in the literature. This was a very time-consuming process, but eventually resulted in a fairly realistic set of system data. Any remaining uncertainty in data can be mitigated by making multiple studies over a reasonable range of the uncertain parameter. Appendix E shows assumed and derived data for the key system components, including the $345 \mathrm{kV}$ overhead line, generator reactance, transformer parameters and saturation characteristics, motor parameters and load data. Also listed in Appendix E are the EMTP data input files used in the simulation. 


\subsubsection{Simulation Study Results}

The first phase of this study involved the steady-state simulation of the model shown in Fig. 4-5 using the nominal system data listed in Appendix E. In the results that follow, this mode of operation is referred to as "normal operation" for the system. As stated earlier, in this mode the normal station service transformer feeds all nonsafety-related loads, and the reserve station service transformers feed the safety-related (emergency) loads. It is important to note that in this mode of operation, the normal station loads on the nonsafety-related buses are much greater than the loads on the safety-related emergency buses and in fact the emergency system is very lightly loaded. As later results will show, this fact makes the emergency system buses in this mode more susceptible to large harmonic voltage distortion than the nonsafety-related buses. This is primarily due to its relatively high impedance to current flow, including harmonic currents.

\subsubsection{Effect of GIC current levels}

For convenience all waveform recordings taken from the simulation are contained in Appendix $\mathrm{F}$ for the various cases studied. Figures F-1 through F-10 show sample recordings of steady-state operation at various voltage levels, in the normal system mode. This first group of recordings was taken for a total GIC of approximately $100 \mathrm{~A}$. Most of this DC current flows through the HV neutral of the generator step-up transformer, although a portion of it also flows through the HV neutral of the reserve station service transformer. This is a consequence of the coupling of the system at the offsite transmission level, where the local $345 \mathrm{kV}$ substation interconnects the $345 \mathrm{kV}$ system and the $115 \mathrm{kV}$ system. Figure F-1 shows the three-phase magnetizing currents in the generator step-up transformer. In the simulation these are referred to the $24 \mathrm{kV}$ delta winding on the generator. The peak saturation currents are well over $2000 \mathrm{~A}$, with phase $\mathrm{A}$ being the largest, due to a slightly unbalanced system. This compares to a normal unsaturated magnetizing peak of about $137 \mathrm{~A}$. Figure F-3 shows the actual neutral current (upper trace) flowing in the step-up transformer, having an average value of approximately $100 \mathrm{~A}$ on the HV side. This value is related to the average value of the saturated current wave in Fig. F-1 by the winding turns ratio of the transformer, which in this case is $n_{1}: n_{2}=345 / \sqrt{ } 3: 24$. If the saturated current pulse is approximated by a half sine wave, a rough approximation for the peak magnetizing current is given by

$$
I_{p k}=\frac{\pi}{2} \frac{n_{1}}{n_{2}} I_{\text {news }}
$$

where $I_{p k}$ is the peak magnetizing current and $I_{\text {neut }}$ is the average neutral (GIC) current. Equation (4-1) is very conservative for narrow saturation waves like shown in Fig. F-1, since this wave has a considerably smaller area than a half sine wave.

Figure F-2 shows the three-phase magnetizing currents in the reserve station service transformer. Although a small proportion of the total GIC also flows in the HV grounded neutral of this transformer, the nagnetizing currents seem unexpectedly high. However, this is explained approximately by equation (4-1) realizing that the magnetizing current is referred to the $4.16 \mathrm{kV}$ winding, which makes the effective winding turns ratio about six times that of the generator step-up transformer. This is another reason that in the normal operating mode, the emergency system, which is fed by the reserve station service transformer, may be more sensitive to GIC-induced harmonics. The peak saturation current in this transformer is over $1500 \mathrm{~A}$, compared to the normal unsaturated peak current of about $142 \mathrm{~A}$. 
For the normal mode of operation the saturation current waves in Figs. F-1 and F-2 represent system input sources that produce harmonics in the transmission line and also in the station distribution network. Although not always the case, the harmonic levels produced are generally proportional to the magnitude of the transformer saturation currents. The magnitude of these saturation currents depend on the average neutral current, which in turn, depends mainly on the offsite line and ground resistances and the ESP (earth surface potential) produced by the geomagnetic disturbance. On the other hand, the resulting harmonic current flow depends on the impedances of both the onsite and the offsite systems. As shown in Fig. 4-6, the saturated transformer can be thought of as a current source of harmonics that feeds two parallel branches, representing the onsite and offsite systems. It is clear from this simple model that the distribution of harmonics in the station will depend on many factors, including the characteristics of the transmission network. For example, a low line inductance could result in the majority of induced harmonics being bled off into the transmission system, whereas, a resonant condition could have the opposite effect at one particular frequency.

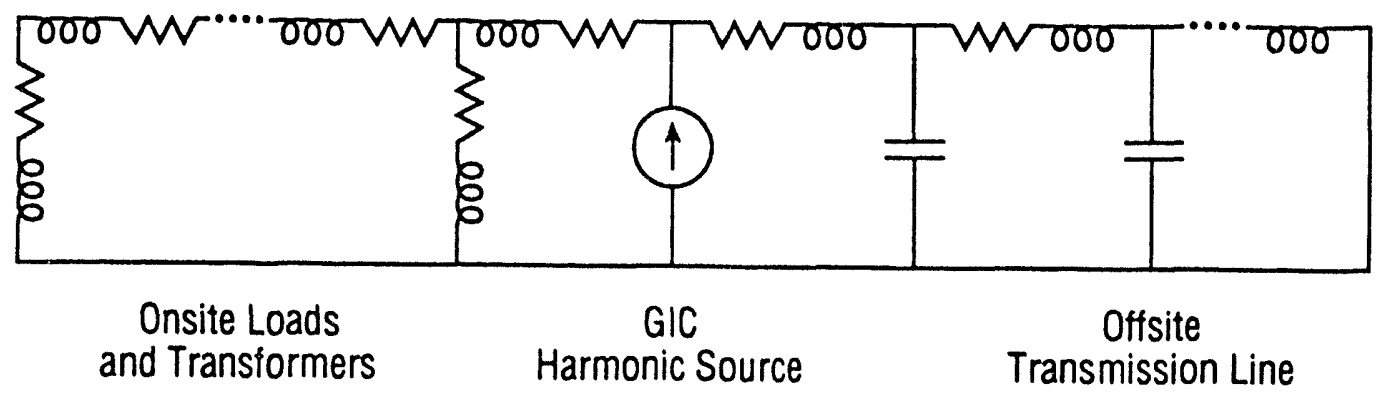

Fig. 4-6. Simple model for distribution of GIC-induced harmonics between power station and transmission system.

Another major factor in determining station voltage harmonics is the station loading. Light station loading implies a high impedance to both fundamental and harmonic currents, which in turn means relatively low harmonic voltage drops through the various transformers leading to the sensitive low voltage circuits. Therefore light loading conditions may produce the most severe harmonics at the lower voltage levels. Of course, the original source of these harmonics is the nonlinear saturation current, which in itself is not a fixed quantity. For example, as the saturation level increases, the magnetizing current pulses change shape, typically becoming broader at the base, appearing more like a half sine wave. This has some effect on the frequency spectrum, and therefore, the frequencies of harmonics in the station will also vary with saturation level.

Figurcs 4-7 and 4-8 show the frequency spectrums of the magnetizing currents in the generator step-up transformer and the reserve station service transformer. The two transformers produced quite similar looking spectrums, except for some differences in magnitudes of individual harmonics. In addition to the normal odd harmonics produced by symmetrical saturation, there are some very strong low order even harmonics that can be particularly troublesome. Although the triplen harmonics will be effectively blocked by the delta windings, the other strong lower order harmonics could propagate into the station circuits. 


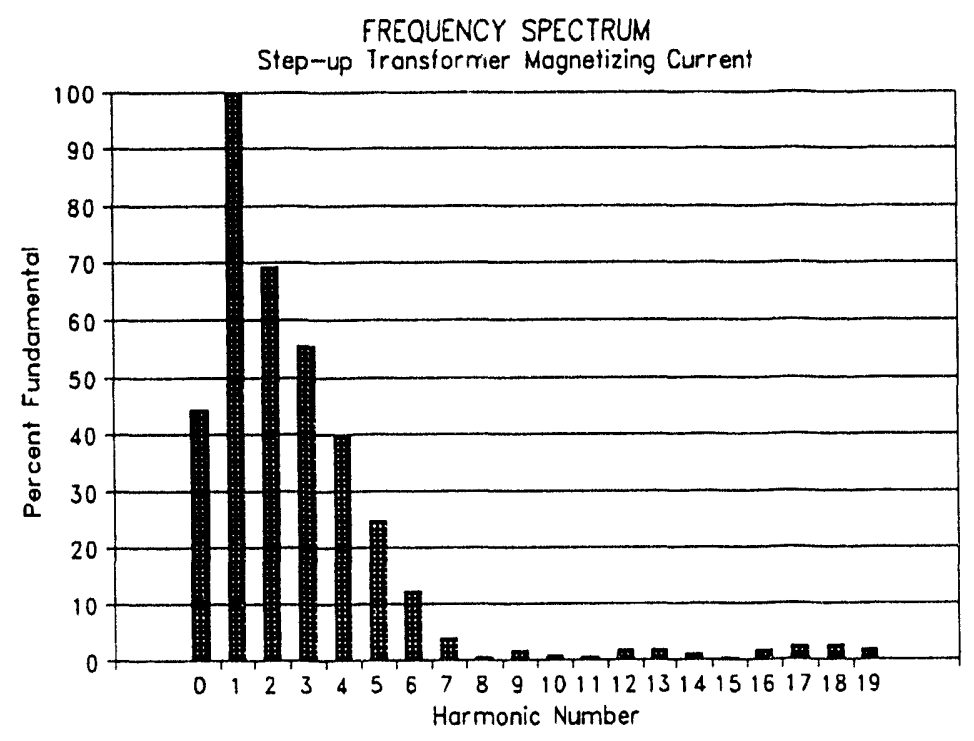

Fig. 4-7. Frequency spectrum of generator step-up transformer magnetizing current.

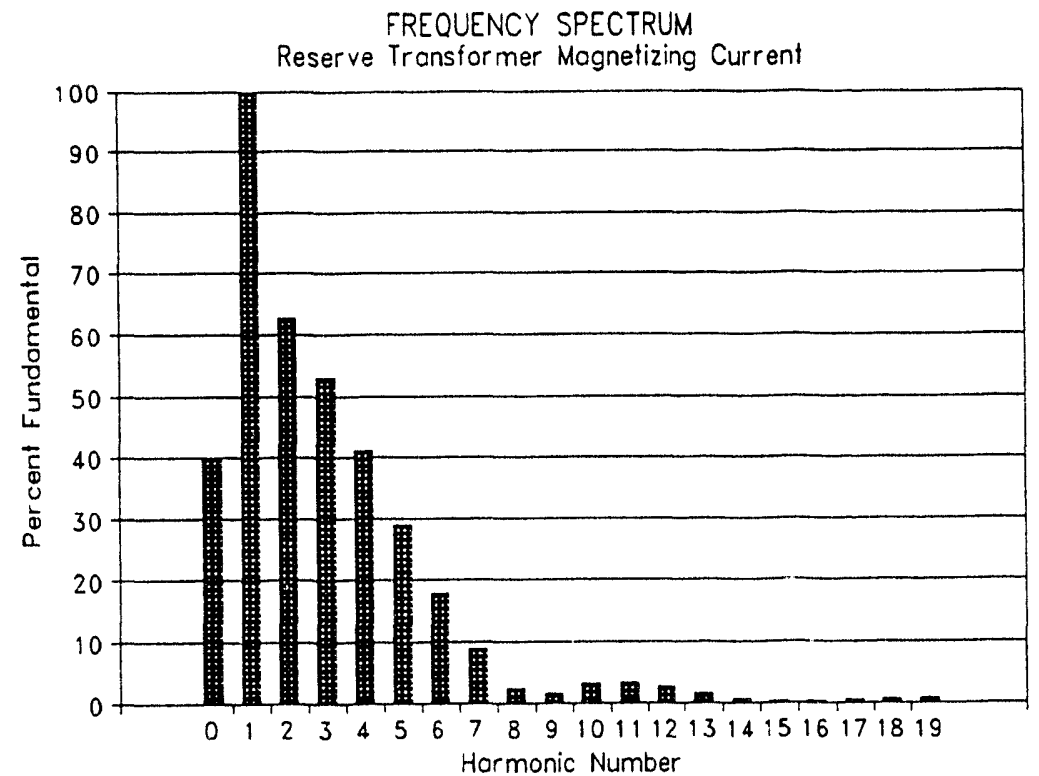

Fig. 4-8. Trequency spectrum of reserve station service transformer magnetizing current. 
Figures F-4 through F-10 show the steady-state voltage waves at the various levels in the plant. Figure F-4 shows the $345 \mathrm{kV}$ transmission level voltages on the high side of the step-up transformer. Harmonic distortion is clearly evident on these waves, as quantified by the frequency spectrum shown in Fig. 4-9 for the phase A voltage. Most of the harmonics are small (less than 1\%) with the dominant harmonic the $19^{\text {th }}$ at $3 \%$. The THD (total harmonic distortion) is $3.44 \%$. Figure F-5 shows the generator voltages (on the delta side of the step-up transformer). Harmonic distortion is again visibly evident on these waves, but does not appear as severe as on the transmission level. Figure 4-10 shows the frequency spectrum for phase A generator voltage. The effect of the delta winding is clearly evident, as all triplen harmonics have disappeared in the generator voltage, which accounts for its cleaner appearance and lower THD of $2.16 \%$. Figure F-7 shows the $4.16 \mathrm{kV}$ voltages on the LV winding of the reserve station service transformer. These appear more distorted than on either side of the step-up transformer, which is confirmed by the frequency spectrum shown in Fig. 4-11 for the phase A voltage. This shows that the triplen harmonics are effectively blocked from entering the station by the delta winding, but several other harmonics have not been attenuated significantly by the transformer, mainly due to the very light loading of this emergency circuit. The THD at this voltage level was measured at 3.97\%. Figures F-9 and F-10 show the voltage waves at the selected $600 \mathrm{~V}$ and $120 \mathrm{~V}$ points in the simulation. The voltages in the nonsafety-related system appear nearly sinusoidal with very little visible distortion, while the emergency system voltages are much more distorted. Again this is due to the significant difference in loading between the two systems, which accounts for the filtering effect of the transformers in the nonsafetyrelated system. Figure 4-12 shows the frequency spectrum for the $120 \mathrm{~V}$ emergency system voltage shown in Fig. F-10. Comparing this with Fig. 4-11 indicates very little filtering between the $4.16 \mathrm{kV}$ and the 120 $V$ level, with all significant harmonics showing very little reduction, and the THD remains over $3 \%$ at the low voltage level.

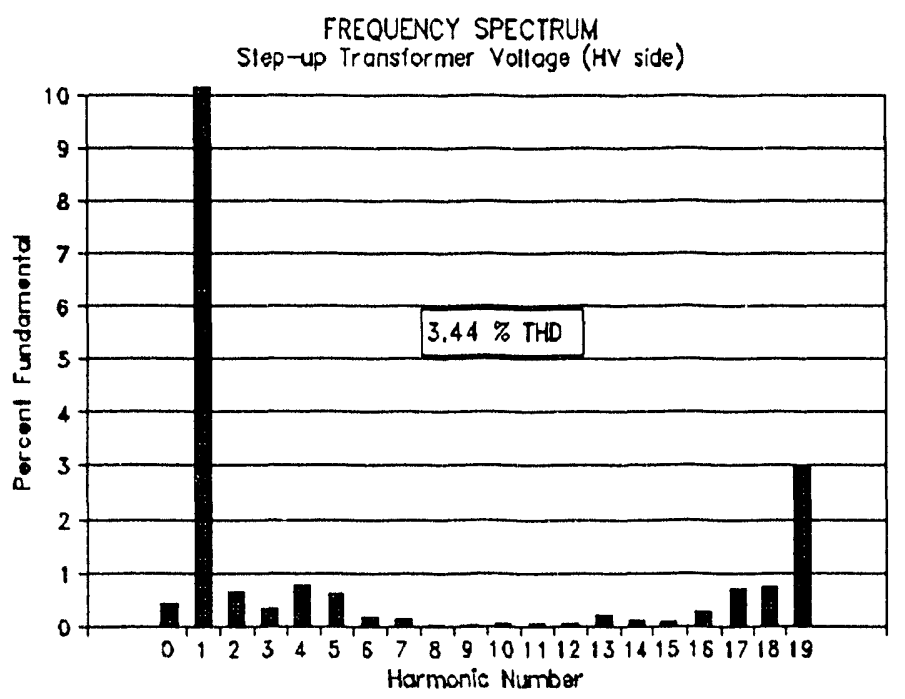

Fig. 4-9. Frequency spectrum of phase A step-up transformer voltage on HV side $(345 \mathrm{kV})$.

In order to explore the effects of higher transformer saturation currents, studies were performed at $200 \mathrm{~A}$ and $500 \mathrm{~A}$ GIC levels. Figures F-11 through F-16 show sample results for a $200 \mathrm{~A}$ GIC. Figures F-11 and F-12 show the steady-state magnetizing currents for the step-up transformer and the reserve 


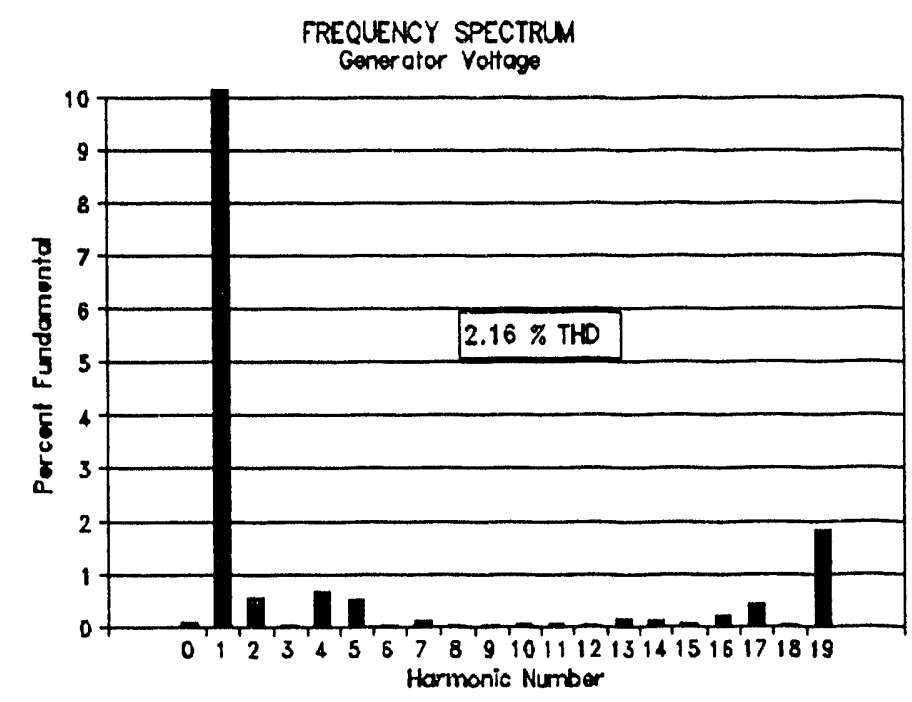

Fig. 4-10. Frequency spectrum of phase A generator voltage.

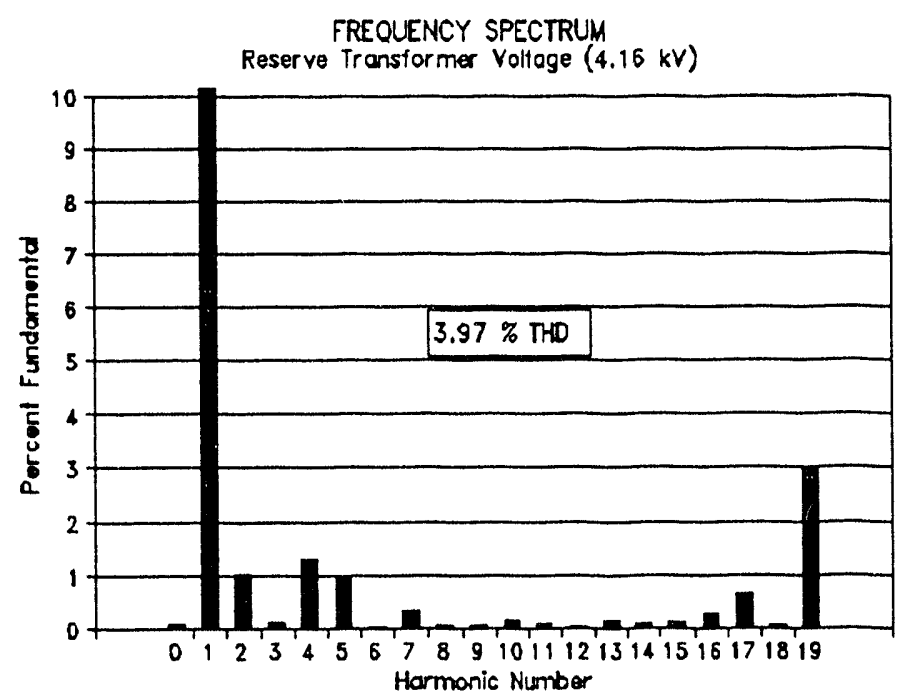

Fig. 4-11. Frequency spectrum of phase A reserve station service transformer voltage on LV side $(4.16 \mathrm{kV})$.

station service transformer for this new condition. As expected, the saturation currents are significantly higher in both transformers, as compared to the results shown in Figs. F-1 and F-2. Figure F-13 shows the generator voltages for the $200 \mathrm{~A}$ GIC, which shows a visibly higher distortion than with the previous 


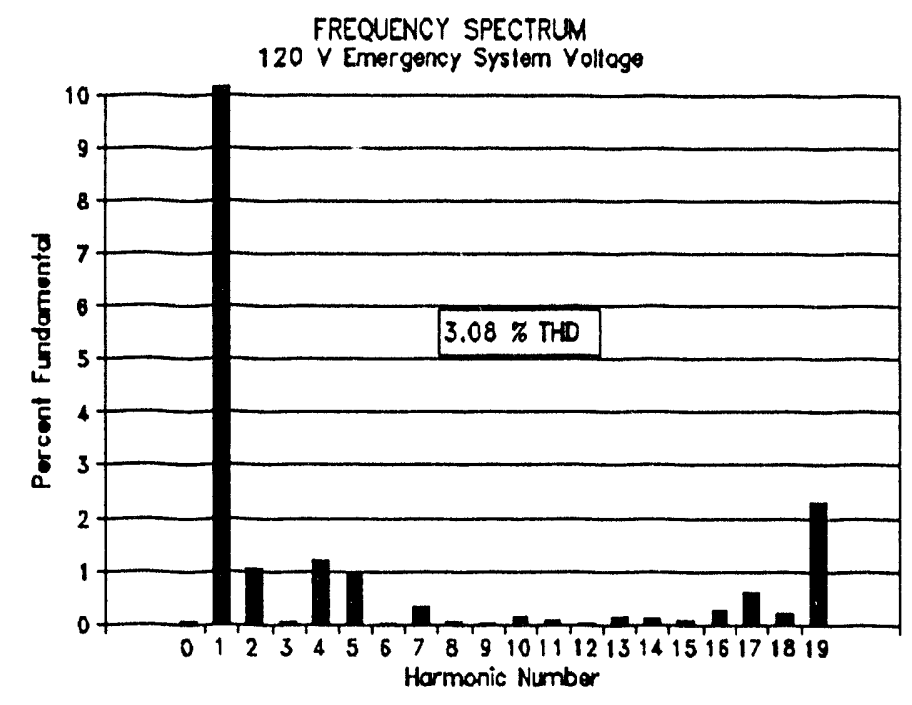

Fig. 4-12. Frequency spectrum of $120 \mathrm{~V}$ emergency system voltage.

case. Figure 4-13 confirms this with noticeably larger harmonic levels and a higher THD of $3.29 \%$. The increase in harmonic levels is even more apparent in the reserve station service transformer voltages, as indicated by Figs. F-14 and 4-14, which show the $4.16 \mathrm{kV}$ waveforms and the frequency spectrum for phase A. Although the delta windings are still effective in blocking the triplen harmonics, several other harmonics are able to pass through the transformer with low attenuation, resulting in a $5.58 \%$ THD at the $4.16 \mathrm{kV}$ bus. Figures F-15 and F-16 show the simulated voltages at the $600 \mathrm{~V}$ and the $120 \mathrm{~V}$ levels for the normal and emergency systems. Again the nonsafety-related buses show lower distortion than the safety-related buses, due to the differences in loading of the two systems. To quantify this, Fig. 4-15 shows the frequency spectrum for the simulated $120 \mathrm{~V}$ bus voltage in the emergency system. Comparing with Fig. 4-14 shows that very little harmonic attenuation occurs between the $4.16 \mathrm{kV}$ level and the 120 $\mathrm{V}$ level, especially for the lower order harmonics. The THD for this $120 \mathrm{~V}$ bus voltage is $4.66 \%$.

Figures F-17 and F-18 show the magnetizing currents in the step-up and reserve station service transformers for the extreme case of $500 \mathrm{~A} \mathrm{GIC}$. Clearly the current saturation levels are very high for this condition, with well over $6000 \mathrm{~A}$ peak current (referred to the $24 \mathrm{kV}$ side) in the step-up transformer. The shape of the magnetizing current pulses has also changed somewhat, compared to the earlier cases of $100 \mathrm{~A}$ and $200 \mathrm{~A}$ GIC, now appearing more like a half sine wave. As shown in Fig. 4-16, this change in shape affects the frequency spectrum of the magnetizing current wave. Comparing with Figs. 4-7 and 4-8, note the general shift in the spectrum toward the lower harmonics as the GIC increases. While the magnitude of the fundamental increases substantially, the percentage of second harmonic increases only slightly, and the percentage of most harmonics above the second are reduced as the GIC increases. This effective reduction in higher order harmonics in the magnetizing currents could also have some effect on the harmonics that propagate into the station circuits. 


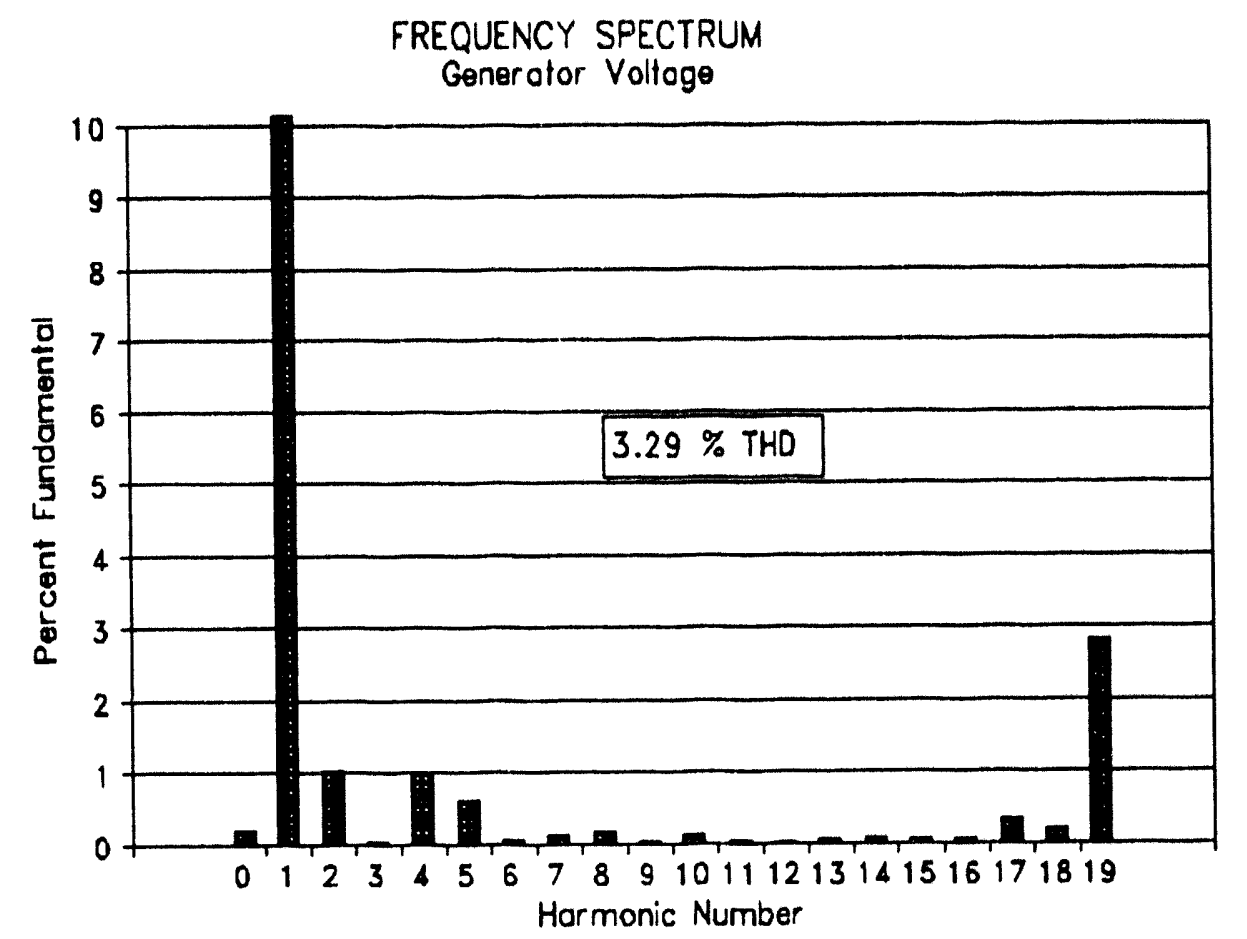

Fig. 4-13. Frequency spectrum for phase A generator voltage with 200 A GIC.

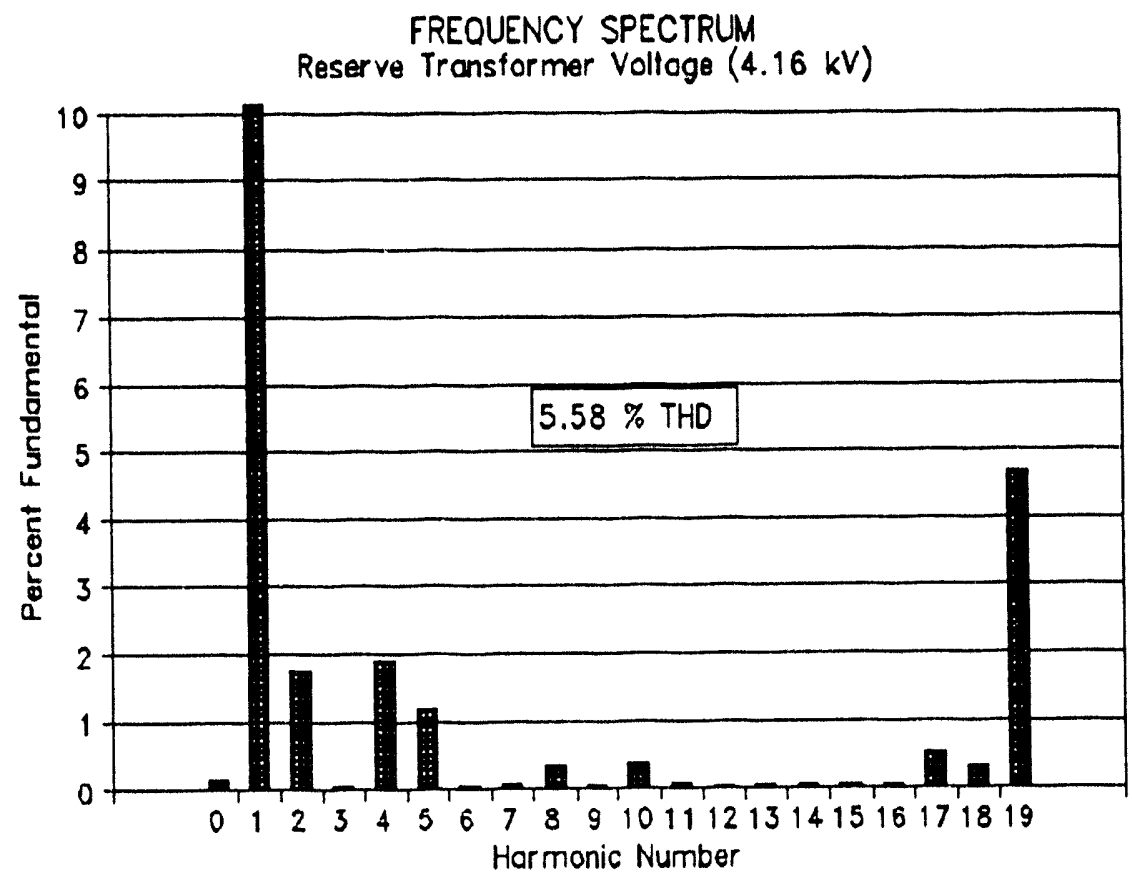

Fig. 4-14. Frequency spectrum for phase $A$ of reserve station service transformer voltage on LV side $(4.16 \mathrm{kV})-200 \mathrm{~A} \mathrm{GIC}$. 
FREQUENCY SPECTRUM

$120 \mathrm{~V}$ Emergency System Volloge

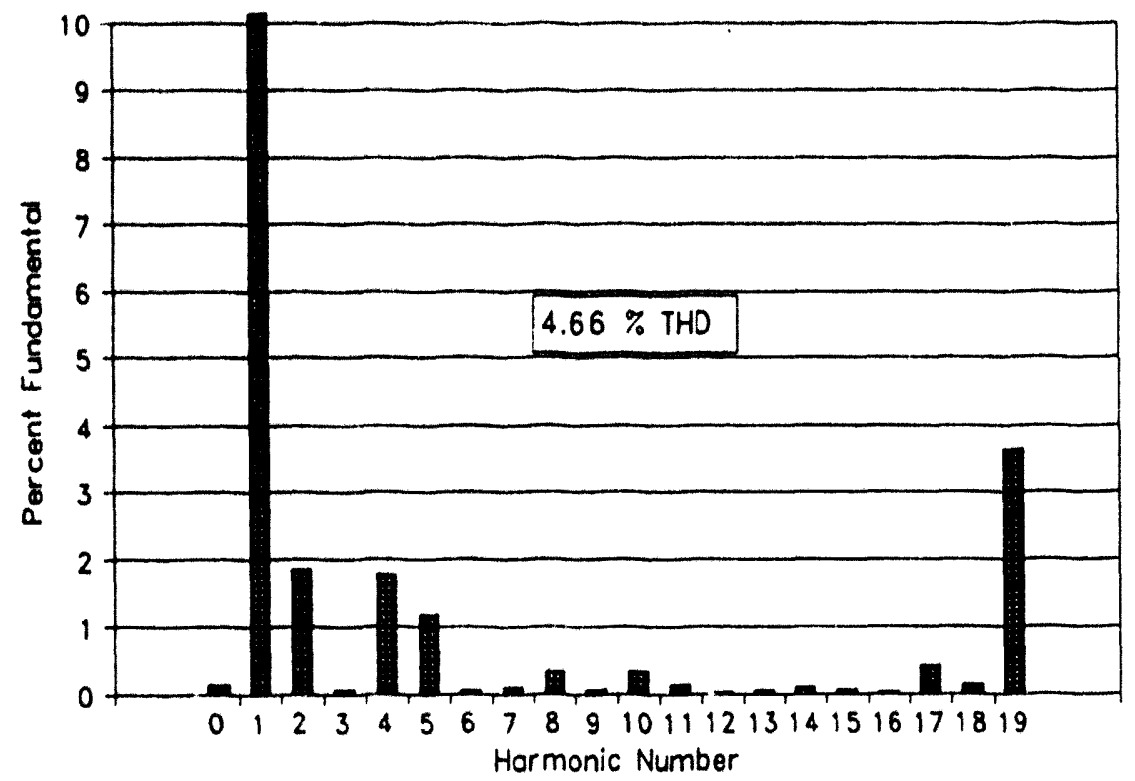

Fig. 4-15. Frequency spectrum of $120 \mathrm{~V}$ emergency system voltage with $200 \mathrm{~A}$ GIC.

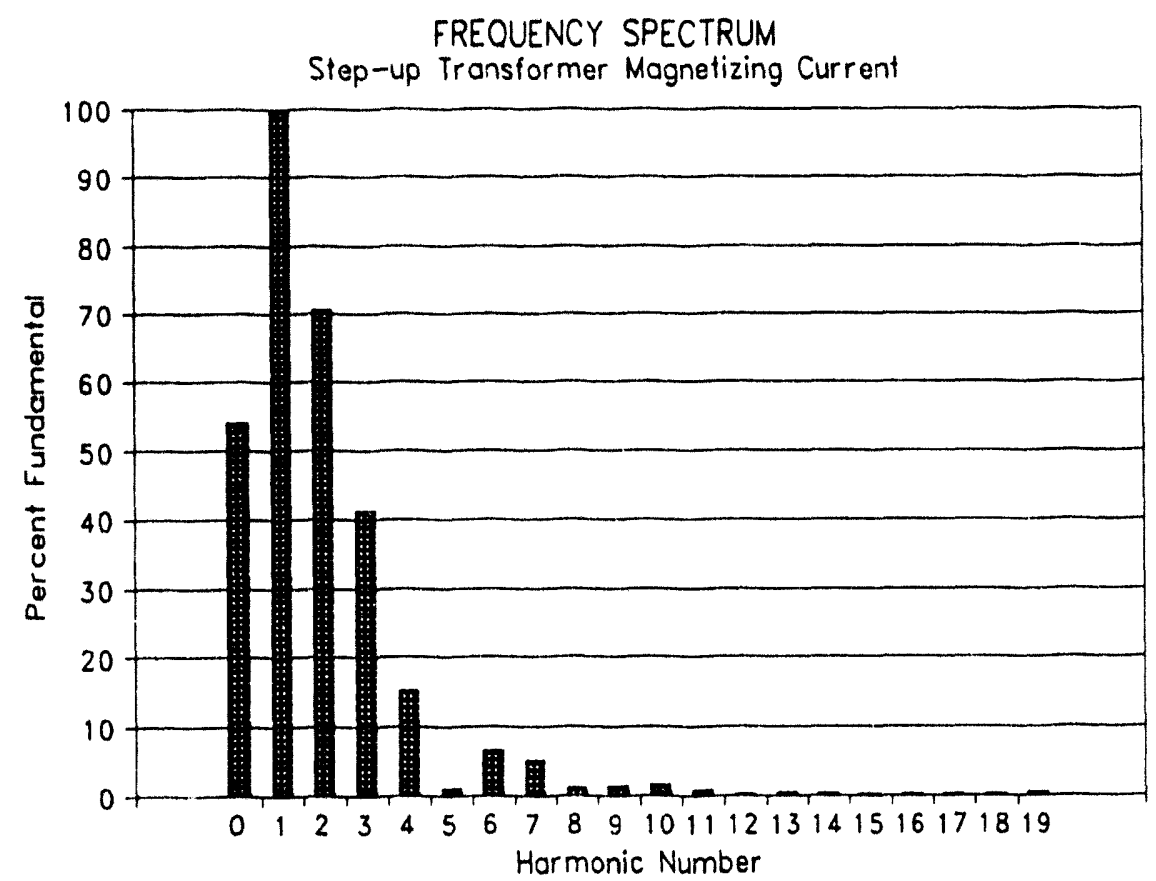

Fig. 4-16. Frequency spectrum for step-up transformer magnetizing current with 500 A GIC.

Figures F-19 and F-20 show the simulated voltages for the selected $600 \mathrm{~V}$ and $120 \mathrm{~V}$ buses in the system, subjected to $500 \mathrm{~A}$ GIC. Again the nonsafety-related voltages appear nearly sinusoidal, while the emergency system voltages are visibly distorted. Fig. 4-17 shows the frequency spectrum for the 120 $V$ emergency system voltage with 500 A GIC. Comparing with Figs. 4-12 and 4-15, shows the pronounced 
FREQUENCY SPECTRUM

$120 \mathrm{~V}$ Emergency System Voltage

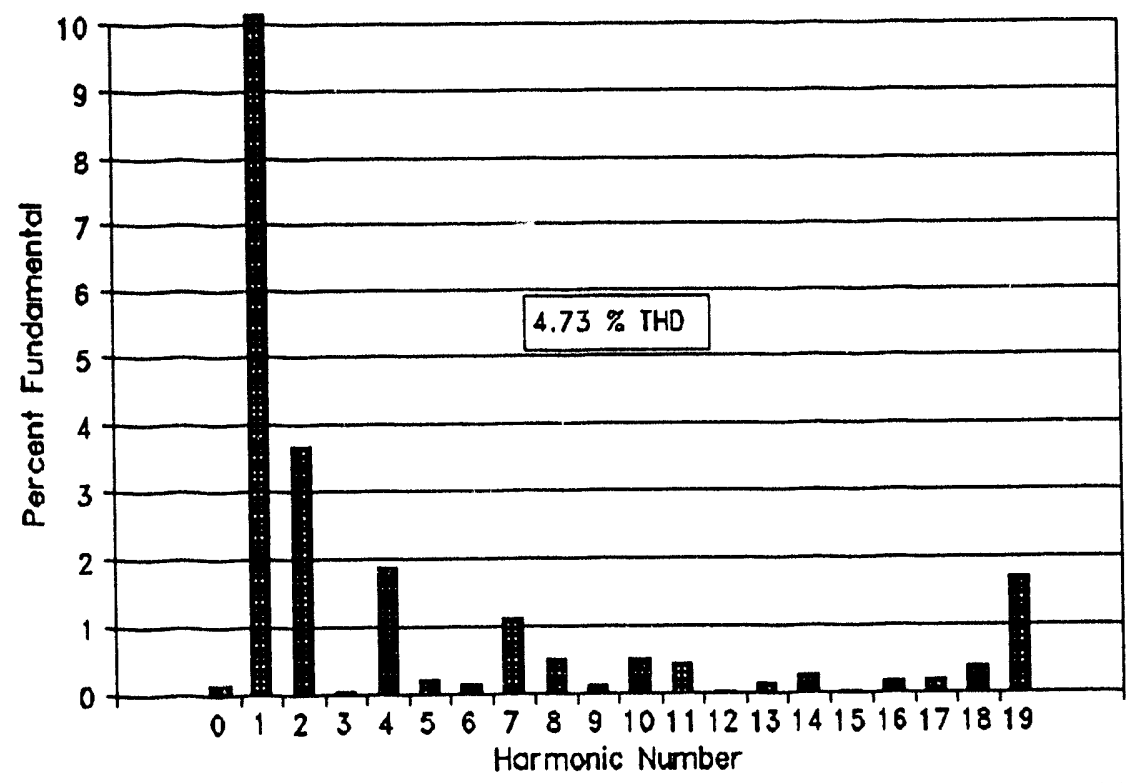

Fig. 4-17. Frequency spectrum for $120 \mathrm{~V}$ emergency system voltage with $500 \mathrm{~A}$ GIC.

shift in the voltage harmonics toward the lower frequencies at the $120 \mathrm{~V}$ level, while the THD shows only a slight increase to $4.73 \%$

Figure 4-18 summarizes the effect of different GIC magnitudes on the harmonic levels at various points in the simulated system. The THD is plotted versus transformer neutral current for the three cases considered. As indicated in the graph, harmonics generally increased with neutral current at low to moderate levels of GIC, but tended to fall off at higher GIC levels, due mainly to the shift in frequency spectrum of the transformer magnetizing currents. It is difficult to generalize from these results, which were obtained for one specific system in a specific operating condition. However, this example does reveal the complexity of the problem, and that it may be inaccurate to assume that harmonic levels are always proportional to the GIC magnitude.

\subsubsection{Coupling effect of nonsafety-related system with the emergency system}

The next study considered in the simulation, concerned the coupling at the transmission level of the nonsafety-related system with the emergency system. In the previous case the $115 \mathrm{kV}$ offsite supply, which feeds the emergency system through the reserve station service transformers, emanates from the local $345 \mathrm{kV}$ substation, which also connects to the main $345 \mathrm{kV}$ line out of the plant. Since the previous case study revealed the saturation sensitivity of the reserve station service transformer to relatively low GIC levels, it seemed prudent to make further studies assuming that no coupling exists that would limit dc neutral current in the reserve station service transformer. Therefore the simulation was modified to include a separate $115 \mathrm{kV}$ overhead line to this transformer, supplied from an independent source. The transmission line was assumed to be 50 miles long, using a tower configuration and conductor data given in Appendix E. The GIC level in both the step-up transformer and the reserve station service transformer was assumed to be $100 \mathrm{~A}$. 
TOTAL HARMONIC VOLTAGE DISTORTION

For Different GIC Levels

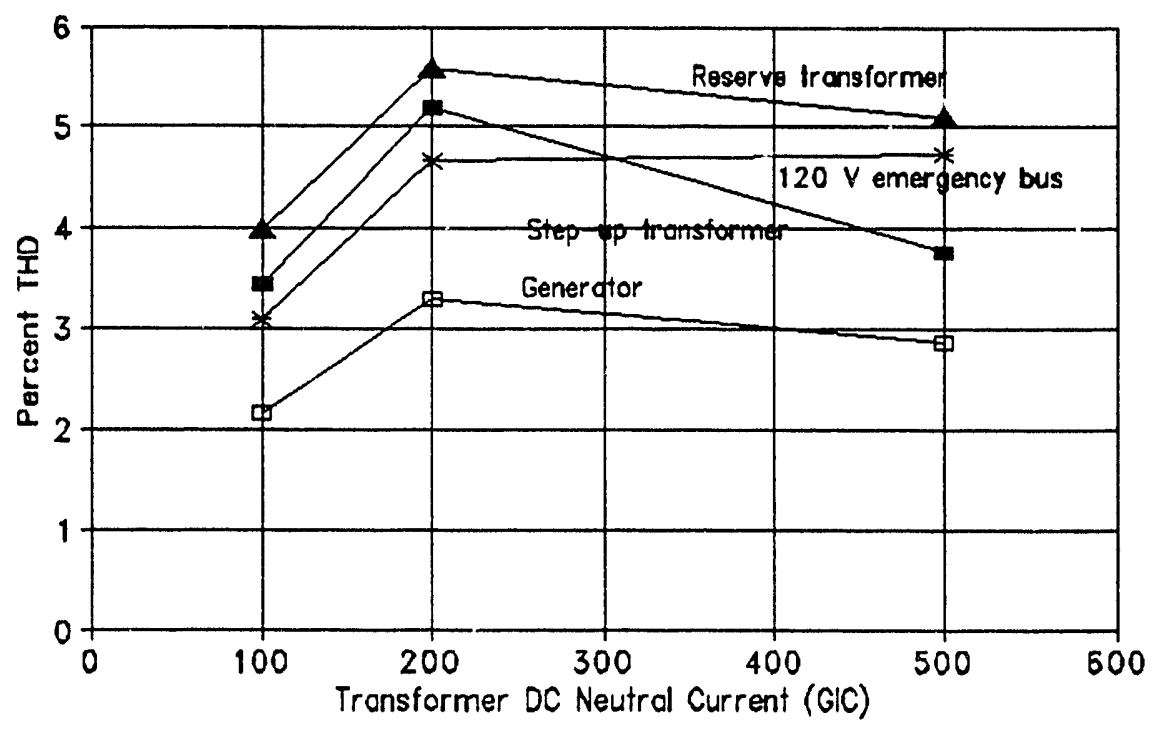

Fig. 4-18. Comparison of THD for different GIC levels.

Figure F-21 shows the magnetizing current in the reserve station service transformer for this new case, which is nearly double the previous case with a total GIC of $100 \mathrm{~A}$. Figure 4-19 shows the frequency spectrum for the A-phase magnetizing current pulses, which again is a rich source of low frequency harmonics. Figure F-22 confirms the average neutral current of approximately $100 \mathrm{~A}$ in the step-up transformer and also in the reserve station service transformer. Figure F-23 shows the reserve station service transformer voltages on the $4.16 \mathrm{kV}$ side, and Fig. 4-20 shows the frequency spectrum of the phase A waveform. The voltage waves are highly distorted, and the spectrum plot confirms this observation showing a variety of strong harmonics present in the wave over a wide frequency range. Comparing with Figs. 4-11 and 4-14, we see this spectrum is quite different than in the previous case study. The overall harmonic level is also significantly larger than before, with a THD of $8.93 \%$. Fig. F-24 shows the normal and emergency system voltages at the selected $120 \mathrm{~V}$ buses, and again the emergency system voltage is highly distorted. This is also verified by the frequency spectrum plot of this voltage, shown in Fig. 4-21, which reveals a wide range of relatively strong frequency components. The spectrum in Fig. 4-21 is similar to that in Fig. 4-20, with only a slight attenuation of harmonics from the $4.16 \mathrm{kV}$ level to the $120 \mathrm{~V}$ level. The overall harmonic level is also only slightly reduced, with a THD of $8.23 \%$.

\subsubsection{Effect of disconnecting the station service transformer}

The next case study considered was for plant operation in one stage of the start-up mode. In this case it was assumed that the normal station service transformer is disconnected from the normal onsite distribution system. Instead, the reserve station service transformers are assumed to carry the entire onsite load, including the nonsafety-related and the emergency system loads. Therefore, the only source of harmonics in the plant are from a GIC flow through the grounded neutrals of the reserve station servive transformers. Even if GIC flows through the step-up transformer there is no path for harmonics to el er the station. Referring to the simulation model in Fig. 4-5, this study was performed by opening the breakers from the normal station service transformer $\mathrm{T} 2$ to the $13.8 \mathrm{kV}$ buses B001 and B003, then 


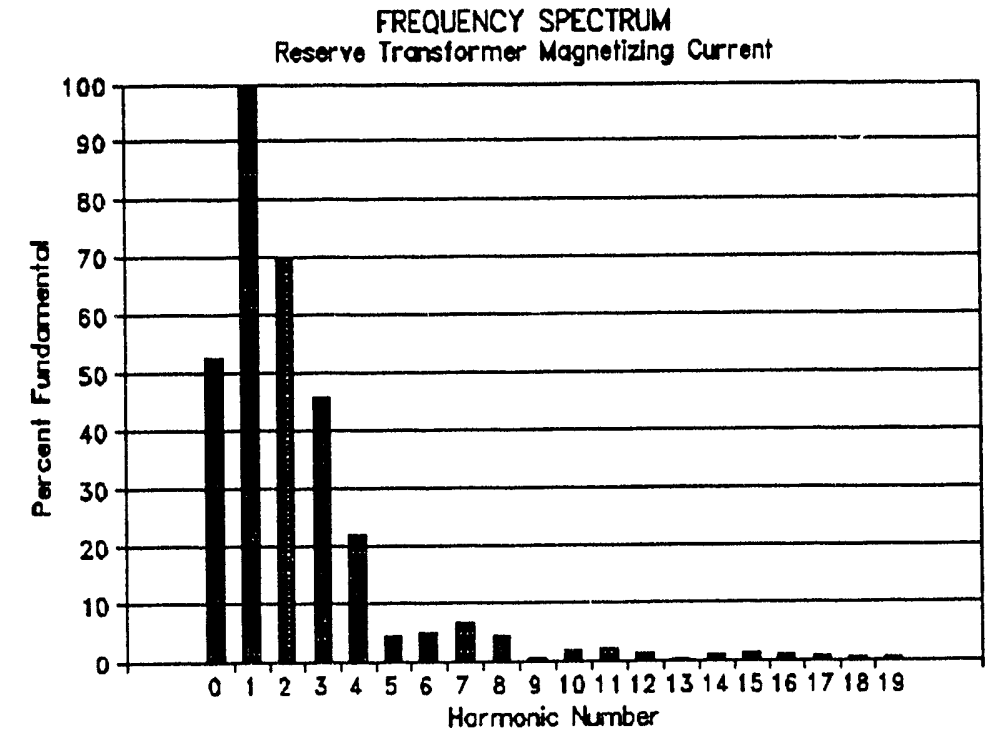

Fig. 4-19. Frequency spectrum of the magnetizing current in the reserve station service transformer, with separate $115 \mathrm{kV}$ line.

closing the NO breaker from the reserve station service transformer T3 to bus B003. One additional feature was also added to the simulation: a 0.15 pu source reactance was placed in series with the remote voltage source at the far end of the $345 \mathrm{kV}$ line. This is to model the finite short circuit capacity of the remote source. It may have the effect of increasing harmonic levels in the station, by further blocking harmonic current flow into the transmission system.

Figure F-25 shows the reserve station service transformer magnetizing currents for the assumed start-up mode of operation, with a GIC of $50 \mathrm{~A}$ at the $345 \mathrm{kV}$ transmission level. Since the GIC appears at this high voltage level, and the magnetizing current is referred to the $4.16 \mathrm{kV}$ winding of the transformer, the current pulses are higher than any previous case for the reserve station service transformer. Based on previous observations, this would seem to imply a potentially high harmonic level at the lower frequencies, unless some resonant condition develops. This is confirmed by Fig. 4-22, which shows the frequency spectrum of the A-phase transformer magnetizing current.

Figure F-26 shows the voltage waves on the $4.16 \mathrm{kV}$ side of the reserve station service transformer, and Fig. 4-23 shows the corresponding frequency spectrum of the A-phase voltage. Distortion is clearly visible in these waveforms, and the spectrum reveals several low frequency components and a partial resonance at the tenth harmonic. This is probably due to an interaction between the station impedance and the transmission line. The THD at the $4.16 \mathrm{kV}$ voltage level is $4.94 \%$. Figures F-27 and F-28 show normal system and emergency system voltages at the $600 \mathrm{~V}$ and $120 \mathrm{~V}$ levels. Again the emergency system voltages are visibly distorted. Figure 4-24 shows the frequency spectrum of the $120 \mathrm{~V}$ emergency system voltage, which has similar characteristics to Fig. $4-23$ for the $4.16 \mathrm{kV}$ level. This again indicates very little harmonic attenuation through the lightly-loaded emergency system. 
FREQUENCY SPECTRUM

Reserve Transformer Voltage $(4.16 \mathrm{kV})$

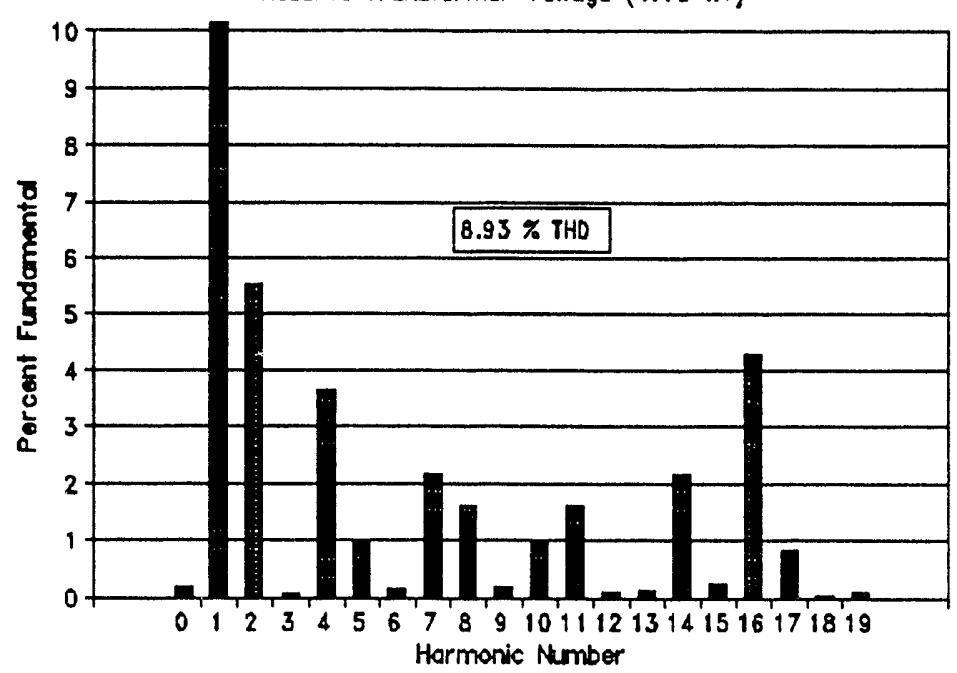

Fig. 4-20. Frequency spectrum of A-phase reserve station service transformer voltage on $4.16 \mathrm{kV}$ side, with separate $115 \mathrm{kV}$ line.

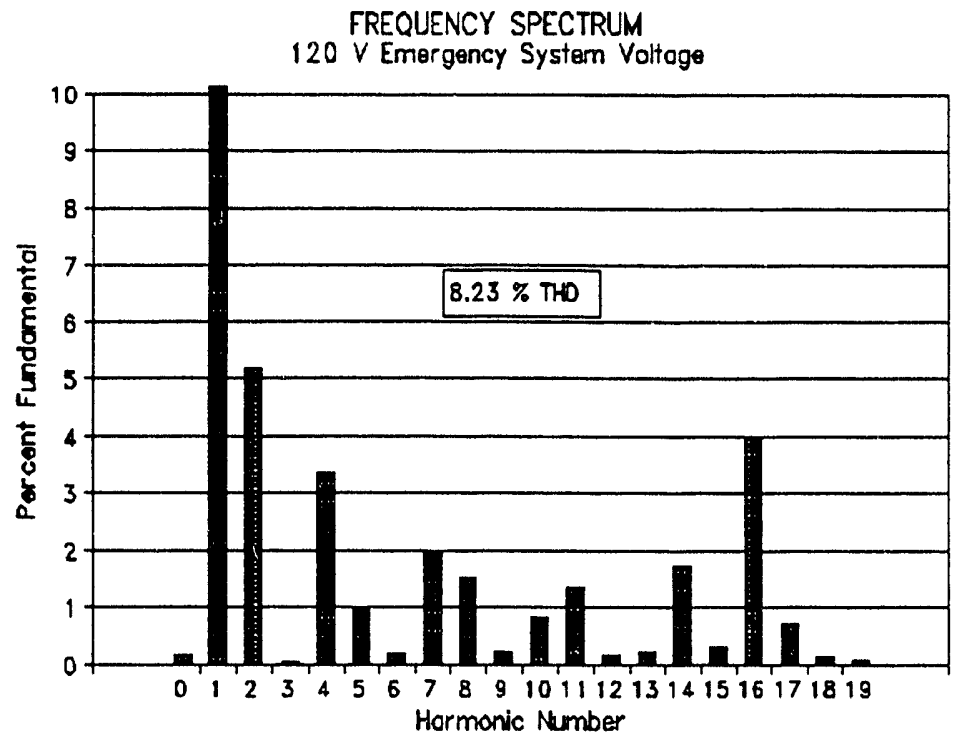

Fig 4-21. Frequency spectrum of $120 \mathrm{~V}$ emergency system voltage, with separate $115 \mathrm{kV}$ line to reserve station service transformer. 


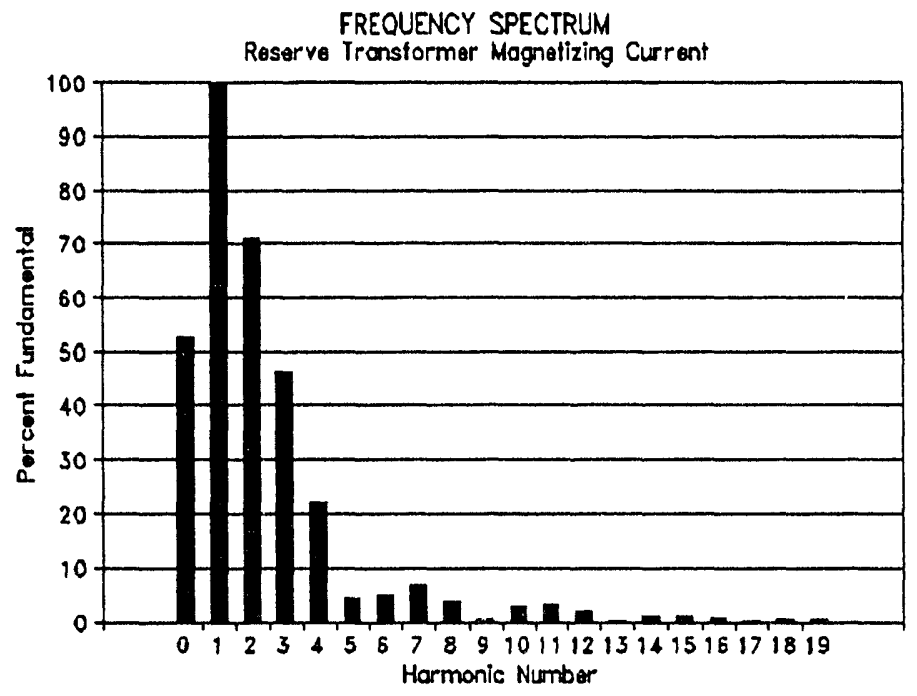

Fig. 4-22. Frequency spectrum of the A-phase magnetizing current in the reserve station service transformer, during start-up mode.

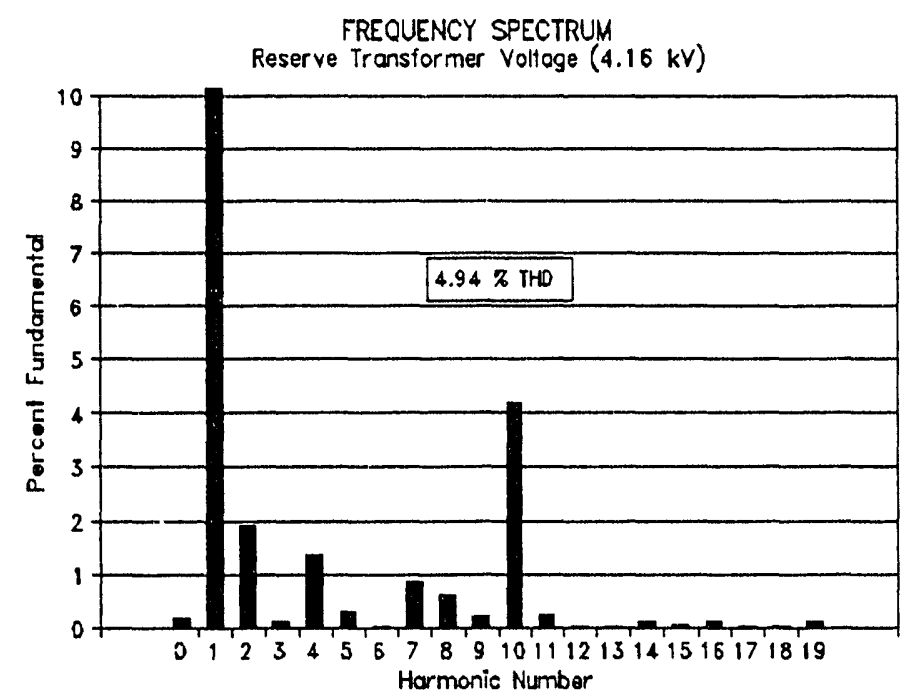

Fig. 4-23. Frequency spectrum for the A-phase reserve station service transformer voltage (4.16 $\mathrm{kV}$ side), during start-up mode. 


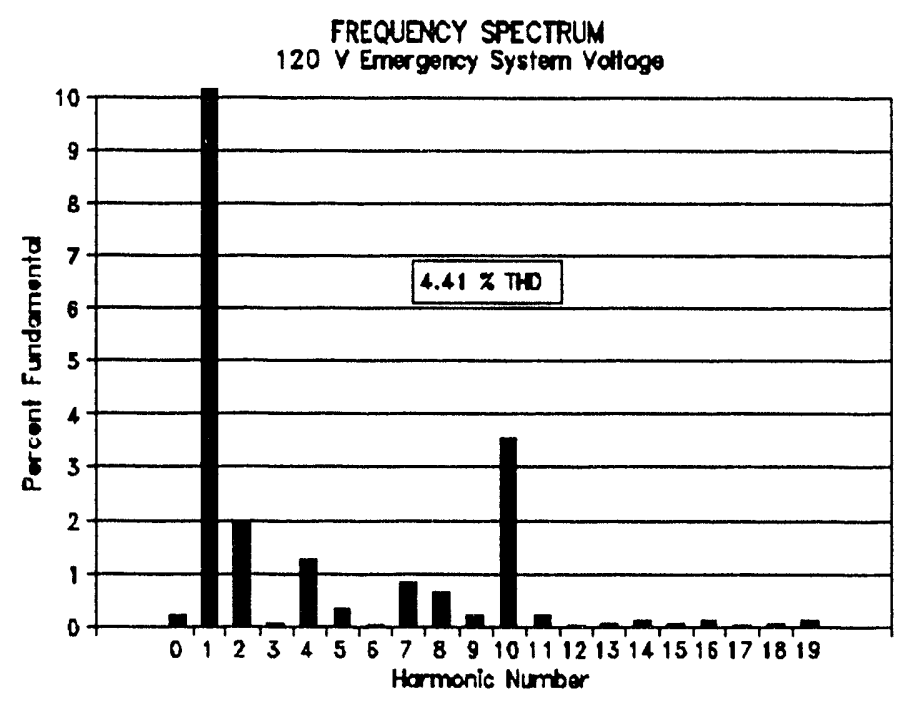

Fig. 4-24. Frequency spectrum of $120 \mathrm{~V}$ emergency system voltage, during start-up mode.

\subsection{Summary}

EMTP (ElectroMagnetic Transients Program) simulations were performed to investigate the onsite effect of geomagnetic induced currents (GIC) on in-plant electrical distribution system in a nuclear power plant. Models were developed to simulate the behavior of a typical electrical distribution system within the plant for the nonsafety-related and the safety-related (emergency) circuits. The offsite transmission system was also modeled. Model data were derived from FSAR reports, T\&D reference books, and other published literature. Studies thus far have concentrated mainly on steady-state harmonic effects at the various voltage levels in the system. Voltage harmonics levels were evaluated for several system configurations and operating conditions. Total Harmonic Distortion (THD) levels in the range of $4-9 \%$ have been observed in the simulation, at selected low voltage buses, for certain assumed conditions and GIC levels. The emergency circuits appear to be more susceptible to high harmonics due to the normally light load conditions.

For the particular electric distribution system configuration studied, the harmonic levels at various plant electrical bus level are not necessarily proportional to the GIC magnitude. The total harmonics distortion observed at various points in the plant disturbance system generally increased with neutral current at low to moderate levels of GIC, but tended to fall off at higher GIC levels. Also, high voltage level safety related buses being light loaded, except under emergency operating conditions, may be more vulnerable to higher THD than their non-safety-related counter part. Generally the harmonic levels produced are proportional to the magnitude of the transformer saturation currents. The magnitude of these saturation currents depend on the average neutral current, which in turn, depends mainly on the offsite line and ground resistances and the ESP produced by the geomagnetic disturbance. Furthermore, the resulting current flow depends on the impedances of both the onsite and offsite system. This final dependance was further studied in the next section. 


\section{EFFECTS OF TRANSMISSION LINE CONFIGURATION}

\subsection{Frequency Analysis of Case Studies}

EMTP simulation studies and Fourier analysis of the waveforms taken from the previous simulations showed that the harmonic levels present on the plant distribution buses depends on several factors. These include; (a) the magnitude of the GIC, (b) the loading of the distribution buses, (c) the transformer saturation characteristics, and (d) the transmission line configuration, line length and magnitude of remote source impedance. Among these factors the transmission line characteristics may be the most important in determining both the magnitude and dominant frequency of harmonic currents forced into the station circuits. This is best explained by referring to the circuit analogy of the process suggested by Fig. 4-6 in the previous section. The step-up transformer and/or the reserve station service transformer, whichever is fed through grounded wye-connected windings from the line side, acts essentially as a current source of various harmonic frequencies. These harmonic currents may flow into either the transmission system or the station distribution system, depending on the relative impedances of the two sides of the circuit. The effective harmonic current source amplitude falls off sharply with frequency, as both the distribution circuit impedance and transmission line impedance generally increase with frequency. However, the transmission line may reach its resonant point near one of these higher harmonics, which results in a very sharp increase in line impedance. This causes most of the harmonic current at that frequency and nearby frequencies to be forced into the distribution system. The transmission line thus acts like a frequency-sensitive valve, which acts to either drain off and dissipate harmonic currents in the transmission network, or block these currents and force them into the station circuits. Once the harmonic currents enter the distribution network, the harmonic voltage levels at the various buses depends on the relative circuit impedances. The dominant impedances in the distribution network are inductive, due to the various transtormer leakage reactances and the major loads. The network impedance therefore increases with frequency, and is generally higher under light loading conditions. Thus the harmonic voltages can be quite high at high frequencies, even if harmonic current injection is low, especially for light loads.

In order to test the above theory, the "Frequency Scan" feature of EMTP was used to compute transmission line impedance functions, as viewed from the excitation branch of the step-up transformer and/or the reserve station service transformer. These impedance functions were computed using the complete system models used in the previous time simulations, by injecting harmonic currents into the transformer excitation circuits, then measuring the harmonic voltages produced at the terminals of the transmission line. Since these impedance functions are computed using the total current injection, including that flowing into the distribution network, they are not true measurements of the line impedance, but serve only as a rough measure of the blocking ability of the line over the frequency spectrum of interest.

Appendix G contains selected results of the Frequency Scan study. Figure G-1 is a plot of the 50 mile, $345 \mathrm{kV}$ transmission line impedance function versus frequency, for normal station configuration and nominal system parameters. This plot shows a relatively sharp resonance near the $19^{\text {th }}$ harmonic, which explains the strong presence of this frequency component in Figs. 4-9 through 4-15, as well as in the simulated waveforms in Figs. F-4 through F-10 and Figs. F-13 through F-16.

Figure G-2 is a plot of the transfer ratio of the transmission line voltage to the step-up transformer magnetizing voltage. This ratio is computed using a Frequency Scan of the system with a 1 per unit harmonic voltage placed at the transformer excitation branch, then measuring the voltage response at the sending (local) end of the transmission line. In general the transfer ratio is a convenient 
measure of how the harmonic voltages are amplitied or attenuated at various locations in the system. In Fig. G-2 it shows that harmonic voltages at the transmission line generally increase with frequency, following its impedance profile, and eventually exceed the natural voltage drop at $60 \mathrm{~Hz}$ due to line loading.

Figures G-3 and G-4 are also voltage transfer plots at various levels in the nonsafety-related (normal) and the safety-related (emergency) distribution circuits. Figure G-3 shows that nonsafetyrelated harmonic levels generally fall off with frequency at the lower voltage levels, relative to the step-up transformer bus. On the other hand, Fig. G-4 shows that the emergency system harmonics tend to increase with frequency at all voltage levels, and eventually exceed the normal $60 \mathrm{~Hz}$ loading drop. This effect is mainly due to the difference in loading between the two circuits. The lightly loaded emergency system has relatively high (reactive) load impedances, which increase with frequency and produce higher harmonic voltages at all buses. The moderately loaded nonsafety-related system has lower load impedances and higher currents, which produce greater harmonic voltage drops across the various transformers as the frequency increases. The transfer ratios and above analysis help explain why greater harmonics were generally observed in the emergency system for all cases studied in the simulations. For example, compare the contrasting pair of waves in Figs. F-10, F-16 and F-24.

Figure G-5 shows a plot of the transmission line impedance functions versus frequency, for the 50 mile $345 \mathrm{kV}$ line and the 50 mile $115 \mathrm{kV}$ line, for the case of separate $115 \mathrm{kV}$ service with nominal system parameters. The $345 \mathrm{kV}$ line still resonates near the $19^{\text {th }}$ harmonic, while the $115 \mathrm{kV}$ line produces an even sharper resonance slightly above the $15^{\text {th }}$ harmonic. In this case the $19^{\text {th }}$ harmonic resonance has little effect on the distribution system, because very little excitation current is produced at that frequency. Also the potential effect of the $15^{\text {th }}$ harmonic resonance, which is a multiple of the $3^{\text {rd }}$ harmonic, is mitigated by the delta windings present in nearly all major transformers. However, the combined impedance of the two lines does produce signiticant harmonic flow into the station distribution system at several notable frequencies. This is corroborated by the Fourier analysis of the simulated waveforms of the reserve station service transformer voltage and the $120 \mathrm{~V}$ emergency system voltage, as shown in Figs. 4-20 and 4-21.

Figures G-6 and G-7 are voltage transfer plots at various levels in the nonsafety-related (normal) and the safety-related (emergency) distribution circuits, for the case of separate $115 \mathrm{kV}$ service to the station. Figure G-6 shows that nonsafety-related harmonic levels again fall off with frequency at the lower voltage levels, relative to the step-up transformer bus. On the other hand, Fig. G-7 shows that the emergency system harmonics fall off only slightly with frequency at all voltage levels. This effect is again due mainly to the difference in loading between the two circuits.

Figure G-8 shows a plot of the transmission line impedance function versus frequency, for the 50 mile $345 \mathrm{kV}$ line, during station start-up mode with nominal system parameters and remote source impedance included. The $345 \mathrm{kV}$ line now exhibits a double resonance near the $9^{\text {th }}$ and $10^{\text {th }}$ harmonics. In this case the $9^{\text {th }}$ harmonic resonance has little effect on the distribution system, because it is a multiple of the $3^{\text {rd }}$ harmonic, and is mitigated by the delta windings present in the various transformers. However, the $10^{\text {th }}$ harmonic line impedance does force significant harmonics into the station in this mode of operation. This is again corroborated by the Fourier analysis of the simulated waveforms of the reserve station service transformer voltage and the $120 \mathrm{~V}$ emergency system voltage, as shown in Figs. 4-23 and 4-24. 
Figures G-9 and G-10 are additional voltage transfer plots at various levels in the nonsafetyrelated (normal) and the safety-related (emergency) distribution circuits, for the start-up mode of operation. Figure G-9 again shows that nonsafety-related harmonic levels fall off with frequency at the lower voltage levels, while Fig. G-10 shows that the emergency system harmonics fall off only slightly with frequency at all voltage levels.

\subsection{Extended Case Studies}

From the above discussion it is clear that the transmission line configuration and its resonant frequency play a significant role in determining harmonic levels in the station distribution circuits. Moreover, the line length is the main parameter that determines the resonant frequency for a given line type. Since line length is generally not an adjustable parameter, but is determined by geographic conditions, various resonant frequencies are possible. The situation is further complicated since most transmission systems are interconnected networks, not a single line, therefore several different resonant frequencies are possible. Furthermore, it is possible that one or more of these resonant frequencies in the transmission system may be excited by a relatively strong harmonic component of the transformer excitation current. In the following case studies, the line lengths of the transmission system were modified from the original studies performed earlier, in order to further illustrate the influence of this important parameter. Appendix $\mathrm{G}$ contains the results of Frequency Scan studies for these extended cases, and Appendix $\mathrm{H}$ contains the corresponding EMTP simulation results and Fourier analysis of the waveforms obtained.

Figure G-11 shows the impedance function for the $345 \mathrm{kV}$ transmission line with the line length changed to 42 miles and a remote source impedance added at the far end of the line. Otherwise, the system is in the normal operating mode with nominal system parameters, as for the case shown in Fig. G-1. Note that the main resonant peak has moved to near the $17^{\text {th }}$ harmonic with a secondary peak near the $16^{\text {th }}$ harmonic. Neither of these frequencies will be affected by the delta windings, so significant harmonics should be present at these frequencies, if sufficient GIC-induced excitation current is present. Figures $\mathrm{H}-1$ through $\mathrm{H}-8$ show the simulation results obtained for this case with normal station operation and 100 A GIC. Figures $\mathrm{H}-1$ through $\mathrm{H}-4$ show that the transformer excitation currents have very similar characteristics to those obtained earlier for the 50 mile transmission line, with slightly reduced excitation at the higher harmonics in the step-up transformer. Figures $\mathrm{H}-5$ through $\mathrm{H}-8$ show the characteristics of the reserve station service transformer voltage $(4.16 \mathrm{kV})$, and the $120 \mathrm{~V}$ normal and emergency system voltages. As expected, the dominant harmonics due to the changed line resonance are the $16^{\text {th }}$ and $17^{\text {th }}$. The THD is also increased from the earlier case at both these voltage levels.

Figure G-12 shows the impedance function for the $345 \mathrm{kV}$ transmission line with the line length changed to 49.07 miles and no remote source impedance. Otherwise, the system is in the normal operating mode with nominal system parameters, again like the case shown in Fig. G-1. In this case, however, the main resonant peak is tuned exactly to the $19^{\text {th }}$ harmonic, which should result in increased harmonic levels at that frequency. Figures $\mathrm{H}-9$ through $\mathrm{H}-16$ show the simulation results obtained for this case with normal station operation and $100 \mathrm{~A}$ GIC. Figures $\mathrm{H}-9$ through H-12 again show that the transformer excitation currents have very similar characteristics to those obtained earlier for the 50 mile transmission line. Figures $\mathrm{H}-13$ through $\mathrm{H}-16$ show the characteristics of the reserve station service transformer voltage $(4.16 \mathrm{kV})$, and the $120 \mathrm{~V}$ normal and emergency system voltages. As expected, the dominant harmonic due to the slightly changed line length is still the $19^{\text {th }}$. The apparent waveform distortion and the THD is also signiticintly increased from the earlier case at both these voltage levels. 
Figure G-13 shows the impedance functions for the system with a 50 mile $345 \mathrm{kV}$ transmission line, and a separate $115 \mathrm{kV}$ line with the line length changed to 69.55 miles. Otherwise, the system is in the normal operating mode with nominal system parameters, like the case shown in Fig. G-5. Note that the resonant peak of the $115 \mathrm{kV}$ line has moved from near the $15^{\text {th }}$ to the $11^{\text {th }}$ harmonic, which should result in significantly increased harmonic levels, especially since this new resonant frequency will not be affected by the transformer delta windings. Figures $\mathrm{H}-17$ through $\mathrm{H}-22$ show the simulation results obtained for this case with normal station operation and $100 \mathrm{~A}$ GIC. Figures $\mathrm{H}-17$ and $\mathrm{H}-18$ show that the reserve station service transformer excitation current has similar characteristics to those obtained earlier for the 50 mile $115 \mathrm{kV}$ transmission line. Figures $\mathrm{H}-19$ through $\mathrm{H}-22$ show the characteristics of the reserve station service transformer voltage $(4.16 \mathrm{kV})$, and the $120 \mathrm{~V}$ normal and emergency system voltages. As expected, the dominant harmonic due to the changed length of the 115 $\mathrm{kV}$ line is the $11^{\text {th }}$, although there still remains significant levels of other harmonics. The apparent waveform distortion and the THD (about 12\%) is also significantly increased from the earlier case at both voltage levels.

Figure G-14 shows the impedance function for the $345 \mathrm{kV}$ transmission line for the start-up mode of operation and with the line length changed to 42.38 miles. The system is operating with nominal system parameters, including a remote source impedance, like the case shown in Fig. G- 8 . In this case, with the shorter line, the main resonant peak moves up to the $11^{\text {th }}$ harmonic with a secondary peak at a slightly lower frequency, which should result in increased $11^{\text {th }}$ harmonic levels in the station. Figures $\mathrm{H}-23$ through $\mathrm{H}-28$ show the simulation results obtained for this case with the start-up mode of operation and $50 \mathrm{~A}$ GIC. Figures $\mathrm{H}-23$ and $\mathrm{H}-24$ again show that the transformer excitation currents have similar characteristics to those obtained earlier for the 50 mile transmission line. Figures $\mathrm{H}-25$ through $\mathrm{H}-28$ show the characteristics of the reserve station service transformer voltage $(4.16 \mathrm{kV})$, and the $120 \mathrm{~V}$ normal and emergency system voltages. As expected, the dominant harmonic due to the changed line length is moved up from the $10^{\text {th }}$ to the $11^{\text {th }}$. The visible waveform distortion and the THD is also significantly increased from the earlier case at both these voltage levels.

\section{$5.3 \quad$ Summary}

The harmonic currents may flow into either the transmission system or the inside power plant distribution system, depending on the relative impedances of the two sides of the circuit. In general, these impedances increase with frequency, while the effective harmonic current source amplitude falls off sharply with frequency. Depending on the line configuration, line length and magnitude of remote source impedance, this transmission line may reach its resonant point near one of the higher harmonics, which results in a very sharp increase in line impedance. This causes most of the harmonic current at the resonant frequency and adjacent frequencies to be forced into the distribution system. From this extended studies, it can be concluded that the transmission line and the network impedance plays a significant vote in determining the extent of the harmonics generated in the transformer that gets forced into the plant distribution system. But, the variations in the transmission network and switchyard configurations are too many to permit a systematic analysis to generate a generalized rule to quantify the effect. It is reasonable to postulate that for any given network configuration, there will always be at least one resonant frequency that will be executed by a relatively strong harmonic component of the transformer excitation current. Therefore it is important that the configuration at each plant be evaluated. 


\section{EFFECTS OF GIC HARMONICS ON PLANT EQUIPMENT}

\subsection{Harmonics in Power Systems}

For many years harmonics have been reported to cause operational problems in power systems and equipment [14]. Some of the effects include:

- $\quad$ Capacitor bank failure

- Interference with ripple control and power line carrier

- Misoperation in remote switching and load control

- $\quad$ Metering errors

- Excessive losses and heating in electric machines

- Resonant overvoltages and excessive currents in the network

- Dielectric breakdown of insulated cables

- Interference with telecommunications systems

- Signal interference and misoperation of protective relays

- Interference with large motor drives and excitation systems

- Electromechanical oscillations in large AC machines

These effects are dependent on the type of harmonic source, its location in the power system, and the corresponding network characteristics. It is clear, however, that harmonics can effect a wide variety of the components in the power system, both inside and outside of the plant. Geomagnetically induced currents (GIC) are just one of many sources of harmonics, but they produce a somewhat unique frequency spectrum, which must be examined for its effect on harmonic-sensitive equipment in the plant.

For purposes of this study, the Uninterruptible Power Supply (UPS) system and protective relays with specific emphasis on the undervoltage protective relays were chosen for further evaluation. The rationale behind their choice is that:

a. UPS system is a vital component in the plant protection system (PPS). Any impact on its performance due to GIC could have serious consequences on the electronic components that make up the PPS.

b. Undervoltage relays are used in effecting a transfer from the off-site to the on-site power source in the event of a loss voltage or degraded voltage condition on the emergency busses. This application is very critical since any GIC induced effect that either masks a truly existing undervoltage condition or affects (e.g., introduce a time or setpoint lag) the performance of the relay otherwise, could challenge one of the key assumptions (diesels to start and carry the required loads within a prespecified time limit) used in accident analysis. 


\subsection{Impact on Uninterruptible Power Supply System}

An Uninterruptible Power Supply (UPS) System provides an uninterrupted source of power to critical or vital loads such as instrumentation systems, plant protection systems and engineered safety feature systems. In addition to providing an uninterruptible power source, UPS also provides isolation from the utility plant distribution systems and associated line voltage variations.

The most common configuration in which an UPS is used is called the "Float" or "Reverse Power" configuration as shown in Figure 6.1. This system utilizes AC power from the plant AC distribution system and converts it to DC through a batter charger. The regulated DC power from the battery charger is supplied to the battery system to maintain the battery fully charged, and to the invertor to supply the load. An invertor usually consists of three basic components as follows:

1. A switching bridge which converts the DC power to AC square wave power.

2. A filter network to refine the $\mathrm{AC}$ square waves to low distortion $\mathrm{AC}$ sine waves, and

3. A control logic which provides control to the switching bridges.

The invertor converts the $\mathrm{DC}$ back to $\mathrm{AC}$ which is fed to the load through a regulator, filter and static transfer switch. The regulator and filter ensures that the output voltage is maintained constant and noise free. The static transfer which also receives AC power from the plant AC distribution system, under normal conditions, passes the invertor output AC power to load. Upon failure of the invertor, or a fault on the load side leading to an excessive overload condition of the invertor or its output regulator, the static transfer switch automatically transfers the load to the plant AC power source, thus maintaining continuity of the power input to the loads. The transfer time of the static transfer switch ranges from virtually zero for an UPS using the ferro-resonant or constant voltage transformer type output filter to about 4 to 5 milliseconds for the pulse width modulated system. A manual bypass switch (usually a make-before-break switch) is used as shown in the figure to provide a means of transfer to the plant AC power source for maintenance of the UPS, or if the static transfer switch becomes inoperable.

Two commonly used UPS systems in nuclear plants are the ferro-resonant transformer type and the Pulse Width Modulated (PWM) type systems. In a ferro-resonant transformer type system (Fig. 6.2), a square wave is produced in the primary from the DC input be switching a set of silicon controlled rectifiers. Impulses in the secondary activate a resonant tuned saturating magnetic circuit and $A C$ capacitor combination. The ferro-resonant transformer operates in saturation thus providing its own regulation and harmonic neutralizing. In addition, it is also inherently current limiting when over loaded. It is a simple system.

In a pulse width modulated invertor system (see Fig. 6.3), a set of SCR bridges are switched to $\mathrm{p}$ iduce a train of square wave pulses which approximate an $\mathrm{AC}$ sine wave. An output filter is employed to iurther smooth the approximate sine wave output from the $\mathrm{DC}$ to $\mathrm{AC}$ invertor. The feedback control logic systems provide voltage control and current limiting.

The selection of the type of UPS system is based among others on the transient response, efficiency, output quality, reliability, and maintenance economics. 
In the float configuration (see Fig. 6.1), if $\mathrm{AC}$ power to the rectifier and battery charger is lost, the batteries automatically supply DC power to the invertor since the charger is connected in a float mode to the battery bus. Under normal conditions, the battery charger section of the UPS provides complete isolation from the plant AC system through an input isolation transformer. As shown during earlier simulations, there is no dc offset component in any of the transformers within the plant distribution system. In addition, the DC output from the charger is regulated and has current limiting capabilities since the output voltage regulation of the charger is designed to meet the stringent demands for a precise charging yoltage for the battery. Therefore, any change in the average or the rms input voltage of the plant AC power source resulting from GIC induced high harmonic distortion will have insignificant effect on the load side as long as the input voltage remains within the specifications of the invertor (105 to $140 \mathrm{VDC}$ ). Thus the only condition under which the GIC induce high total harmonic distortion would be of concern is when a static switch transfer from the UPS source to the plant distribution system source takes place. It is believed that the probability of such transfer demand on the static transfer switch occurring simultaneously during a window of high solar magnetic disturbance is very low. the rationale for this judgement is that it would require a combination of two or more infrequent occurrences to take place simultaneously. For example under one scenario, it would require a failure of an UPS system occurring precisely during a window (usually about 30 minute to one hour maximum) of high solar magnetic disturbance resulting in high GIC at a particular geographic location.

Therefore, it was decided that no further study of the effect of GIC induced high harmonic distortion of the UPS system or the 120 Vac system is warranted at this time.

\subsection{Impact of Harmonics on Protective Relays}

Both electromechanical and static relay performance are affected by distorted waveforms containing harmonics [15]. However, there appears to be a lack of performance requirements in the IEEE relay standards regarding waveform distortion and harmonics. Relay manufacturers calibrate their relays and publish performance data based mainly on sinusoidal currents and voltages. The variety of relay designs and operating techniques used by these vendors makes it difficult to arrive at meaningful standards of performance, when harmonics are involved. Different designs for the same relay function may behave quite differently if subjected to distorted non-sinusoidal measurements.

Protective relays generally do not respond to one particular parameter such as the RMS value or the fundamental frequency component. Also the performance of a relay to a range of single frequency inputs is not the same as that for a distorted wave containing these frequencies. Superposition does not apply. In addition, multi-input relays may be more unpredictable than single-input relays in the presence of harmonics. Relay response under distorted conditions may vary among relays having the same nominal fundamental frequency characteristics, not only among different manufacturers, but also among different design vintages from the same manufacturer.

\section{Electromechanical Relays}

Electromechanical relays are largely single-input, single-phase devices. These include induction disk designs for time delays or induction cup designs for instantaneous operation. Induction disk designs are usually optimized for one frequency, and therefore will be less efficient at any other frequency. This type of relay will tend to be less sensitive and operate more slowly when the input waveform contains harmonics. Counteracting this effect is the synchronous speed of the induction disk, which is proportional to the input frequency. Thus the relay may operate faster when the input contains a strong component 
of higher harmonics. Each relay manufacturer has a unique torque producing design, so that similar relays from different manufacturers may respond to distorted signals differently.

Instantaneous overcurrent relays of the induction cup design will usually have a frequency response similar to the induction disk type. However, plunger type instantaneous current relays are generally independent of harmonic effects, and respond primarily to the RMS value of the current measurement. On the other hand, voltage relays tend not to respond to the total RMS value, but rather to the fundamental component of the measured input wave. This is due to the inherent current limiting effect of the coil impedance at harmonic frequencies.

\section{Static Relays}

Static relays have at least as many design variations among manufacturers as electromechanical relays. Single-input static relays, such as a time-overcurrent relay, may be designed to respond to either peak value, average value, or RMS value of the input waveform. Each has its own characteristics and design merits for a specific application. Response to non-sinusoidal waveforms may vary significantly among basic designs, even though each may be calibrated to provide identical performance with the expected single-frequency sinusoidal waveform. Even RMS responsive relays may have frequencyweighted networks that modify the true RMS performance in the presence of harmonics. Waveshape can affect both the pickup value and time-delay characteristics, and the method used to shape the timecurrent characteristic will influence the basic response of the relay. Also, when nonlinear elements such as diode networks are used for curve shaping, the relays tend to respond more to peak values. When zero crossing time is utilized in the measurement technique as in frequency relays, distortion can significantly affect the relay accuracy.

Static overcurrent relays may use saturable transformers in the input circuit to limit the energy transfer and help shape the time-overcurrent curve. This may cause internal waveshape distortion which can interact with already distorted input signals and thus affect overall relay response. Most static relays also use small input transformers to transform the signal to a suitable level for electronic processing. If these transformers have a significant air gap, the relay may be more sensitive to high-frequency components in the applied signal, regardless of measurement techniques. When the air gap is made large enough to suppress the dc offset in a fault current measurement, this high-frequency sensitivity is enhanced. Frequency compensating circuits and band-pass filters are also frequently used to reshape a distorted input signal.

High-speed static relays are not naturally filtered by the inertia of a moving mechanical assembly. Thus the speed of response may be deliberately limited to minimize false tripping due to surges and highfrequency harmonics. A static relay may also be designed for less overshoot, which can be important when the input signal is reduced to just below the pickup value.

Dual-input static relays respond to distorted waveforms depending on the design of the input transformer and the filter circuits used to condition the signals, and the design of the threshold detector which responds to either magnitude or phase angle. These options are not necessarily independent. The differences between magnitude comparison and phase-angle comparison of the derived signals are complicated. Many dual-input static relays are also high-speed relays. Their transient characteristics may be quite different than the steady-state performance.

Digital-based or computer relay systems do not necessarily avoid the harmonic problems common to most static and electromechanical relays, although there is more flexibility in processing the input 
signal and defining the specific action taken by the relay. Digital systems will have a unique response to harmonics, based on the sampling rate of the analog input signals. The transient characteristic of a computer relay system will also be a function of the algorithm used in the analog-to-digital conversion process and the frequency response of the converter. Once the signals are digitized, performance is a function of the programming built into the relay. Thus the response to distorted waveforms containing harmonics is essentially under the control of the programmer. Depending on the sophistication of the program, a more desirable or less desirable result may be attained for specific protection needs.

\subsubsection{Harmonics in Instrument Transformers}

Most forms of wave distortion and harmonics appearing on the primary systems are not suppressed by instrument transformers, and hence also appear at the input of relay circuits. In addition, further distortion and harmonics are also caused by the nonlinearity of instrument transformers [16]. These can create harmonic distortion when they are subjected to core saturation, or in high voltage applications they can create additional transients when subjected to sudden voltage changes.

Conventional current transformers (CT's) are the most likely source of wave distortion. This results primarily from CT saturation when the volt-seconds per cycle required by the burden exceeds the magnetic capability of the core. The standard accuracy classification point for a CT designed for relay applications is with a $10 \%$ error in effective RMS ratio. At this point considerable waveform distortion may be generated within the CT.

When a CT is driven into saturation by the dc component of fault current or by inrush current, even-order as well as odd-order harmonics will appear in the secondary circuit. Normally it takes time for this dc component to build up to a saturated condition. However, if the dc component is of the same polarity as the residual core flux (remanence), CT saturation can occur in less than one cycle, even with moderate values of current. Regardless of cause the generated harmonics may result in a false residual current measurement containing a larger proportion of harmonic components than the actual phase currents.

Wound type potential transformers (PT's) generally do not create noticeable harmonic distortion. However, there are two common exceptions to this rule. The first occurs when the transformers are operated above their nominal voltage rating. This condition could arise from increasing system operating voltage to improve efficiency or equipment utilization. Fortunately, many PT's are designed to operate over a wide range of applied voltage without creating distortion in the output voltage. The second exception occurs when a PT is operated with a dc offset in one winding. This can occur in the vicinity of a rectifier if designed to allow dc to flow in the ac system. While some power transformers are designed for this duty, instrument transformers generally are rot. The net result can be a significant misreading of voltage. It should be noted that magnetic PT's are subject to saturation with very low dc offsets.

Capacitive coupled voltage transformers (CCVT's) may cause additional waveform distortion because of poor transient response. Other distortion problems may result from improper burdens and internal adjustments for the intended service. These distortions may consist of harmonic or subharmonic frequencies that affect the performance primarily of high speed directional distance relays. On the other hand, CCVT's may also filter some harmonics present in the input voltage, which could actually improve performance instead of causing further distortion. 
The transmission characteristics of voltage and current transformers at harmonic frequencies are generally excellent. These devices can not be assumed to filter out any of the important harmonic components which appear on the primary terminals. Voltage transformers may transmit harmonics up to $10 \mathrm{kHz}$ with only $5 \%$ ratio error. Current transformers have better high frequency transmission characteristics than voltage transformers. CT's can transmit harmonics up to $15 \mathrm{kHz}$ with less than $1 \%$ ratio error.

\subsubsection{GIC Effects on Relay and CT Performance}

The effects of dc excitation on large power transformers is known to have profound effects on transformer and power system operation due to half-cycle saturation. The behavior of CT's in the presence of GIC is more subtle than the behavior of a large power transformer. CT's are normally limited to operation on a very small and linear segment of the saturation curve. Therefore the presence of GIC tends only to offset the normal operating point on the CT saturation characteristic.

GIC causes CT saturation in the same manner as the dc offset in a fault current, except the GIC may last for a much longer time. Both fault-induced dc offset and GIC can cause excursion in the flux level of a CT. However, in the case of GIC, the flux excursion is virtually a steady-state offset of the normal ac flux. When the GIC reaches a certain level, the combined ac and dc flux will cause the CT to operate in the nonlinear saturation region. At this point, the output becomes distorted. The lower the CT burden, or the higher the CT turns ratio, the less effect a given value of GIC will have upon the CT.

Correit relay response is first dependent upon accurate response of the CT. The variety of GICrelated relay misoperations can generally be classified into three types: (1) detection of a fault where none exists, (2) failure to detect a fault and (3) failure to detect a fault in an adequate time period. There are several classes of relaying for which misoperation may still occur due to GIC even if the CT response is accurate. In most cases, the misoperation of protective devices stems from the effects of half-cycle saturation of large power transformers. Several examples of typical protection systems that may be prone to misoperation are described in the literature [9].

There were an unusually large number of unwanted trips and some equipment damage during the March 13, 1989 geomagnetic storm. No previous magnetic storm had ever caused as many incorrect relay operations. The North American Electric Reliability Council reported 30 automatic operations during a two day period [17]. The most obvious change in the field of protective relaying is the increased use of electronic relays. Some of the electronic relays measure the peak value of the currents and are sensitive to harmonics. In the past, most of the protection schemes used electromechanical relays, which measure the effective value of currents, thus reducing sensitivity to harmonic peaks. During magnetic storms, when the harmonic content on the system increases substantially, peak-measuring relays may operate at a significantly lower effective current than electromechanical relays. A peak measuring electronic overcurrent relay triggered one major disturbance during the March 1989 storm. By increasing the settings of peak measuring relays to accommodate the higher harmonics during GIC conditions, the risk of false trips can be reduced, but this may degrade the desired protection during normal conditions.

\subsubsection{Simulation of Harmonic Effects on Undervoltage Relays}

There are a number of areas in a nuclear plant where GIC-induced harmonics could cause problems. Voltage and current measurements for protective relaying are taken from the primary distribution level all the way down to the $120 \mathrm{~V}$ level. Without an exhaustive trial and error study, it is difficult to predict which circuits and specific equipment will be affected the most by these harmonics. 
Therefore, a limited simulation study was selected which explores the response to GIC harmonics for a typical undervoltage protection scheme. The study was generic in nature and the conclusions reached should apply to various types of protective relaying. Measurement data was taken from the simulations in the form of peak values, true RMS values and average values, which covers nearly all signal input formats used by existing relays.

\subsubsection{Types of undervoltage relays}

Two types of undervoltage relays were selected for the simulation, which represent the majority of those in current use at nuclear plants. The first type is a high-speed electromechanical voltage relay, similar to GE Type NGV. It is designed to operate continuously in the pickup mode at rated voltage. The relay element consists of a telephone-type relay in series with a zener regulator connected across a full-wave rectifier. A capacitor is connected across the rectifier ac supply to protect the rectifier from severe voltage surges. A rheostat is also connected in series with the rectifier to provide pickup adjustment. The relay is intended for general applications where instantaneous detection of undervoltage conditions is required. A typical application would be undervoltage detection for emergency throw-over control equipment. Figure 6-4 is a schematic diagram of this type of relay. It is clear from this diagram and the above discussion that this relay responds to the average voltage of the input waveform, as provided by the full-wave diode rectifier.

The second type of relay chosen was a static type undervoltage relay, similar to the ABB Type ITE-27 voltage relay. Figure 6-5 shows a block diagram of the basic components in this type of relay. It is available in a high-speed instantaneous trip model or a delayed time trip version. Typical applications include undervoltage protection of motor feeder circuits and initiation of transfer in automatic bus transfer schemes. This relay is an electronic circuit consisting of a regulated reference voltage, an input signal processing circuit, a pickup circuit, a timing circuit (only for delayed-trip models), and a final output stage. There is also an optional harmonic filter circuit that interfaces with the input circuit. The input circuit is essentially a step-down transformer feeding an adjustable amplifier to provide a nominal $10 \mathrm{~V}$ peak ac voltage when the measured bus voltage is at its normal operating value. The pickup circuit is an op-amp comparator circuit which maintains the pickup (normal) state as long as the input stage continuously maintains a peak ac voltage signal of at least $10 \mathrm{~V}$. This corresponds to the normal (nontrip) condition of the system. If the input circuit peak ac voltage falls below a selectable dropout level, typically $90 \%$ of nominal voltage, the pickup circuit latches in the dropout (trip) condition until the peak ac voltage returns to at least $10 \mathrm{~V}$. If a delayed-trip signal is desired, a capacitor charging circuit is initiated from the dropout pulse to provide the time delay. This is the timing circuit referred to above.

It is clear from the above operating description that this second type of relay responds to peak values of the input signal, regardless of the fundamental value or the frequency of any harmonics present on the signal. For applications where waveform distortion is significant, a harmonic filter may be added between the input circuit and the pickup stage. The filter gain may be adjusted to maintain original voltage calibrations. The harmonic filter is an op-amp circuit consisting of three stages of second-order transfer functions, which provide a sharp low-pass function for extracting the $60 \mathrm{~Hz}$ fundamental wave.

\subsubsection{Simulation study results}

For the two types of undervoltage relays specifically considered in this study, both average values and peak values of the input voltage signal must be represented. To make the study more general RMS values will also be considered. In order to predict the effect of GIC-induced harmonics on these relays, it is not necessary to simulate the entire relay function in detail. It is only necessary to evaluate the effect 
of harmonics on the average value, the RMS value, or the peak value of the input signal, along with any frequency-dependent signal processing, such as a harmonic filter. In this way it is possible to predict possible relay errors or misoperation, by comparing these basic parameters with and without the presence of GIC-induced harmonics. The system simulations described in the previous section of the report were appropriately modified to measure the average, RMS and peak voltages at selected buses in the system, for various operating conditions with and without GIC in effect. A static undervoltage relay harmonic filter, like the one described above, was also incorporated in these simulations.

An important application of undervoltage relays in the nuclear plant model used in the previous simulations is for detecting low voltage conditions at the $4.16 \mathrm{kV}$ emergency bus feeding major motor loads, in which case it may be necessary to transfer this load to the emergency diesel units. Since the previous simulations also showed this bus to particularly sensitive to GIC-induced harmonics, it was selected to one of the key buses for the current study. The other key bus selected in this study was the $120 \mathrm{~V}$ emergency bus, which earlier studies also showed as being susceptible to significant harmonic levels when the system was subjected to GIC.

The first case considered here involved the simulated plant operating in its normal mode, with the generator supplying the main $345 \mathrm{kV}$ line and the onsite nonsafety-related loads through the normal station service transformer. Refer to Fig. 4-5 for the previous simulation model of the plant. The case considered in the present study also assumed a separate $115 \mathrm{kV}$ line supplying the safety-related (emergency) circuits through the reserve station service transformer, during normal operation of the plant. For nominal system parameters in this case, with a 50 mile $345 \mathrm{kV}$ line, a 50 mile $115 \mathrm{kV}$ line and $100 \mathrm{~A} \mathrm{GIC}$ in both lines, the frequency spectrums of the $4.16 \mathrm{kV}$ and $120 \mathrm{~V}$ emergency bus voltages are shown in Figs. 4-20 and 4-21. The corresponding voltage waveforms at these buses are shown in Figs. F23 and F-24.

This same simulation was also run for a different $115 \mathrm{kV}$ line length of 69.55 miles, which moved the line resonant frequency to the 11 th harmonic and increased the voltage THD at the emergency buses to around $12 \%$. Figures $\mathrm{H}-19$ through $\mathrm{H}-22$ show the voltage waveforms and frequency spectra for this case at the $4.16 \mathrm{kV}$ and $120 \mathrm{~V}$ emergency buses. With the GIC limited to $100 \mathrm{~A}$, this simulation was the worst case THD shown in the previous studies. The simulation was modified in the present study to include relay measurements and a harmonic filter used in the ABB Type ITE-27 static undervoltage relay. Figures 6-6 and 6-7 show the unfiltered and filtered voltages taken from the simulation for the $4.16 \mathrm{kV}$ and $120 \mathrm{~V}$ emergency buses, with $100 \mathrm{~A}$ GIC. Except for the phase shift, the filter appears to provide a good replication of the fundamental voltage waves at each of these buses. Further analysis showed that the filter was capable of removing virtually all the higher order GIC-induced harmonics except for about $20 \%$ of the second harmonic, which results in an output THD of around 1\%. Figures 6-5 and 6-6 show the same waveforms for the simulation without GIC. This verifies the filter calibration to have a fundamental voltage magnitude gain of exactly one. It also provides a measure of the fundamental voltage drop due to GIC, by comparison with the simulation results shown in Figs. 6-6 and 6-7.

Tables 6-1 and 6-2 summarize the voltage relay data taken from the above simulation, corresponding to the waveforms shown in Figs. 6-6 through 6-9. Peak values, average values and RMS values were measured from these waves for conditions with and without the nominal $100 \mathrm{~A} \mathrm{GIC}$. The percent voltage drop caused by GIC saturation is computed for each measured parameter. These voltage drops are then compared to determine possible relay errors in reading the input voltage wave. For the peak voltage measurement both filtered and unfiltered values were compared, and since the filter provides an accurate peak value for the fundamental, the error represents a false high reading with no filter. This means in Table 6-1 that if an adequate filter is not used, the relay would fail to trip unless 
the fundamental $4.16 \mathrm{kV}$ bus voltage fell to $3.57 \%$ lower than the relay setting. Similarly, in Table 6-2 an undervoltage relay would fail to trip unless the fundamental $120 \mathrm{~V}$ bus voltage fell to $11.5 \%$ lower than the relay setting.

The average voltage dat 1 compares the true average measured from the distorted wave to the computed average from the fundamental sine wave. The computed average value is derived from the filtered peak value, which is assumed to be an accurate measurement of the fundamental sine wave. The relay should respond to the ideal computed average, but actually responds to the measured (true) average, which contains harmonics. The error represents a percent difference in voltage drops due to GIC, which indicates a false high reading for the actual distorted waveform. The RMS viltage data compares the true RMS measured from the distorted wave to the computed RMS from the fi ndamental sine wave. The computed RMS value is again derived from the filtered peak value. The relay should respond to the ideal computed RMS, but actually responds to the measured (true) RMS, which contains harmonics. The error represents a percent difference in voltage drops due to GIC, which again indicates a false high reading for the actual distorted waveform. Tables 6-1 and 6-2 show that both average value errors and RMS value errors are quite small, compared to potential peak value errors.

A variation of the above case study was also simulated for a 50 mile $115 \mathrm{kV}$ line, with the GIC increased to $150 \mathrm{~A}$. This is still well within the maximum GIC observed in previous geomagnetic disturbances. In this case the new line resonance shifts to 16th harmonic, and the THD level increases to over $19 \%$ for the voltages at the $4.16 \mathrm{kV}$ emergency bus and to almost $16 \%$ for the $120 \mathrm{~V}$ emergency bus voltage. Figures $6-10$ through 6-13 show the corresponding voltage waveforms and frequency spectra for this case. Note the very pronounced distortion in these waveforms and that there is again a wide range of significant harmonics present. This is a worse case than the earlier study. Tables 6-3 and 6-4 summarize the relay data taken from the simulation for this case, corresponding to the waves in Figs. 6-10 and 6-12. With the higher harmonic levels, the unfiltered peak voltage measurements showed significant increase in error, which was over $19 \%$ at both the $4.16 \mathrm{kV}$ and $120 \mathrm{~V}$ emergency bises. This corresponds approximately to the THD level at these buses. This means if the static relay (without harmonic filter) was set to trip at a typical undervoltage setting of $90 \%$, the fundamental would actually have reach about $70 \%$ of its normal level before the relay would trip. From Tables $6-3$ and $6-4$ it is clear that relays responding to the average voltage measurement would experience only minor errors, even with the significantly increased harmunic distortion. Note that measured average voltage level is lower in this case, because of resonance shift to this even 16th harmonic. A similar statement can be made for the RMS values observed, although there is a moderate increase in percent error for this case.

The final simulated case study performed involved the system in the startup mode of operation. As in the earlier simulation, it was assumed that the normal station service transformer is disconnected from the normal onsite distribution system. Instead, the reserve station service transformers are assumed to carry the entire onsite load, including the nonsafety-related and the emergency system loads. Therefore, the only source of harmonics in the plant are from a GIC flow through the grounded neutrals of the reserve station service transformers. Even if GIC flows through the step-up transformer there is no path for harmonics to enter the station. Referring to the simulation model in Fig. 4-5, this study was performed by opening the breakers from the normal station service transformer $T 2$ to the $13.8 \mathrm{kV}$ buses $\mathrm{B} 001$ and $\mathrm{B} 003$, then closing the NO breaker from the reserve station service transformer T3 to bis B003. As before, a $0.15 \mathrm{pu}$ source reactance was placed in series with the remote voltage source at the far end of the $345 \mathrm{kV}$ line. Figures 4-23 and 4-24 show the frequency spectrums for voltages at the 4.16 $\mathrm{kV}$ and the $120 \mathrm{~V}$ emergency buses, with nominal system parameters during the startup mode, which include a 50 mile $345 \mathrm{kV}$ line and a GIC level of $50 \mathrm{~A}$. The corresponding voltage waveforms are shown in Figs. F-26 and F-28. 
The above startup case study was modified to include a 42.38 mile transmission line, which changed the line resonance from the 10th harmonic to the 11th harmonic, with an increase in voltage THD at both the $4.16 \mathrm{kV}$ and $120 \mathrm{~V}$ emergency buses. The corresponding voltage waveforms and frequency spectra are shown in Figs. H-25 through H-28. Tables 6-5 and 6-6 summarize the relay data taken from the simulation for this case, corresponding to the waves in Figs. H-25 and H-27. With these more moderate harmonic levels, the unfiltered peak voltage measurements showed somewhat moderate error, which was under $7 \%$ at the $4.16 \mathrm{kV}$ emergency bus and about $4.5 \%$ at the $120 \mathrm{~V}$ bus. This again corresponds approximately to the THD level at these buses. From Tables 6.5 and 6.6 it may also be observed that undervoltage relays responding to an average voltage or an RMS voltage measurement would experience only minor errors.

\subsection{Conclusions}

This study focussed on the effects of harmonics on the uninterruptible Power Supply (UPS) system and protective relaying used in the in-plant electrical distribution system. Based on this study, the following conclusions can be supported:

1. The UPS system may be considered nearly immune to the effects of high harmonic levels present in the plant distribution system by virtue of the design and the configuration in which they are used in nuclear plants.

2. Likewise, the $120 \mathrm{~V} \mathrm{AC}$ vital instrumentation busses which served UPS system will experience insignificant effects due to the high harmonics levels present in the plant distribution system.

3. It is difficult to generalize on the effects of harmonics on protective relaying in a power plant. Relay manufacturers have introduced a wide variety of relay designs, which use different principles of operation. Some respond to average or RMS values; others respond to peak values. Some provide inherent filtering, others offer optional filters. Most relays are designed to be calibrated and tested using a fundamental frequency sine wave, so it is difficult to determine how a specific type will respond to distorted waves containing harmonics. This diversity in designs and lack of information has contributed to the void in IEEE standards on relays operating with harmonic distortion. Hopefully, new standards will emerge as more information becomes available to the industry and the effects of harmonics can be more generalized.

This study has focused on the effect of GIC-induced harmonics on undervoltage relays in a typical nuclear plant. A specific application considered was the detection of low voltage conditions at the 4.16 $\mathrm{kV}$ emergency bus feeding major motor loads, in order to transfer this load to the emergency diesel units. Simulation studies showed under various conditions that both electromechanical and static type relays tended to give a false high reading due to harmonics. This means that the fundamental frequency voltage would have to drop further than the protective setting in order to trip the relay. This may dictate a higher trip voltage setting, which could lead to unwanted early tripping when harmonics are not present. For relays that respond to average values or RMS values the errors were quite small (1\%-2\%), but for relays that respond to peak values the errors can be very large (19\%) if a harmonic filter is not used. With an effective harmonic filter, peak voltage relaying error can drop to about $1 \%$ or less. Generally speaking, a good harmonic filter is highly recommended for all relays which must operate in plants subject to GIC. 
Table 6-1. Comparison of Relay Data at $4.16 \mathrm{kV}$ Emergency Bus During Normal Operation (100 A GIC) with Separate $69.55 \mathrm{mi} 115 \mathrm{kV}$ Line.

\begin{tabular}{|c|c|c|c|c|c|c|}
\hline \multirow{2}{*}{$\begin{array}{l}\text { Per phase } \\
\text { Quantities }\end{array}$} & \multicolumn{2}{|c|}{ Peak Volts } & \multicolumn{2}{|c|}{ Average Volts } & \multicolumn{2}{|c|}{ RMS Volts } \\
\hline & Unfiltered & Filtered & Measured & $2($ peak V) $/ \pi$ & Measured & $($ Peak V) $N 2$ \\
\hline w/o GIC & 3357 & 3358 & 2137 & 2138 & 2374 & 2374 \\
\hline with GIC & 3315 & 3196 & 2038 & 2035 & 2293 & 2260 \\
\hline \% Drop & 1.25 & 4.82 & 4.63 & 4.82 & 3.41 & 4.80 \\
\hline$\%$ Error & \multicolumn{2}{|c|}{3.57} & \multicolumn{2}{|c|}{0.19} & \multicolumn{2}{|c|}{1.39} \\
\hline
\end{tabular}

Table 6-2. Comparison of Relay Data at 120 V Emergency Bus During Normal Operation (100 A ( ) ) with Separate $69.55 \mathrm{mi} 115 \mathrm{kV}$ Line.

\begin{tabular}{||l|c|c|c|c|c|c||}
\hline \hline \multirow{2}{*}{$\begin{array}{l}\text { Per phase } \\
\text { Quantities }\end{array}$} & \multicolumn{2}{|c|}{ Peak Volts } & \multicolumn{2}{c|}{ Average Volts } & \multicolumn{2}{c||}{ RMS Volts } \\
\cline { 2 - 7 } & Unfiltered & Filtered & Measured & 2(peak V)/ & Measured & (Peak V) $/ 2$ \\
\hline w/o GIC & 163.7 & 163.7 & 104.2 & 104.2 & 115.7 & 115.7 \\
\hline with GIC & 174.7 & 155.9 & 101.1 & 99.2 & 112.2 & 110.2 \\
\hline \% Drop & -6.72 & 4.76 & 2.98 & 4.80 & 3.02 & 4.75 \\
\hline \% Error & \multicolumn{2}{|c|}{11.5} & \multicolumn{3}{c|}{1.82} & \multicolumn{2}{c|}{1.73} \\
\hline
\end{tabular}

Table 6-3. Comparison of Relay Data at $4.16 \mathrm{kV}$ Emergency Bus During Normal Operation (150 A GIC) with Separate $50 \mathrm{mi} 115 \mathrm{kV}$ Line.

\begin{tabular}{|c|c|c|c|c|c|c|}
\hline \multirow{2}{*}{$\begin{array}{l}\text { Per phase } \\
\text { Quantities }\end{array}$} & \multicolumn{2}{|c|}{ Peak Volts } & \multicolumn{2}{|c|}{ Average Volts } & \multicolumn{2}{|c|}{ RMS Volts } \\
\hline & Unfiltered & Filtered & Measured & $2($ peak V) $/ \pi$ & Measured & $($ Peak V) $/ 2$ \\
\hline w/o GIC & 3357 & 3357 & 2137 & 2137 & 2373 & 2374 \\
\hline with GIC & 3847 & 3186 & 2058 & 2028 & 2311 & 2253 \\
\hline \% Drop & -14.6 & 5.09 & 3.70 & 5.10 & 2.61 & 5.10 \\
\hline$\%$ Error & \multicolumn{2}{|c|}{19.7} & \multicolumn{2}{|c|}{1.40} & \multicolumn{2}{|c|}{2.49} \\
\hline
\end{tabular}


Table 6-4. Comparison of Relay Data at 120 V Emergency Bus During Normal Operation (150 A GIC) with Separate $50 \mathrm{mi} 115 \mathrm{kV}$ Line.

\begin{tabular}{||l|c|c|c|c|c|c||}
\hline \multirow{2}{*}{$\begin{array}{l}\text { Per phase } \\
\text { Quantities }\end{array}$} & \multicolumn{2}{|c|}{ Peak Volts } & \multicolumn{2}{c|}{ Average Volts } & \multicolumn{2}{c|}{ RMS Volts } \\
\cline { 2 - 8 } & Unfiltered & Filtered & Measured & 2(peak V)/ & Measured & (Peak V) $/ 2$ \\
\hline w/o GIC & 163.6 & 163.6 & 104.1 & 104.2 & 115.7 & 115.7 \\
\hline with GIC & 186.6 & 155.4 & 100.2 & 98.9 & 112.4 & 109.9 \\
\hline$\%$ Drop & -14.06 & 5.01 & 3.75 & 5.09 & 2.85 & 5.01 \\
\hline \% Error & \multicolumn{2}{|c|}{19.1} & \multicolumn{3}{c|}{1.34} & \multicolumn{2}{c|}{2.16} \\
\hline
\end{tabular}

Table 6-5. Comparison of Relay Data at $4.16 \mathrm{kV}$ Emergency Bus During Startup Mode (50 A GIC) with $42.38 \mathrm{mi} 345 \mathrm{kV}$ Line.

\begin{tabular}{|c|c|c|c|c|c|c|}
\hline \multirow{2}{*}{$\begin{array}{l}\text { Per phase } \\
\text { Quantities }\end{array}$} & \multicolumn{2}{|c|}{ Peak Volts } & \multicolumn{2}{|c|}{ Average Volts } & \multicolumn{2}{|c|}{ RMS Volts } \\
\hline & Unfiltered & Filtered & Measured & $2($ peak V) $/ \pi$ & Measured & $($ Peak V) $/ / 2$ \\
\hline w/o GIC & 3363 & 3361 & 2139 & 2140 & 2376 & 2377 \\
\hline with GIC & 3538 & 3307 & 2090 & 2105 & 2336 & 2338 \\
\hline \% Drop & -5.20 & 1.61 & 2.29 & 1.64 & 1.68 & 1.64 \\
\hline$\%$ Error & \multicolumn{2}{|c|}{6.81} & \multicolumn{2}{|c|}{0.65} & \multicolumn{2}{|c|}{0.04} \\
\hline
\end{tabular}

Table 6-6. Comparison of Relay Data at 120 V Emergency Bus During Startup Mode (50 A GIC) with $42.38 \mathrm{mi} 345 \mathrm{kV}$ Line.

\begin{tabular}{||l|c|c|c|c|c|c||}
\hline \multirow{2}{*}{$\begin{array}{l}\text { Per phase } \\
\text { Quantities }\end{array}$} & \multicolumn{2}{|c|}{ Peak Volts } & \multicolumn{2}{c|}{ Average Volts } & \multicolumn{2}{c||}{ RMS Volts } \\
\cline { 2 - 7 } & Unfiltered & Filtered & Measured & 2(peak V)/ & Measured & (Peak V) $/ 2$ \\
\hline w/o GIC & 163.3 & 163.7 & 104.2 & 104.2 & 115.7 & 115.8 \\
\hline with GIC & 167.3 & 160.3 & 101.9 & 102.1 & 113.7 & 113.3 \\
\hline$\%$ Drop & -2.45 & 2.08 & 2.21 & 2.02 & 1.73 & 2.16 \\
\hline$\%$ Error & \multicolumn{2}{|c|}{4.53} & \multicolumn{2}{c|}{0.19} & \multicolumn{2}{c|}{0.43} \\
\hline
\end{tabular}




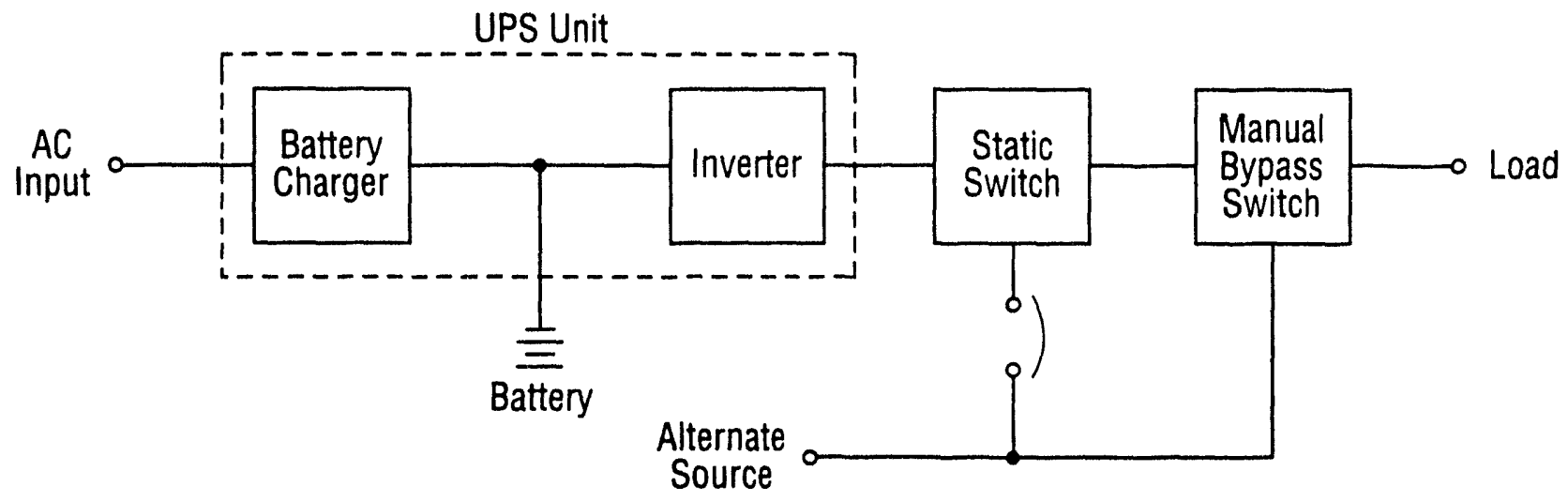

Fig. 6.1 UPS in float type configuration

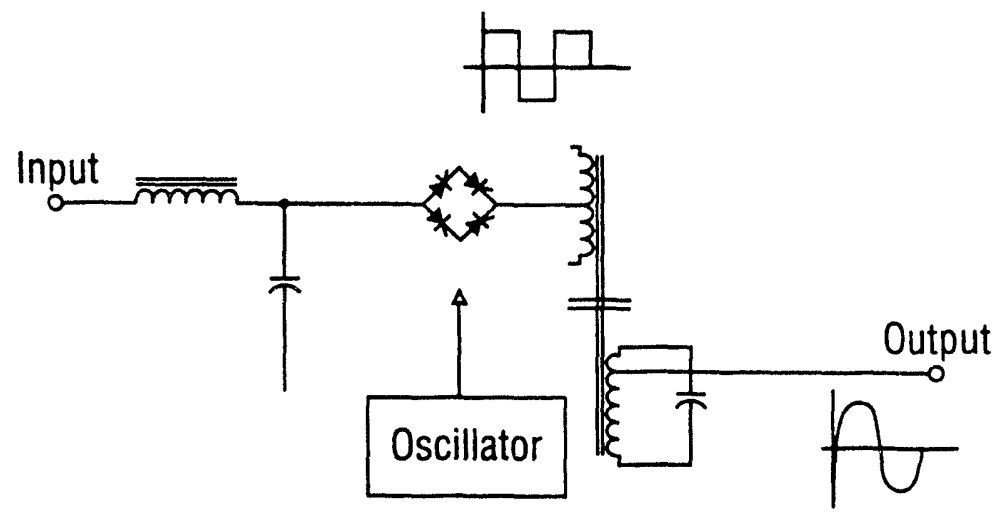

Fig. 6.2 Ferro-resonant transformer inverter

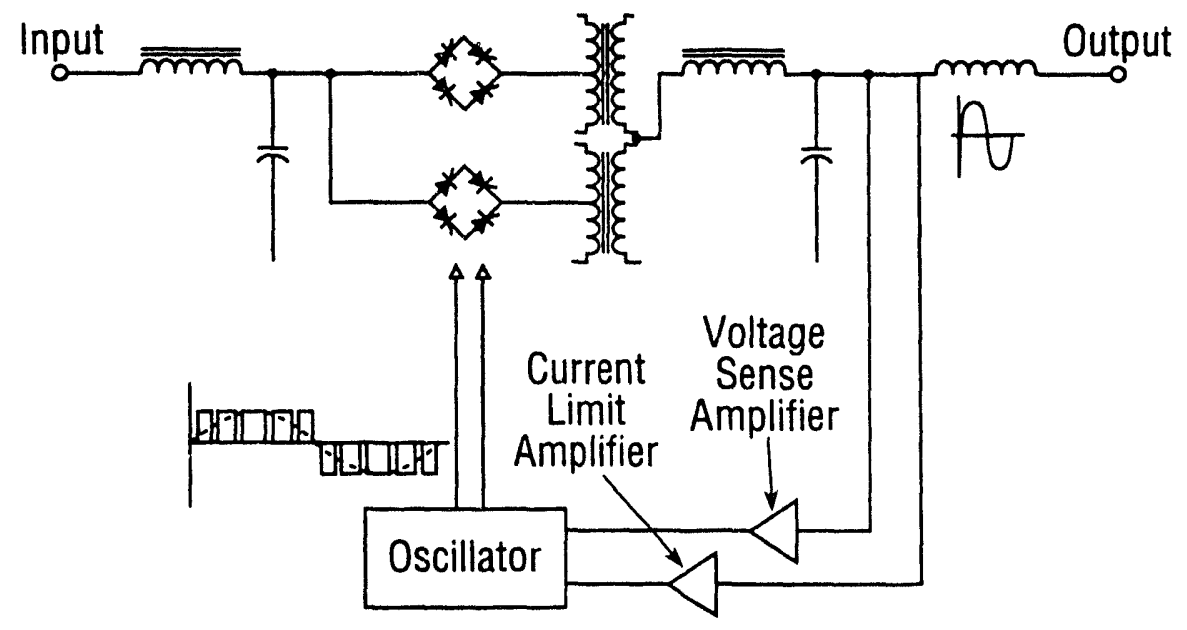

Fig. 6.3 Pulse width modulated inverter 


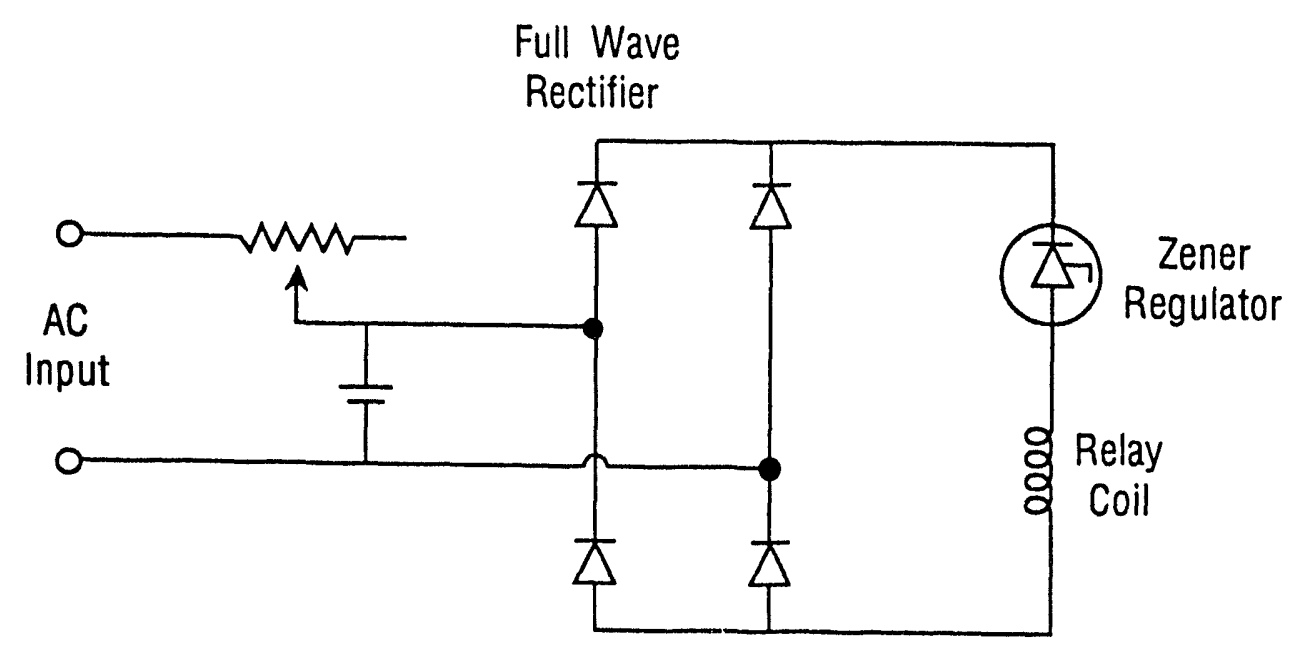

Fig. 6.4 Basic circuit of GE Type NGV undervoltage relay.

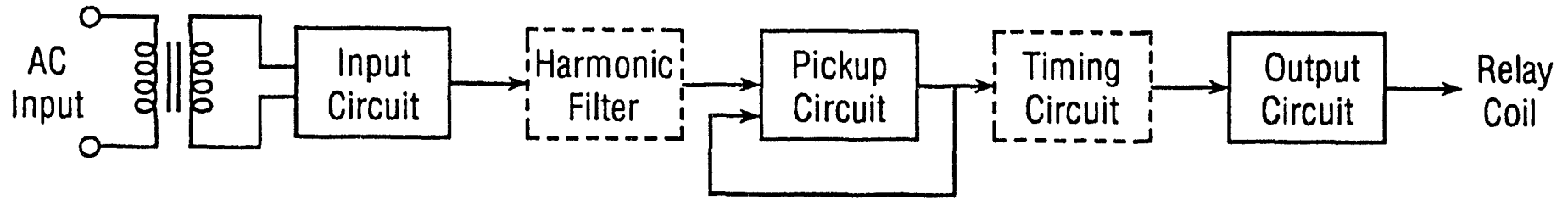

Fig. 6.5 Block diagram of ABB Type ITE-27 undervoltage relay. 


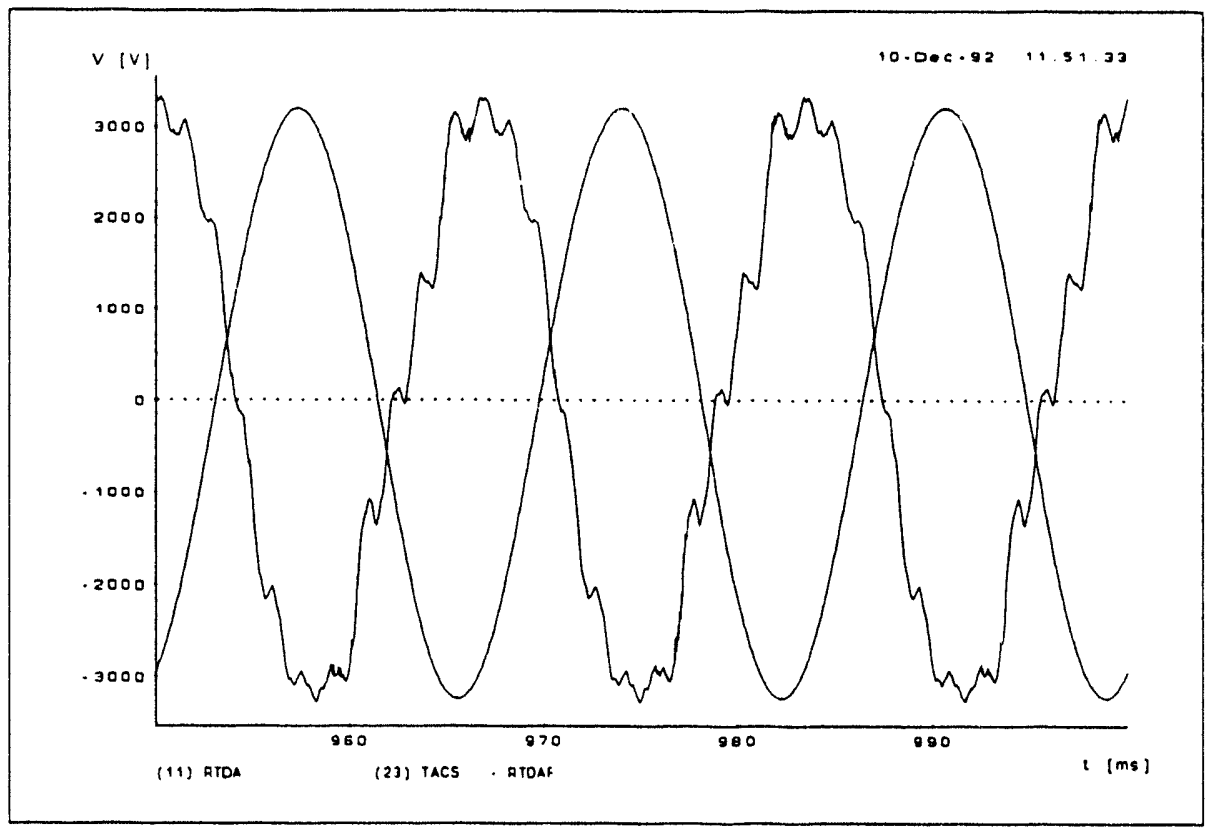

Fig. 6.6 Filtered and unfiltered voltages at $4.16 \mathrm{kV}$ side of reserve station service transformer during normal operation (100 A GIC) with separate $69.55 \mathrm{mi} 115 \mathrm{kV}$ line.

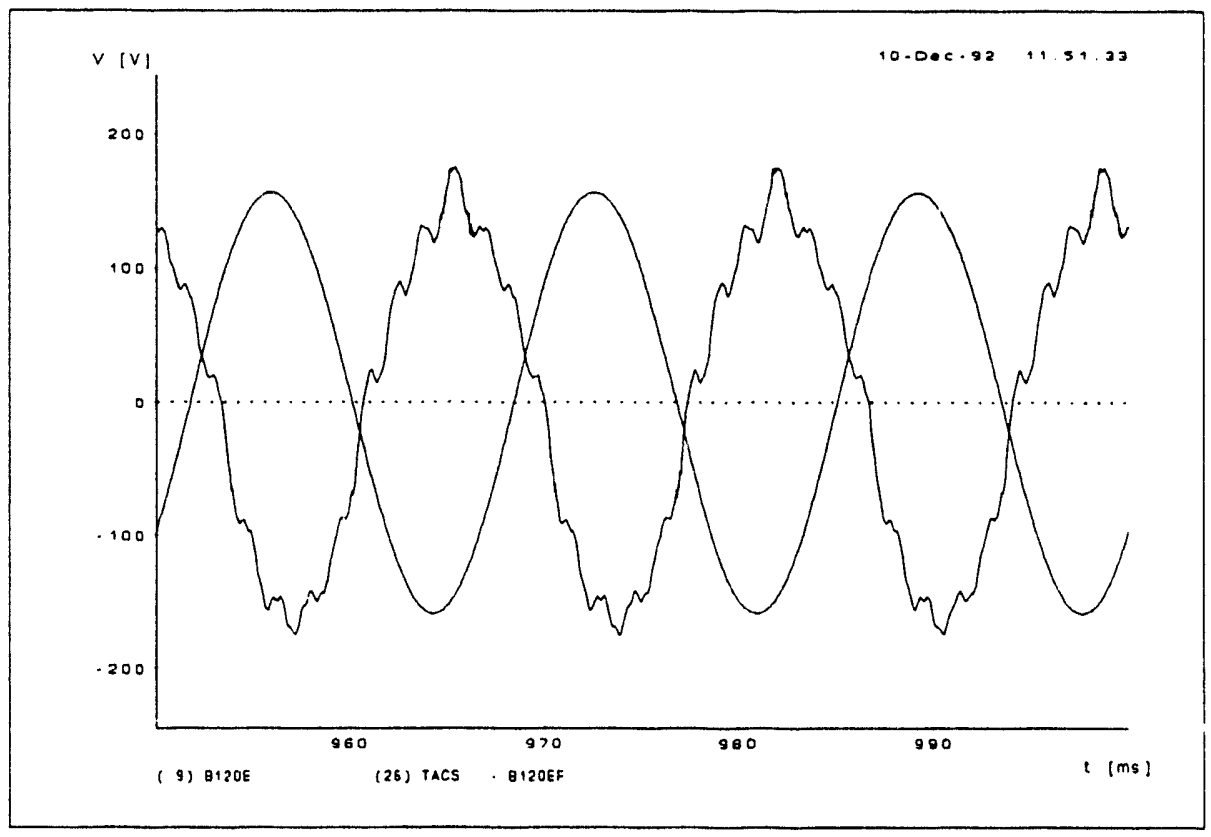

Fig. 6.7 Filtered and unfiltered voltages at $120 \mathrm{~V}$ emergency system bus during normal operation (100 A GIC) with separate $69.55 \mathrm{mi} 115 \mathrm{kV}$ line. 

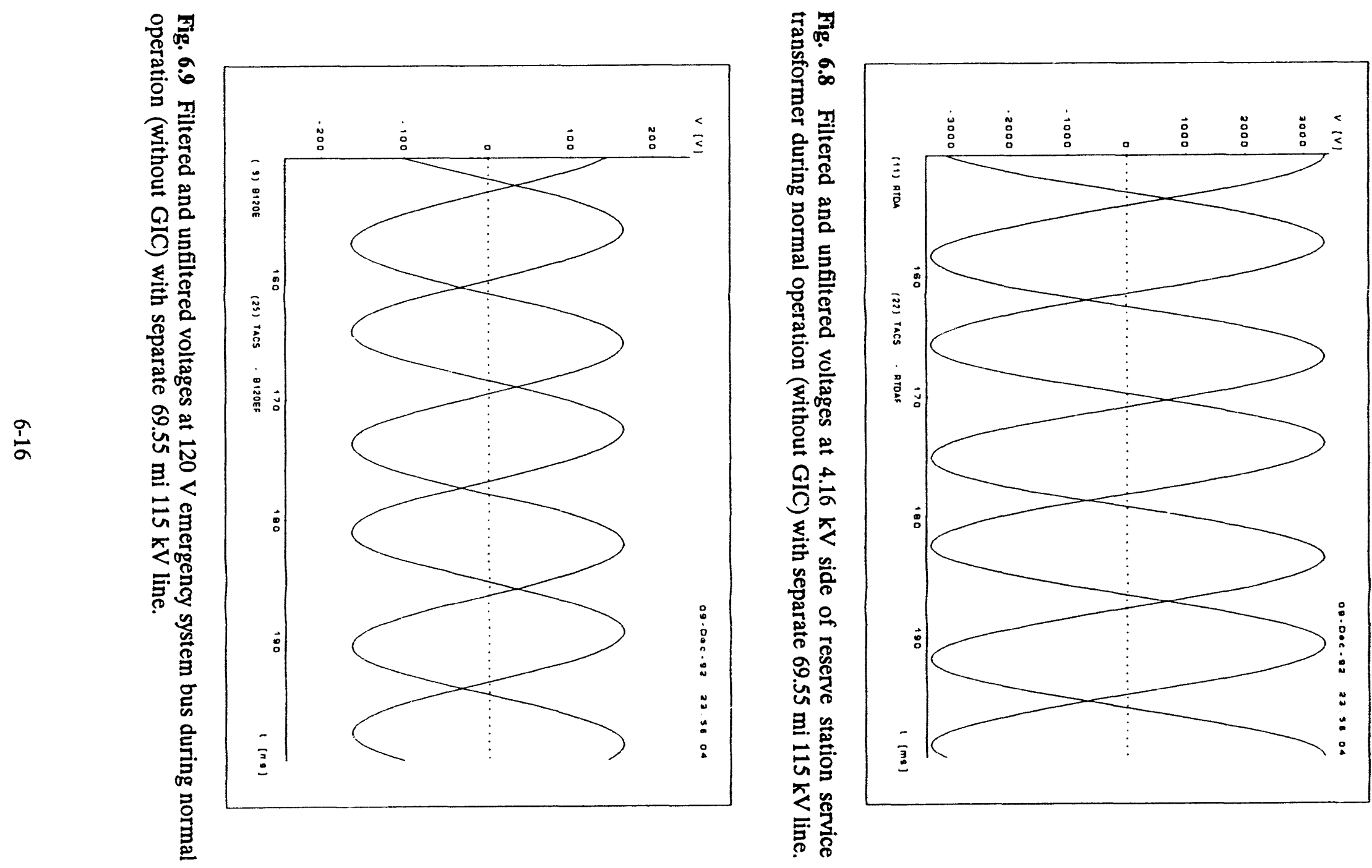


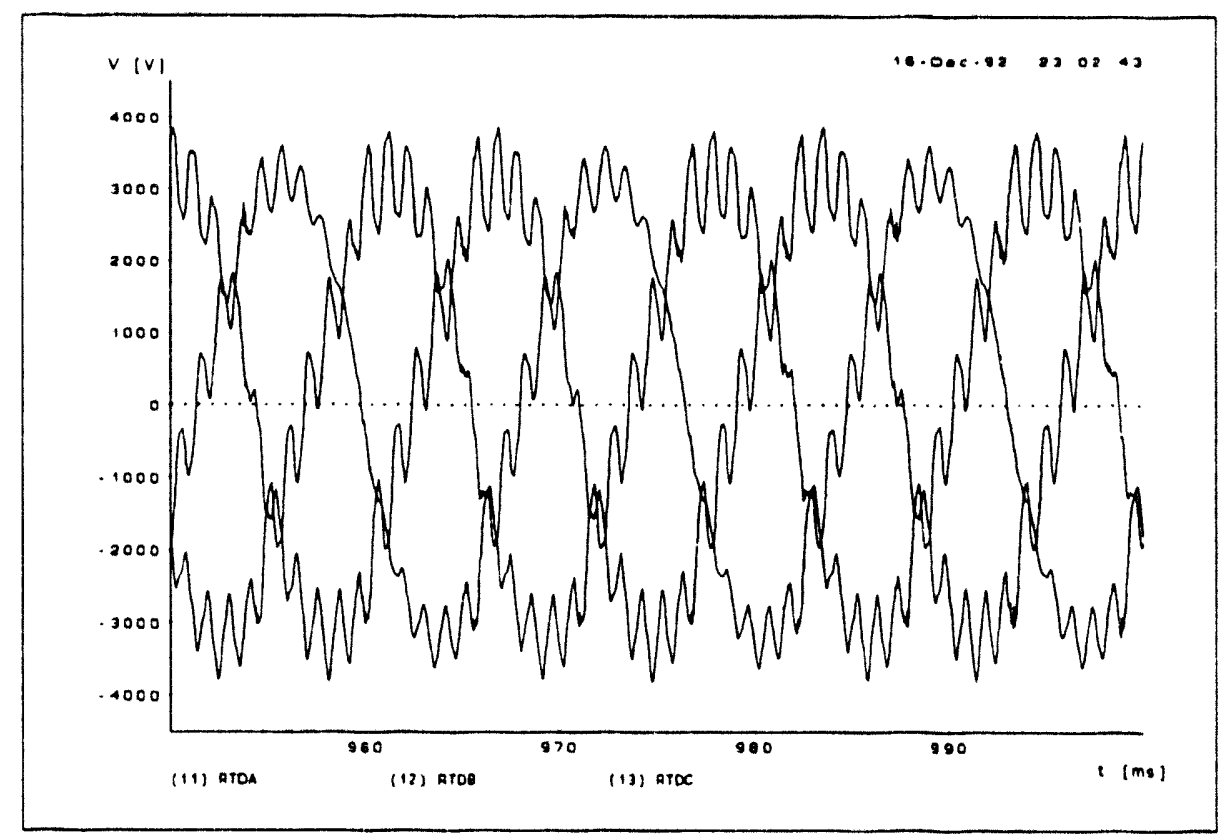

Fig. 6.10 Voltages on $4.16 \mathrm{kV}$ side of reserve station service transformer during normal operation (150 A GIC) with separate $50 \mathrm{mi} 115 \mathrm{kV}$ line.

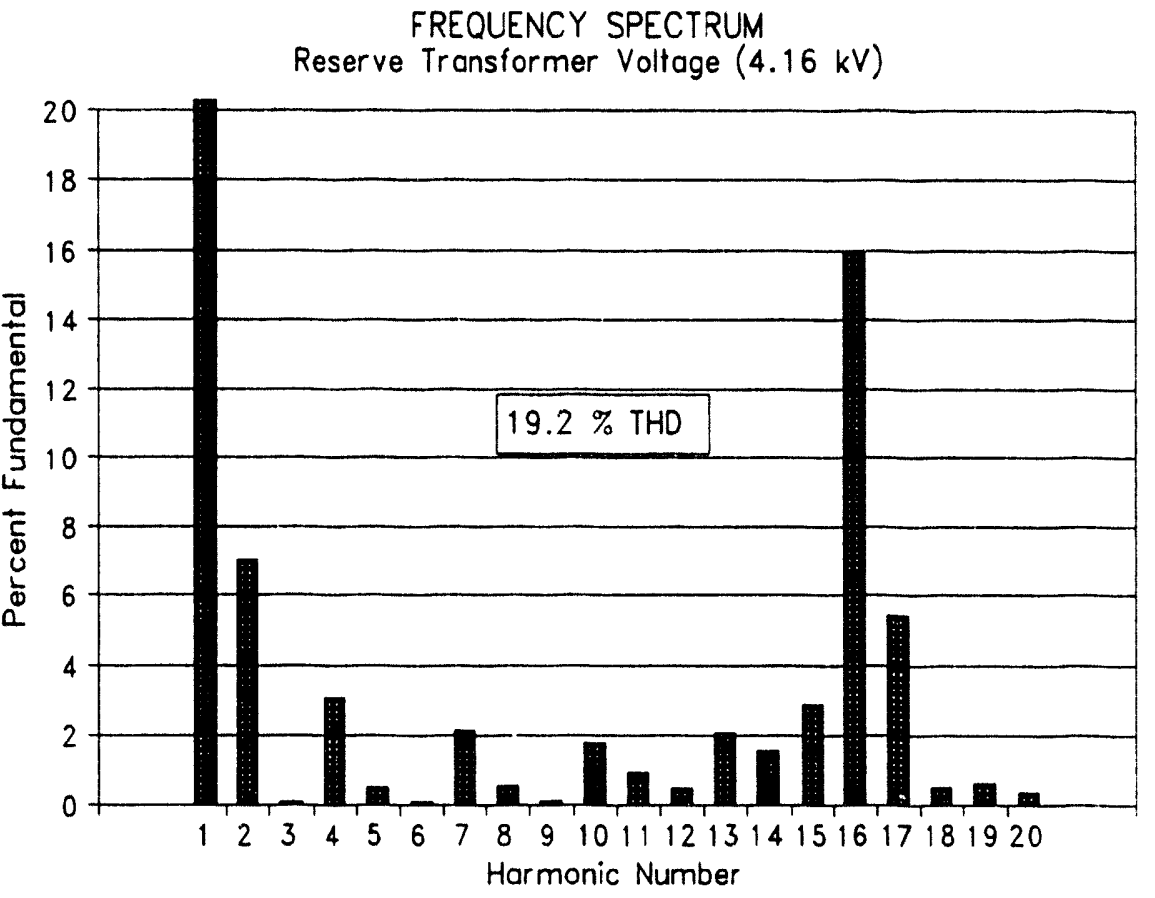

Fig. 6.11 Frequency spectrum of phase $A$ voltage on $4.16 \mathrm{kV}$ side of reserve station service transformer during normal operation (150 A GIC) with separate $50 \mathrm{mi} 115 \mathrm{kV}$ line. 


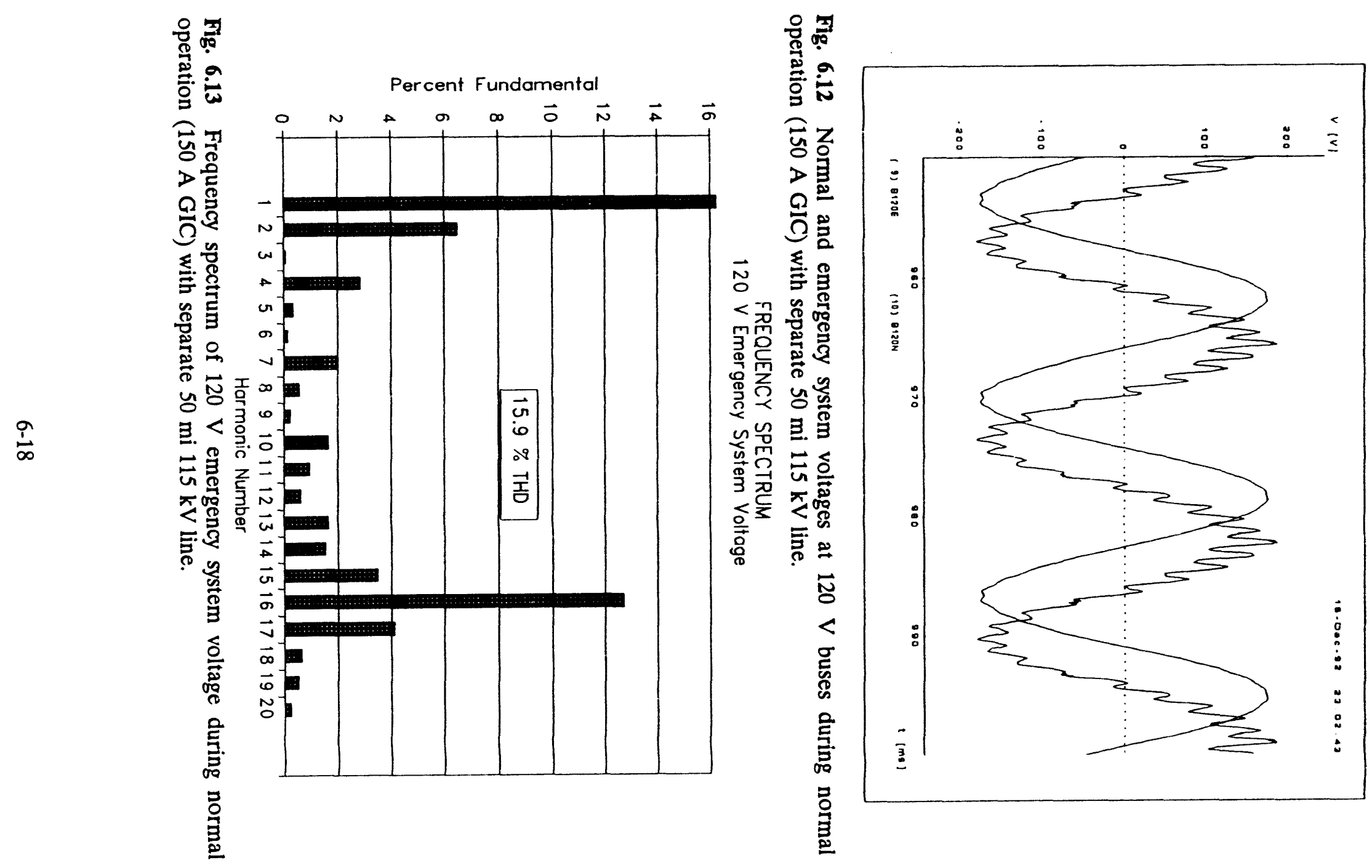




\section{EFFECT OF TRANSIENT CONDITIONS ON GIC-INDUCED HARMONICS}

Most of this study of GIC-induced harmonics has focussed attention on steady-state phenomena. It may also be possible that transient conditions combined with GIC pheiromena can produce even greater harmonic effects than either could produce by itself. Such conditions are probably rare, but should nevertheless be considered briefly. This problem is further complicated by the fact that dc offsets in the transformer magnetizing currents can last a relatively long period of time, both those caused by long-term GIC as well as those caused by other shorter-term transients. Therefore, it may be difficult to distinguish between strictly GIC produced harmonics and possible combinations of GIC and other more routinely occurring transients. Also, the transient may not be important in itself, but may lead to a new steady-state operating condition with entirely different, perhaps worse, harmonic levels. To further complicate matters, the GIC may take two minutes or more to fully saturate the GSU transformer; while other transformers may saturate sooner.

\subsection{Modified Simulation Studies}

In order to investigate the possibility of compounding harmonics due to system transients occurring during GIC events, the EMTP simulation of the plant distribution system was modiuud to include transient conditions that appeared likely to increase harmonic levels. There are two basic conditions that need to be considered. First, closing in a major load can produce large transient currents with dc offsets, which tend to further increase and unbalance the saturated transformer magnetizing currents during a GIC event. This, in turn, can promote higher harmonic levels in the underlying distribution network, although the effect is temporary but relatively long lasting, perhaps minutes. The second basic condition considered is the tripping of a major load downstream from the GIC-affected transformer. Although, this in itself only slightly increases magnetizing currents due to rising voltages, more importantly it increases the effective load impedance seen by the transformer, which can dramatically raise the harmonic levels in the downstream buses. This was observed in previous steadystate simulations. Eventually, this type of transient condition leads to a possibly worse new steady-state operating point for the system, as long as GIC remains in effect.

The first case considered in the transient simulation studies involved the station operating in the start-up mode. This mode of operation was similar to that described earlier for the steady-state studies in the start-up mode. As before, it was assumed that the normal station service transformer is disconnected from the normal onsite distribution system, and the reserve station service transformers are assumed to carry the entire onsite load, including the nonsafety-related and the emergency system loads. Referring to the simulation model in Fig. 4-5, this study was performed by opening the breakers from the normal station service transformer $\mathrm{T} 2$ to the $13.8 \mathrm{kV}$ buses $\mathrm{B} 001$ and $\mathrm{B} 003$, then closing the NO breaker from the reserve station service transformer $\mathrm{T} 3$ to bus B003. Again a 0.15 pu source reactance was placed in series with the remote voltage source at the far end of the 42.38 mile $345 \mathrm{kV}$ line. The line resonance is approximately at the 11 th harmonic. It is important to note in this assumed start-up mode that the reserve station service transformur T3 feeds only bus B003, and that one of the $12000 \mathrm{Hp}$ motor loads represents a major part of the total load on the bus. For this reason the closing in and the tripping of this type of motor was selected for transient study, since it seemed likely to have some effect on GICinduced harmonics.

\subsection{Simulation Results}

Figures 7-1 through 7-7 show sample waveforms from the transient simulation of the closing in of one $12000 \mathrm{Hp}$ motor load during the start-up mode of operation with $50 \mathrm{~A} \mathrm{GIC}$. Figure 7-1 shows the 
transient magnetizing currents in the reserve station service transformer, following a previous steady-state level of about $3800 \mathrm{~A}$ peak. When the motor is closed in, the transformer magnetizing currents become unbalanced, but the peak saturation level is increased on only one phase. However, the unbalanced magnetizing currents persist well beyond the motor transient overcurrents and dc offsets shown in Fig. 7-2. Even with this long lasting magnetizing current transient, the moderate increase in magnitude on one phase should not by itself generate a significant increase in harmonic level. In fact, this effect on magnetizing current is more than compensated by the decrease in effective load impedance by the closing in of the motor, which tends to decrease harmonic levels.

Figure 7-3 shows the $4.16 \mathrm{kV}$ voltages at the reserve station service transformer, in steady-state before the motor is closed in. Substantial levels of 11th and other harmonics are readily apparent on these waveforms. The levels are much higher than in the previous steady-state studies, where the motor was assumed to be already connected. Compare Fig. 7-3 with Fig. F-26 and H-25. The increased harmonics in this case are due primarily to the higher effective load impedance with motor not connected, since the magnetizing currents are about the same with or without the motor on line. Figure $7-4$ shows the transient A-phase voltage at the $4.16 \mathrm{kV}$ reserve station transformer bus immediately following motor close-in, and Fig. 7-5 shows all three phases after about 0.3 seconds. It is clear from these waveforms that harmonic conditions have not worsened in this case, even though the motor start-up transients have unbalanced and increased one phase of the transformer magnetizing currents. This shows that the decrease in effective load impedance when closing in the motor is more important than the transient effect on magnetizing current. Figures 7-6 and 7-7 show a similar before and after effect for the voltages at the normal and emergency $120 \mathrm{~V}$ buses. The same conclusions can again be reached for these lower voltage waveforms.

Figures 7-8 through 7-10 show transient waveforms for the start-up mode of operation when the $12000 \mathrm{Hp}$ motor load is tripped. Figure 7-8 shows the transient magnetizing currents in the reserve station service transformer. Here the effect is rather insignificant, because there are no large starting currents or dc offsets to induce changes in the magnetizing peaks. The slight overall increase may be attributed to the rise in voltages due to the unloaded system. Figures 7-9 and 7-10 show the $4.16 \mathrm{kV}$ voltages at the reserve station service transformer, before and 0.3 seconds after the motor tripping. The substantial increase in harmonics cannot be due to the slight increase in magnetizing currents, but again must be due to the increase in effective load impedance when the motor is tripped.

Other transient cases were also considered, but none produced any worst-case harmonic levels, as compared to predictions from steady-state simulations. Closing in and tripping of large motor loads was also simulated for the normal (non-start-up) mode of plant operation with GIC in effect, but the effect on harmonic levels was quite small. This might be expected, because a $12000 \mathrm{Hp}$ motor is a much smaller proportion of the total station load under normal operating conditions. Thus, a small change in load impedance will produce only minor changes in harmonics. The final closing in of the $345 \mathrm{kV}$ transmission line during the start-up phase was also simulated, with GIC beginning at the time of closing. This case produced significant initial transients, but the induced transformer magnetizing currents during the long magnetic transient period were never worse than the final steady-state saturation levels. Harmonic levels were correspondingly worst after final steady-state conditions were reached.

\subsection{Conclusions for Transient Conditions}

There are two basic transient conditions in a plant which can affect GIC-induced harmonics: (1) closing in a major load, and (2) tripping a major load. The first condition can result in large overcurrents and dc offsets, which can produce temporary but long lasting transients in the transformer magnetizing 
currents. Although transient increase in saturation tends to increase harmonics, this effect appears to be dominated by the decrease in effective load impedance after close-in, so the net effect is actually a reduction in harmonic level. The second condition is not associated with overcurrents, unless a fault is involved, thus it may not produce large changes in transformer saturation. However, tripping eventually results in an increase in effective load impedance, which tends to increase voltage harmonics even if transformer magnetizing currents do not change significantly. It appears that the transient condition itself does not cause higher harmonics, but can lead to new steady-state conditions which do cause higher harmonics. 


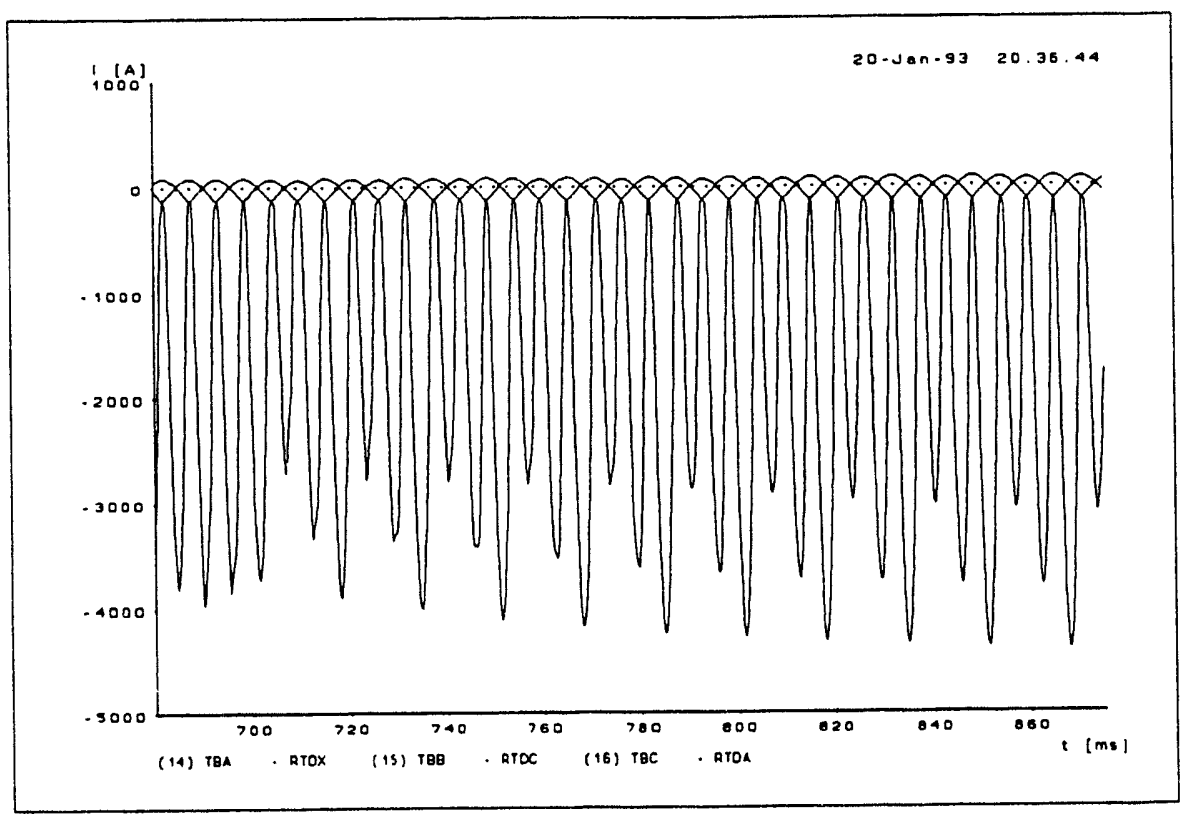

Fig. 7-1. Reserve station service transformer magnetizing currents immediately after closing in $12000 \mathrm{Hp}$ motor load during start-up mode, with $50 \mathrm{~A} \mathrm{GIC,}, 42.38 \mathrm{mi}$ line and remote source impedance.

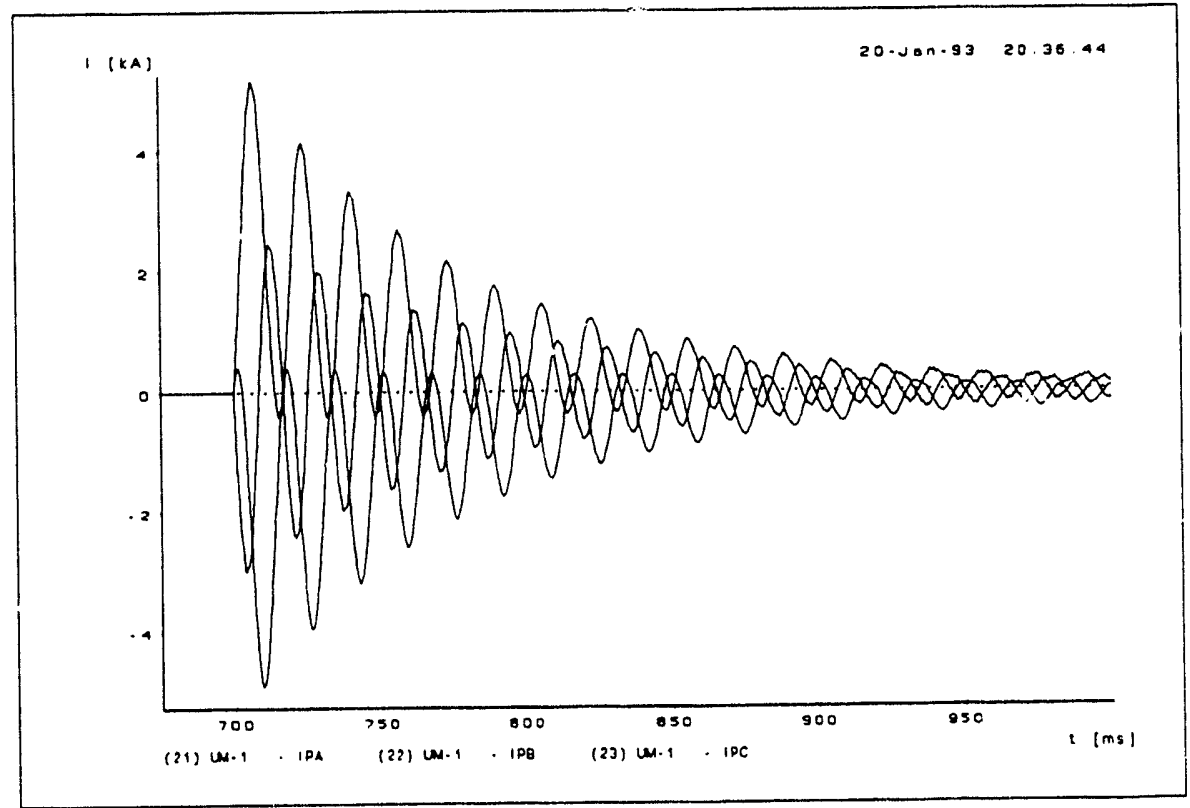

Fig. 7-2. Transient motor currents after closing in $12000 \mathrm{Hp}$ motor load during start-up mode, with $50 \mathrm{~A} \mathrm{GIC}, 42.38 \mathrm{mi}$ line and remote source impedance. 


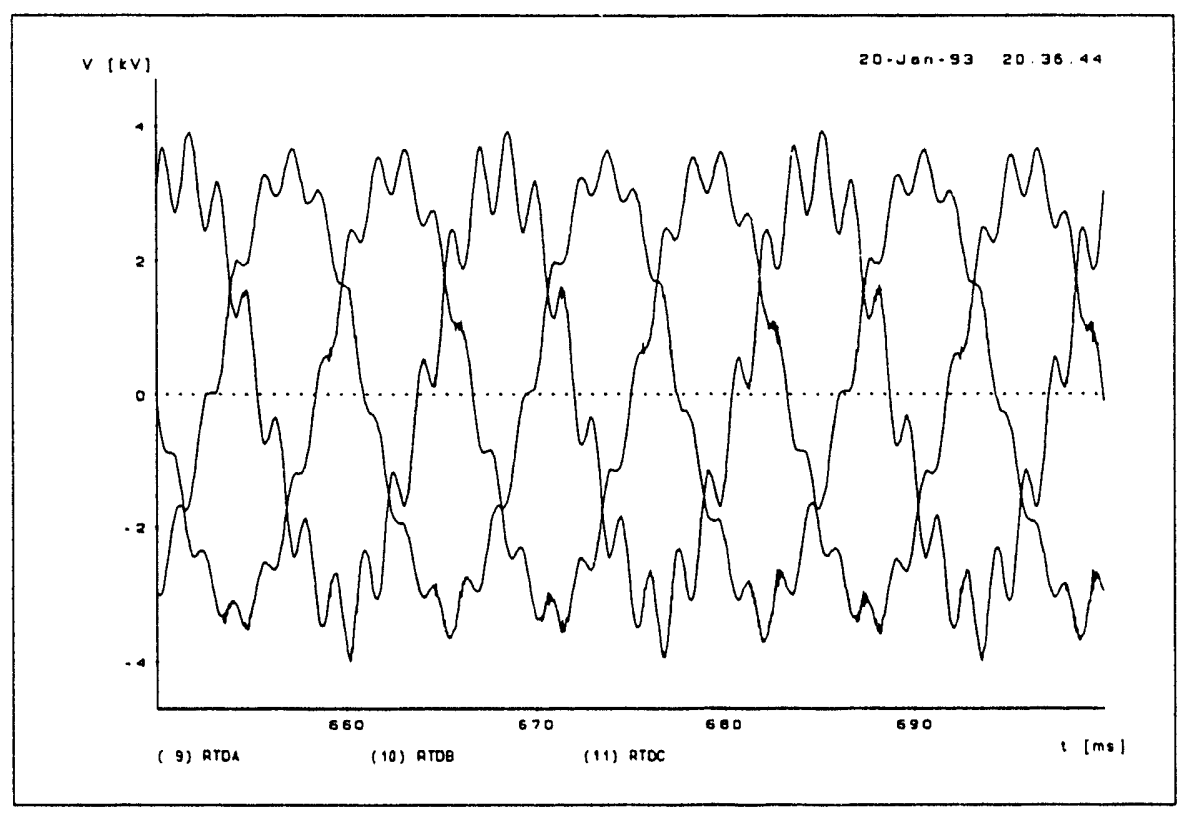

Fig. 7-3. Reserve station service transformer $4.16 \mathrm{kV}$ voltages before closing in 12000 Hp motor load during start-up mode, with $50 \mathrm{~A} \mathrm{GIC,} 42.38 \mathrm{mi}$ line and remote source impedance.

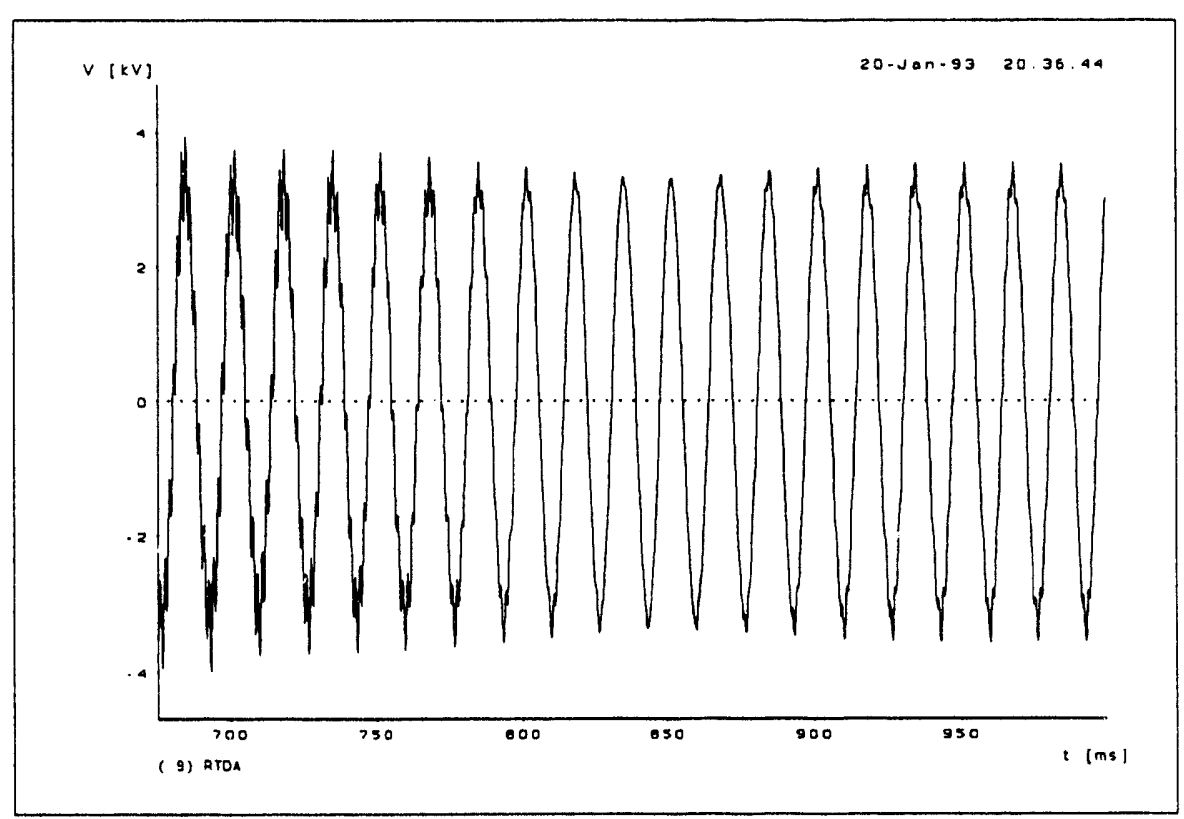

Fig. 7-4. Reserve station service transformer A-phase transient voltage on $4.16 \mathrm{kV}$ side immediately after closing in $12000 \mathrm{Hp}$ motor load during start-up mode, with $50 \mathrm{~A} \mathrm{GIC \text {, }}$ $42.38 \mathrm{mi}$ line and remote source impedance. 


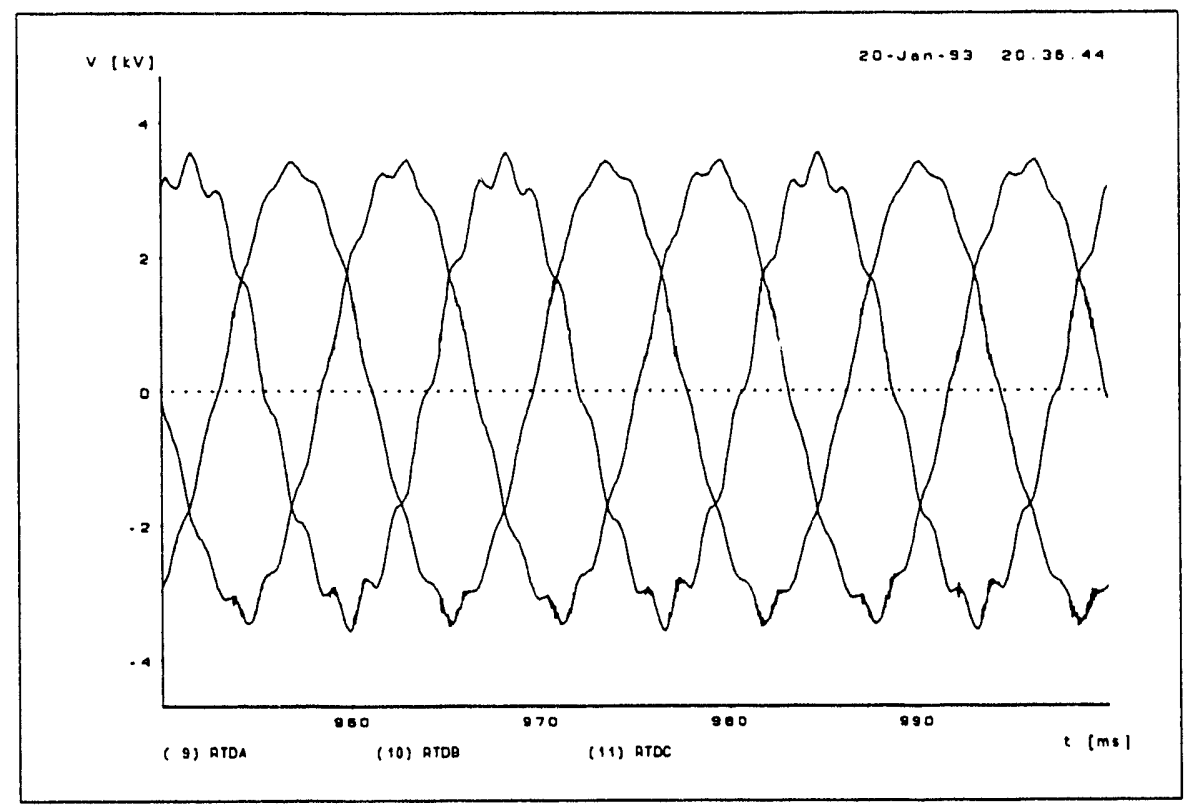

Fig. 7-5. Reserve station service transformer $4.16 \mathrm{kV}$ voltages, $0.3 \mathrm{sec}$ after closing in $12000 \mathrm{Hp}$ motor load during start-up mode, with $50 \mathrm{~A} \mathrm{GIC}, 42.38 \mathrm{mi}$ line and remote source impedance.

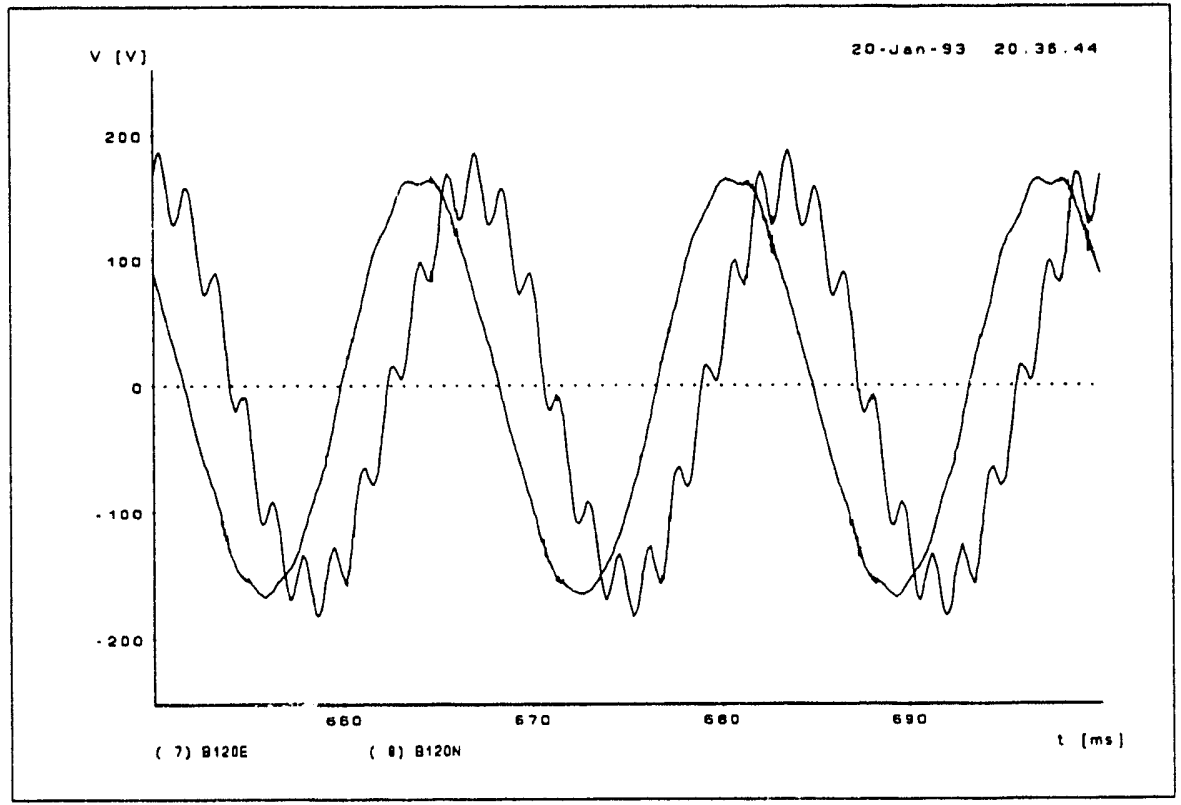

Fig. 7-6. Normal and emergency system voltages at $120 \mathrm{~V}$ buses before closing in 12000 Hp motor load during start-up mode, with $50 \mathrm{~A} \mathrm{GIC,} 42.38 \mathrm{mi}$ line and remote source impedance. 


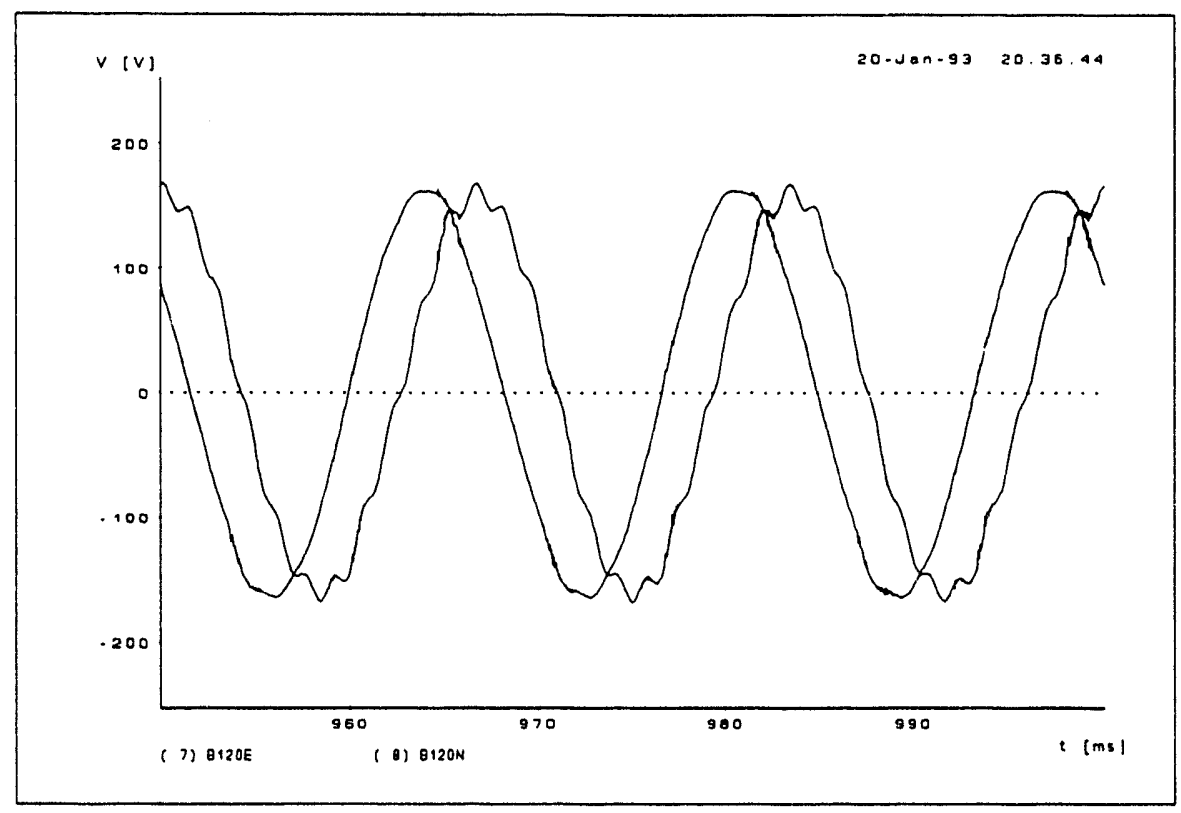

Fig. 7-7. Normal and emergency system voltages at $120 \mathrm{~V}$ buses, $0.3 \mathrm{sec}$ after closing in $12000 \mathrm{Hp}$ motor load, during start-up mode with $50 \mathrm{~A} \mathrm{GIC}, 42.38 \mathrm{mi}$ line and remote source impedance.

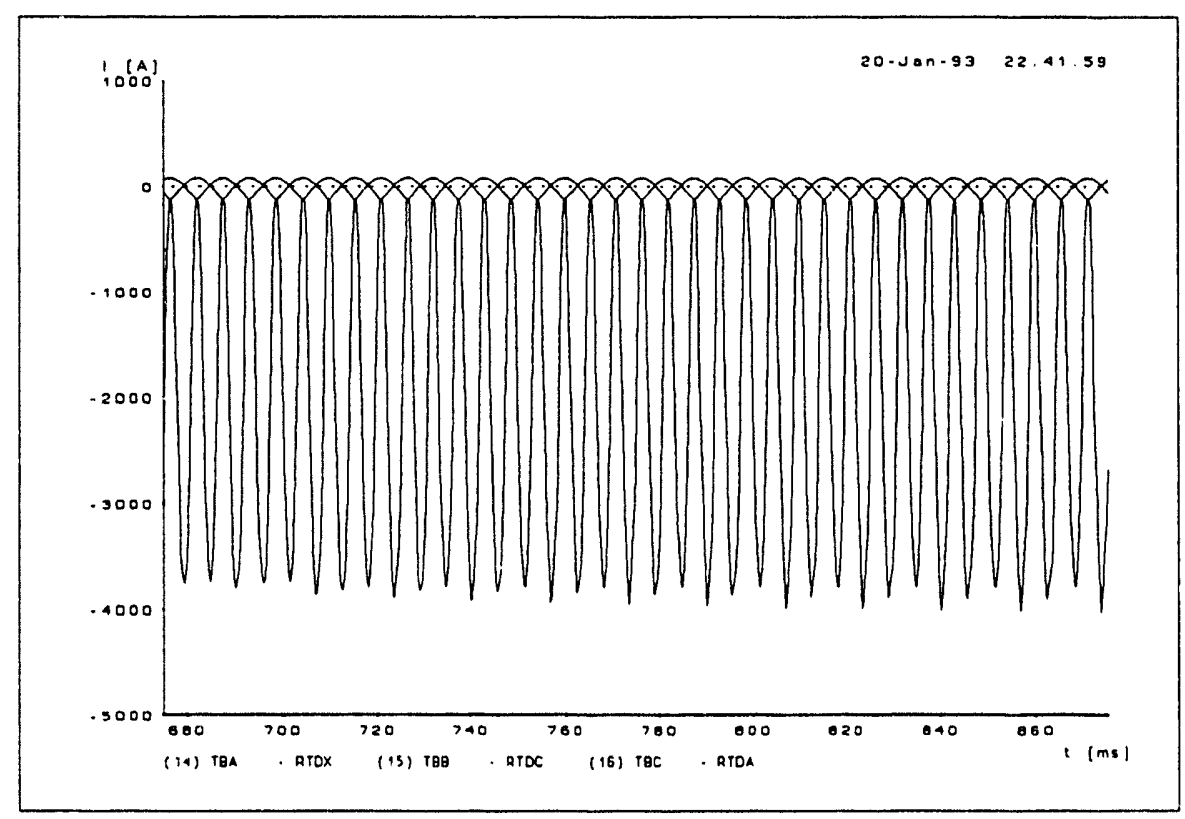

Fig. 7-8. Reserve station service transformer magnetizing currents immediately after tripping $12000 \mathrm{Hp}$ motor load during start-up mode, with $50 \mathrm{~A} \mathrm{GIC}, 42.38 \mathrm{mi}$ line and remote source impedance. 

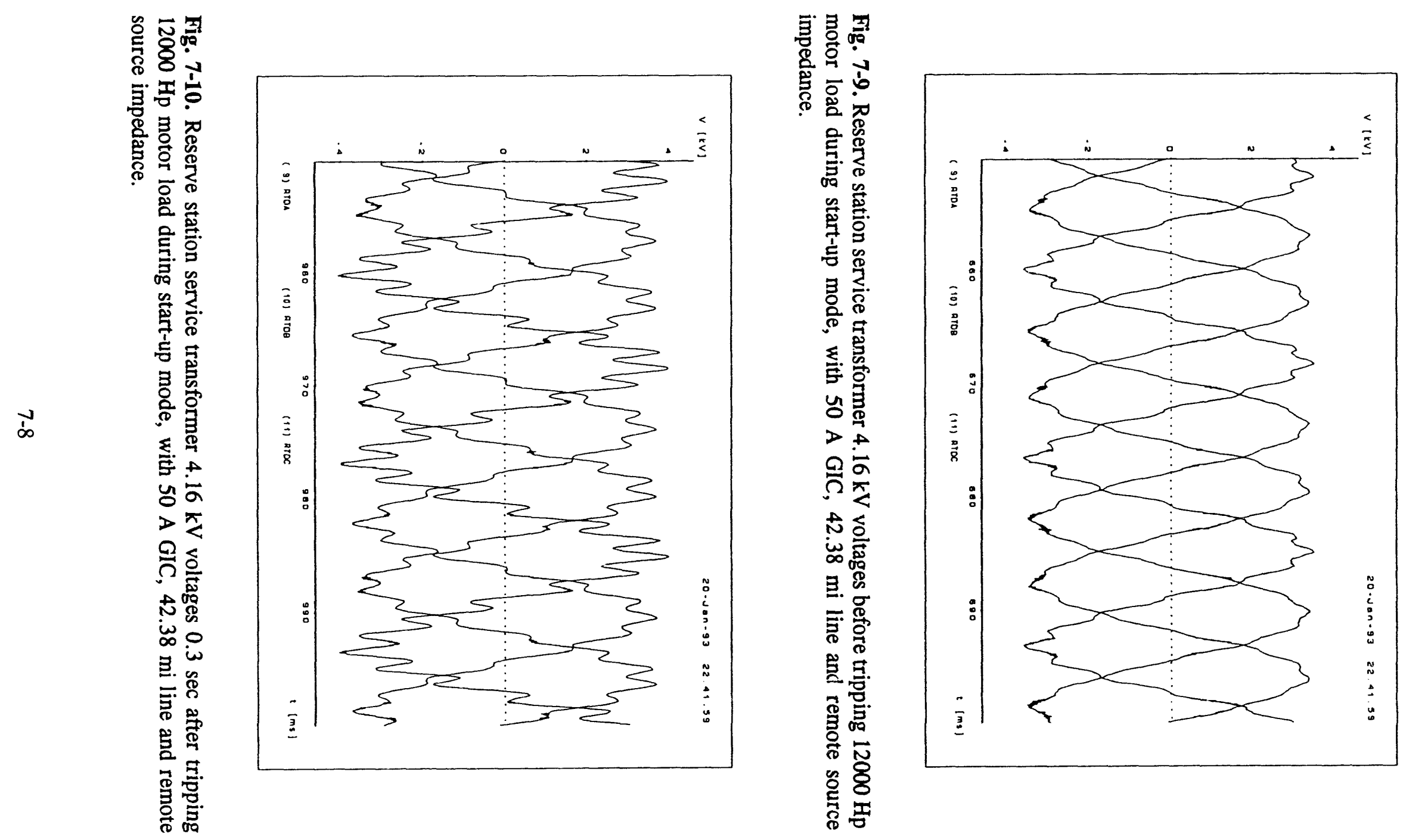


\section{CONCLUSIONS AND RECOMMENDATIONS}

GIC disturbances have the potential to present unique challenges for nuclear power stations. A partial list of the effects of GIC would include thermal degradation of generator step-up transformers, possible overheating of generators due to negative sequence currents in the stator windings, and misoperation of protective relays caused by harmonics from current and power transformers. Additionally, generators strained by the increased requirements for reactive power, exacerbated by the potential loss of static var compensators, will jeopardize the integrity of the power transmission system and increase the likelihood that a nuclear site may have to deal with a loss of off-site power.

\subsection{Conclusions}

Based on this research and the EMTP Simulation performed on a typical plant's electrical distribution system, the following conclusions are drawn:

1. GICs resulting from solar magnetic storms, if unmitigated, may render the in-plant electrical distribution systems in nuclear power plants vulnerable to higher levels of total harmonic distortion at all voltage levels. Mitigation methods are currently being investigated under EPRI Project RP-1770 and tested under RP-3211.

2. The levels of harmonic distortion to be expected depend among others, upon the arrangement of the electrical distribution system, the transmission network parameters, the configuration of the normal station auxiliary and preferred sources, plant operating mode (i.e., normal steady state power operation, start-up or shut down) and bus loading. Minimal attenuation of the harmonics may be expected in going from the high voltage to the low voltage busses in the plant distribution system.

3. High voltage level (above $600 \mathrm{~V}$ ) safety-related busses being lightly loaded, except under emergency operating conditions, may be more vulnerable to higher levels of harmonic distortion than their non-safety related counterpart.

4. Although there is very minimal attenuation of the harmonics between the $4.16 \mathrm{kV}$ and the $120 \mathrm{~V}$ AC voltage levels, the $120 \mathrm{VAC}$ vital instrumentation bus should not experience any effect during periods of high solar magnetic disturbance leading to a GIC presence at the main and reserve transformers.

5. Study of transient conditions such as a loss of, or addition of a major load on $13.8 \mathrm{kV}$ bus (which is also fed by the reserve transformer) with high levels of harmonics due to GIC indicated that in and of itself, the transient event does not contribute to worsening the harmonic levels in the distribution system at the lower voltage levels (below $600 \mathrm{~V}$ ). Indeed the addition of a major load improves the harmonic levels in the distribution system due to the increased transformer load as would be expected. However, even though the loss of a major load does not show any transient effect, it can result in a new steady state condition that results in increased harmonic levels throughout the distribution system. This also is expected because the transformer becomes lightly loaded.

6. Protective relays (both electro-magnetic and solid state relays) without a harmonic filter, which operate purely as a peak detector are vulnerable to false high readings resulting in improper operation even when only a $50 \mathrm{~A}$ GIC is present in the system. However, the transmission 
network and plant electrical distribution system configurations can influence the extent of the net effect both positively and negatively. Relays that operate on average or rms values of the input waveforms are less vulnerable to mis-operation when exposed to bus conditions with a high harmonic levels. Addition of a harmonic filter can eliminate the problem completely. Based on this study, albeit limited in its scope, the one recommendation that can be made is that in undervoltage protective relays used to sense an undervoltage or degraded voltage conditions for starting the on-site diesel generator, consideration should be given to installing an input side harmonic filter, if one does not exist.

\subsection{Recommendations}

1. On the basis of the studies conducted thus far, monitoring the forecasts of geomagnetic storm activities is recommended. Considerations should be given to install instrumentation to monitor the harmonic levels in the various voltage levels of the in-plant distribution system for plants susceptible is GMD. Operators should consider instituting an administrative system to monitor the harmonic levels in the emergency or safety related $4.16 \mathrm{kV}$ and $480 \mathrm{~V}$ busses during periods of high geomagnetic storm activity. The purpose would be both to obtain real world data for future eraluations and to provide timely warning to the plant operating personnel.

2. Studies using plant specific information would be required to gain a better insight into the expected levels of harmonics for various network and in-plant distribution system configurations, and to study the effects of the high harmonic levels on rotating machineries and other distribution system equipment. Such a study can provide a basis for reaching some very specific and definitive conclusions to support the full resolution of the concerns about the impact of GIC in nuclear power plant operation.

3. Through such a study, one can generate the supporting data required to recommend effective schemes for mitigating the effects of GIC in-plant distribution systems. 


\section{REFERENCES}

[1] Albertson, V.D., "Geomagnetic Disturbance Causes and Power System Effects", Effects of SolarGeomagnetic Disturbances on Power Systems, Special Panel Session Report, IEEE, 90TH0291-5 PWR, 1990.

[2] Pirjola, R., "Induction in Power Transmission Lines During Geomagnetic Disturbances", Space Science Review, Vol. 35, pp 185-193, 1983.

[3] Larose, D., "The Hydro-Quebec System Blackout of March 13, 1989", Effects of SolarGeomagnetic Disturbances on Power Systems, Special Panel Session Report, IEEE, 90TH0291-5 PWR, 1990.

[4] EPRI Report EL-4340 on Project 1424-3; "High Voltage Direct-Current Converter Transformer Magnetics," December 1985.

[5] Working Group K11 of the Power System Relaying Committee, "The Effects of Solar Magnetic Disturbances on Protective Relaying", Geomagnetic Storm Cycle 22: Power System Problems on the Horizon, Special Panel Session Report, IEEE, 90TH0357-4 PWR, 1990.

[6] McNutt, W.J., "The Effect of GIC on Power Transformers", Geomagnetic Storm Cycle 22: Power System Problems on the Horizon, Special Panel Session Report, IEEE, 90TH0357-4 PWR, 1990.

[7] Walling, R.A., and Khan, A.H., "Characteristics of Transformer Exciting-Current During Geomagnetic Disturbances", presented at the IEEE/PES 1991 Winter Meeting, NY, NY, February 3-7, 1991, paper no. 91 WM 095-0 PWRD.

[8] Albertson, V.D., Kappenman, J.G., Mohan, N., and Skarbakka, G.A., "Load-Flow Studies in the Presence of Geomagnetically-Induced Currents", IEEE Transactions on Power Apparatus and Systems, Vol. PAS-100, No. 2, 1981.

[9] Kappenman, J.G., Carlson, D.L., Sweeney, G.A., "GIC Effects on Relay and CT Performance", IEEE 9OTH0291-5 PWR, 1990.

[10] Gill, A.S., "Electrical Equipment, Testing and Maintenance", Reston Publishing Company, Reston, Virginia, 1982.

[11] Intentionally left blank.

[12] Electrical Transmission and Distribution Reference Book, Westinghouse Electric Corp., 4th ed., Pittsburgh, PA, 1964.

[13] Transmission Line Reference Book (345 kV and Above), Electric Power Research Institute, 2nd ed., Palo Alto, CA, 1982.

[14] IEEE Working Group on Power System Harmonics, "Power System Harmonics: An Overview," IEEE Trans. PAS, Aug. 1983, pp. 2455-2460. 
[15] IEEE Power System Relaying Committee, "The Impact of Sine-Wave Distortions on Protective Relays," IEEE Trans. LA, March/April 1984, pp. 335-343.

[16] IEEE Power System Relaying Committee, "Fault Induced Wave Distortion of Interest to Relay Engineers," IEEE Trans. PAS, Dec. 1985, pp. 3574-3584.

[17] IEEE Working Group on Geomagnetic Disturbances and Power System Effects, "Geomagnetic Disturbance Effects on Power Systems," IEEE paper 92 SM 511-6 PWRD, presented at the 1992 IEEE/PES Summer Meeting, Seattle, WA, July 1992.

[18] J. F. Fuller, E. F. Fuchs and D. J. Roesler, "Influence of Harmonics on Power Distribution System Protection," IEEE Trans. Power Delivery, April 1988, pp. 549-557.

[19] A. A. Girgis, J. W. Nims, J. Jacomino, J. G. Dalton and A. Bishop, "Effect of Voltage Harmonics on the Operation of Solid-State Relays in Industrial Applications," IEEE Trans. LA, Oct. 1992, pp. 1166-1173.

[20] William F. Horton and Saul Goldberg, "The Effect of Harmonics on the Operating Points of Electromechanical Relays", IEEE Transaction on Power Apparatus and Systems, Vol. PAS 104, No. 5, 1985.

[21] ITE Instruction Manual IB 18.4.7-2, Issue D, "I-T-E Single Phase Voltage Relays", 1981.

[22] GE Instructions GEI-90805D, "Undervoltage Relays", 1981. 


\section{APPENDIX A \\ BACKGROUND ON SOLAR PHENOMENA}

\section{A.1 SOLAR PHENOMENA}

The physics of the sun is a story essentially told by the equations which serve to enumerate the thermonuclear fusion cycle $[A-1]$. The sun has an immense supply of protons, the fuel necessary to support fusion, and thus the ongoing conversion of protons to helium appears roughly as a system in dyn imical equilibrium, or steady state. There are irregularities in the sun's behavior however that create disturbances which manifest themselves as lively storms on the sun's surface. These storms propagate excessive amounts of energy toward the earth. The intimate relationship between the earth's magnetic field and the electric field created by the earth-bound solar plasma is the underlying cause of the earth surface potentials (ESP), which can create problems for utilities' electrical networks.

Sunspots are large magnetic storms that occur on the sun's surface. Plotting the average annual number of sunspots against time reveals a recurring pattern in their frequency [Fig. A-1] with the geomagnetic disturbance cycle lagging by a few years. Approximately once every eleven years the sunspot cycle reaches a relative maximum. Records of sunspot numbers have been kept for over the past two hundred years, and currently, in 1991, we are in the midst of cycle \#22. Although not actually responsible for geomagnetic disturbances experienced on earth, sunspots were known first and the correlation between sunspots and the true causes of disturbances is very high.

The three types of solar disturbances which have an impact upon the earth's geomagnetic fields are solar flares, coronal holes, and disappearing filaments. Solar tlares emit both electromagnetic radiation and subatomic particles (electrons and protons). Coronal holes and disappearing filaments release electrons and protons which, along with the particles from solar flares, constitute the solar wind. Solar flares are of primary concern in terms of the severity of geomngnetic storms caused by solar disturbances. The largest tlares cluster around the peak of the sunspot cycle, although flares close in magnitude with the potential to cause geomagnetic disturbances can occur at any time.

According to Cleveland, et al, [A-2], a solar flare is a tremendous outpouring of energy which appears as a bright spot on the sun. Evidently, this energy release is associated with the conversion of stored magnetic energy from nearby sunspots into particle and electromagnetic radiation. Solar flares exhibit two phases; the first is the impulsive phase which is then followed by a slower thermal phase. The majority of the flare's energy is released during the impulsive phase including: hard x-rays $(30-300 \mathrm{keV})$ which are produced via the bremsstrahlung of electrons; and gamma rays $(0.5-5 \mathrm{MeV})$ associated with the emission of protons. Later in the impulsive phase, as the plasma heats up, soft $x$-rays $(<30 \mathrm{keV})$, extreme ultraviolet (EUV), and optical radiation is emitted. Finally, emissions of microwave and radio frequencies are produced by energetic electrons.

During the thermal phase, the very hot plasma continues to emit soft $x$-rays, EUV, and optical radiation. The plasma then expands outward and a billowing magnetic tield in the solar wind, called a coronal mass ejection (CME). At the peak of thcir activity these tlares occur 50-100 times every day; however, only approximately $20 \%$ of all tlares produce a geomagnetic disturbance [A-3]. 


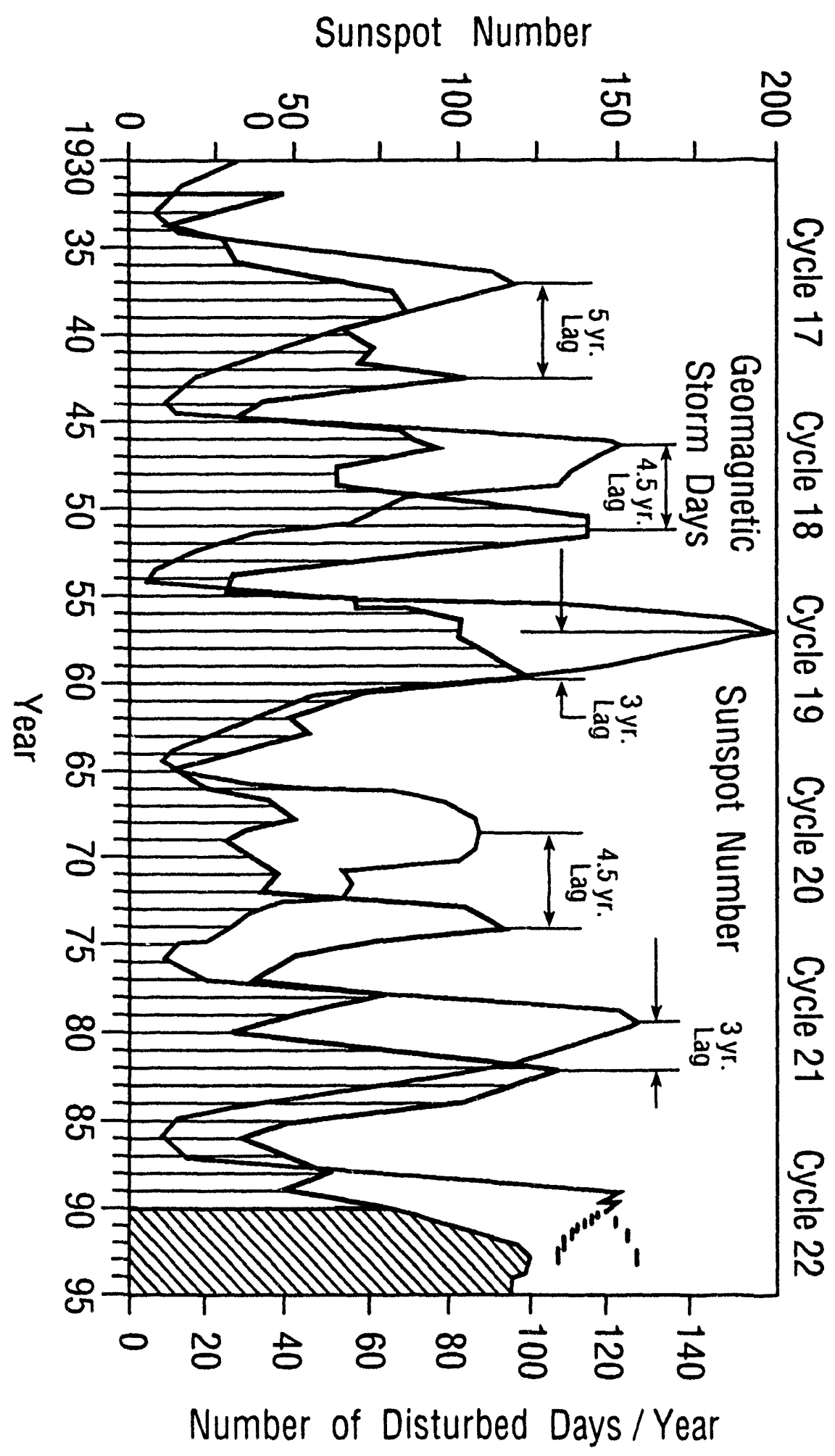


Disappearing filaments are composed of relatively cool, magnetically suspended material which can be observed through special filters and appear as dark stringy features on the sun. Occasionally, changes in magnetic fields result in filament eruptions. The material is spewed out into interplanetary space and can then cause geomagnetic disturbances. Coronal holes are regions where the sun's magnetic fields open into the interplanetary medium and allow particles to propagate away from the sun. Although solar flares and disappearing filaments contribute partially, coronal holes are the primary instigators of the solar wind [A-4], a rarefied plasma of protons and electrons.

The two types of solar ejecta which affect the earth, electromagnetic radiation and subatomic particles (mostly comprising the solar wind), have different transit times. The sun's electromagnetic radiation travels at the speed of light and takes approximately eight minutes to reach the earth. Depending on the energy of the individual particles, they may take anywhere from one to six days to travel to the earth. The solar wind is responsible for the continuously intense dynamic pressure which alters the classical conception of the earth's dipole field. In fact, the earth's magnetic dipole is drawn out into a long tail - roughly $10^{3}$ earth radii - due to the solar wind, and is called the magnetosphere. During periods of intense solar storm activity the releasal of high amounts of particles from the sun may cause an extreme compression and/or deformation of the magnetosphere disturbance monitored by ground stations on the earth [A-5].

Another factor necessary to present a reasonably accurate sketch of the interaction between the solar wind and the terrestrial magnetic field is the interplanetary, or solar, magnetic field. This field is induced by the solar wind itself. A CME will have a magnetic field "embedded" in it which is almost always partially aligned with the earth's magnetic field. With all other factors equal, a CME with a field alignment close to that of the earth's magnetic field will interact more vehemently than a CME with a field unaligned or not closely aligned to the earth's field. This represents an optimal condition for a transfer of energy between the CME and the earth's magnetic tield allowing from geomagnetic storms of greater intensity.

The solar wind coming from the sun will blow around the magnetopause - a boundary between fast wind and trapped magnetospheric plasma - due to the interplanetary magnetic field. Which way a particle circumvents the earth is due to, among other factors, the particle's charge. This is by Lorentz's equation which states that the force on a particle is due to the particle's charge, its velocity, and the direction of the magnetic field; or to be more succinct: $\vec{F}=q \vec{v} \times \vec{B}$. If the interplanetary field is aligned southward, a positively charged particle will flow to the dawn side of the earth while a negatively charged particle will head to the dusk side. Thus, a potential difference of around $100 \mathrm{keV}$ will be created around the equatorial region of the earth on the outside of the magnetosphere.

Through a complex system, field-aligned current can then tlow into the polar caps of the ionosphere. "Auroral Ovals" - rings of enhanced ionization (approx. 3000 - $6000 \mathrm{~km}$ in diameter) - are then produced which are roughly centered on the geomagnetic poles where the aurora commonly appear. These currents, in turn, induce electric fields across the oval which drive an eastward and westward "electrojet" (or auroral current) at about $100 \mathrm{~km}$ altitude. The particles responsible for the auroral current are essentially trapped within the magnetosphere [A-2] but can eventually escape through the polar cusps.

Solar flares, coronal holes, and/or disappearing filaments may increase the speed or density of the solar wind, thereby enhancing the injection of the plasma and thus also, the generation of currents in the auroral zone of the ionosphere. There is no simple relation between the size or position on the sun of a solar disturbance and the intensity of a geomagnetic storm. Most intense storms are associated 
uniquely with long duration (three hours), large $\left(10^{-4}\right.$ gamma) southern components of the interplanetary field near earth $[A-6]$. This is the optimal set of conditions for maximum energy transfer from the solar wind to the earth's magnetic field.

\section{A.2 MAGNETISM}

A rudimentary knowledge of magnetism is necessary to understanc he $:$ : Cs on transformers and other equipment which is used in power transmission or $n$. le a it whis. Most materials may be classified into one of three categories with respect to their magnetic properties: diamagnetic, paramagnetic, or ferromagnetic. Both diamagnetic and paramagnetic materials exhibit magnetism only when in the presence of an applied magnetic field; the instant any such field is removed, the materials relinquish this property. Ferromagnetic materials operate on an entirely different principle. What makes them attractive for use in electrical equipment is their ability to retain their magnetization in the absence of an external field.

The mechanism responsible for ferromagnetism cannot be completely explained without alluding to quantum mechanics; however, a simple qualitative description is all that is necessary here. A ferromagnetic material can be thought of as being composed of minuscule regions, or domains; each domain contains a single atomic sized magnetic dipole. When a ferromagnetic substance is unmagnetized, the domains are oriented randomly, so that they essentially negate each other's effects and no macroscopic magnetic field is produced. In the presence of an applied magnetic field, the tiny dipoles attempt to align themselves in the direction of the tield. When the applied field is discontinued, the domains remain oriented in the same direction and, because they are aligned in the same direction, themselves add to produce a magnetic field.

For a wide range of applied fields $t$ In other words, the stronger the applied fic with the field. This convenient linear relati. beyond a certain point. In an extreme situation where almost every domain is aligned with the field, increasing the applied field, or magnetic force, will not proportionally increase the degree of alignment, or flux density. This phenomenon is known as saturation [Fig. A-2(a)]; as the field strength approaches this limiting value, increasing the field strength by a given amount will have a smaller effect upon alignment of the domains.

Another important concept to take into account is known as hysteresis. When an alternating voltage is applied to the transformer' 1 alternating flux is induced in the core; the magnetic domains are, therefore, constantly realigning themselves. Because of friction, realignment of the domains creates unwanted heating and loss of energy within the transformer's core. Suppose, for example, a sample of ferromagnetic material is partially magnetized, next imagine slowly decreasing the strength in the opposite direction. Although the domains attempt to realign themselves with the new field direction, the resistance present inhibits their ability to achieve total alignment. A sudden reversal of the field direction will not instantly reverse the polarity of the ferromagnetic material because of this resistance. Note also, Figure A-2(b), that as the flux density is increased the area of the hysteresis curve is increased disproportionately, representing an excessive amount of power dissipation due to hysteresis. 

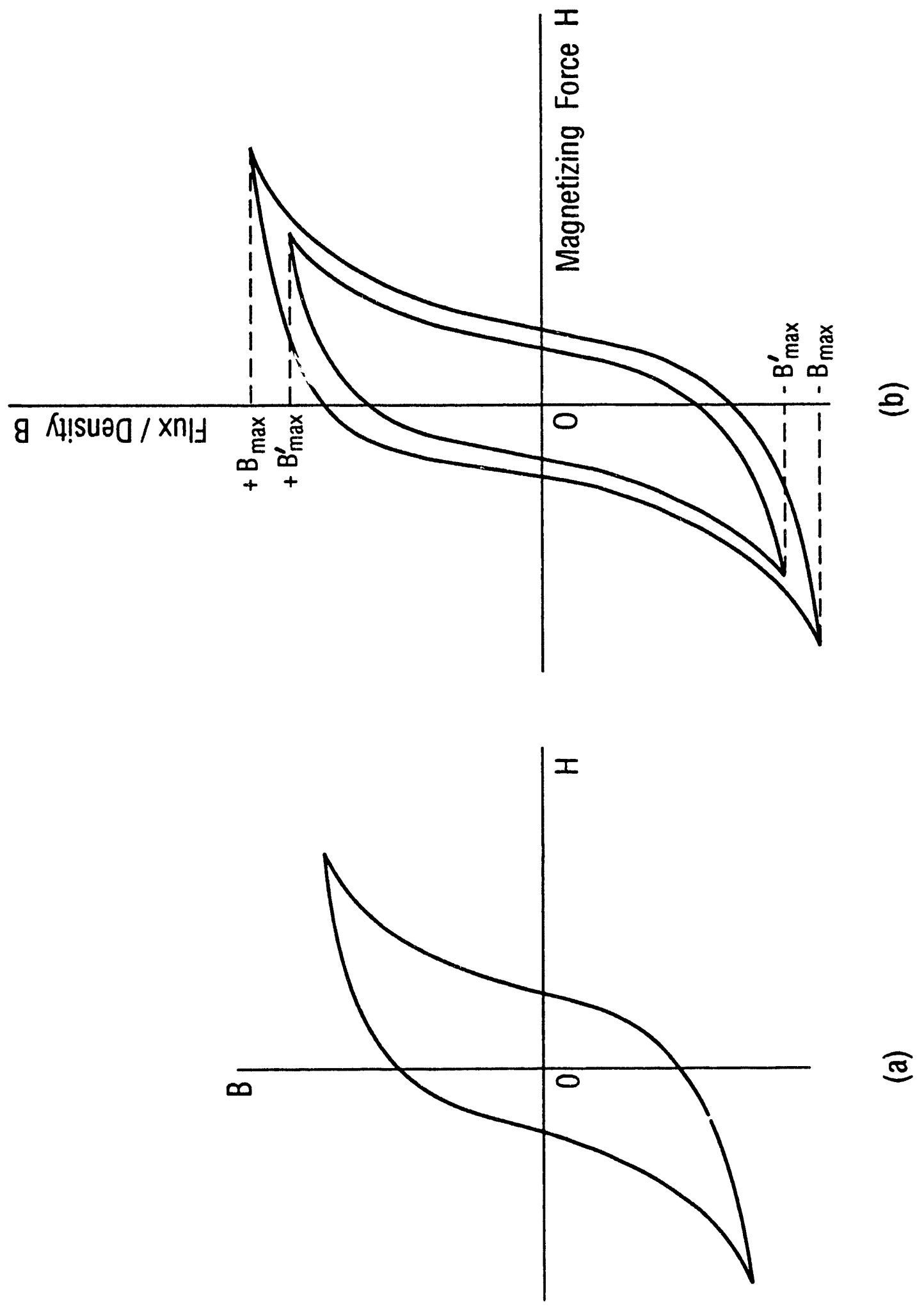

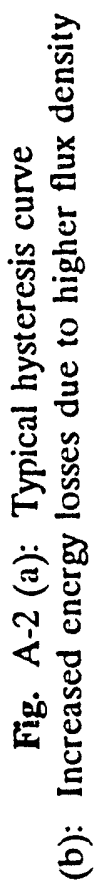




\section{A.3 BASIC PRINCIPLES OF A TRANSFORMER}

A transformer's principle purpose is to change voltage levels for technical and economic reasons. Joule heating losses go as $I^{2} R$, while the power transmitted is $I V \cos ^{2} \phi$, where $\phi$ is the angle between the current and voltage phasers. For a given amount of power, it is best to increase the voltage value rather than the current to minimize losses; however, safety reasons and applications requiring lower voltages prohibit exclusive use of very high voltage lines, thus lower voltages are also used. Transformers can step voltages up, normally from generated levels to transmission levels, and step voltages down, from the high voltage lines to consumer and industry required levels.

The principles behind a transformer's operation need to be examined briefly to understand the troubles that arise from GICs. Given a sinusoidal voltage connected to the primary winding while the secondary winding is open circuited [Fig. A-3(a)] no energy will be transferred (the resistance in this circuit has been neglected because the resistance drop due to the exiting current is negligible compared with the terminal voltage). A small current will flow in the primary winding, appropriately deemed the "no-load" current. As the applied voltage oscillates, this small current flows such that the rate of change of the current, when multiplied by the inductance of the primary winding, generates an electromotive force equal and opposite to the applied voltage [Fig. A-3(b)]. This action immediately prescribes the wave shape of the flux. Since $b$ araday's law a changing tlux induces voltage, and integration could be done on the voltage signal to uetermine the flux's wave form. Finally, the exciting current must conform to a wave shape necessary to generate the needed tlux according to Ampere's law.

The flux's wave shape will be sinusoidal, but, because of the core's ferromagnetic properties, the exciting current will have a highly eccentric wave from [Fig. A-3(b)] that can be determined graphically [Fig. A-4] - hysteresis losses have been neglected in this diagram. The current wave form can be conveniently decomposed into two components: the core-loss component, and the magnetizing component. The core-loss component is in phase with the induced voltage across the primary winding, and is responsible for energy losses due to eddy currents and hysteresis. The magnetizing component is in phase with the flux and is responsible for generating that tlux.

When the transfor mer is under a load, a current will flow in the secondary winding. The resulting secondary current creates its own flux called the secondary leakage flux which, at any time, opposes the main core flux. The consequently lower core flux decreases the induced primary voltage. At this point, there is a greater potential difference between the applied voltage and the primary induced voltage, thereby causing an increase in the primary current. The increased primary current increases the primary magnetomotive force (MMF) which nullifies the effect of the secondary MMF; the transformer reacts very quickly to restore the core flux almost instantly to its no-load level. The core flux drops slightly (1$3 \%$ ) between its no load and full load value however. This decline is necessary if the primary induced voltage is to fall enough to allow a sufficient increase in the primary current.

Just as the current in the secondary winding creates a secondary leakage flux distinct from the core flux, so the current due to the potential difference between the primary winding and the applied voltage sets up a primary leakage flux. Since the core tlux remains virtually constant, the current which sets up that flux must be the same as the no-load current [A-7]. 


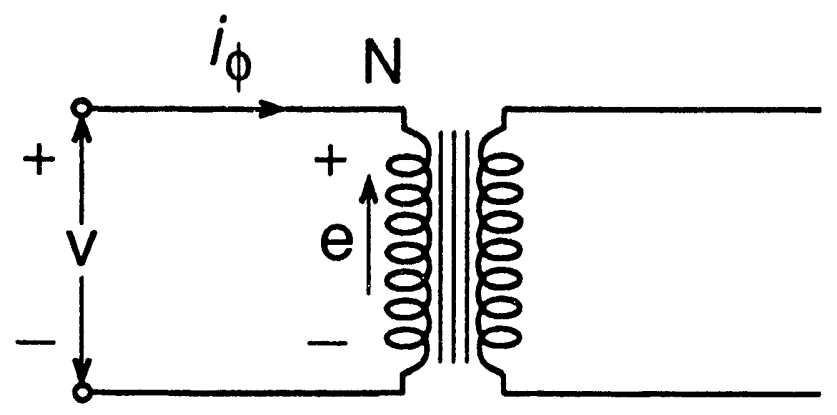

Fig. A-3(a) Transformer with one winding excited from a source of sinusoidal voltage.

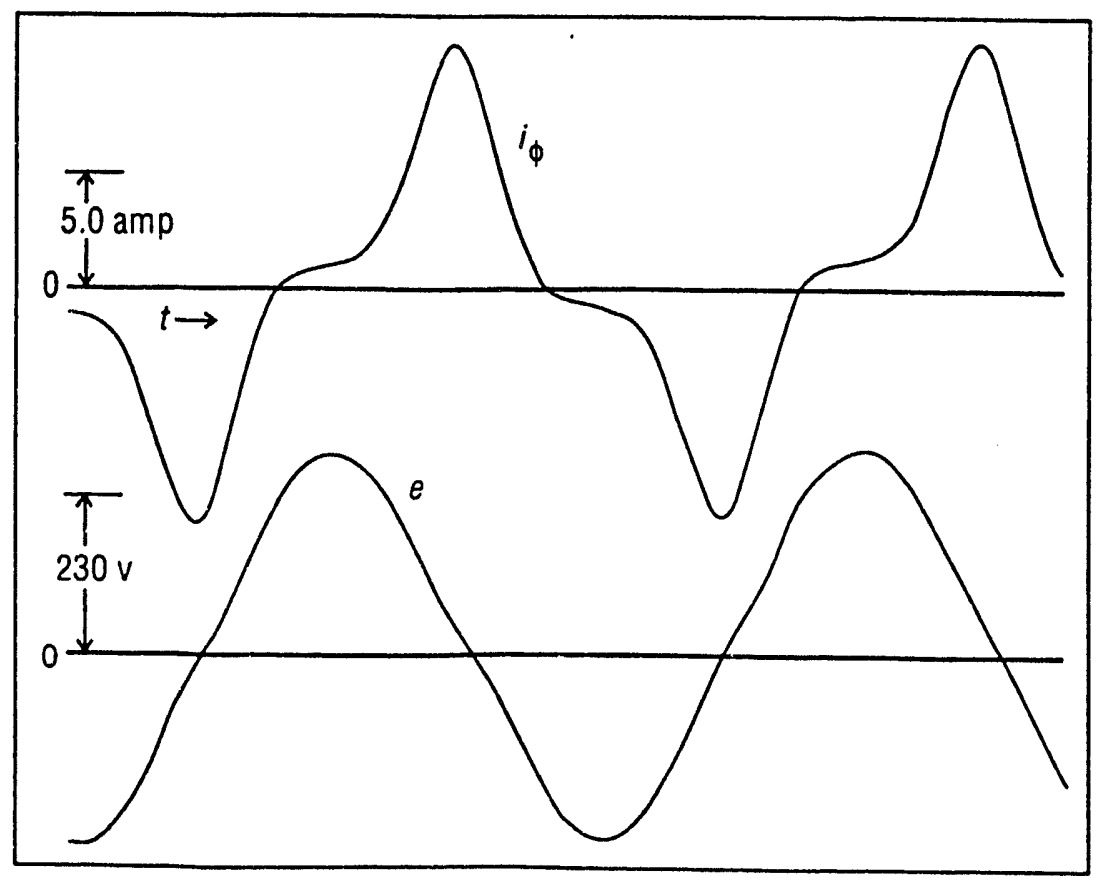

Fig. A-3(b). Exciting-current oscillogram for the transformer with rms induced voltage of 200 volts at a frequency of 60 cycles per second. 
Flux Density

Flux Density $=\mathrm{B}$

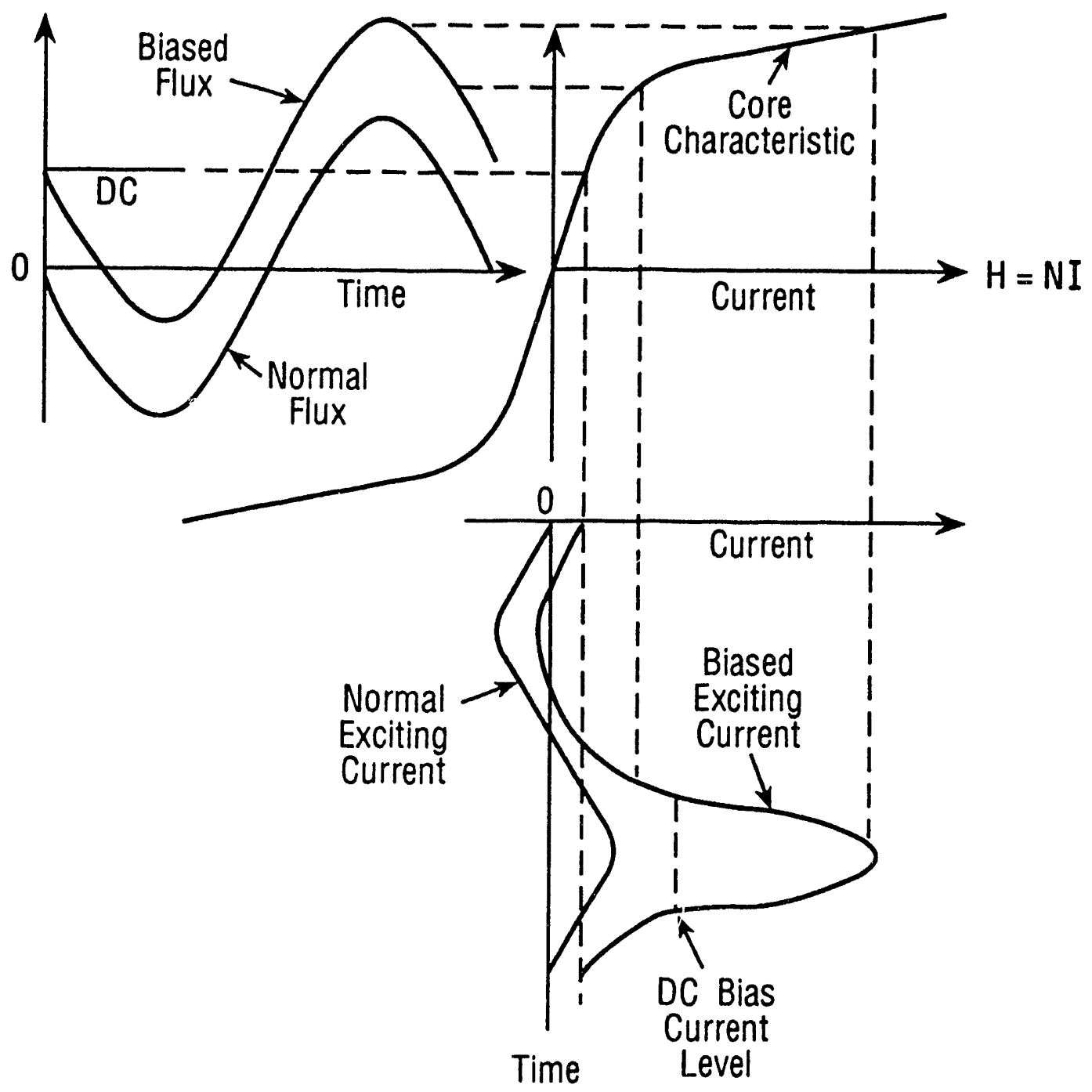

Fig. A-4. Relationship between the magnetic flux and the exciting current for a saturable transformer core for normal operation and bias operation. 
Further analysis of the exciting current can be made using Fourier analysis. According to theory, any periodic function can be represented by a Fourier series - an infinite summation of sine and cosine functions with various frequencies and amplitudes. Though the exciting current may have a peculiar wave form, it is, nonetheless, periodic and therefore lends itself nicely to representation by a Fourier series. The fundamental and third-harmonic components are combined to produce an approximation of the resulting wave from of the exciting current. The wave form also contains higher order harmonics which would make the figure more accurate particularly in the regions near $-15^{\circ}$ and $15^{\circ}$ where points-ofinflection need to be smoothed out. Further investigation reveals that the wave from contains no even harmonics, only odd ones. Also, because the exciting current wave from has neither symmetry about the origin nor about the $y$-axis it is neither an odd nor even function and, thus, has both sine and cosine terms in its Fourier series representation.

The third harmonic is largely responsible for the sharp peak in the wave form of the current. In general, if the maximum flux density is increased, the current becomes more sharply peaked. Increasing the flux density results in a higher percent third harmonic component and a lower percent fundamental component [A-8].

\section{REFERENCES}

[A-1] Krane, K., "Modern Physics", John Wiley and Sons, Inc., New York, New York 1983.

[A-2] Cleveland, F., Malcom, W., Nordell, D.E., and Zirker, J., "Solar Effects on Communications", Geomagnetic Storm Cycle 22: Power System Problems on the Horizon, Special Panel Session Report, IEEE, 90TH0357-4 PWR, 1990.

[A-3] Joselyn, J.A., "Real-Time Prediction of Global Geomagnetic Activity", Solar WindMagnetosphere Coupling, pp. 127-141, Terra Scientific Publishing Company, Tokyo, 186.

[A-4] Balch, C.C, "Real-Time Monitoring and Predicting of Geomagnetic Activity", Effects of SolarGeomagnetic Disturbances on Power Systems, Special Panel Session Report, IEEE, 90TH0291-5 PWR, 1990.

[A-5] Anderson, C.W., Lanzerotti, L.J., and Mac Lennan, C.G., "Outage of the L4 System and the Geomagnetic Disturbances of August 1972". Bell System Technical Journal, Vol. 53, No. 9, November 1974.

[A-6] Gonzalez, W.D. and Tsurutani, B.T., 1987, Planetary and Space Science, Vol. 35, 1101.

[A-7] Ryff, P.F.,Platnick, D., and Karnas, J.A., "Electrical Machines and Transformers: Principles and Applications", Prentice-Hall Inc., 1987.

[A-8] MIT Staff, Dept. of Electrical Engineering, "Magnetic Circuits and Transformers", The Technology Press, New York, 1961. 


\section{APPENDIX B:}

\section{ELECTROMAGNETIC TRANSIENTS PROGRAM (EMTP)}

\section{B.1 OVERVIEW}

Many circumstances require the detailed study of power system transients and abnormal steadystate conditions. These phenomena usually are highly complex and nonlinear by nature, and do not generally lend themselves to theoretical analysis. Instead it is often necessary to use simulation techniques to investigate these conditions. Problem areas of this type include system overvoltages (such as lightning and switching surges), fault currents, non-sinusoidal waveforms (such as harmonics and inrush currents), electromechanical oscillations, and various other types of transients.

The three main tools available for power system simulation are miniature power system models (e.g. TNA or Transient Network Analyzer), analog and hybrid computers, and digital computer programs. The TNA is fast and efficient, but very expensive, and since it is hardwired for specific applications, it is not very adaptable to the study of non-standard equipment or unusual operating conditions. Analog and hybrid computers are more flexible and are also fast, but again are very expensive and also require relatively long set-up times. They also lack portability, so that all studies must be performed essentially at a single location, where the simulation model was originally set up. In contrast, the digital computer simulation is the most flexible, portable, and economical of all the alternatives. This explains the recent trend by the industry toward widespread use of EMTP for studying power system transients. Although inherently slower than the TNA or analog/hybrid approach, the other advantages far outweigh the speed problem, and as newer generation computers become faster the problem may eventually disappear.

EMTP is recognized by the power industry as the only digital simulation program specifically designed to study power system transients. It has a structure and library of component models well-suited for various types of power system investigations. It has been used in many previous studies ranging from high-frequency phenomena such as switching surges to low-frequency phenomena such as subsynchronous resonance. EMTP usage generally falls into two main categories: system design and analysis of operating problems. System design includes areas such as insulation coordination, specification of equipment ratings, protective device specifications, relay design, control system design, and harmonic filter selection. Operating problems include system outages, equipment failures, harmonics and resonance, fault analysis, voltage instability, and many others.

\section{B.2 HISTORY}

EMTP was originally developed by Dr. Hermann Dommel in the late 1960's at the Bonniville Power Administration (BPA). It was initially developed to study switching and lightning surges cn high voltage transmission lines, as reflected by its numerous and sophisticated overhead line models. Since that time it has grown enormously in size and scope of application, and currently the source code contains well over 60,000 lines of FORTRAN, organized into dozens of separate modules. Until 1984, all development was coordinated through BPA, mainly under the direction of Dr. Scott Meyer, but technical contributions were worldwide from the various user groups and individual organizations. In 1984, the EMTP Development Coordinating Group (DCG) was formed, in cooperation with the Electric Power Research Institute (EPRI), in order to establish a more formal path for future program development and maintenance. At the same time another group was formed under the leadership of Dr. Meyer to promote the parallel development of another version of EMTP, called the ATP (Alternate Transients Program). This led to the formation of the Leuven EMTP center and the CAN/AM EMTP User Group 
to support the ATP. Both the ATP and DCG versions of EMTP were based on the same original EMTP program code available at BPA in 1984. The DCG version of EMTP is available through EPRI. The ATP version is available royalty-free in the U.S. and Canada through the CAN/AM User Group.

\section{B.3 PROGRAM DEVELOPMENT}

Three possible levels of program development have been used to expand and enhance the application of EMTP. The first and most obvious method is the development of a library of data input files created for specific applications. These can be in the form of component modules or subsystems, which are assembled manually to form a particular system simulation. This is what most EMTP users do currently. It involves no alterations or additions to the EMTP code. The second approach involves the development of a separate front-end program, again without modifying the EMTP code. The purpose of the front-end program is to create data input files on demand from a preprogrammed library. Although its use is limited to the scope of the library modules, it makes EMTP more user-friendly by looking after data format details and allowing a more interactive approach. The third and most efficient level of development is to modify the source code. This approach is also the most difficult because it requires a good knowledge of the overall EMTP program structure and the theory behind its operation. By modifying the source code it is possible to develop new features and capabilities, while avoiding undesirable assumptions made in earlier development stages.

Both the ATP and the DCG versions of EMTP have undergone significant development since 1984 , although the original BPA program still constitutes the bulk of both current EMTP versions. Both versions have improved documentation, with revised and retyped Rule Books, and periodic newsletters to introduce and explain new development features. Several notebook volumes and bound reports are also available from EPRI for further user support. Both EMTP versions have also experienced a limited amount of modified and added source code. For example, the DCG version contains a new circuit breaker model, an improved line model, and other more recent changes. The ATP has added a new routine called MODELS, which is a more powerful alternative to the original TACS (Transient Analysis of Control Systems) program. Other ATP modifications are currently underway.

Probably the most significant development for both versions of EMTP is the introduction of the PC version. Until recently, EMTP would only run on mainframes and mini-computers. Now the full program is available to run on nearly any DOS-based or OS/2-based personal computers. PC workstations have become so powerful that engineers can now run realistic-sized EMTP problems at their desktop, with all the convenience of other supporting software, such as graphics programs and desktop publishing. The DCG version of EMTP is currently available to run under either OS/2 or Windows. The ATP version will run on any DOS-based PC, but the currently supported version requires 386- or 486based hardware.

\section{B.4 MAIN ADVANTAGES}

Modeling Capability - EMTP is a simulation program specifically designed to model power systems, compared to other general-purpose digital simulation software, which requires a lower level modeling in terms of the basic elements such as the system differential equations. EMTP also allows this level of modeling, but saves much effort by having preprogrammed system-level building blocks available for typical power networks. The following is a partial list of available components: 
- Three-phase synchronous generator, including excitation system and prime mover dynamics

- $\quad A C$ and DC motors, including mechanical system representation

- Linear and nonlinear circuit elements

- Power transformers, including saturation churacteristics

- Voltage and current sources, fixed time-function and dependent sources

- Transmission lines and distribution cables

- $\quad$ Surge arresters and spark gaps

- Circuit breakers, controlled switches, thyristors and power diodes

- Control systems blocks (transfer functions, limiters, analog and digital elements, transducers, A/D and $\mathrm{D} / \mathrm{A}$ converters, etc.)

If a particular system-level model is not available, it can be created by combining lower level elements into equivalent circuits or blocks.

Flexibility - Once the EMTP simulation is set up, it is quite flexible for changing either the structure or the system parameters. Altering the configuration simply requires adding or removing data lines or blocks in the data input file. "INCLUDE" files are also allowed for convenience in modularizing the simulation. System and component parameters are changed by simply changing the numerical data on the appropriate line of the input file. Numerical scaling of data is never required, a'though per unit system data can be used if preferred.

Portability - EMTP is used worldwide and is considered the utility industry standard for transient simulatioil. It has versions available for many different computers and operating systems, from mainframes to PC's. If a given computer version is not currently available, the FORTRAN source code can usually be compiled without difficulty to create a new adaptation. All versions use the same input data format, so models developed on one computer are always compatible for use on a different computer.

Auxiliary Programs - In addition to the variety of simulation modules available in the main program, EMTP also provides auxiliary programs to compute input data for some of the models, where data is difficult to find. Below is a list of the more important auxiliary programs available:

- $\quad$ Line constants program

- Cable constants program

- Transformer impedance matrix program 
- Magnetic saturation program

- Arrester data program

There are also some auxiliary routines built into the main program, such as a load-flow routine, and a data conversion program in the synchronous generator model, which translates standard machine data into a $D-Q$ axis equivalent circuit representation.

Application Variety - EMTP has been used for a wide range of applications from high-frequency switching and lightning surges to low-frequency electromechanical oscillations. This is due to the wide variety of model components available. Below is a partial list of possible applications:

- $\quad$ Switching and lightning transients

- $\quad$ Capacitor switching

- Balanced and unbalanced faults

- Harmonics and filtering studies

- Transformer ferroresonance

- Control system design and testing

- Power conditioning circuits

- $\quad$ Adjustable speed motor drives

- Static VAR compensation

- $\quad$ HVDC transmission systems

- Electromechanical transients and resonance

Output Capability - Several forms of useful output aata is available directly from the main program, as listed below.

- Tabulated transient data versus time

- Waveform (time plot) files of system variables

- Tabulated voltage and current peaks

- S* dy-state oprorating data

- $\quad$ Fourier series components

- System frequency scan data 
In addition, auxiliary graphics programs PCPLOT and TPPLOT accept standard EMTP output plot files and support a variety of screen graphics modes, as well as many popular printer formats. These programs were written to directly interface with EMTP. They also produce HPGL and Postscript graphics files, which directly import into WordPerfect and many desktop publishing packages, for ease in creating engineering reports on a PC or workstation.

\section{B.5 PROGRAM DESCRIPTION}

\section{Program Structure}

- $\quad$ Power network (main program)

- TACS (control systems, special functions)

- Network/controls interface

- Auxiliary (supporting) routines

\section{Available Subsystem Blocks}

- Power Network:

generators/motors, transmission lines and cables, lumped R-L-C elements, circuit breakers and switches, transiormers, thyristors, diodes, $\mathrm{AC}$ and $\mathrm{DC}$ sources, etc.

- TACS: transfer function blocks, logic functions, limiters, analog and digital elements, measurement transducers, A/D and D/A converters, custom FORTRAN CODE, special functions and signals, etc.

- Interface: generator excitation control, circuit breaker control, relay algorithms, thryristor gate pulses, voltage and current feedback measurements, etc.

Input Data File - The model system is determined by the in rut data file. This file specifies the simulation output data, the branch/node connections, the types of models used, and the system component model data. It also determines the sequence of events to occur in the simulation.

\section{B.6 EMTP INPUT DATA REQUIREMENTS}

EMTP input data requirements are very stringent. Since the component models are quite detailed, the user must often obtain data directly from the equipment manufacturer. The required data is sometimes proprietary and is often in a non-standard format. This is particularly true for electric machines, complex feedback controls, transformer nonlinearities, overhead transmission line and distribution cable parameters. Input data acquisition for EMTP can therefore become a time-consuming process, and often constitutes a major part of the overall set-up time. The auxiliary programs help in this regard by translating available data into the required EMTP format.

\section{Synchronous Generator}

Electrical:

1. Steady-state operating voltage and frequency.

2. Winding configuration: delta, grounded or ungrounded wye connected.

3. Saturation characteristic: at least 2 points on the no-load terminal voltage vs. field current curve. 
4. Number of poles, nominal MVA and voltage rating.

5. Stator resistance and leakage reactance.

6. $\quad D$ and $Q$ axis synchronous reactance.

7. $\mathrm{D}$ and $\mathrm{Q}$ axis subtransient and transient reactance.

8. D and $Q$ axis open-circuit subtransient and transient time constants.

9. Zero-sequence reactance.

10. Neutral impedance (if any).

Mechanical:

1. Rotational inertia of rotor/prime mover shaft system.

2. Torque input characteristics of prime mover and governor.

Exciter:

1. Block diagram of excitation control system.

2. Gains, time constants and saturation characteristics.

\section{Overhead Transmission Lines}

Preferable data:

1. Positive and zero sequence impedance and capacitance matrices per meter.

2. Length of each line segment to be represented.

3. Frequency dependence of line parameters.

Alternate data:

1. Conductor construction and cross-sectional dimensions

2. Geometric conductor/tower layout and dimensions.

3. DC resistance of each conductor and shield wire.

4. Earth resistivity over transmission route.

5. Frequency range of interest in the line simulation.

6. Length of each line segment to be represented.

\section{Distribution Cables}

Preferable data:

1. Positive and zero sequence impedance and capacitance matrices per meter.

2. Length of each cable segment to be represented.

3. Frequency dependence of cable parameters.

Alternate data:

1. Cable construction: single-core or pipe-type.

2. Conductor/sheath grounding information.

3. Geometric conductor layout and dimensions.

4. Relative permeability of pipe. 
5. Relative permittivity of internal dielectrics.

6. Resistivities of sheaths, core and surrounding medium.

7. Length of each cable segment to be represented.

\section{Distribution Loads}

Non-rotational (lighting, heating, electronic, small motors):

1. Constant impedance equivalents at each bus.

Major induction motor loads:

1. Rotor and stator resistance.

2. Rotor and stator leakage reactance.

3. Mutual (magnetizing) reactance $\left(\mathrm{X}_{\mathrm{m}}\right)$.

4. Number of poles, rated power and voltage.

5. Rotor and load inertia.

6. Torque/speed characteristics of load.

\section{Converter Circuits}

Power circuit:

1. Converter topology.

2. Switching device type(s) or Mfr data sheet(s).

3. Values of auxiliary elements (snubbers, commutation circuits, etc.)

Control system:

1. Block diagram layout with explanation of various control modes and functions.

2. Control parameters (time constants, gains, nonlinearities, etc).

3. Sample gate pulse waveforms transmitted to power circuit.

4. Feedback signals from power circuit.

\section{Circuit Breakers and Switches}

\section{Mechanical:}

1. Interruption sequence and timing.

2. Energizing and reclosing sequence, pole span.

3. Auxiliary circuit parameters (if any).

Solid-state:

1. Circuit topology and switching device type.

2. Auxiliary circuit parameters.

3. Control sequences and timing. 


\section{Transformers}

1. Winding configurations (delta, grounded/ungrounded wye).

2. Primary, secondary and tertiary resistance.

3. Primary, secondary and tertiary leakage reactance.

4. Magnetizing reactance and core losses.

5. Saturation (flux vs. current) characteristic.

6. Rated voltage and power.

\section{Surge Arresters}

1. Nominal voltage rating and power handling capacity.

2. Point-by-point I-V characteristics over operating range.

\section{B.7 EMTP EXAMPLE SIMULATIONS}

In order to illustrate the capability and versatility of EMTP, three sample simulations are described in Appendix C. The first simulation involves the study of switching transients associated with the energizing of a high voltage transmission line. This example reveals the statistical nature of this problem, and how it can be controlled with preinsertion resistors and appropriate timing of the respective pole closing points. The second example is a transformer simulation study, which includes the effects of core saturation and hysteresis on the excitation current waveforms. The third example is a study of the transient interaction of a nuclear plant generator with a power network during a severe system disturbance. This simulation is modeled according to an real event that occurred recentiy, which resulted in severe damage to the generator. The study described here was an attempt to explore what actually happened to the machine during various scenarios of the disturbance event. A very detailed representation of the generator, the exciter and the mechanical system was required, along with a good equivalent model of the transmission network and the remote sources.

In each of the example simulations in Appendix C, there is a brief system description of the system, followed by a listing of the EMTP data input file, and finally a series of plotted waveforms that illustrate system behavior during the transient event. Details of the EMTP data input files may be difficult to follow for those unfamiliar with the program, however, numerous comment lines have been added to get a general idea of the input data format. 


\section{APPENDIX C}

\section{SAMPLE EMTP STUDIES}

\section{C.1 THREE-PHASE TRANSMISSION LINE ENERGIZATION}

In a power network it is often necessary to energize a transmission line which is temporarily disconnected at the receiving end. Depending on when the breakers close and other factors, this could lead to transient overvoltages on the line conductors and on equipment connected at the generating facility (sending end). It is problem that needs careful attention so that station insulation designs are adequate and safe operating procedures can be established. Since this problem is statistical by nature and depends on a number of factors such as pole closing times (relative to the $60 \mathrm{~Hz}$ wave). The pole span is defined as the time span between the first and last breaker to close in the three-phase sequence. The pole span is also statistical relative to the aiming point, because most breakers do not make contact precisely at the desired time. If preinsertion resistors are used to help damp closing transients, then resistor size, the insertion times and insertion intervals also affect the magnitude of closing transients. For a three-phase line the numerous closing parameters and the statistical nature of the problem makes a computer simulation essential for meaningful design study. The following example illustrates this application of EMTP for a single three-phase line energization. The four cases shown illustrate the wide range of overvoltage results that can be obtained. A more realistic study may involve several lines and would require a larger number of runs (perhaps 200 or more) to generate a statistical data base for variations in the aiming point, pole span, and preinsertion resistor timing. EMTP has special programmed switching sequences designed for this purpose.

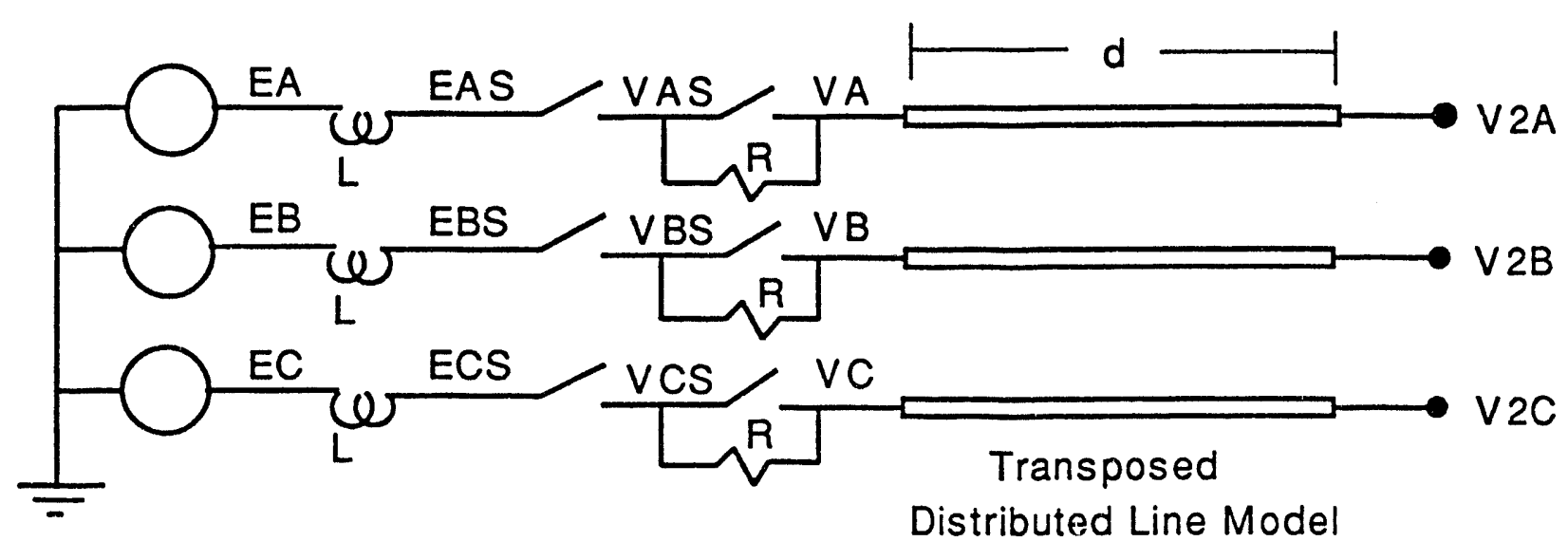

Fig. C-1. Three-phase line closing circuit. 


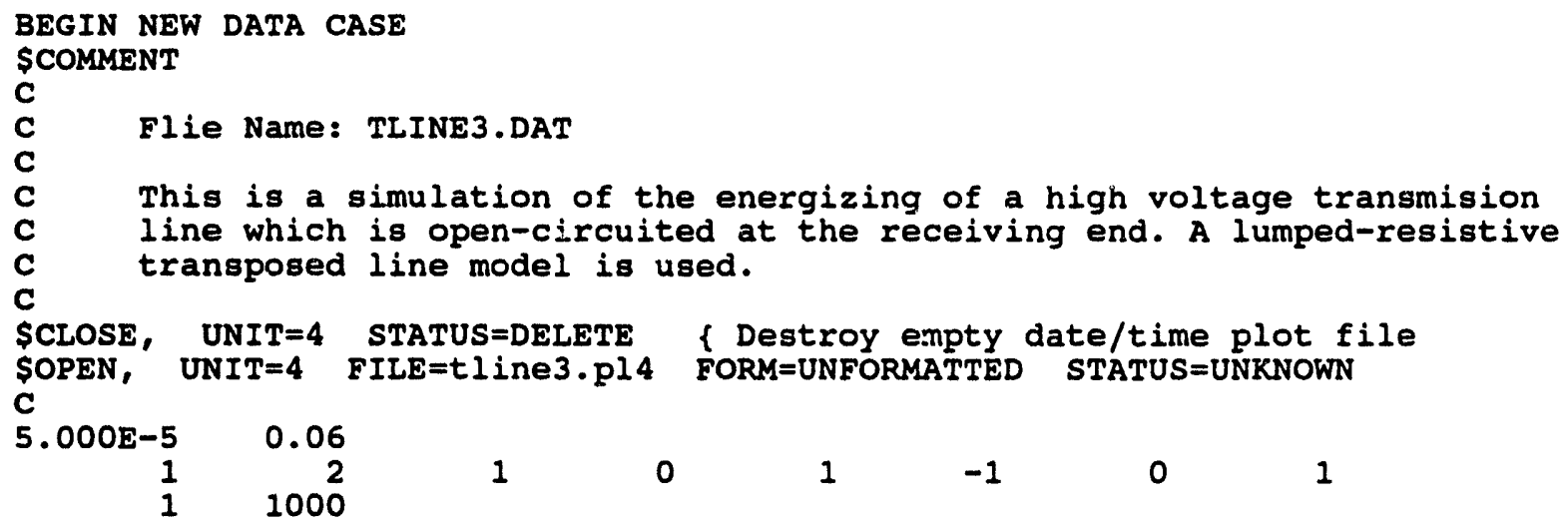

BLANK END OF SWITCHES

C

C AC source voltage.

C

$\begin{array}{lllll}14 \mathrm{EA} & 1408248 . & 60.0 & -90 . & 0.0 \\ 14 \mathrm{~EB} & 1408248 . & 60.0 & -210 . & 0.0 \\ 14 \mathrm{EC} & 1408248 . & 60.0 & 30 . & 0.0\end{array}$

C

BLANK END OF SOURCES

C

C Nodes for plotting.

$\begin{array}{lllllllll}\text { EA } & V A & V 2 A & E B & V B & V 2 B & E C & V C & \text { V2C }\end{array}$ C

BLANK END OF PLOT NODES

BLANK END OF PLOTS

BLANK END OF SIMULATION 
CASE 1

Variable !naxima: Times of maxima : Variable minima : Times of minike :

EA

VA

V2A

EB

VB

V2B

EC

ve

v2C

408248. 563481.707707240 .939408247 .105439265 .767497075 .266408247 .105440257 .929509439 .185

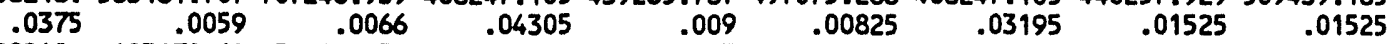
$\begin{array}{lllllllll}-408248 & -483170.68 & -588402.54 & -408247.1 & -436305.73 & -491613.68 & -408247.1 & -495342.87 & -690441.92\end{array}$

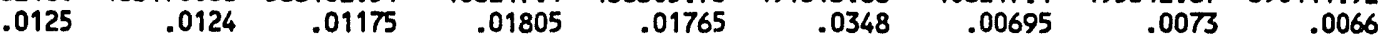

\section{CASE 2}

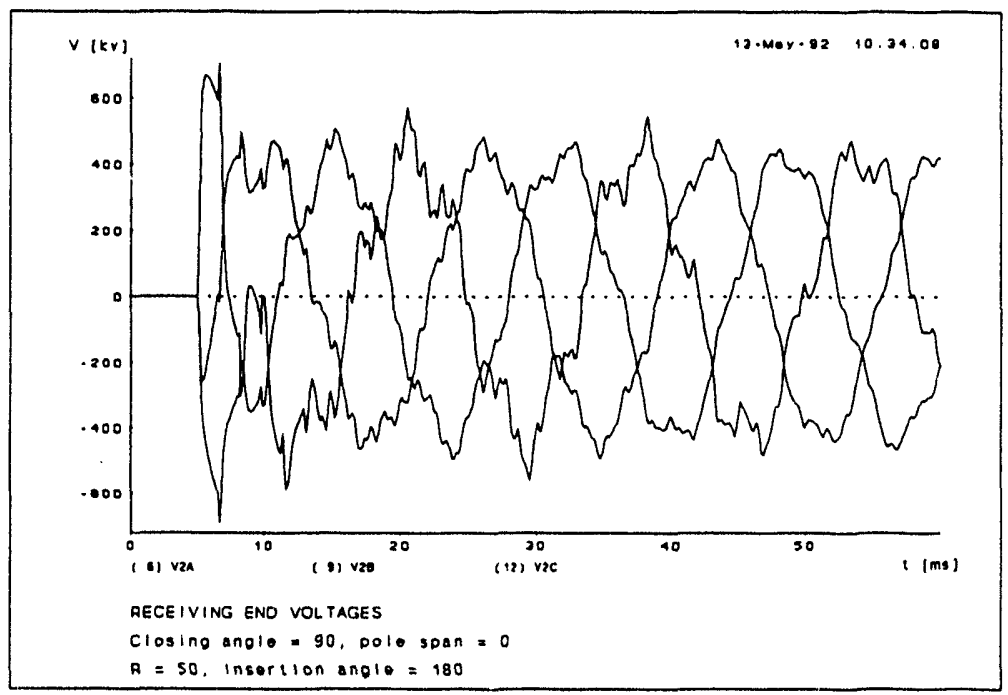

Variable maxima : Times of maxima : Variable minima : Times of minima :

EA

VA

V2A

EB

VB

V2B

EC

vc

V2C

408248. 440345.741526962 .689408247 .105434918 .992512102 .542408247 .105448155 .041532004 .602 0375 $-40375$ .0066 .0125

$-420778.12-453003.94$
$\begin{array}{rr}.04305 & .0269 \\ -408247 & .02705 \\ .046120 .15 & -532177.02\end{array}$ $\begin{array}{rrr}.01805 & -446120.15 & -532177.02 \\ & .0178 & .01835\end{array}$ .03195
-408247.1 .00695 .03205 $-466198.2-542258.82$ $.00735 \quad .02355$

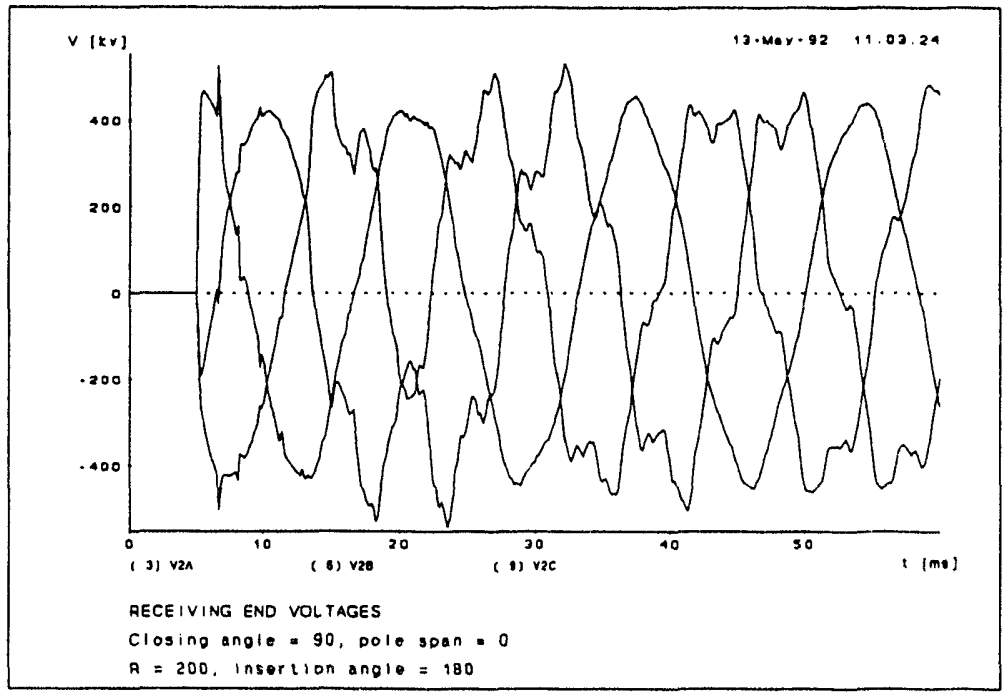


CASE 3

$\begin{array}{lllllllll}E A & V A & \text { V2A } & E B & \text { VB } & \text { V2B } & \text { EC } & \text { VC } & \text { V2C }\end{array}$

Variable maxima : Times of maxima : Variable minima :

408248 .0375 $-408248$.

.0125

rimes of minima :
433061.595590794 .421408247 .105449394 .123604817 .926408247 .105432775 .416523707 .228 $\begin{array}{llllllll}.03715 & .0066 & .04305 & 0.0108 & 0.01075 & .03195 & .0157 & .0158\end{array}$

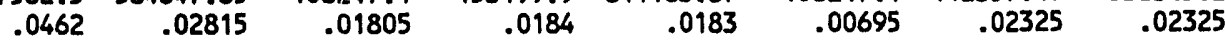

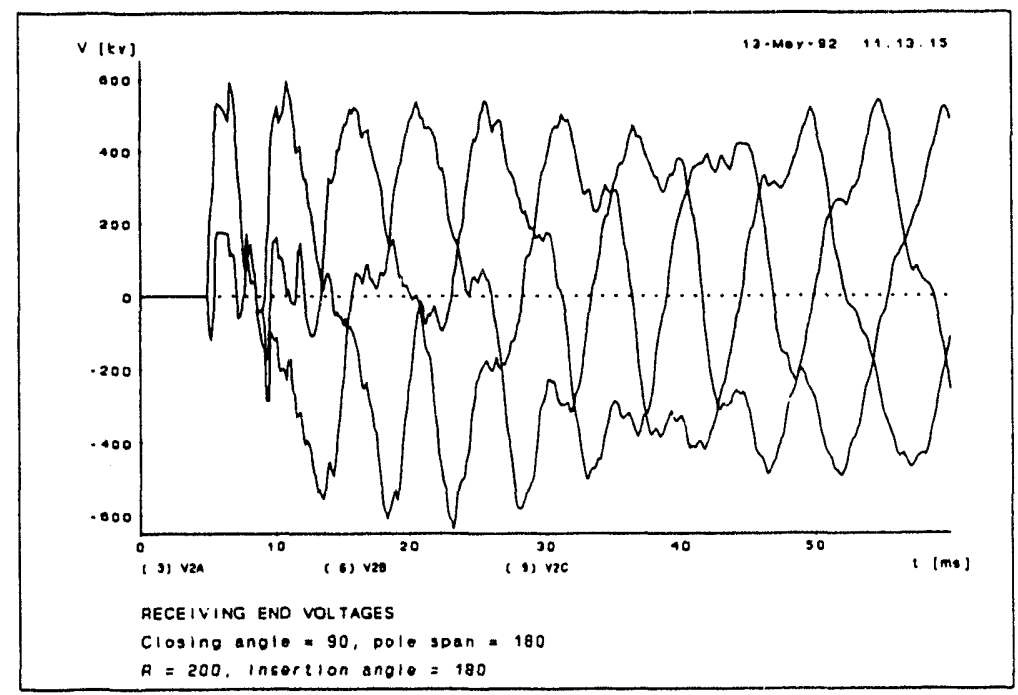

CASE 4
EA
VA
V2A EB
VB V2B
EC
ve
v2c

Variable maxima : Times of maxima : Variable minima : Times of minima :

408248. 418600.737 .0375 $-408248$ .0207 476630.36408247 .105423357 .074490273 .799408247 .105422143 .103470192 .425

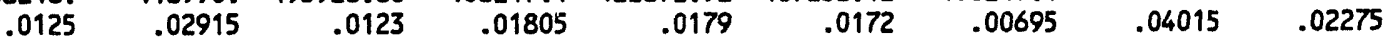

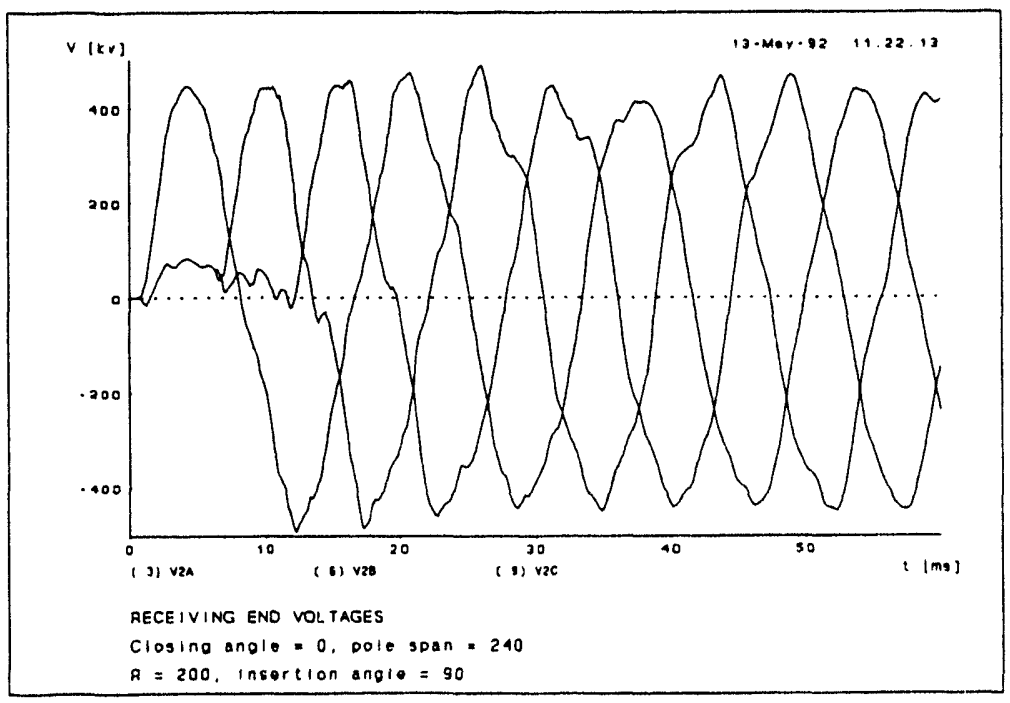




\section{C.2 EFFECTS OF TRANSFORMER SATURATION}

It is often necessary to represent the effect of transformer saturation in the simulation of power system transients. This nonlinear property of all iron-core transformers can produce steady-state harmonics and large transient currents in a power system. These effects would not show up using a linear (ideal) transformer model, thus saturation must be carefully modeled from the manufacturers test data in order to accurately predict these phenomena. EMTP has several models available for transformer simulation, along with supporting routines to convert standard manufacturers test data into the correct data format for EMTP. The following examples illustrate the use of one type of transformer model (TRANSFORMER) for a simple transient study. The diagram below shows the equivalent circuit for this EMTP model of a single-phase, two-winding transformer. A three-winding model would include a third branch connected across the magnetizing branch. A three-phase model is comprised of three separate single-phase units connected at the terminals in either a wye or delta configuration. Also shown below is the saturation characteristic of the simulated transformer. The EMTP routine SATURA converts manufacturers RMS excitation data into flux-linkage vs. current data required by the EMTP TRANSFORMER model.

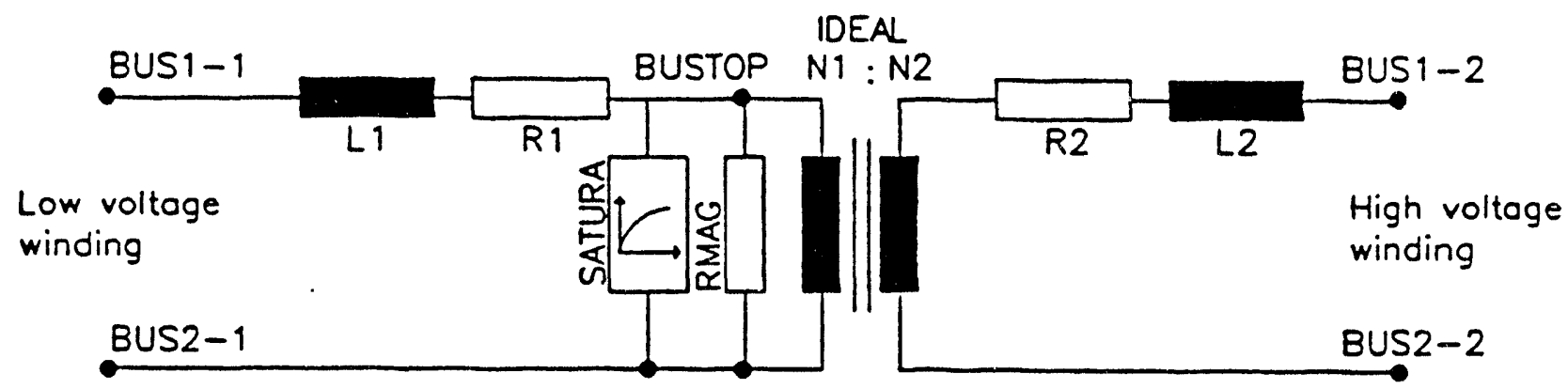

Fig. C-2. Equivalent circuit for single-phase, two-winding EMTP TRANSFORMER model.

RMS Excitation Dato

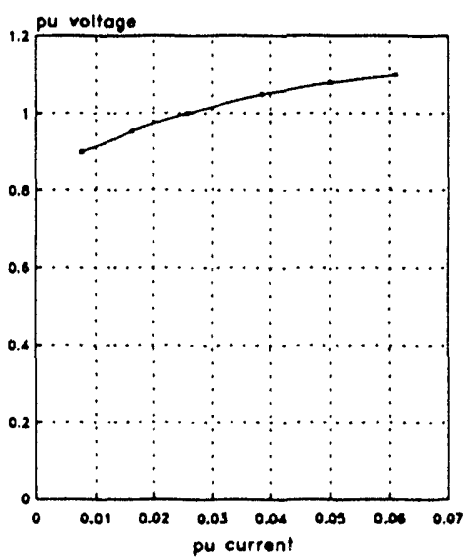

Saturation Curve

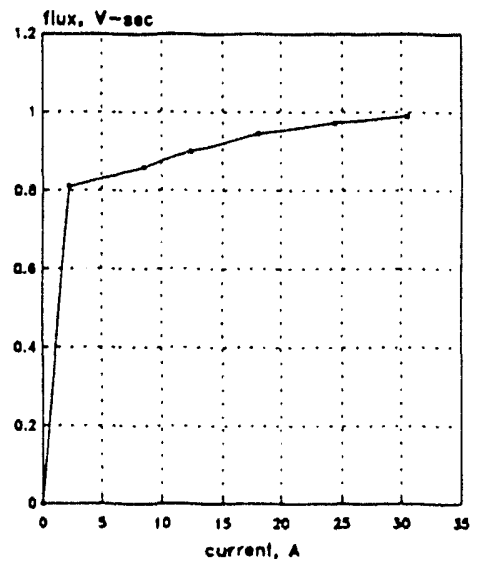

Fig. C-3. Saturation characteristics for example transformer simulation. 
CASE 1: Single-phase transformer with saturation. Transformer is initially excited and the load is switched in after 5 cycles.
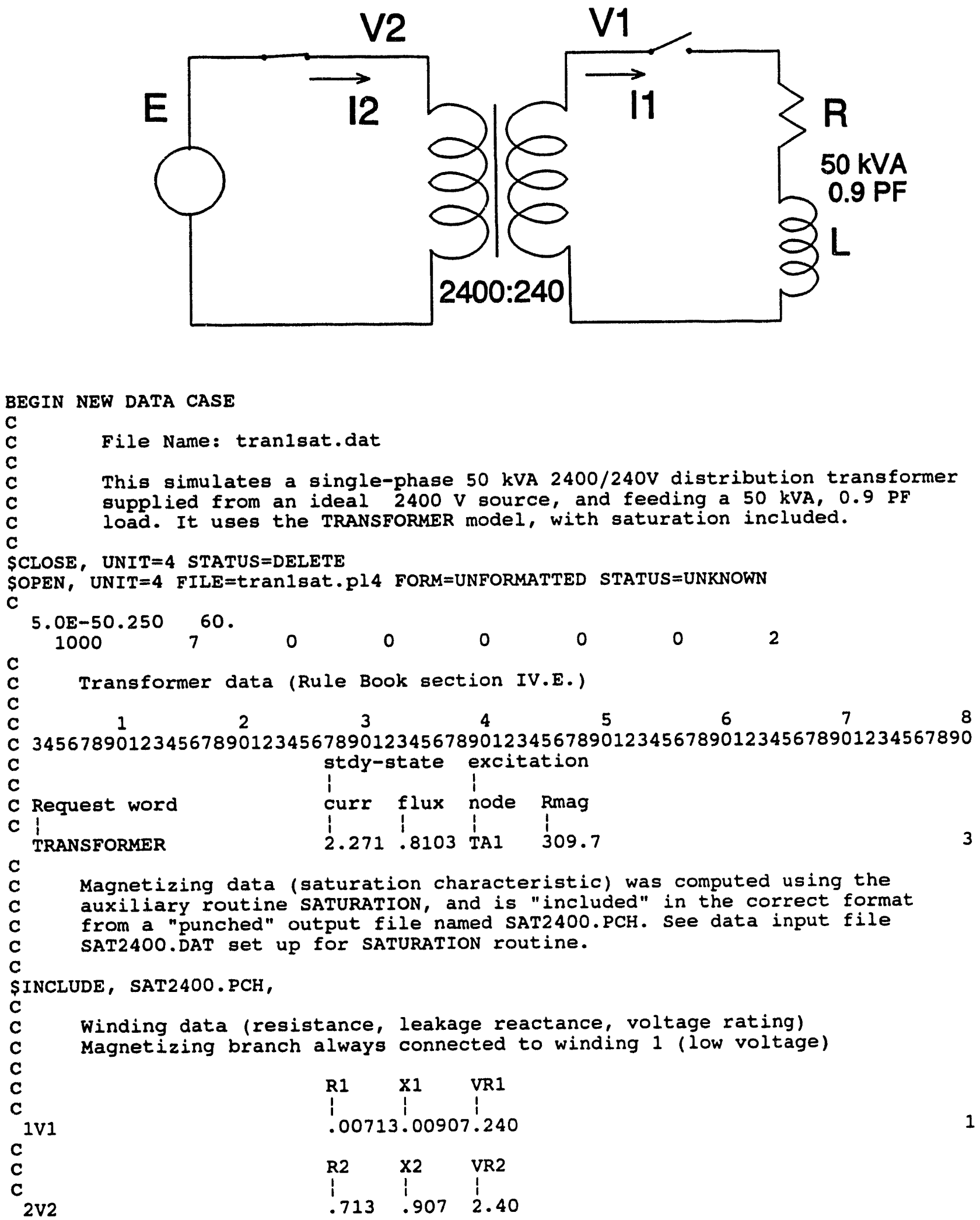
C Load Data

C

BLANK END OF BRANCH DEFINITIONS

C

C Energizing switch

C

V1 VL .083333310 .

c Measuring switch

E V2

MEASURING

C

BLANK END SWITCH CARDS

C

c

$14 \mathrm{E}$

source data

3394.0

60.0

0.0

0.0

$-.0001$

BIANK END OF SOURCE DEFINITIONS

C

C Node voltages for plotting

C

E V2 V1

C

BLANK CARD ENDING PLOT CARDS

C FOURIER ON

C 1941.7 .4324 .1

C 1941.7 .4324 .1

C 1841.7 .4324 .1

I 30

BLANK END OF SIMULATION

BEGIN NEW DATA CASE

BLANK END OF ALL CASES

CASE 2: Transformer is excited at $12.5 \mathrm{msec}$ (to simulate transient inrush current), then the load is switched in after 5 cycles. Modified portion of EMTP data input file id shown below:

C Energizing switch

V1 VL $\quad .083333310$.

C

Magnetizing switch

E V2 $\quad$ V $0125 \quad 10$.

1

BLANK END SWITCH CARDS 

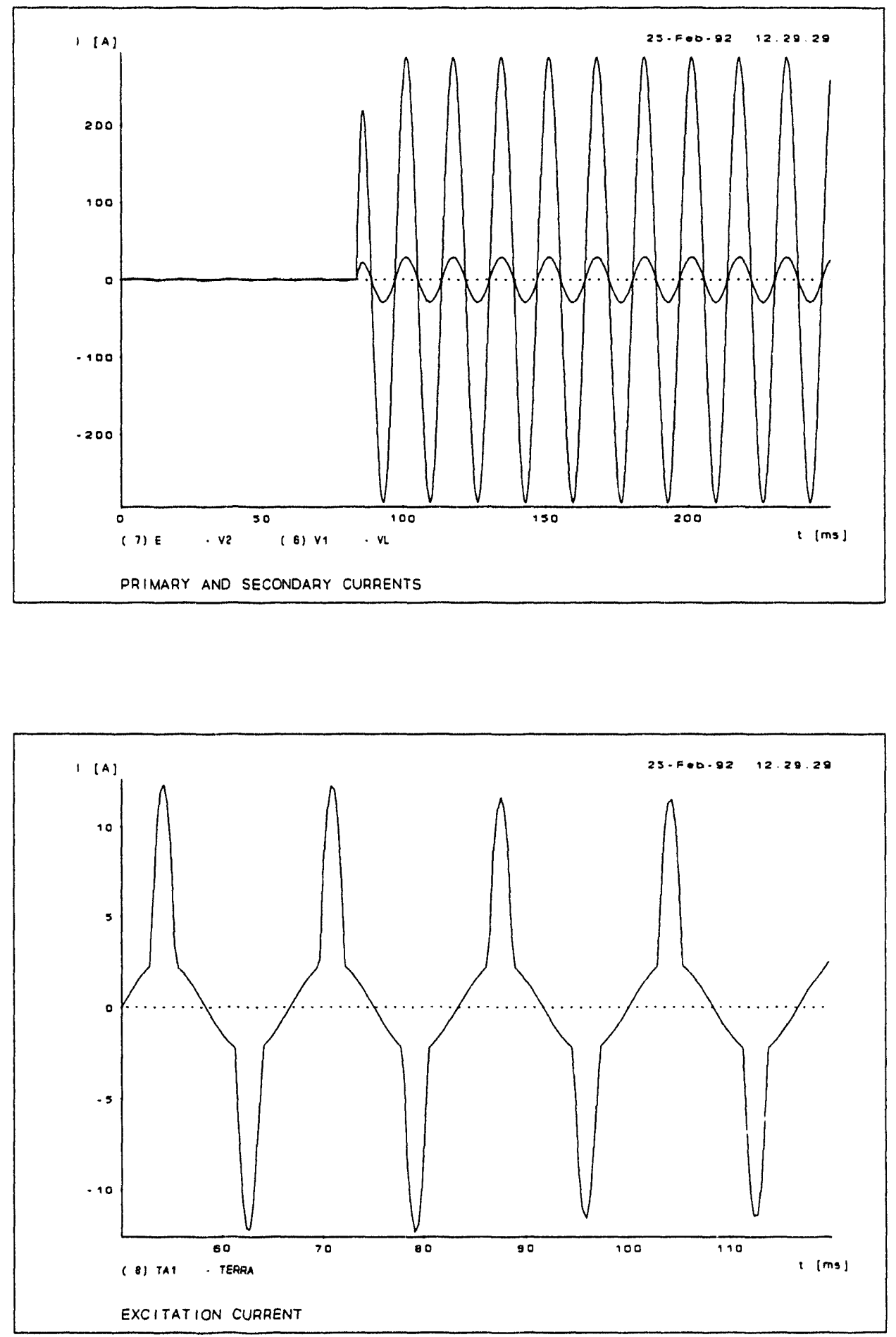

Fig. C-4. Case 1: Initially excited transformer with load switched in after 5 cycles. 

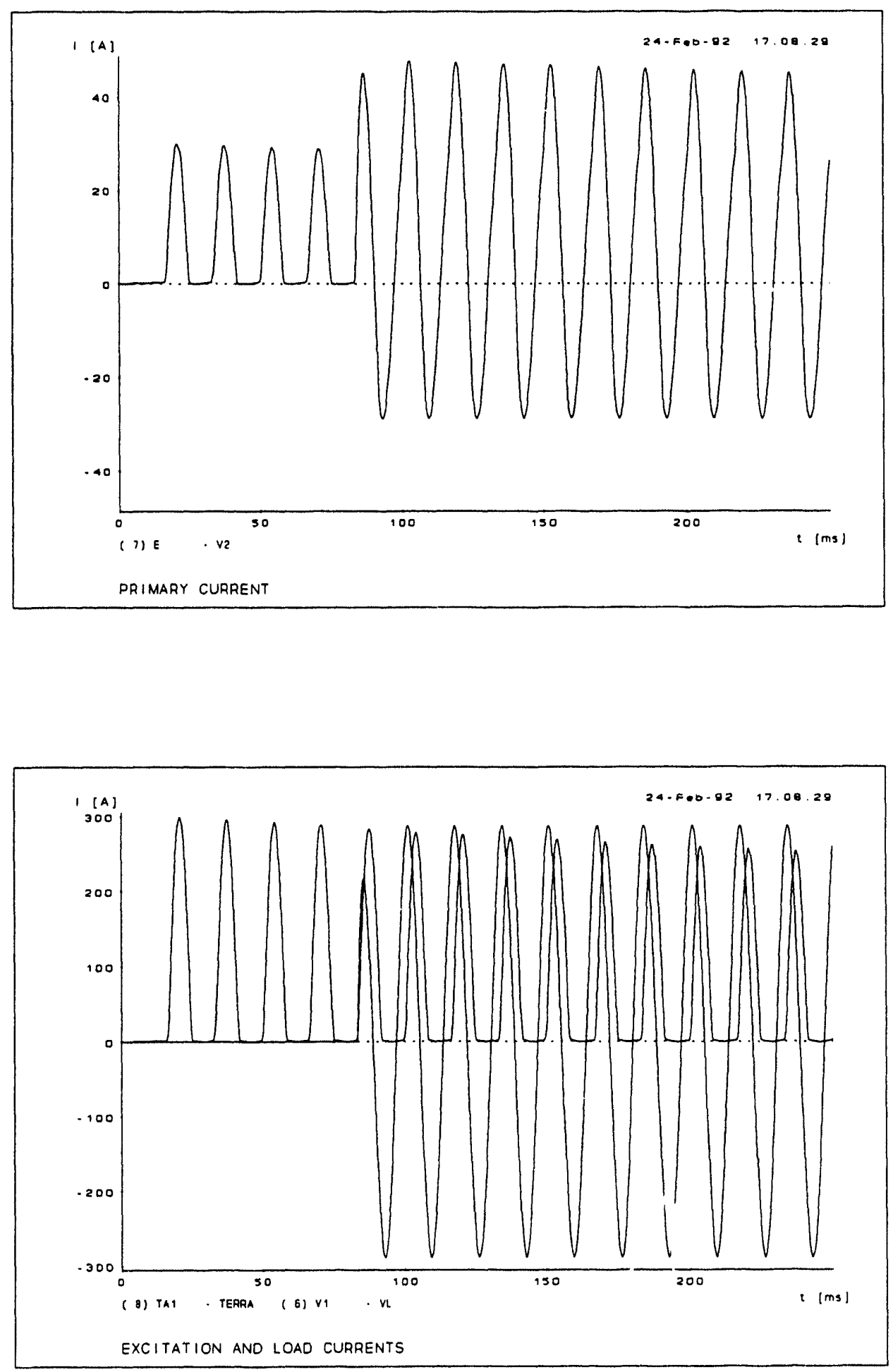

Fig. C-5. Case 2: inrush current simulation. 
CASE 3: This example shows the effect of including the hysteresis characteristic combined with saturation, for the same conditions as in the previous two cases.

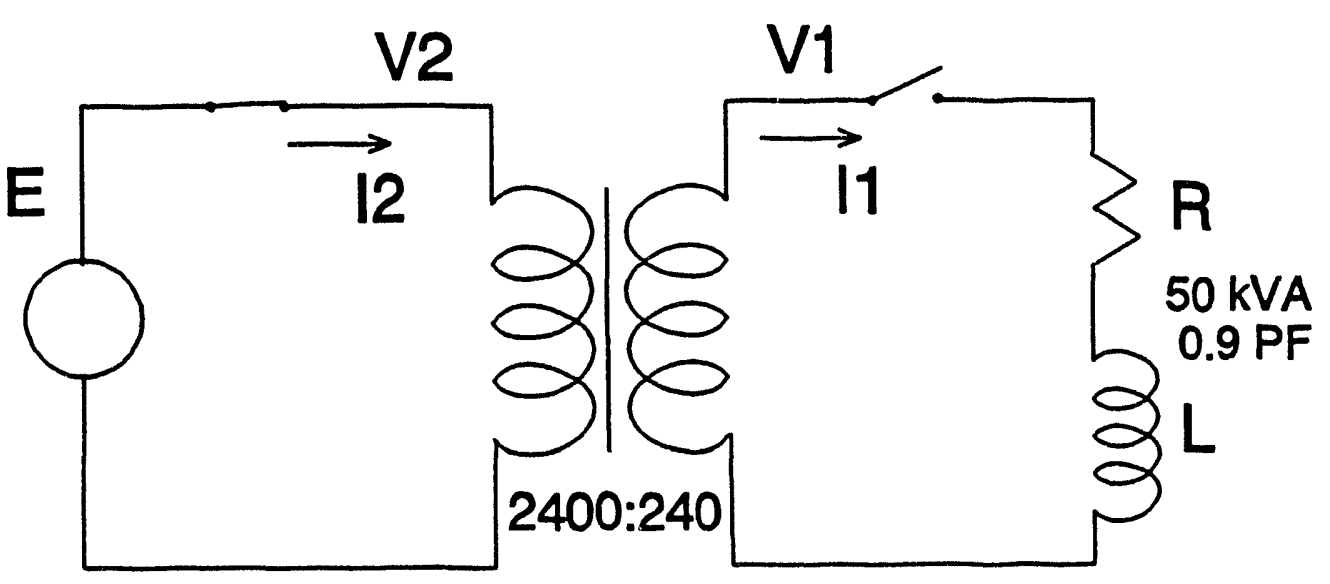

Fig. C-6

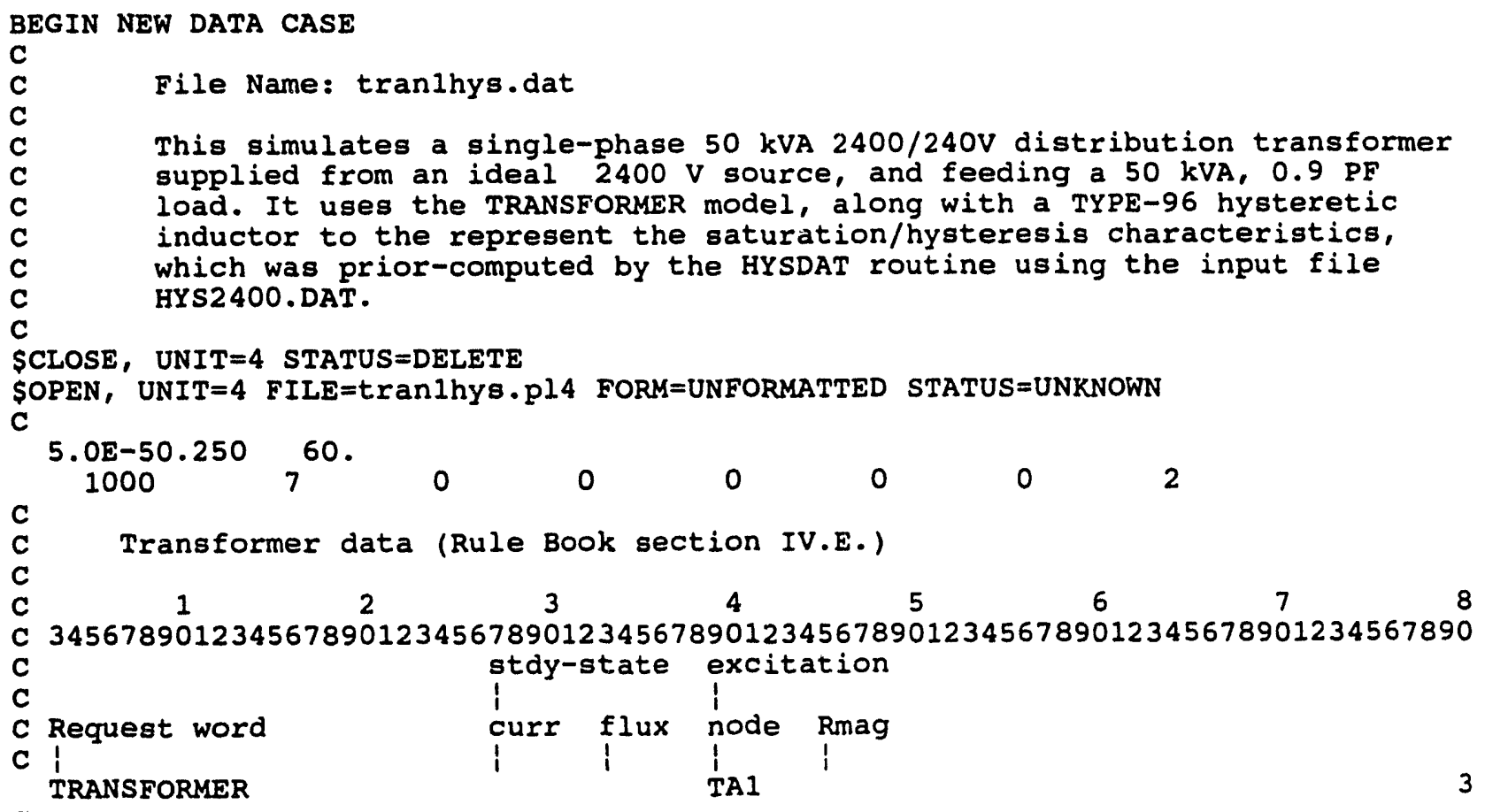

Magnetizing data (saturation characteristics) omitted, to be replaced by TYPE-96 hysteretic inductor.

9999

Winding data (resistance, leakage reactance, voltage rating)

Magnetizing branch always connected to winding 1 (low voltage)

$\begin{array}{lll}R 1 & X 1 & V R I \\ i & ! & 1 \\ .0 & .00001 .240\end{array}$ 


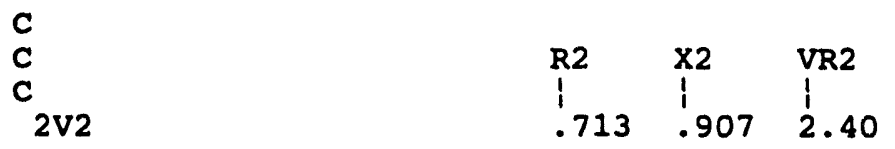

$\begin{array}{lcc}\mathrm{C} & & \\ \mathrm{C} & \text { Type-96 hysteretic inductor to represent magnetizing branch } \\ \mathrm{C} & \\ 96 \mathrm{~V} 1 & 8888 .\end{array}$

3

\$INCLUDE, hys2400.pch, \{ hysteresis data from previously computed file

C Transformer low voltage winding resistance and leakage reactance

C

V1 VT1 $\quad .00713 .00906$

C

C Load Data

C

VI

$1.037 \cdot 5021$

BLANK END OF BRANCH DEFINITIONS

C

C Energizing switch

VT1 VL .083333310.

C Measuring switch

C

E V2

MEASURING

1

C

BLANK END SWITCH CARDS

C

C Source data

C

$14 \mathrm{E}$

C

$3394.0 \quad 60.0$

0.0

$-.0001$

BLANK END OF SOURCE DEFINITIONS

C

C Node voltages for plotting

E V2

C

C FOURIER ON

C 1941.7 .4324 .1

C 1941.7 .4324 .1

C 1841.7 .4324 .1

C

BLANK CARD ENDING PLOT CARDS

BLANK END OF SIMULATION

BEGIN NEW DATA CASE

BLANK END OF ALL CASES

\section{Modification of switch cards for simulation of inrush currents:}

C

C Energizing switch

C

V1 VL $.0833333 \quad 10$.

C Magnetizing switch

C

$\begin{array}{llll}E & \text { V2 } & .0125 & 10 .\end{array}$

C

BLANK END SWITCH CARDS 

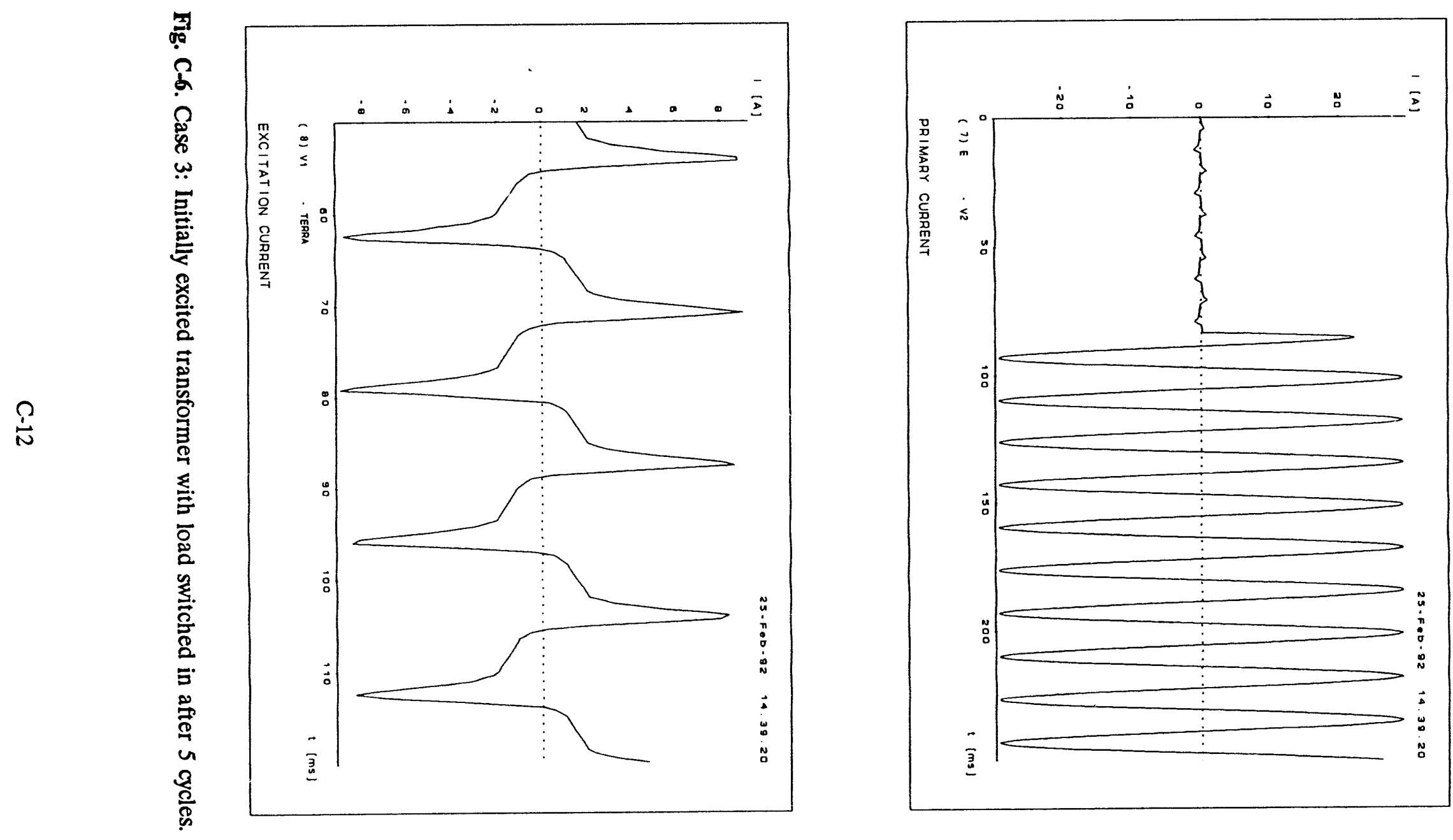

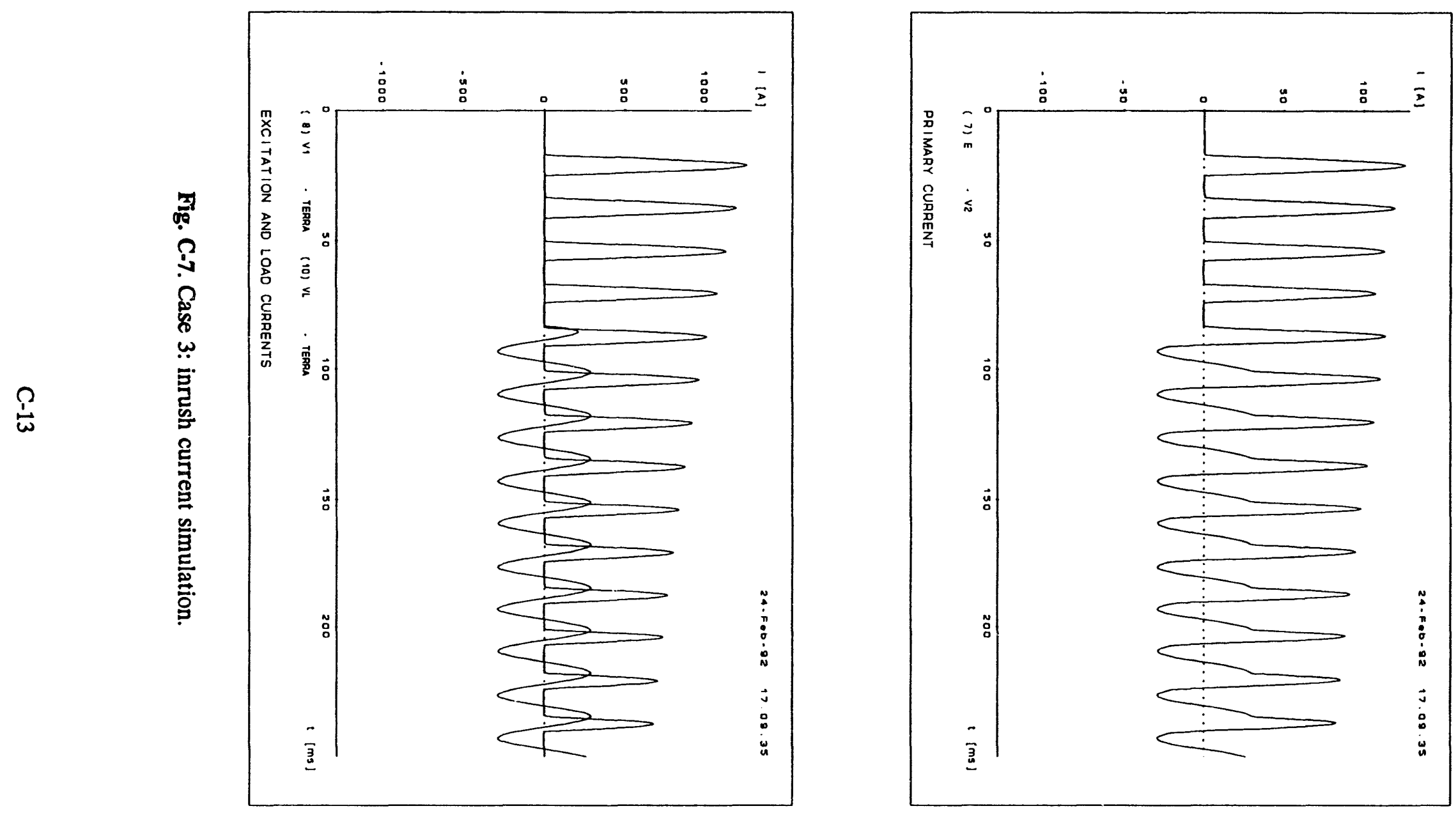


\section{C.3 GENERATOR AND POWER SYSTEM INTERACTIONS}

In some investigations it is necessary to represent the generator in detail, where the effects of the individual rotor and stator windings, the excitation system, and the prime-mover dynamics are important. EMTP has two different models available for this type of detailed synchronous machine representation: the TYPE 59 model and the UM (Universal Machine) model. The following example illustrates the use of the TYPE 59 model to simulate the behavior of a 4-pole nuclear unit during a severe disturbance in the surrounding network. It also shows how the TACS (Transient Analysis of Control Systems) routine is used to represent the various blocks and transfer functions contained in the generator excitation system model and the mechanical prime-mover/governor system model. TACS also is used to represent the complicated time-dependent frequency and magnitude characteristics of the remote systems, which was recorded durimg the disturbance. The three-line transmission network is modeled using equivalent PI sections, and the remote sources are represented by TACS-controlled sinusoidal voltage sources. The generator step-up transformer is also represented in three-phase detail using the TRANSFORMER model.

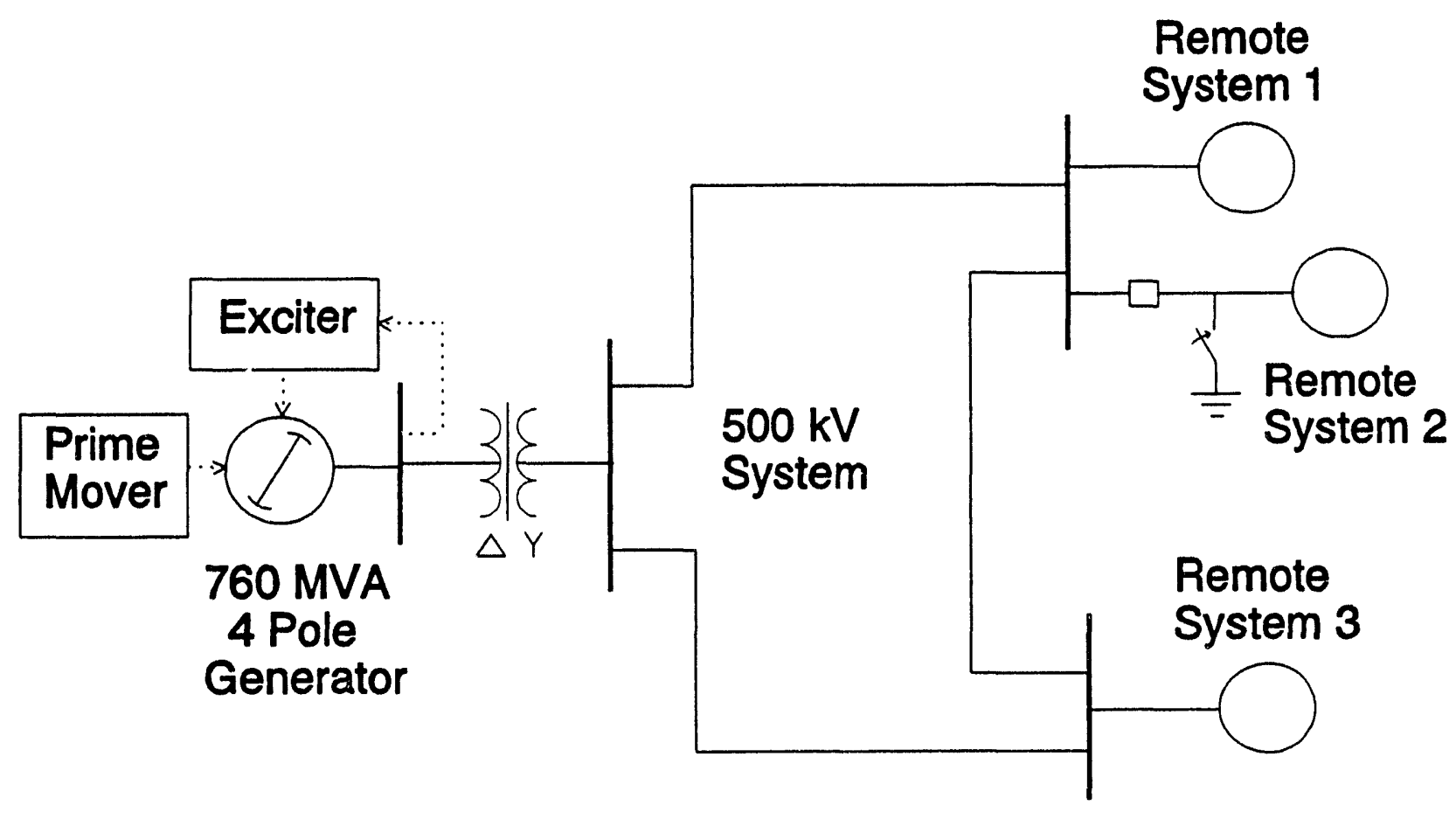

GENERATOR TRANSIENTS STUDY

Fig. C-8. System model for generator and system interaction example. 


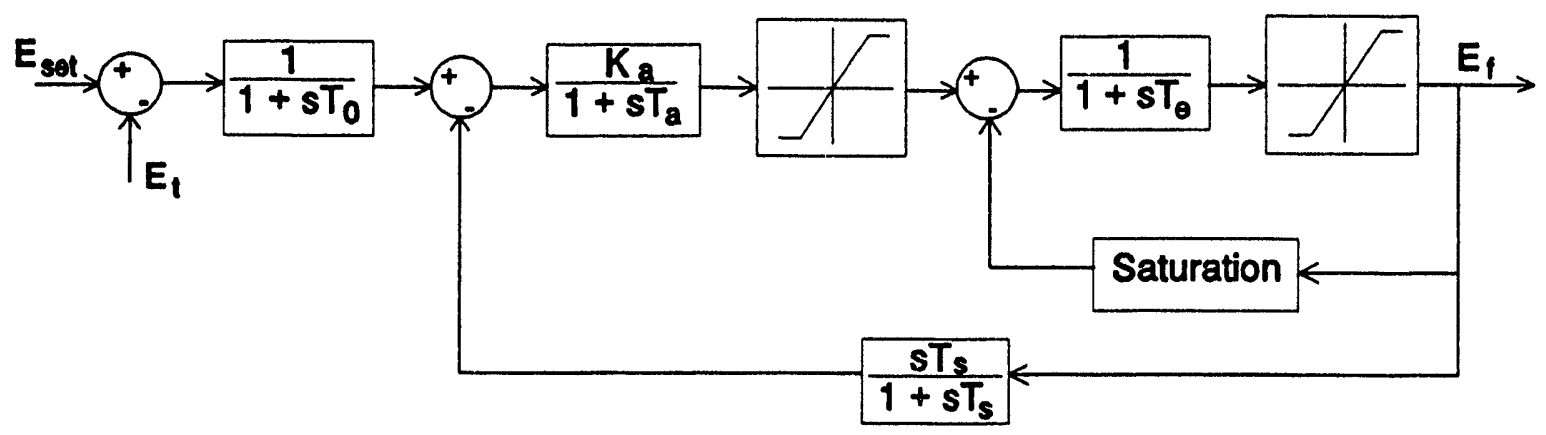

EXCITATION SYSTEM

Fig. C-9. Block diagram of generator excitation system model.

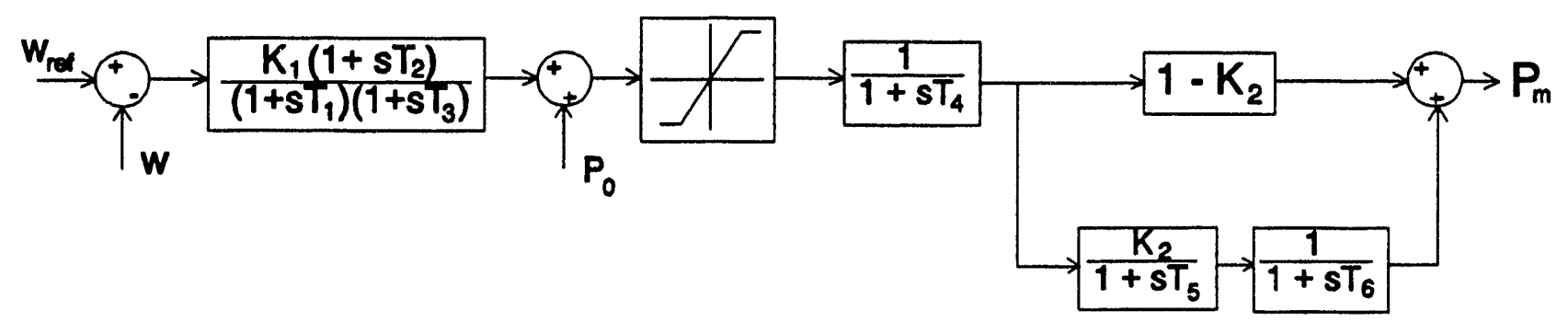

PRIME MOVER SYSTEM

Fig. C-10. Block diagram of prime-mover and governor system model. 


\section{C.3.1 EMTP input data file for type-59 generator transient study}

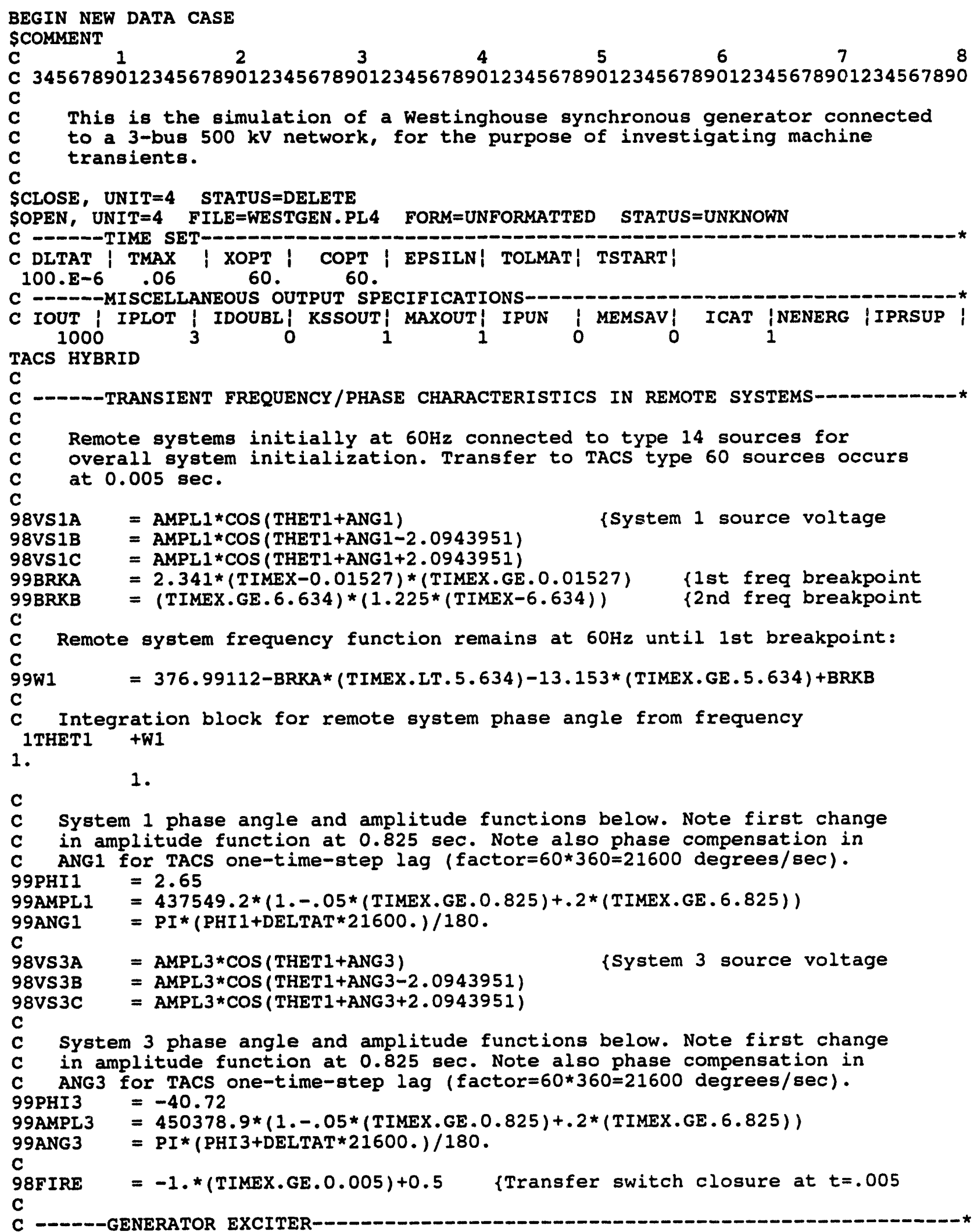


C

\begin{tabular}{|c|c|c|}
\hline EPS & +ETO & $-E M$ \\
\hline 1ERR & +EPS & \\
\hline 1. & .005 & \\
\hline EPR & +ERR & -EFB \\
\hline $\begin{array}{l}1 E F 1 \\
100 .\end{array}$ & $+E P R$ & \\
\hline 1 iEF2 & $\begin{array}{l}.03 \\
\text { +EF1 }\end{array}$ & \\
\hline $\begin{array}{l}1 . \\
88 \mathrm{ESAT} \\
\mathrm{C}\end{array}$ & $\begin{array}{l}.001 \\
=.01466\end{array}$ & $6 \star \operatorname{EXP}(1.206 * E F)$ \\
\hline EF3 & $+E F 2$ & -ESAT \\
\hline 1 1EF4 & +EF3 & \\
\hline 1. & .918 & \\
\hline$E F$ & +EF4 & \\
\hline 1EFB & $\begin{array}{l}+E F \\
: 1\end{array}$ & \\
\hline
\end{tabular}

\{zero-order block for voltage error signal

ffirst-order block for input error filter \{unity gain

\{time const $T O=.005$

\{zero-order block for feedback damping loop

ffirst-order block for main regulator

\{gain $\mathrm{Ka}=100$.

\{time const $\mathrm{Ta}=.03$

\{limiter block for $\max / \mathrm{min}$ regulator output

fexciter saturation function

\{zero-order block for saturation feedback

\{first-order block for exciter model

funity gain

\{time const $\mathrm{Te}=.918$

Exciter output limiter below:

\{first-order block for feedback damping loop \{gain $\mathrm{Ts}=1$.

$\{$ time const $T s=1$.

Note that output EF is per-unitized by scaling controller inputs, with initial ss output $=1$. This is necessary for use with Type-59 SM, which requires the TACS excitation input to be a multiplicative factor for the initialized field voltage setting.

-D---PRIME MOVER SYSTEM-

88FREF $=188.49556$

$88 \mathrm{PO}=1.0$

\{mechanical refrence freq, 4-pole $\mathrm{m} / \mathrm{c}$

$\mathrm{C}$

DELF TFREF -OMEGA

\{reference power setting

$2 \mathrm{PC} \quad+\mathrm{DELF}$

$1 . \quad .03$

$1 . \quad .33$

.027

\{zero-order block for freq error signal

zero-order limiter block for $\mathrm{min} / \mathrm{max}$ power output

PL $\quad+P O \quad+P C \quad 0 . \quad 1.1$

$1 P 1+P L$

1 .

1 .

.25

\{first-order block for steam inlet model

funity gain

ftime const $\mathrm{T} 4$

zero-order block for HP turbine fraction of power output

P2 +P1

$.306\{1-\mathrm{K} 2$ pu power

$2 \mathrm{P3}$

0.694

1 .

$+\mathrm{P} 1$

6.3

1.8

\{second-order block for reheat/crossover LP turbine \{gain K2 = pu LP turbine power

C

\{2nd-order equiv time const for $T 5$ and $T 6$ 


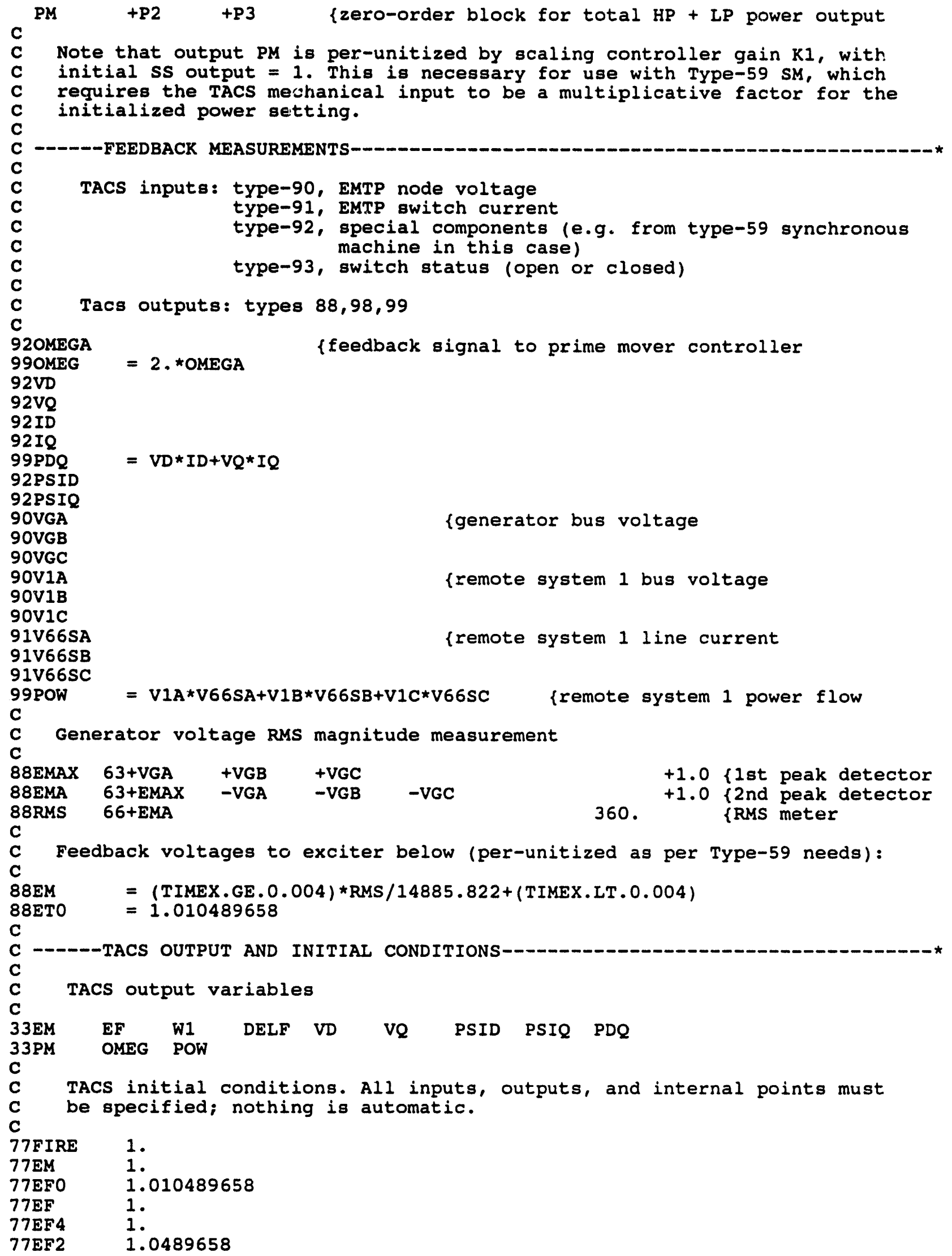




$\begin{array}{ll}\text { 77EF1 } & 1.0489658 \\ \text { 77EPS } & .010489658 \\ \text { 77EPR } & .010489658 \\ \text { 77ERR } & .010489658 \\ \text { 77OMEGA } & 188.49556 \\ \text { 77PL } & 1 . \\ \text { 77P1 } & 1 . \\ \text { 77P2 } & .306 \\ \text { 77P3 } & .694 \\ \text { 77PM } & 1.09 \\ \text { 77W1 } & 376.99112\end{array}$

BLANK FOR END OF TACS DATA C C C MISCELLANEOUS CIRCUIT CONNECTIONS vS1A VS1B VS1C VTIA VT1B VT1C VTIA VT1B VTIC VS3A VS3B VS3C VT3A VT3B VT3C VT3A VT3B c

10.

486.86 


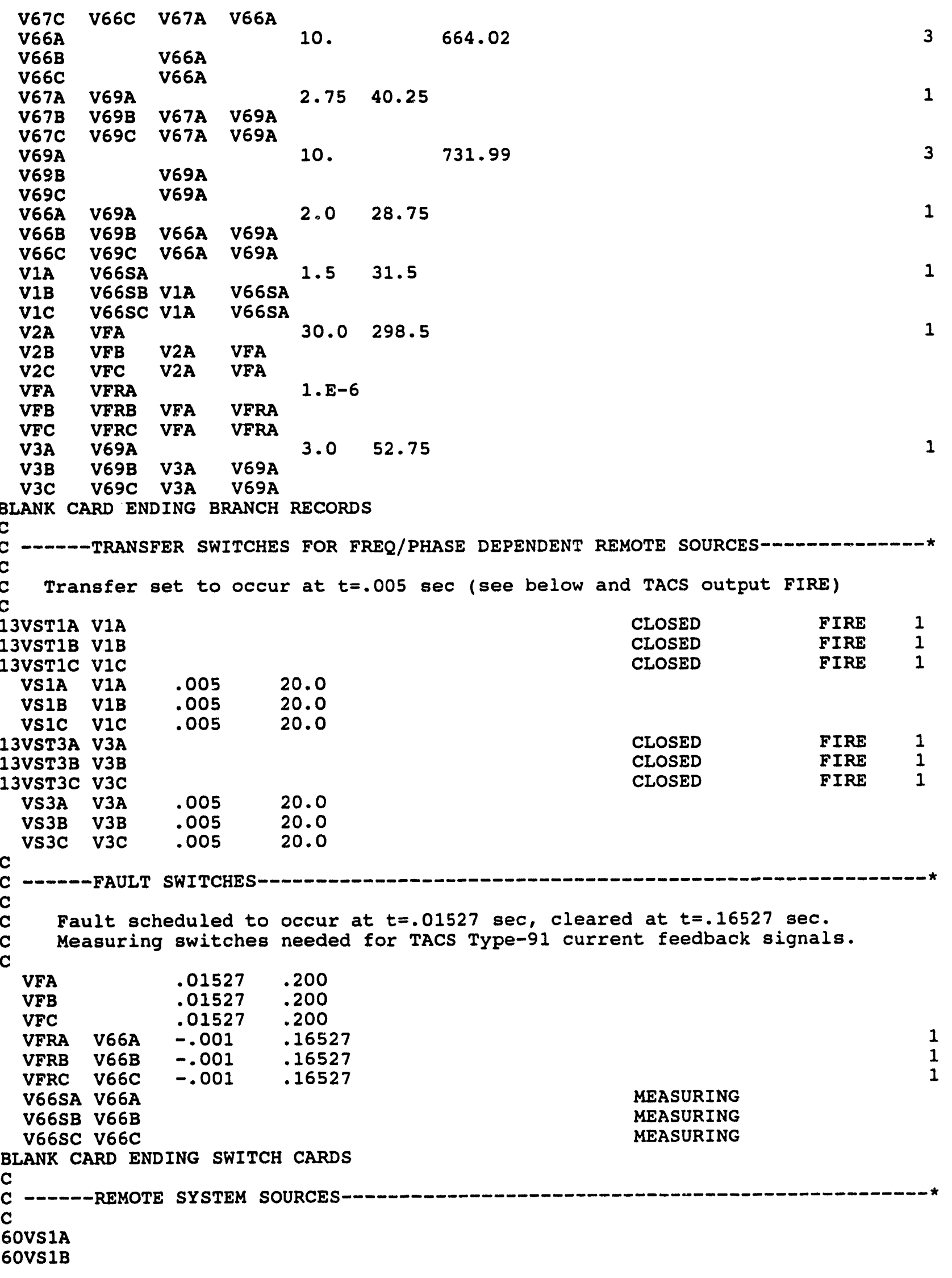


60Vs1C

60VS3A

60VS3B

60vs3c

C

C Type 60 TACs functions initially identical in magnitude and phase

C to corresponding Type-14 sources for smooth transition. System is

C initially connected to Type-14 sources for initializing purposes.

C

14VT1A

14VT1B

14VT1C

$14 \mathrm{~V} 2 \mathrm{~A}$

$14 \mathrm{~V} 2 \mathrm{~B}$

14V2C

14VT3A

$14 V T 3 B$

14VT3C

437549.260.

437549.260.

2.65

437549.260 .

$-117.35$

672674.960.

122.65

672674.960 .

$-80.8$ 672674.960 .

159.2 450378.960 .

$-40.72$

450378.960 .

$-160.72$

450378.960 .

79.28

-1
-1
-1
-1
-1
-1
-1
-1
-1

C

C

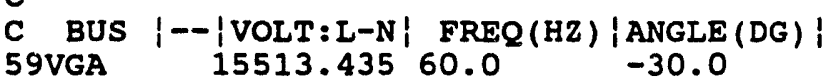

59VGB

59VGC

C - - - PARAMETER CARD TO ENTER STANDARD MACHINE DATA WITH FULL OPTIMIZATION PARAMETER FITTING 1.0

C

C - - - CLASS 3 S.M. DATA CARDS

C

C - - --THE FIRST SET SPECIFIES \#MASSES, MASS \# OF ROTOR AND OF EXCITER, AND

C - - THE NUMBER OF POLES OF THE SYSTEM, (COL 1-2,3-4,5-6, 7-10 RESPECTIVELY).

C ----SMOUTP/SMOUTQ IS FACTOR FOR SPLITTING POWER AMONG SEVERAL MACHINES.

C -- - RMVA, RKV ARE TOTAL 3-PHASE POWER RATING AND RATED L-I VOLTAGE (BASE).

C - - - AGLINE IS THE AMT OF FIELD I TO YIELD RATED VOLTAGE @ NO LOAD.

C - - - S1,S2 APPLY TO SATURATION MODELING.

C

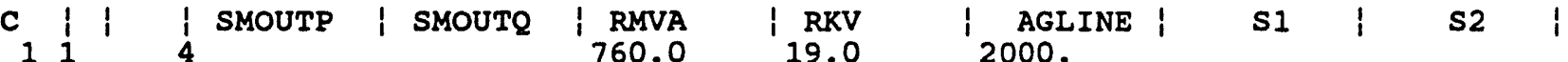
C

BLANK CARD (NO SATURATION MODELING)

C

C - - - THE NEXT 2 SETS REFER TO REMAINING MACHINE PARAMETERS AS SHOWN:

C

C

C

$\dot{\mathrm{C}}$

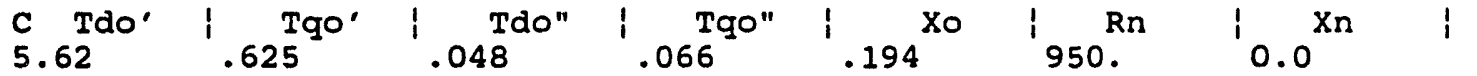

C

C

C

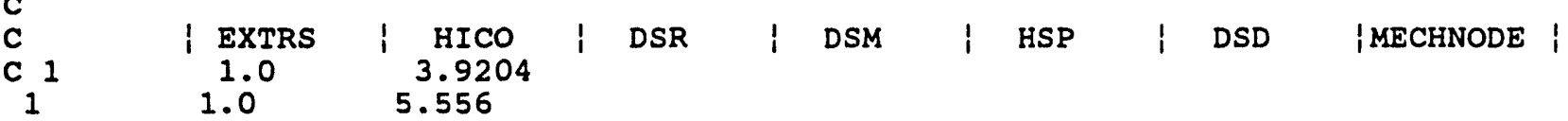

$=$

BLANR CARD ENDING MASS CARDS

C

C ----OUTPUT REQUESTS

51

11

21

31

BLANK RECORD ENDING OUTPUT REQUESTS FOR SM TYPE-59 ONLY 


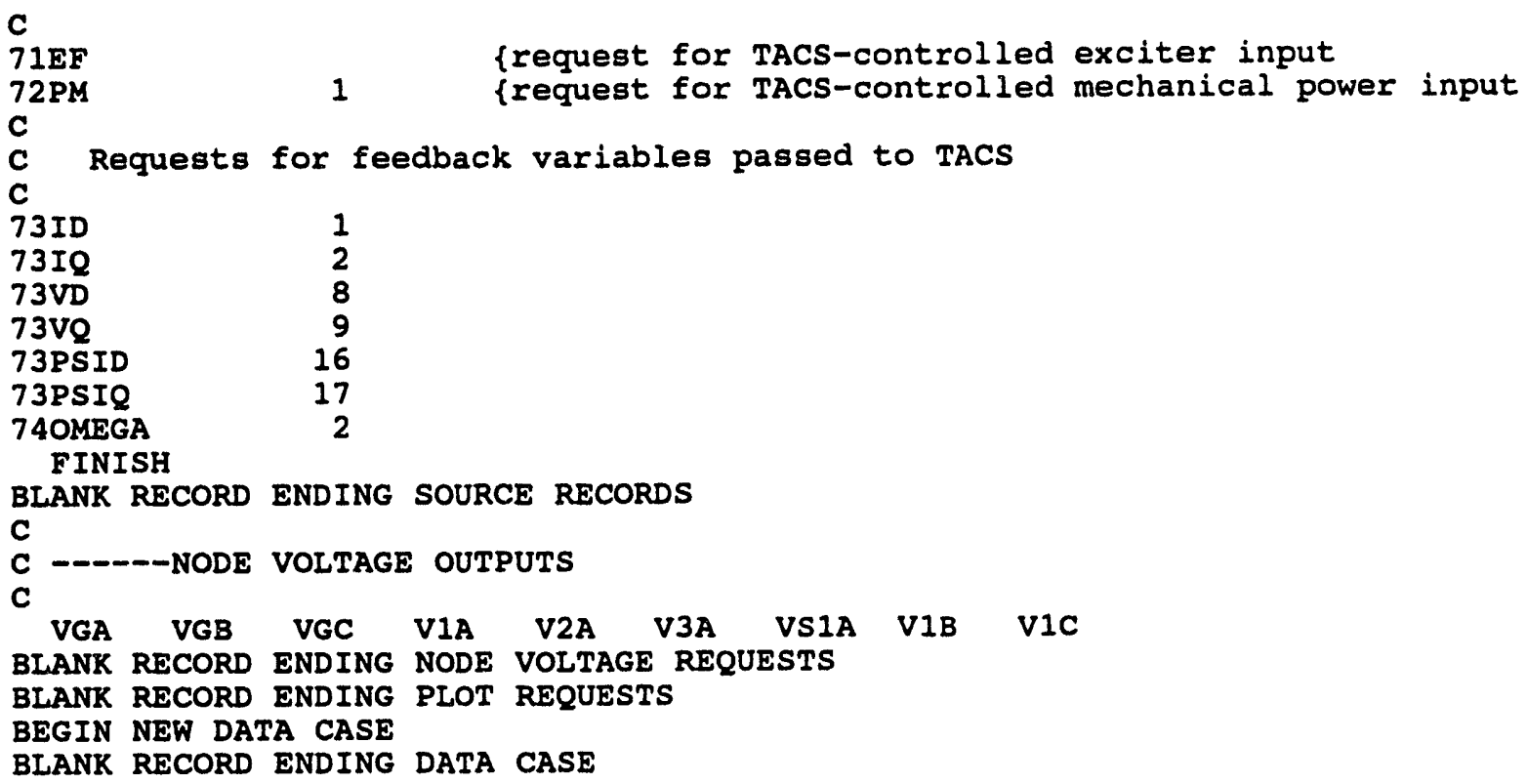

\section{C.3.2 Discussion of simulation results}

In this study the disturbance is modeled by a three-phase fault at remote system 1 , which occurs at approximately $15 \mathrm{msec}$. The fault is then cleared at approximately $165 \mathrm{msec}$. These two events are clearly evident in most of the following plots of machine variables. Another less obvious event that is also modeled in the simulation is a rapid decline of remote system frequency, accompanied by a time-changing magnitude and phase of the remote system voltages. This lasts well beyond the fault clearing time and thus effects the generator for several seconds. Figures A-11 and A-12 show the generator torque and output power during the disturbance. As expected, these outputs are depressed during the fault and then overshoot after clearing, due to rotor acceleration during the fault. Figures A-13 and A-14 show the generator angle and rotor speed deviation during the acceleration and deceleration periods. Figure A-14 reveals the continuously decreasing rotor speed after fault clearing, as the generator follows the declining frequency in the strong remote systems. Figures A-15 and A-16 show the generator stator voltage and current during the transient, revealing the persistent fault current with a large dc offset. Figures A-17 and A-18 show the generator field voltage and current during the disturbance. Figure 17 shows the induced current during the fault, and both plots show how the exciter attempts to raise the voltage to compensate for the fault. Not shown in these waveforms is the longer term effect of the time-changing frequency, magnitude and phase angles of the remote system sources. 


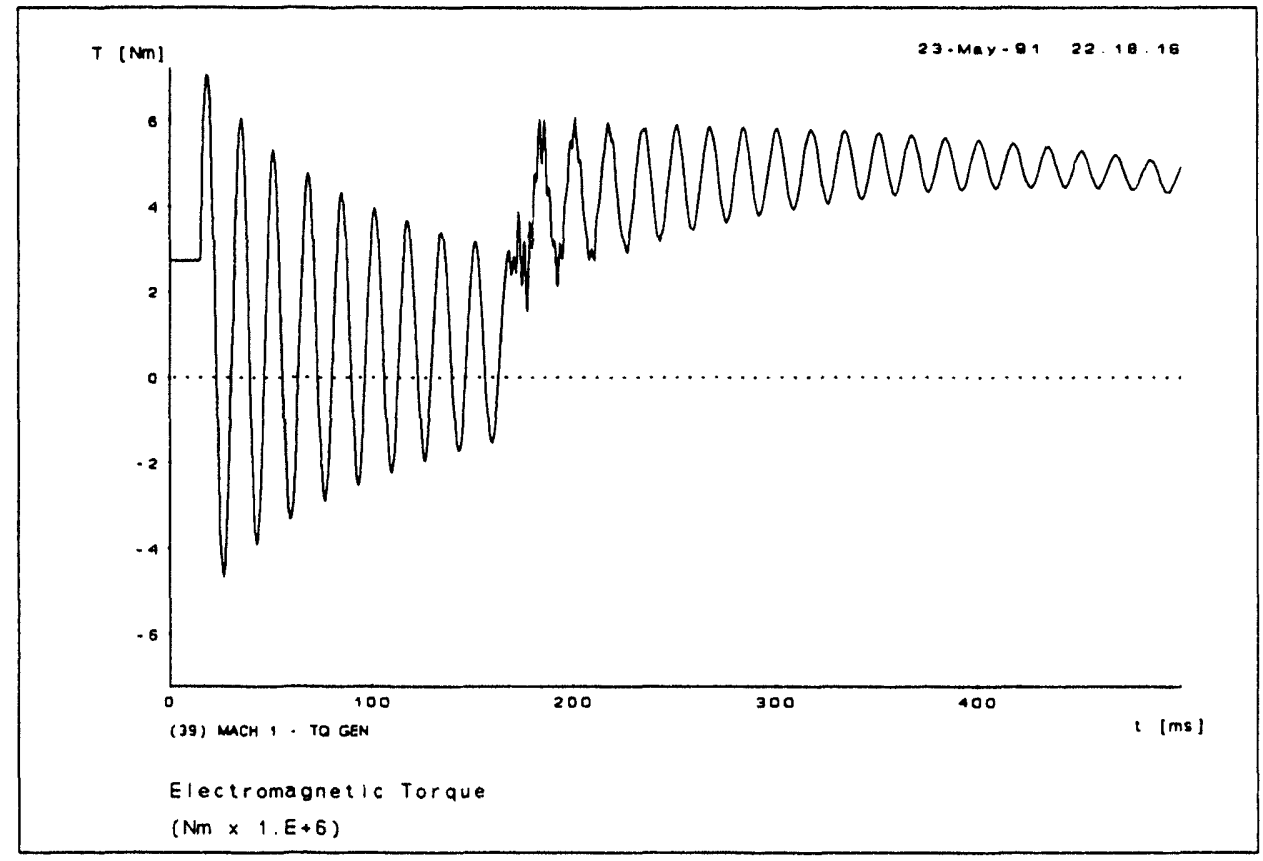

Fig. C-11. Generator electromagnetic torque during transient disturbance.

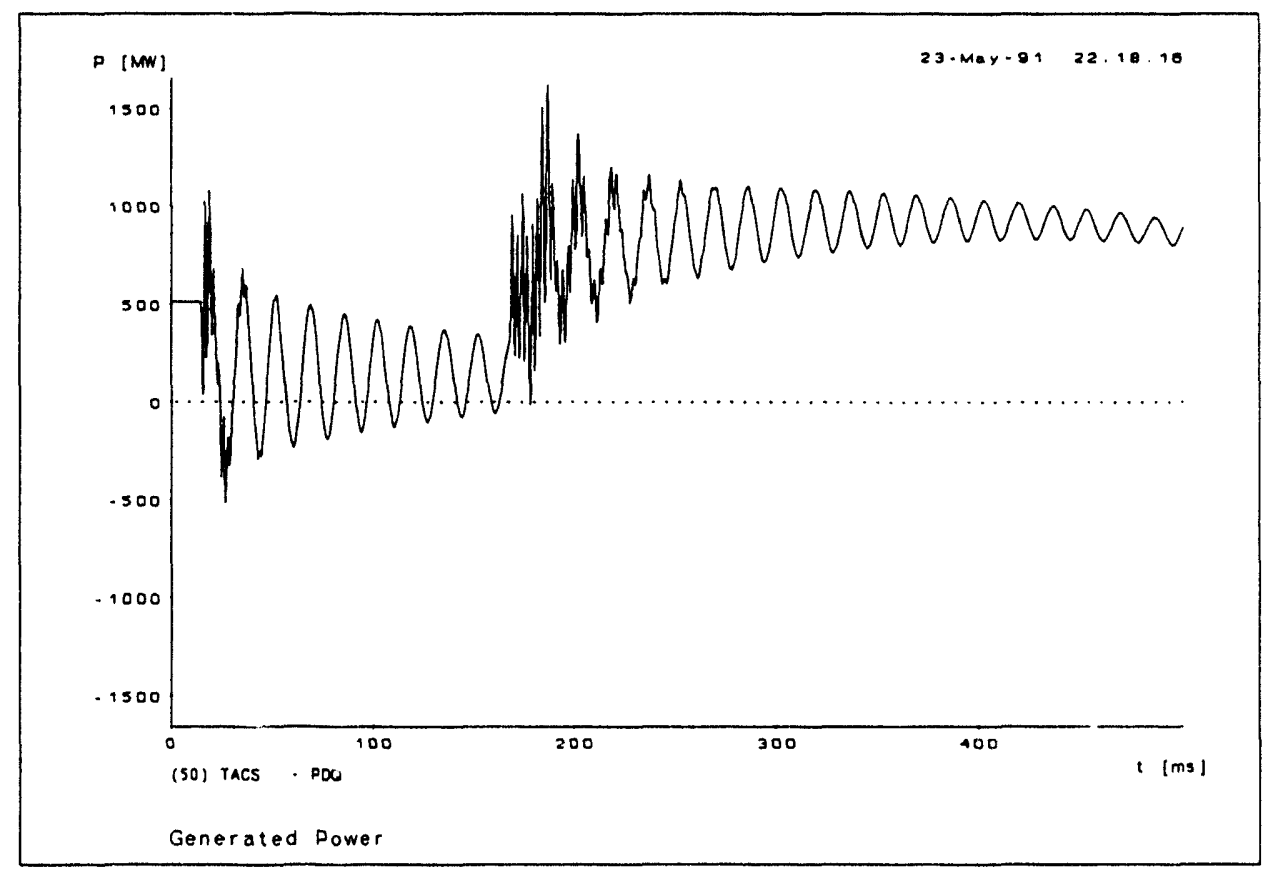

Fig. C-12. Generator power output during transient disturbance. 


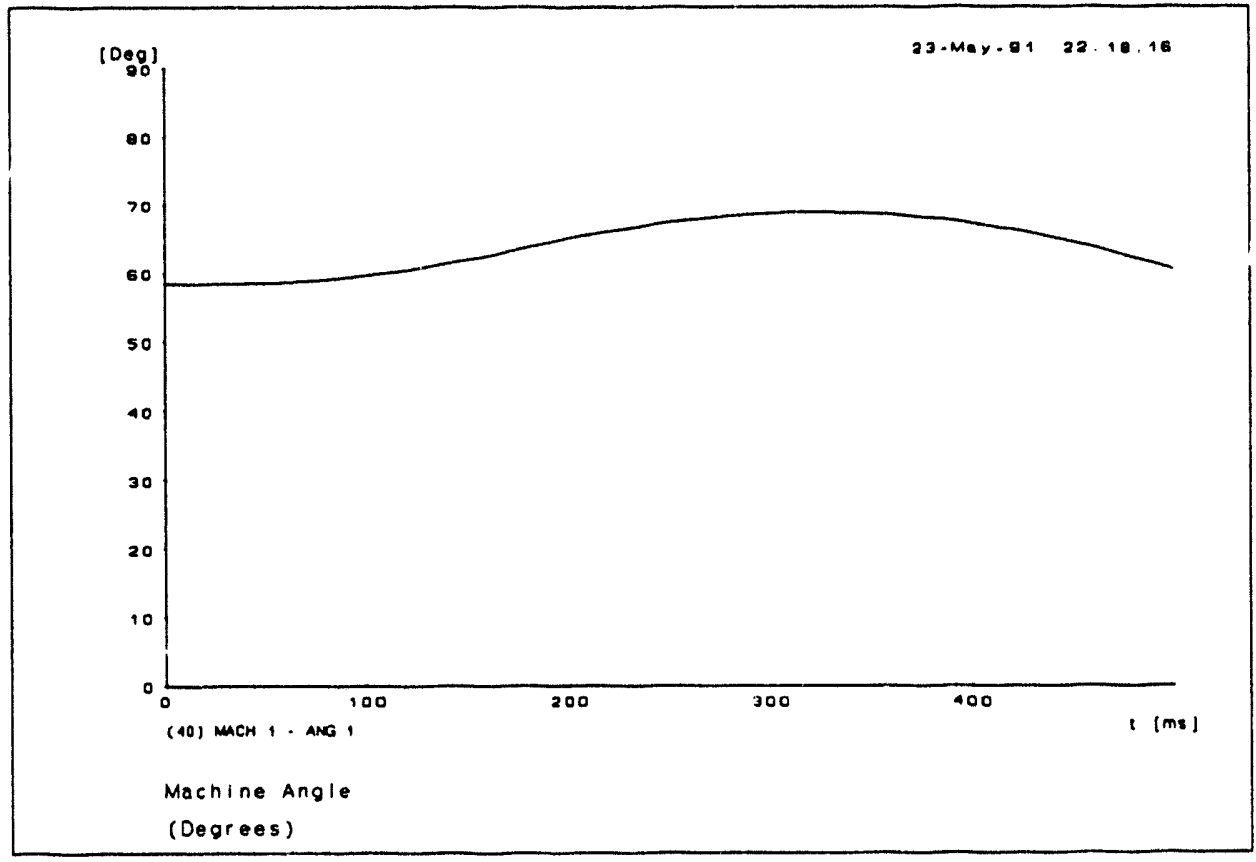

Fig. C-13. Generator angle during transient disturbance.

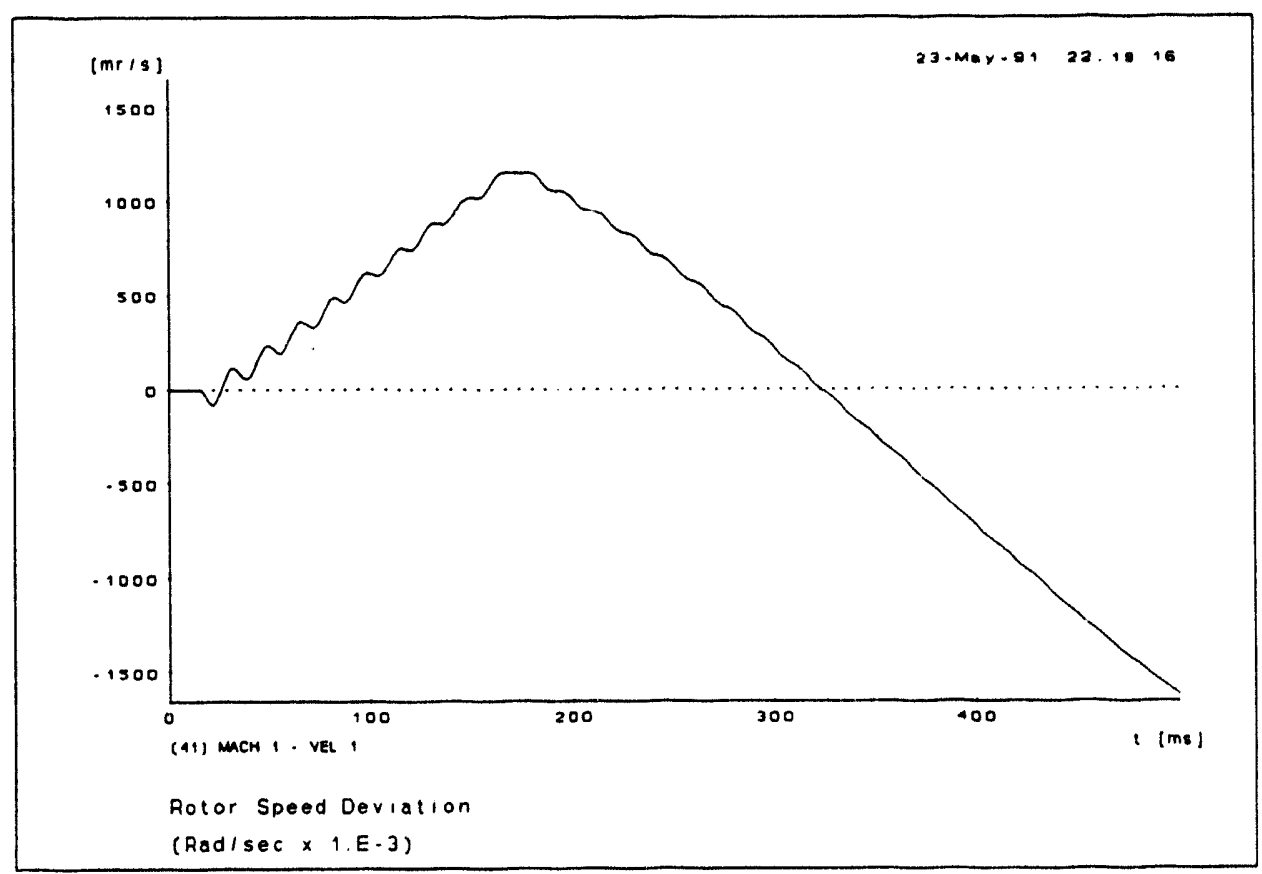

Fig. C-14. Rotor speed deviation from synchronous during transient distrubance. 


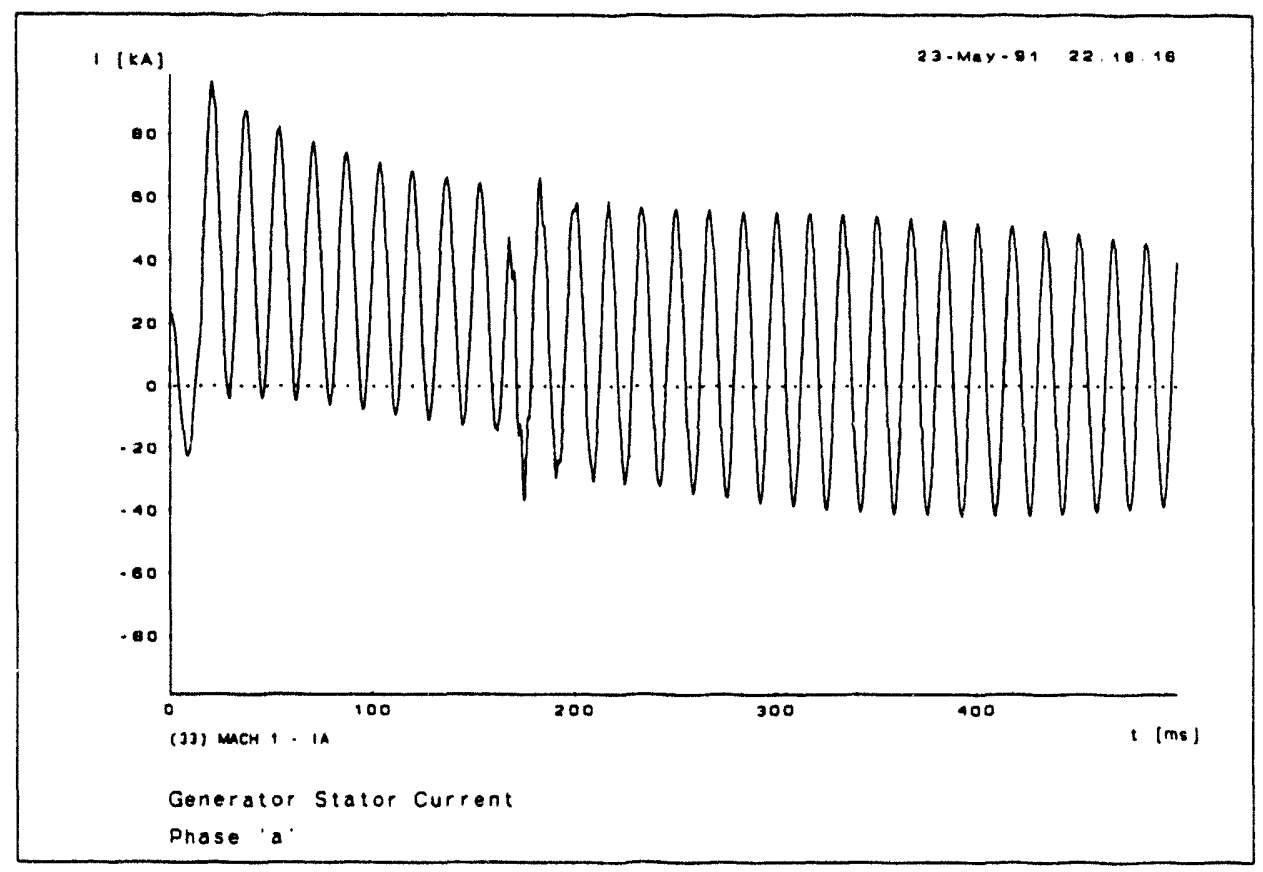

Fig. C-15. Generator stator current (phase A) during transient disturbance.

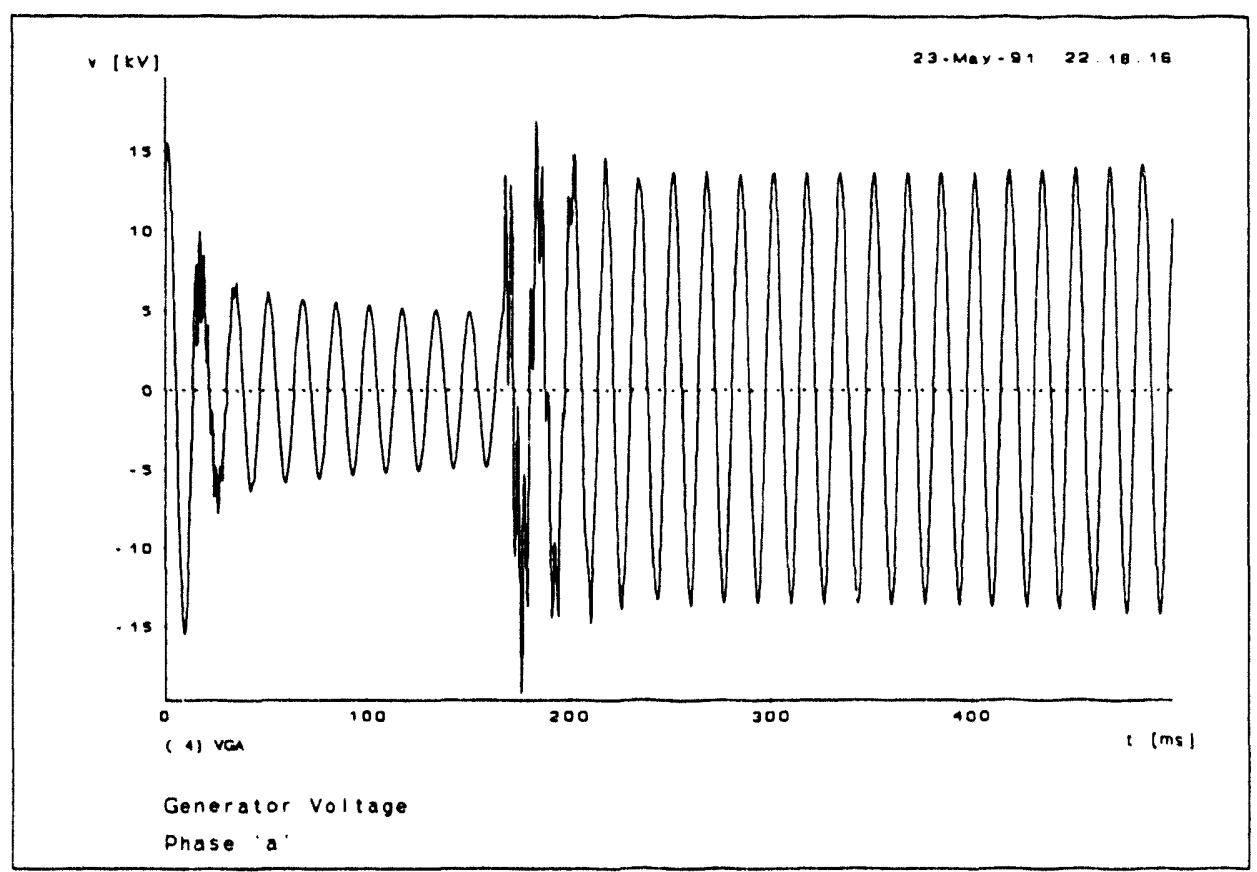

Fig. C-16. Generator terminal voltage (phase A) during transient disturbance. 


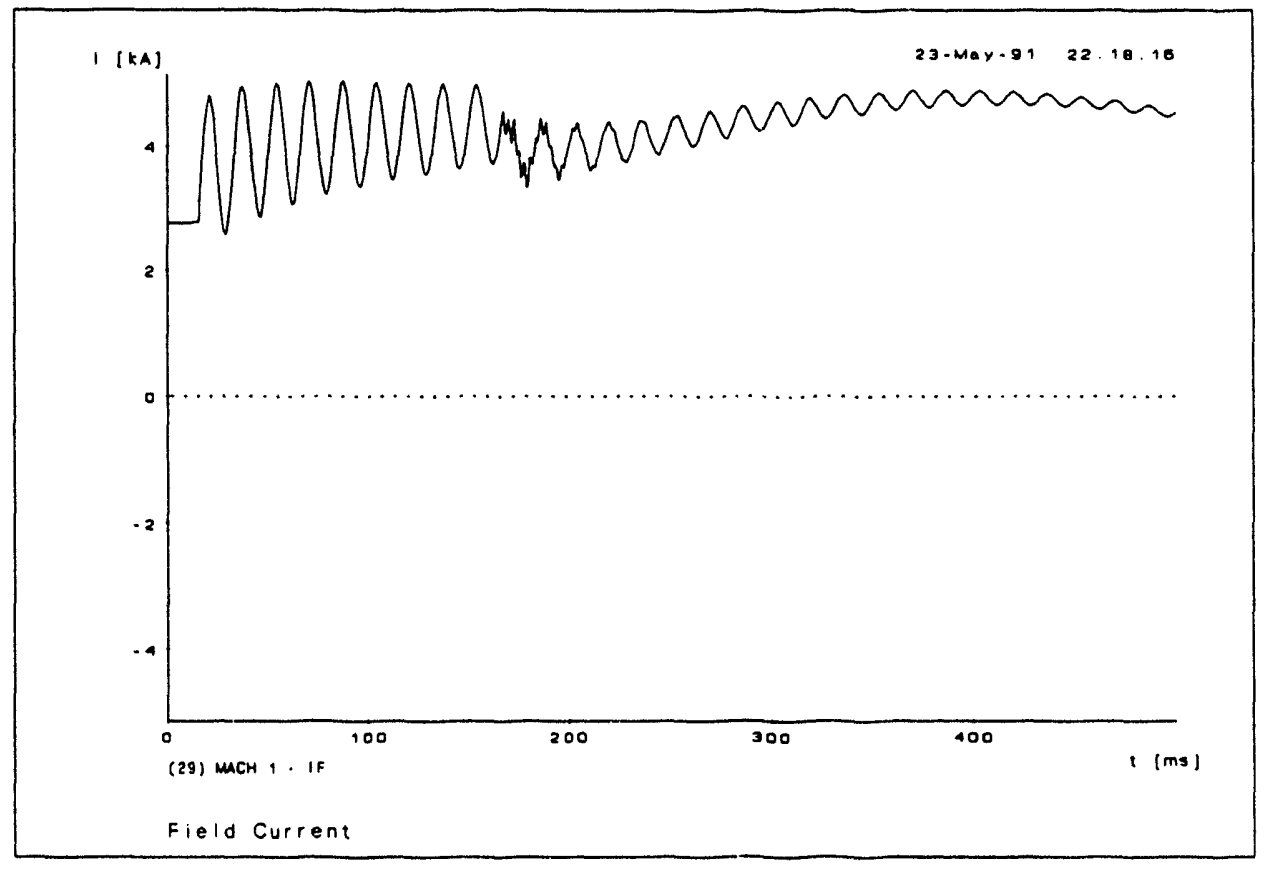

Fig. C-17. Generator field current during transient disturbance.

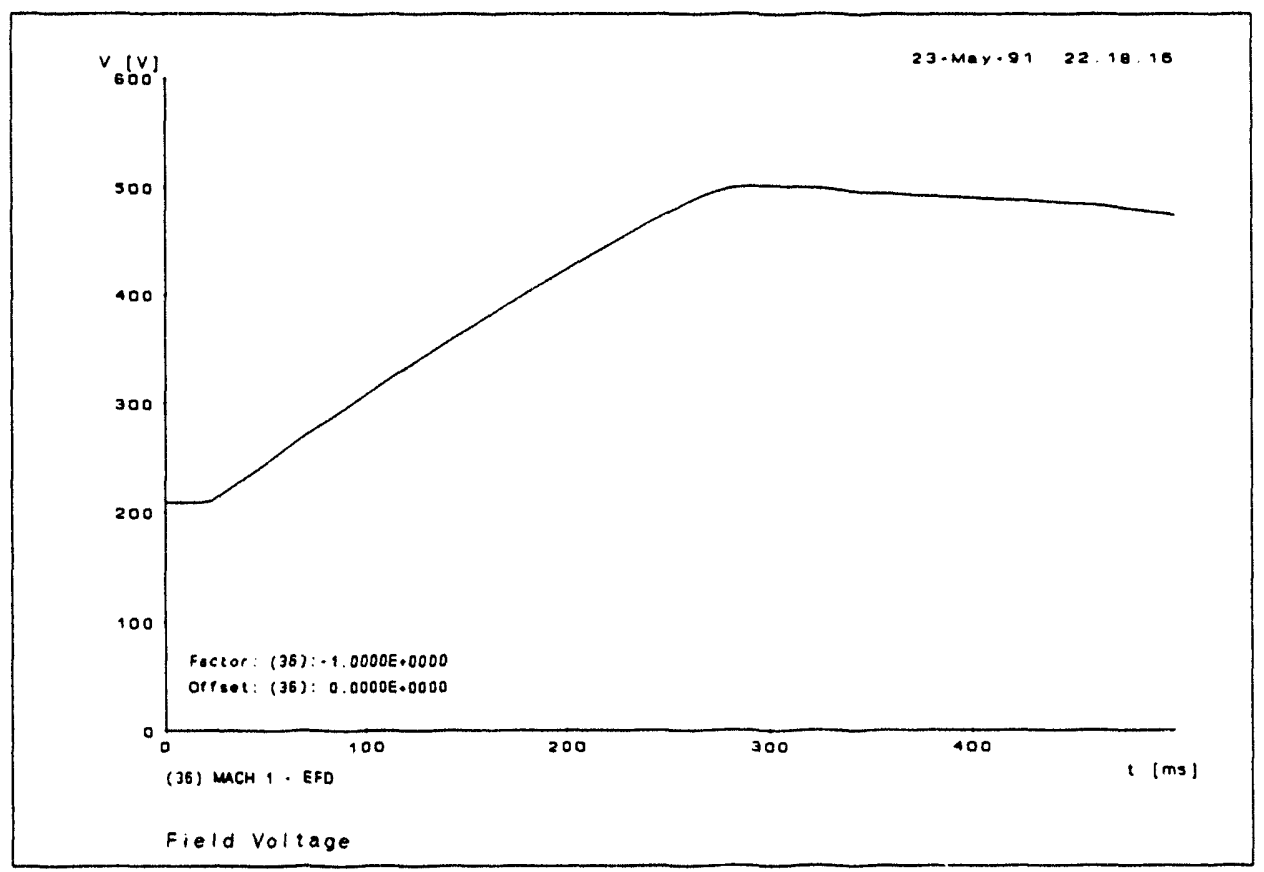

Fig. C-18. Generator field voltage during transient disturbance. 
APPENDIX D:

\section{SALEM 1 SIMULATION AND SAMPLE RESULTS}

\section{EMTP Data Input Files}

\section{D.1 MAIN PROGRAM}

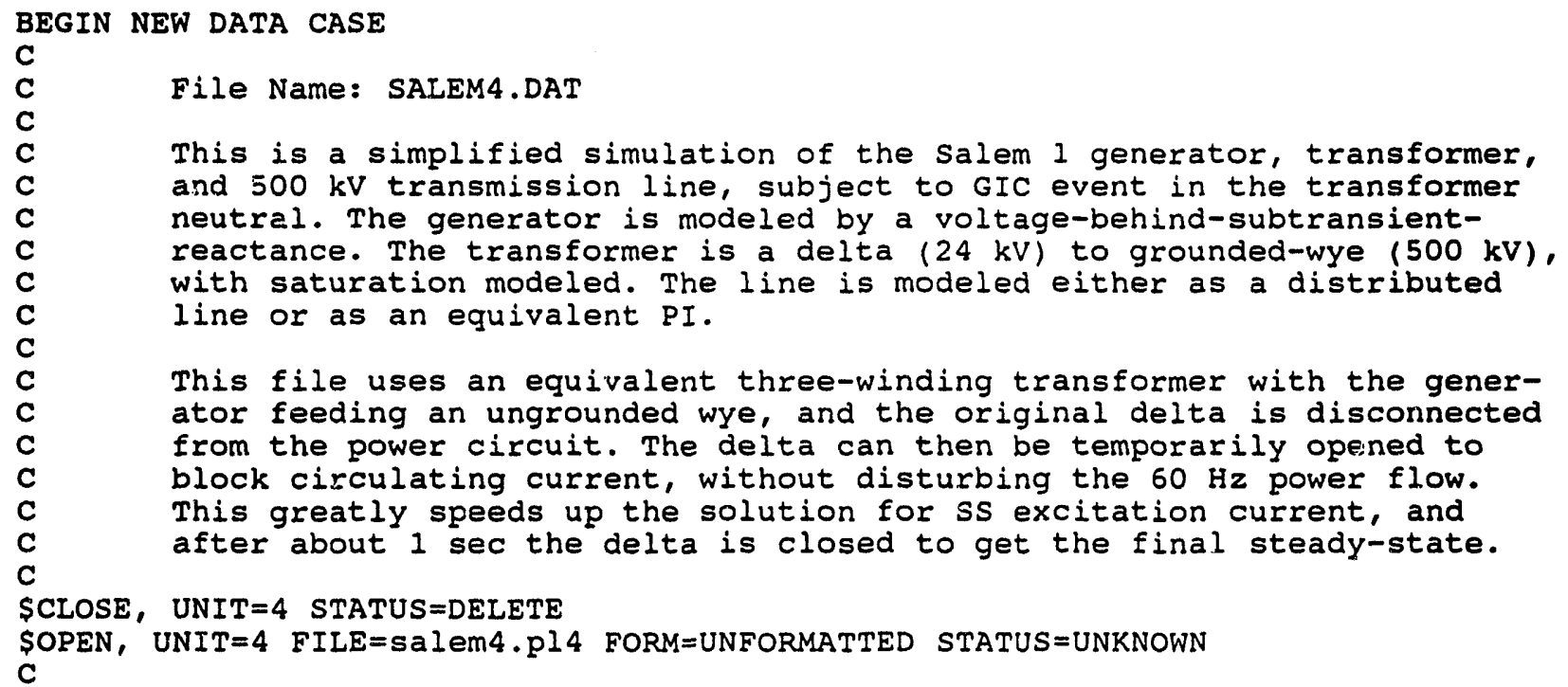

C

$5.0 E-52.060$. 1000

1500

$1 \quad-1$

0

2

C

C

C Request word

C I

Transformer data

TRANSFORMER

Magnetizing data

INCLUDE, sat $24 \mathrm{kv}$.inc,

C Winding data (resistance, leakage reactance, voltage rating)

C Magnetizing branch connected to winding 1 (low voltage)

C

C

ILOWA LOB

$\begin{array}{lll}R 1 & X 1 & \text { VRI } \\ 1 & 1 & 1\end{array}$

c

C

C

2HIGHA NEUT

3GENA FLOAT

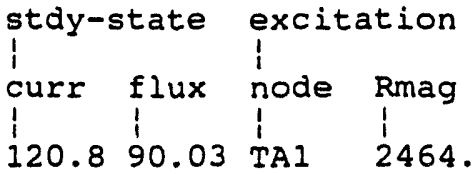

C

TRANSFORMER TAI

.00156 .129524 .0

1LOWB LOWC

2HIGHB NEUT

3GENB FLOAT

TRANSFORMER TA1

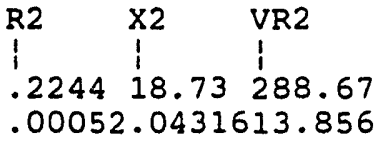

TBI 


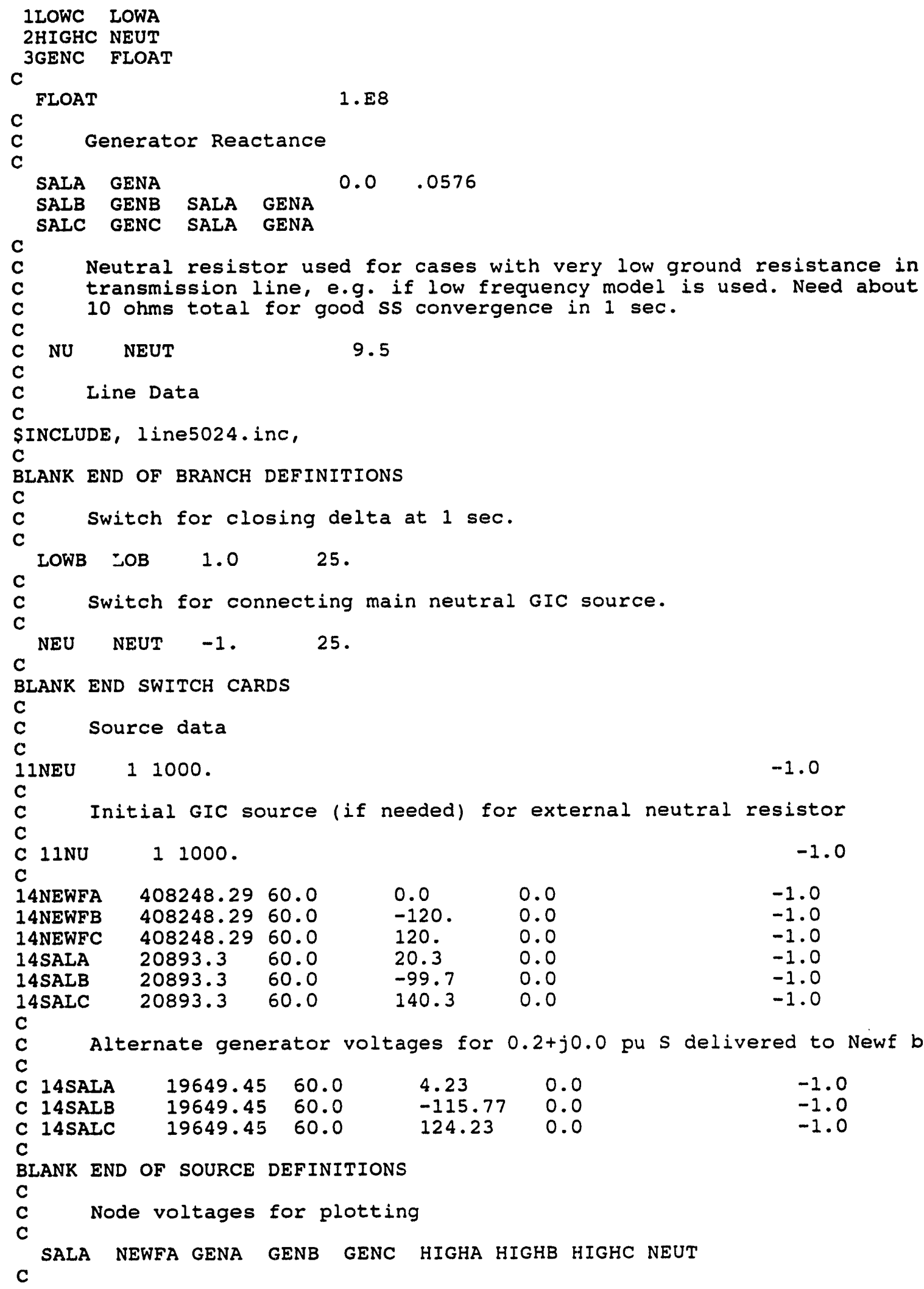

C BLANK END SWITCH CARDS

C

C Source data

C

11 NEU I 1000 .

c

c

C

C 11NU

Initial GIC source (if needed) for external neutral resistor

C

14NEWFA

14NEWFB

14NEWFC

14SALA

14SALB

14 SALC

c

c

C

C 14SALA 19649.45

C 14SALB 19649.45

C 14SALC

11000 .

$-1.0$

$\begin{array}{ll}408248.29 & 60.0 \\ 408248.29 & 60.0 \\ 408248.29 & 60.0 \\ 20893.3 & 60.0 \\ 20893.3 & 60.0 \\ 20893.3 & 60.0\end{array}$

0.0

-120 .

120.

20.3

$-99.7$

140.3

0.0

0.0

0.0

0.0

0.0

0.0

$-1.0$

$-1.0$

$-1.0$

$-1.0$

$-1.0$

$-1.0$

$-1.0$

C

BLANK END OF SOURCE DEFINITIONS

C

C Node voltages for plotting

C

SALA NEWFA GENA GENB GENC HIGHA HIGHB HIGHC NEUT

$\begin{array}{lll}4.23 & 0.0 & -1.0 \\ -115.77 & 0.0 & -1.0 \\ 124.23 & 0.0 & -1.0\end{array}$


BLANK CARD ENDING PLOT CARDS

BLANK END OF SIMULATION

BEGIN NEW DATACASE

BLANK END OF ALL CASES

\section{D.2 LINE CONSTANTS ROUTINE:}

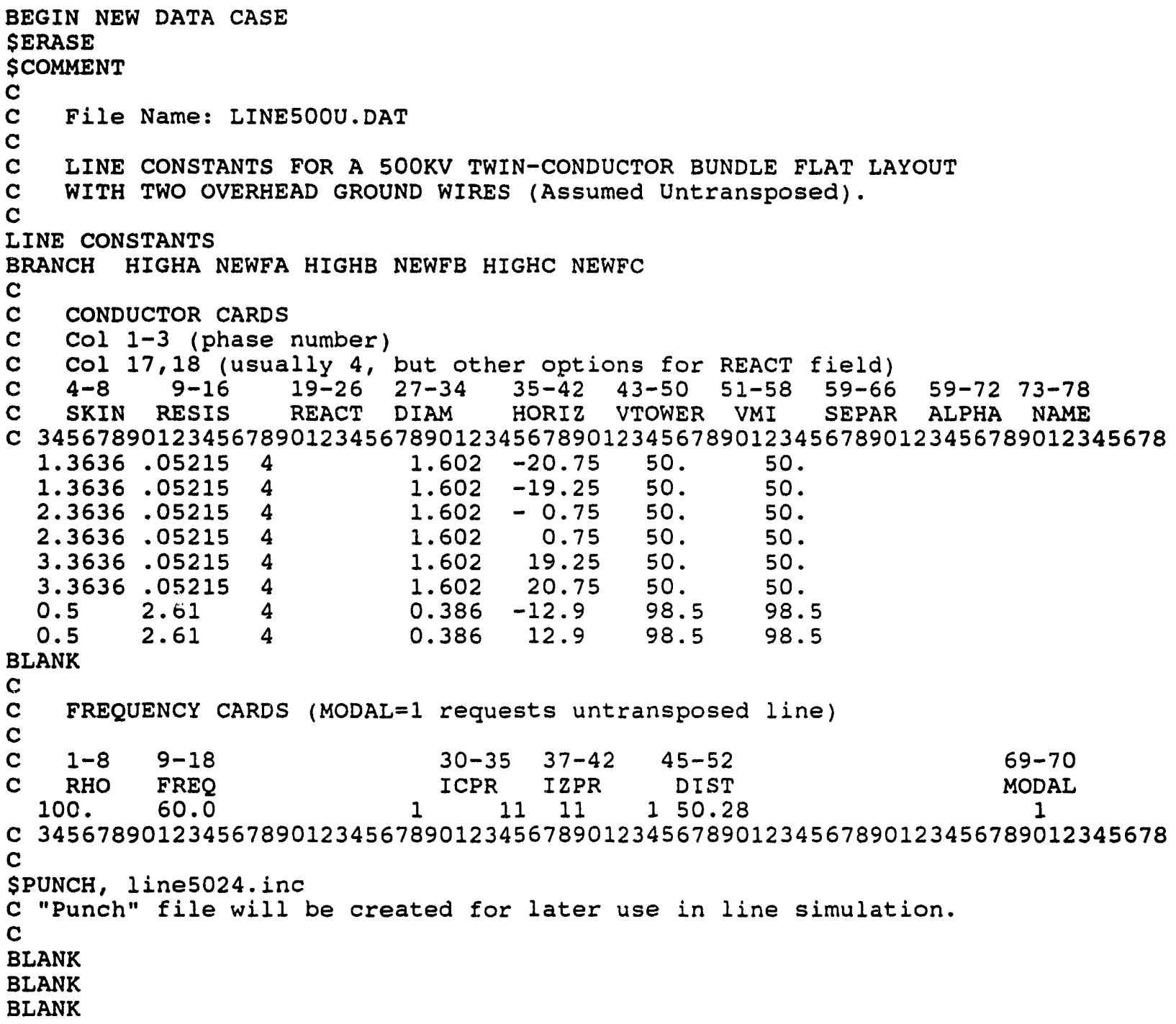

Distributed Iine Model Output Data:
\$VINTAGE, 1
- IHIGHA NEWFA

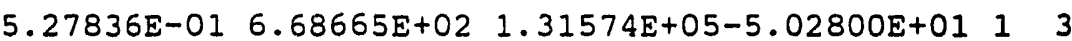
-2HIGHB NEWFB
$2.82200 E-022.90813 E+021.82761 E+05-5.02800 E+0113$
- 3 HIGHC NEWFC
$2.80261 E-02 \quad 2.37649 E+02 \quad 1.83704 E+05-5.02800 E+0113$ SVINTAGE, 0
$0.60449650-0.70710678-0.40908643$
$0.00000000 \quad 0.00000000 \quad 0.00000000$
$0.51881399 \quad 0.00000000 \quad 0.81565715$
$0.00000000 \quad 0.00000000 \quad 0.00000000$
$0.60449650 \quad 0.70710678-0.40908643$
$0.00000000 \quad 0.00000000 \quad 0.00000000$ 


\section{D.3 TRANSFORMER SATURATION ROUTINE:}

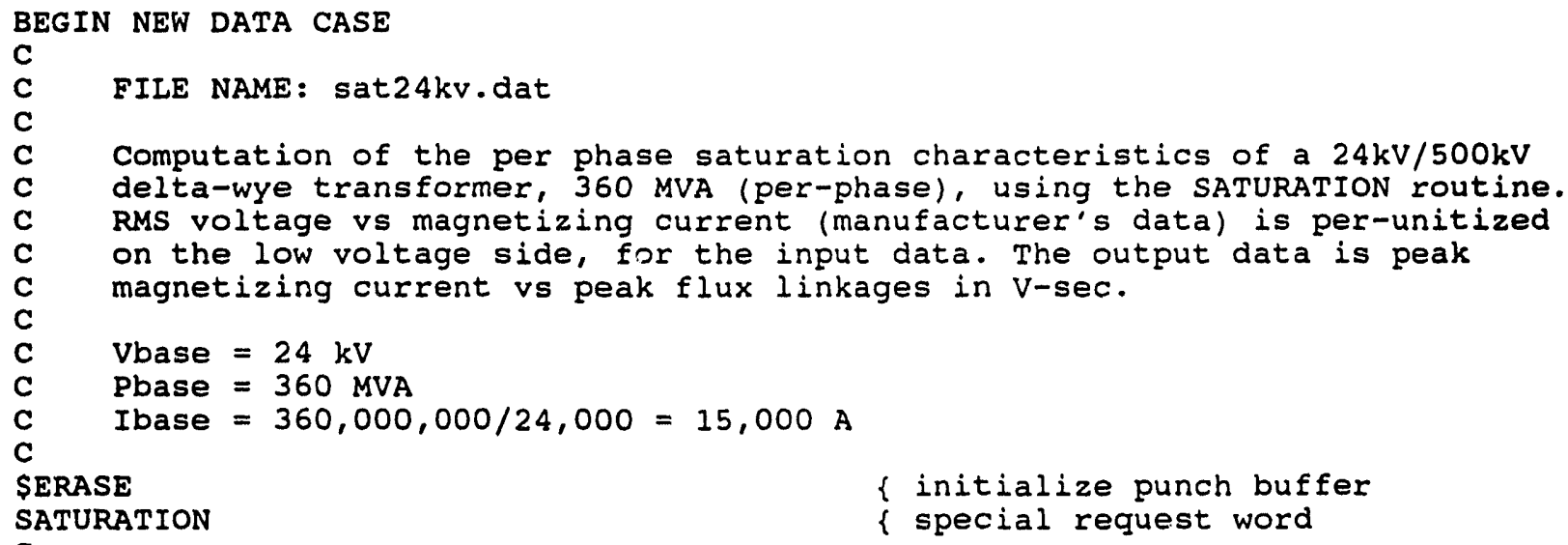

C

C

C

Magnetizing test data

$$
\begin{array}{r}
\text { Irms } \\
5.696 \mathrm{E}-3 \\
0.02092
\end{array}
$$$$
\begin{aligned}
& \text { Vrms } \\
& 1.0 \\
& 1.1
\end{aligned}
$$

C

C

Data terminator

\$PUNCH, sat $24 \mathrm{kV}$. inc

BLANK END OF DATA FILE

$\{$ flush punch buffer into file sat $24 \mathrm{kv}$.inc

BLANK END OF ALL CASES

\section{Saturation Output Data:}

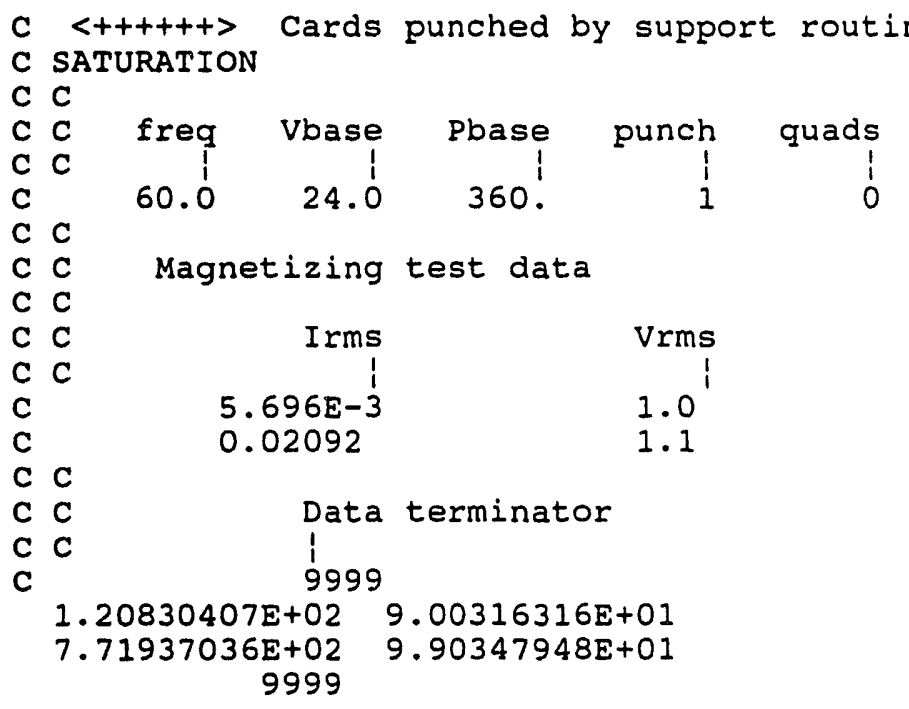




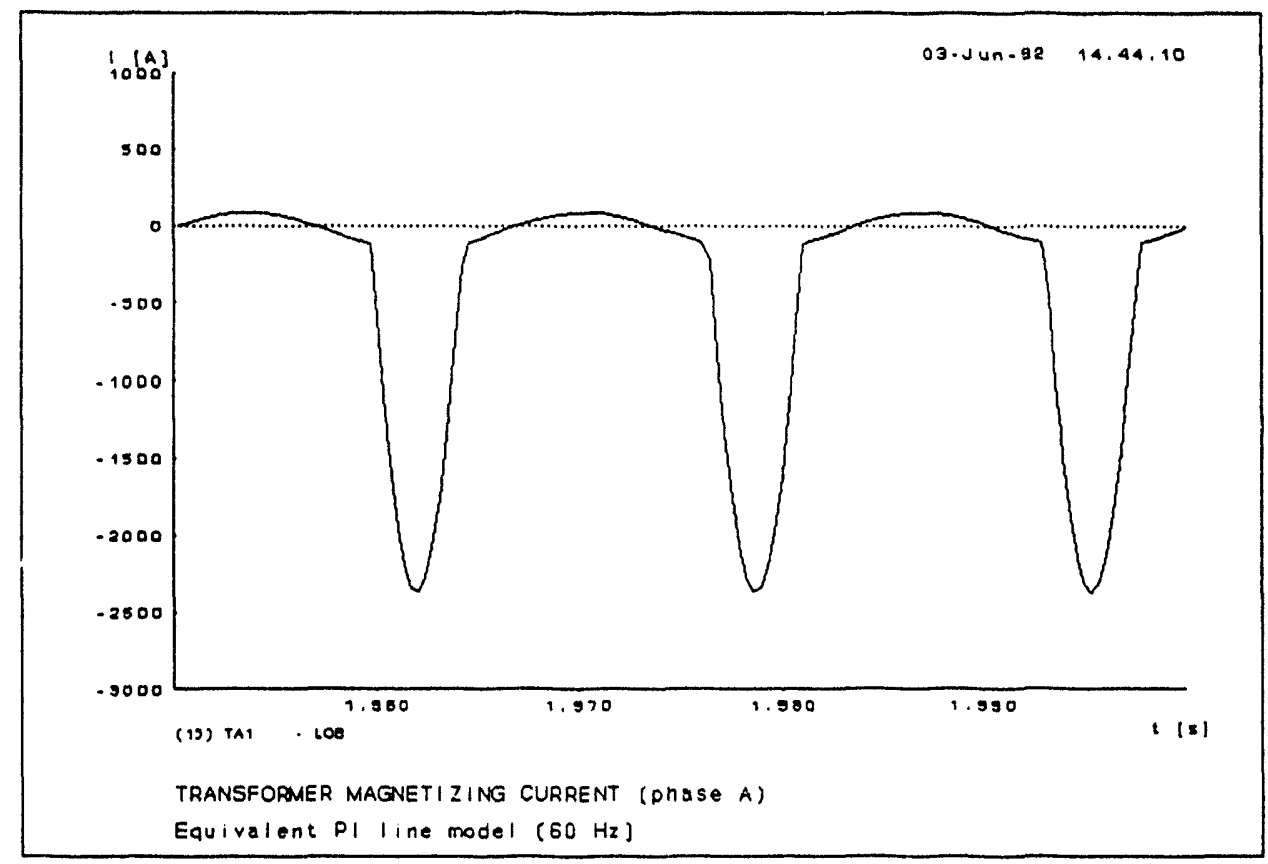

Fig. D-1. Transformer magnetizing current (phase A), $60 \mathrm{~Hz}$ equivalent PI line model.

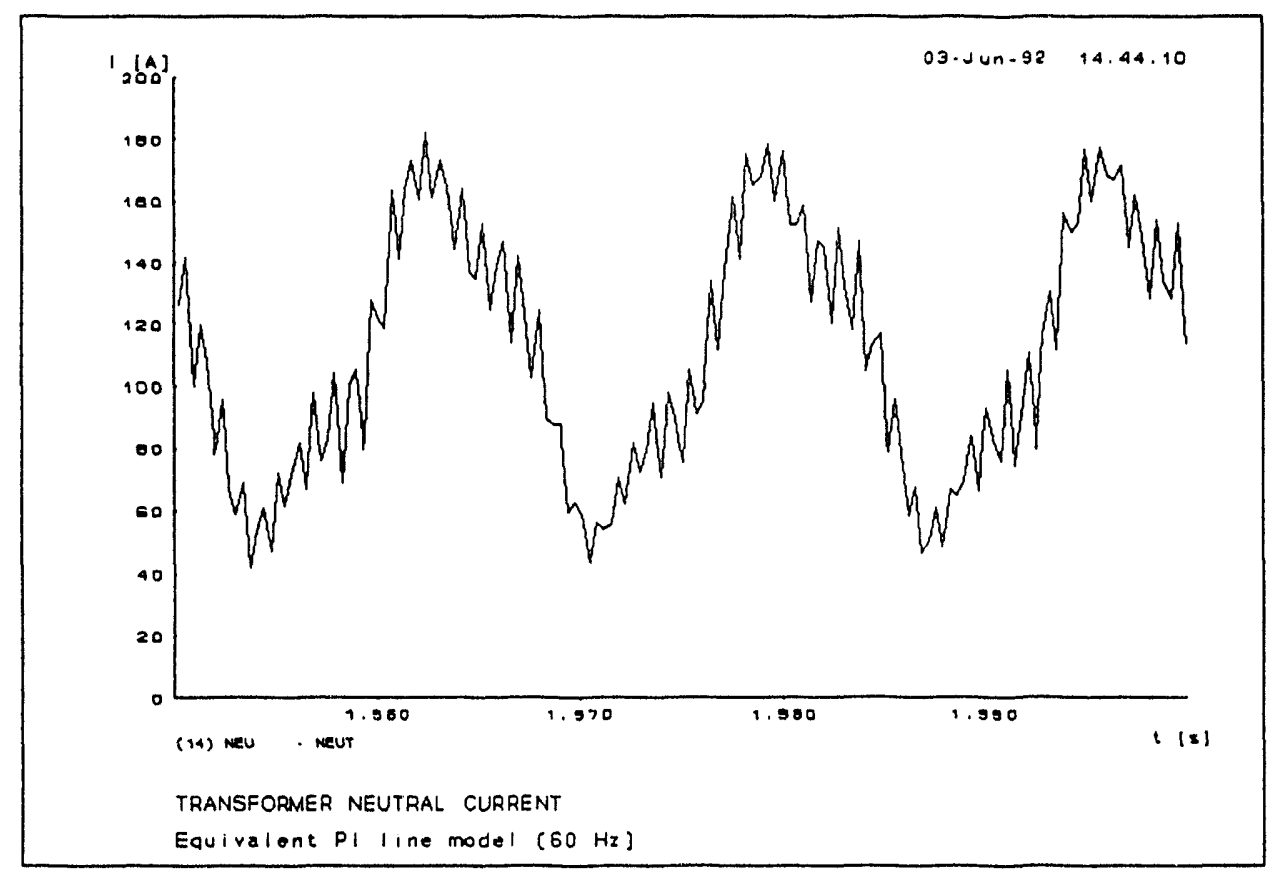

Fig. D-2. Transformer neutral current, $60 \mathrm{~Hz}$ equivalent PI line model.

$$
D-5
$$



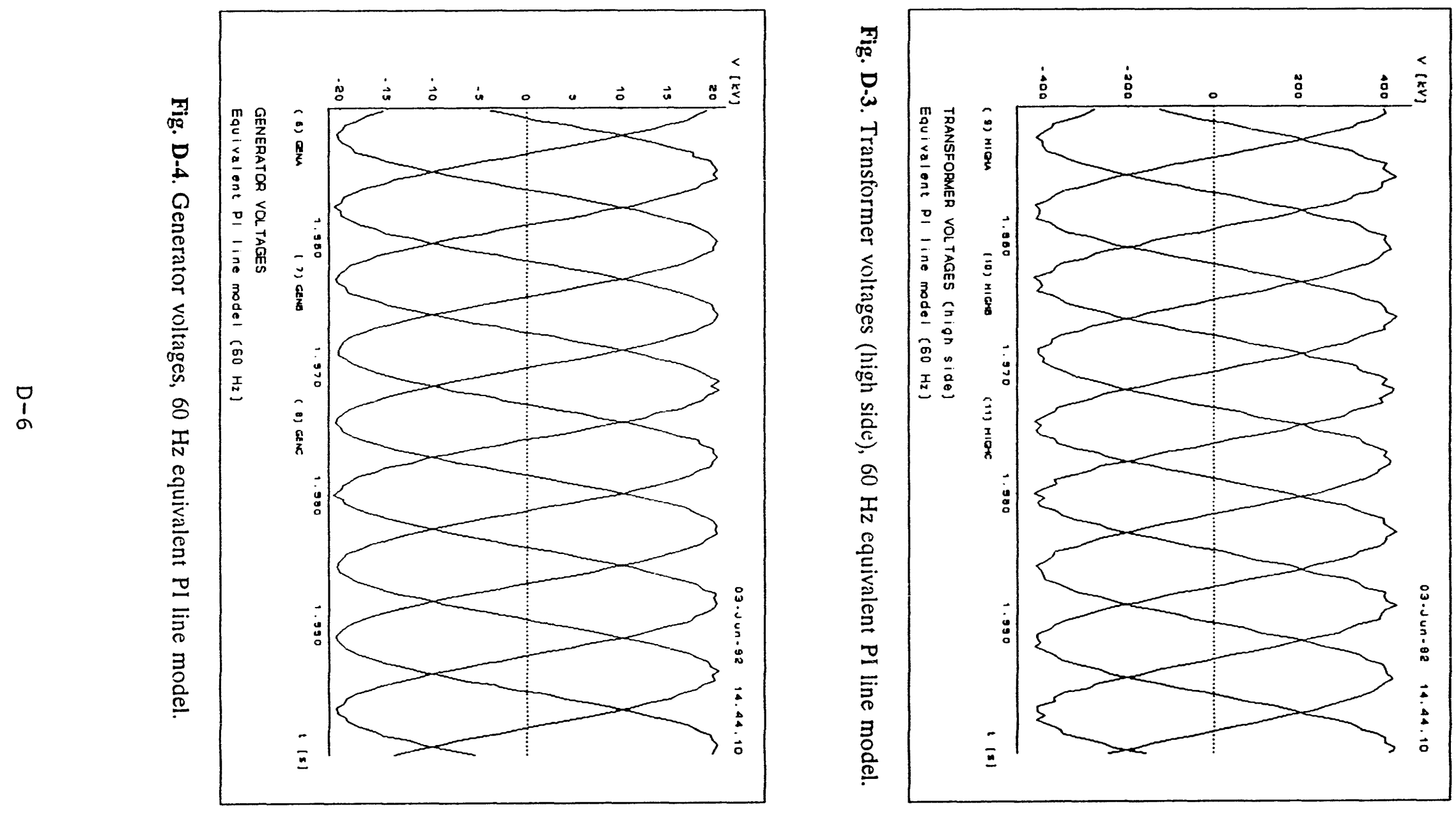


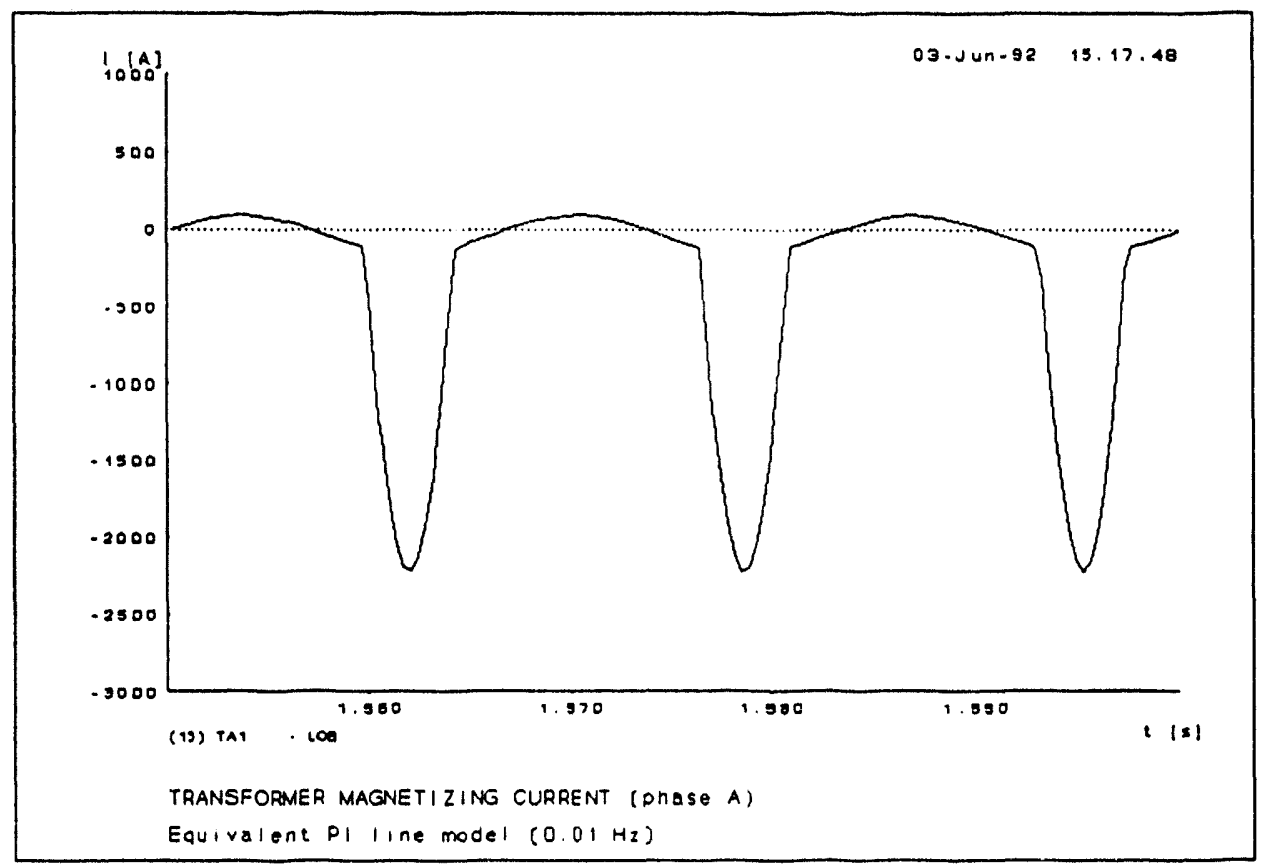

Fig. D-5. Transtormer magnetizing current (phase A), $0.01 \mathrm{~Hz}$ equivalent PI line model.

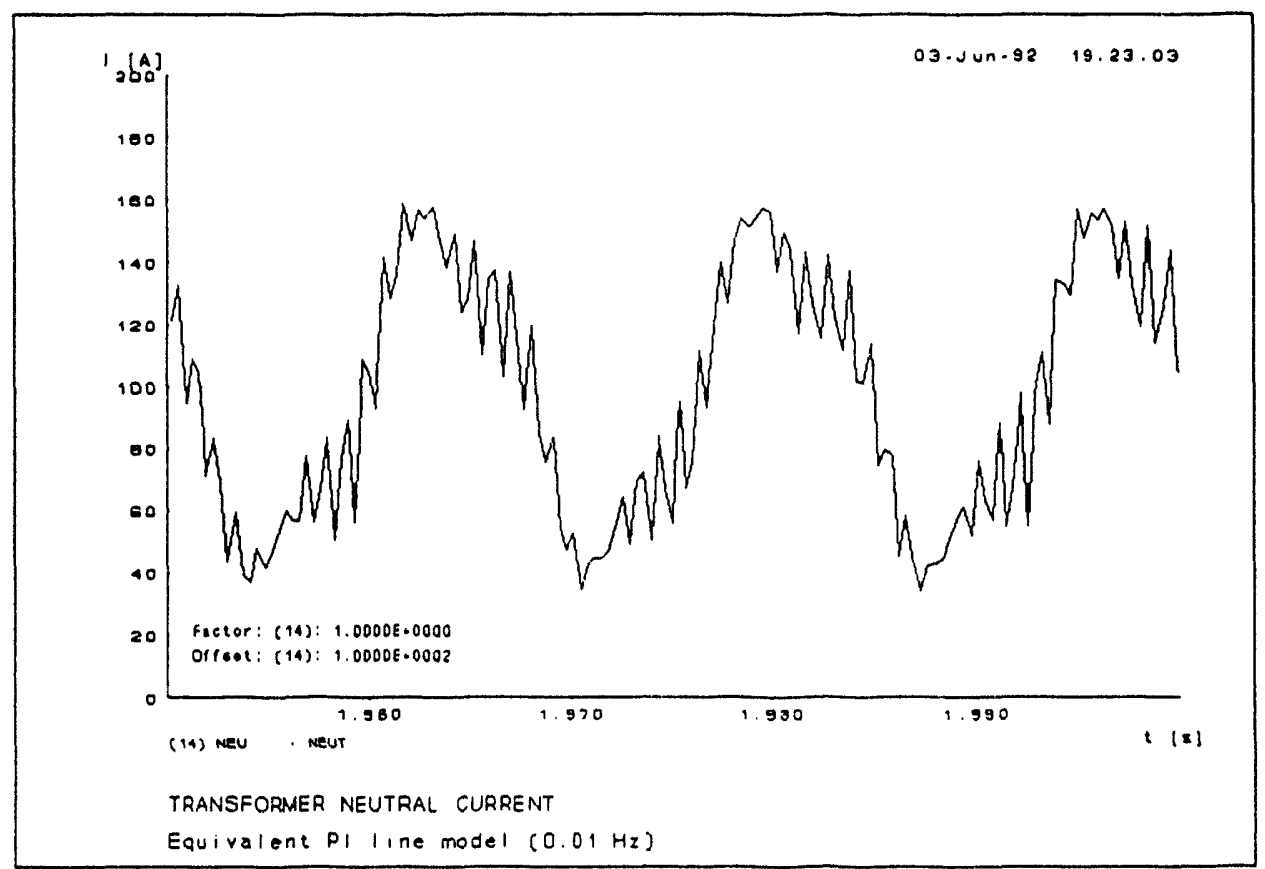

Fig. D.6. Transformer neutral current, $0.01 \mathrm{~Hz}$ equivalent PI line model. 


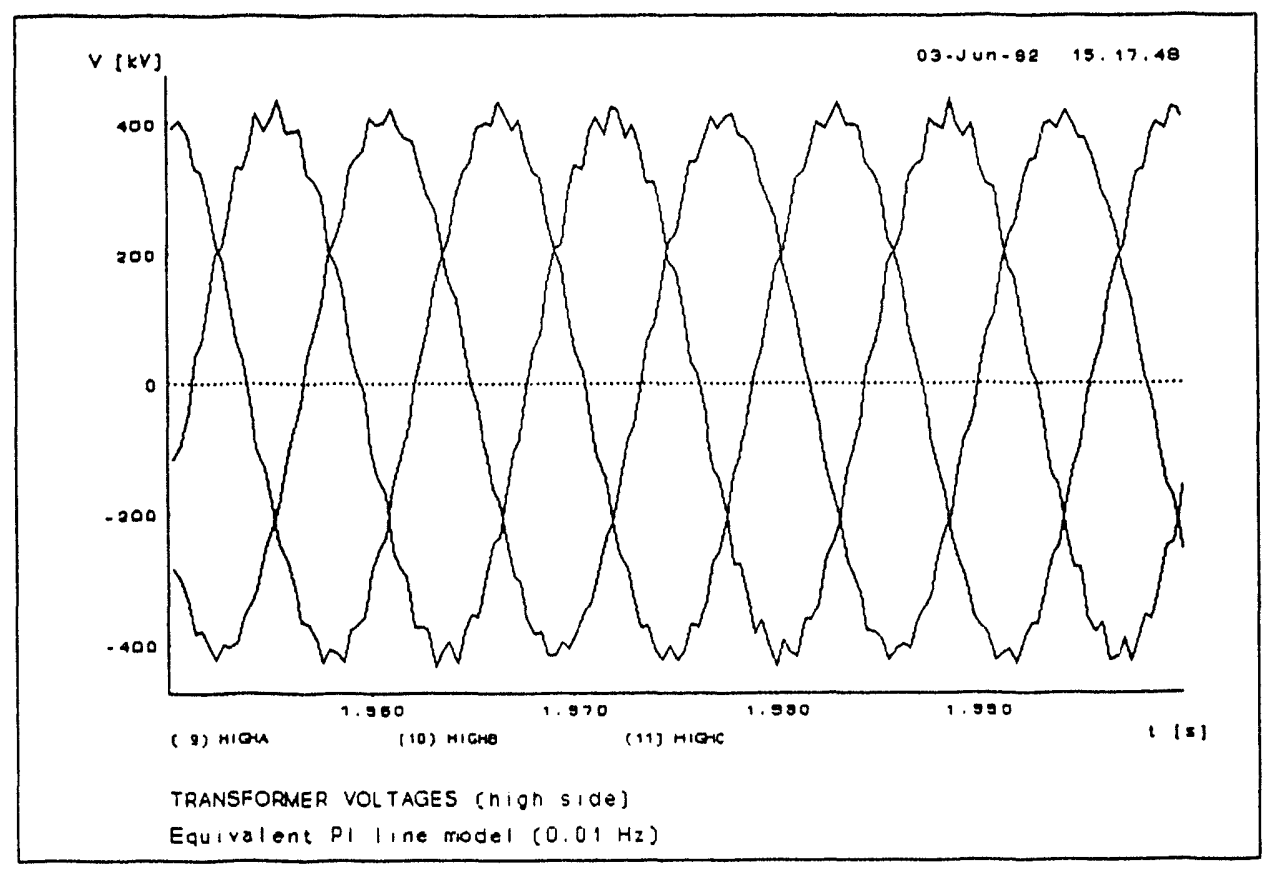

Fig. D-7. Transformer voltages (high side), $0.01 \mathrm{~Hz}$ equivalent PI line model.

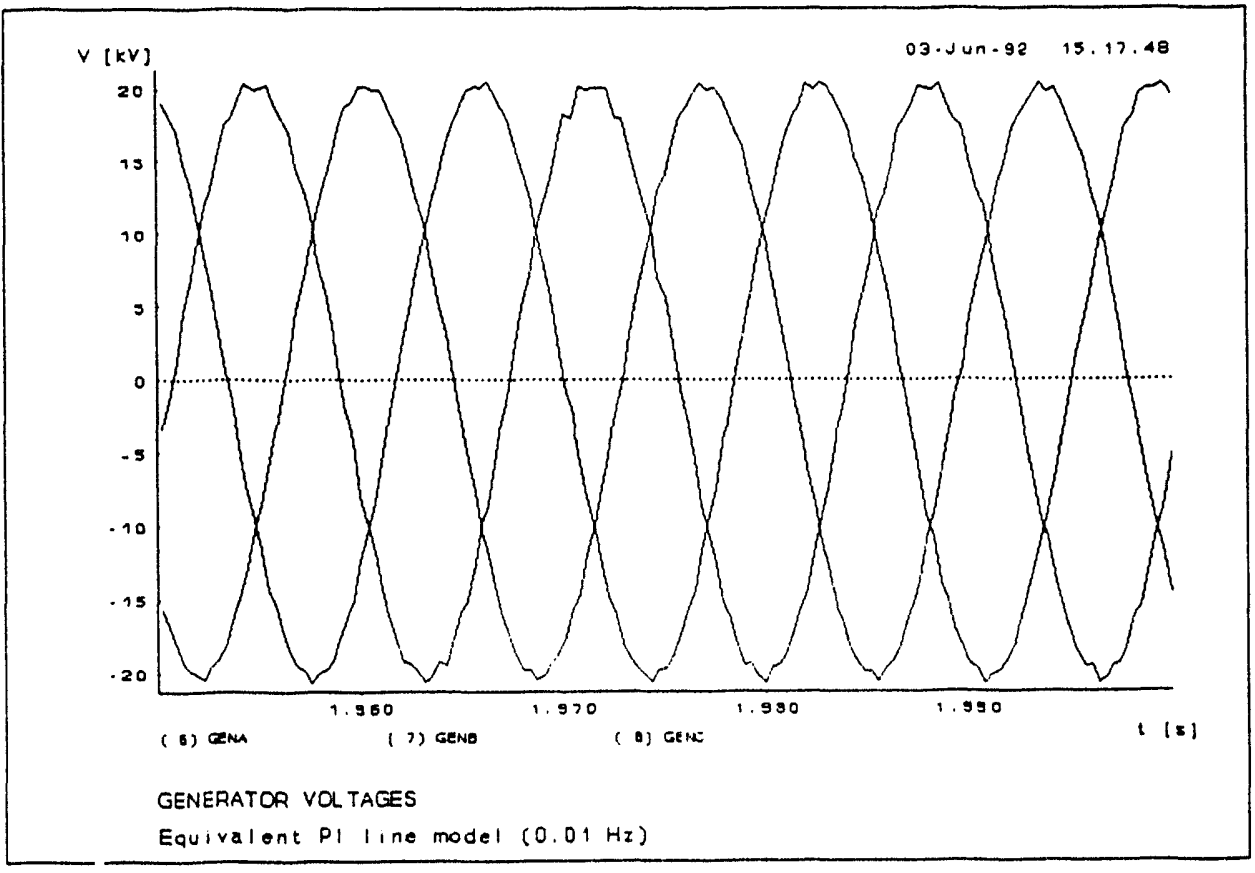

Fig. D-8. Generator Voltages, $0.01 \mathrm{~Hz}$ equivalent PI line model. 


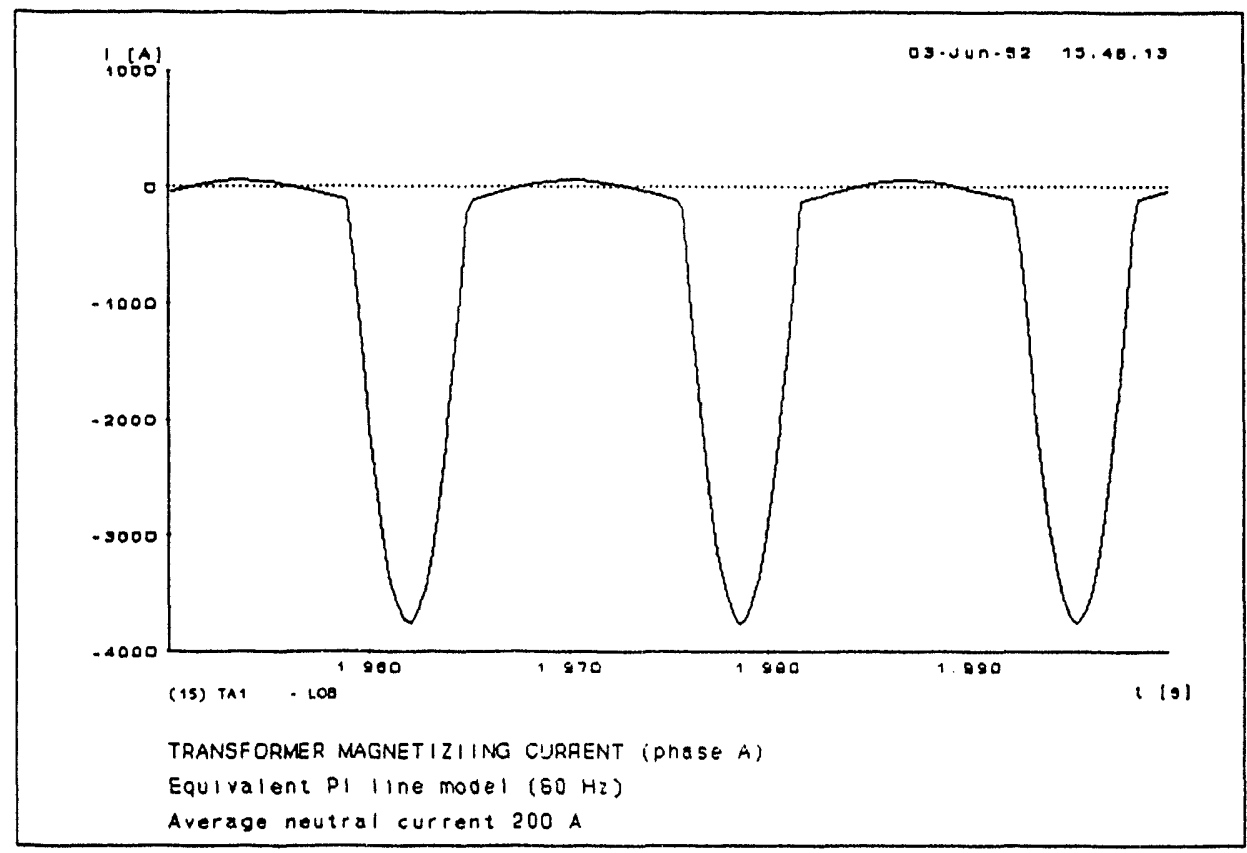

Fig. D-9. Transformer magnetizing current, with $200 \mathrm{~A}$ neutral current, $60 \mathrm{~Hz}$ equivalent PI line model.

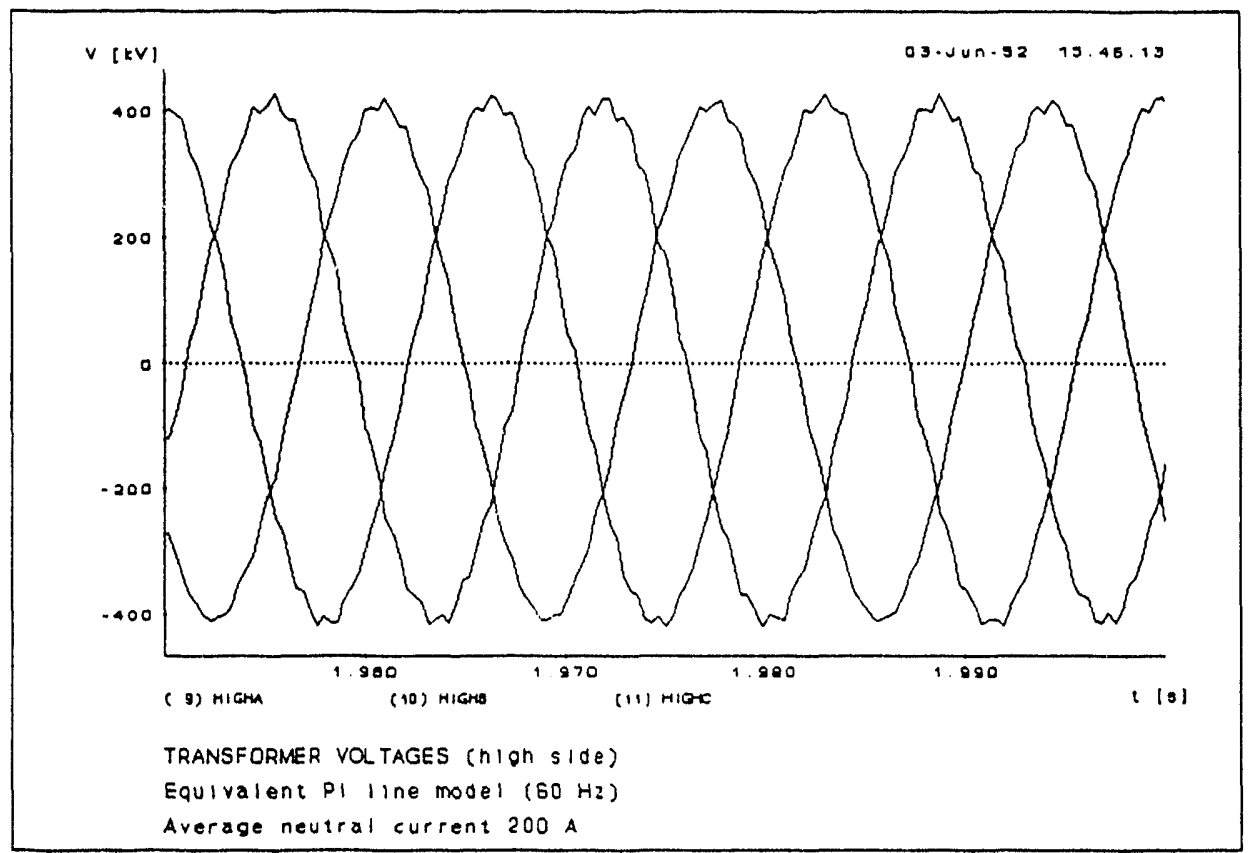

Fig. D-10. Transformer voltages (high side), with 200 A neutral current, $60 \mathrm{~Hz}$ equivalent PI line model. 


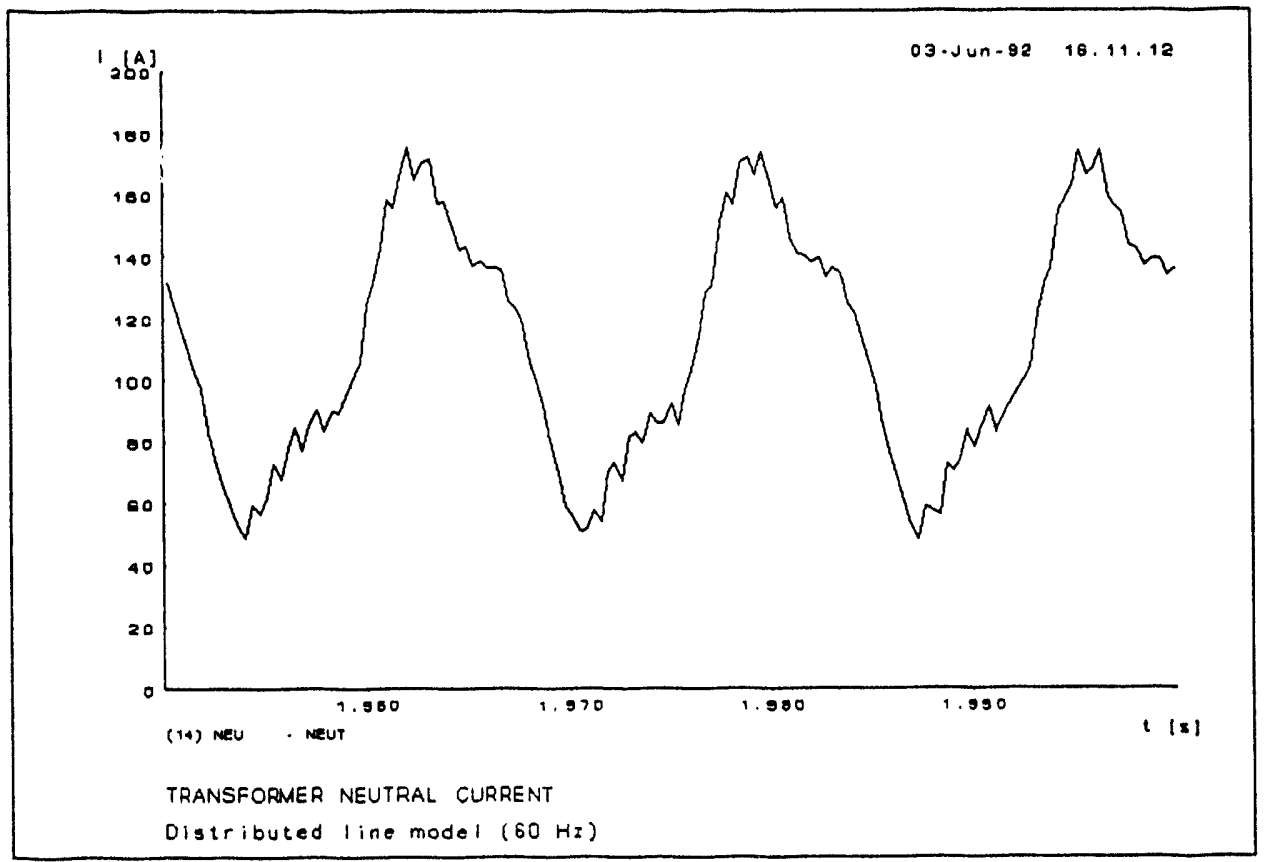

Fig. D-11. Transformer neutral current, $60 \mathrm{~Hz}$ distributed line model.

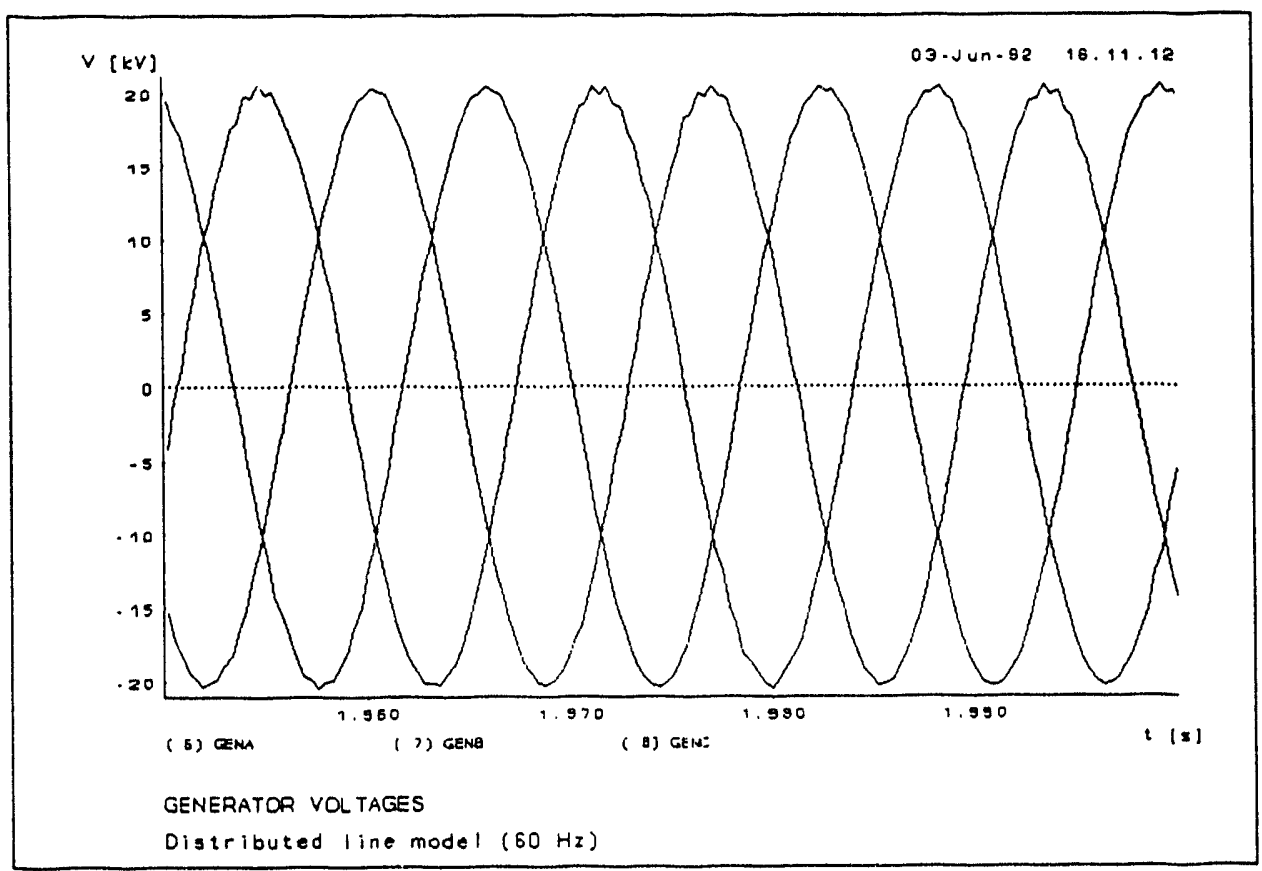

Fig. D-12. Generator voltages, $60 \mathrm{~Hz}$ distributed line model. 


\section{APPENDIX E:}

MODEL DATA AND INPUT FILES FOR EMTP SIMULATION

$345 \mathrm{kV}$ Overhead Transmission Line Data

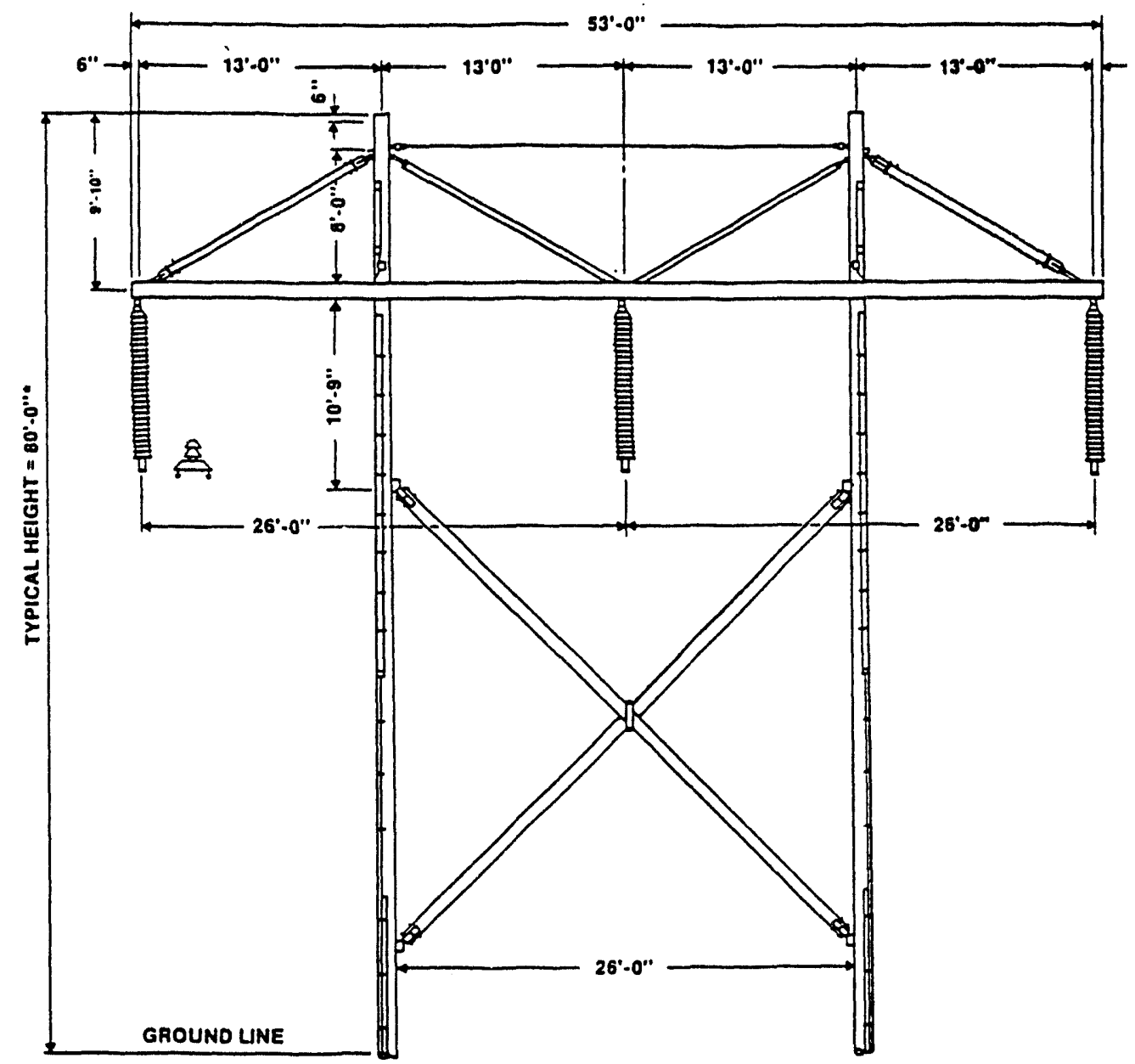

Fig. E-1. Typical $345 \mathrm{kV}$ transmission tower and conductor layout.

ACSR conductor data: Twin bundle per phase (18" scparation)

1.302" outside diameter (Bunting 45/7)

0.326 " core diameter

21 tt sag att midspan

$0.0787 \mathrm{ohm} / \mathrm{mi}$ de resistance

Shield wire data:

7/16" outside diameter (EHS steel)

10.7 ft sag at midspin

4.61 ohms/mi de resistance

$$
E-1
$$




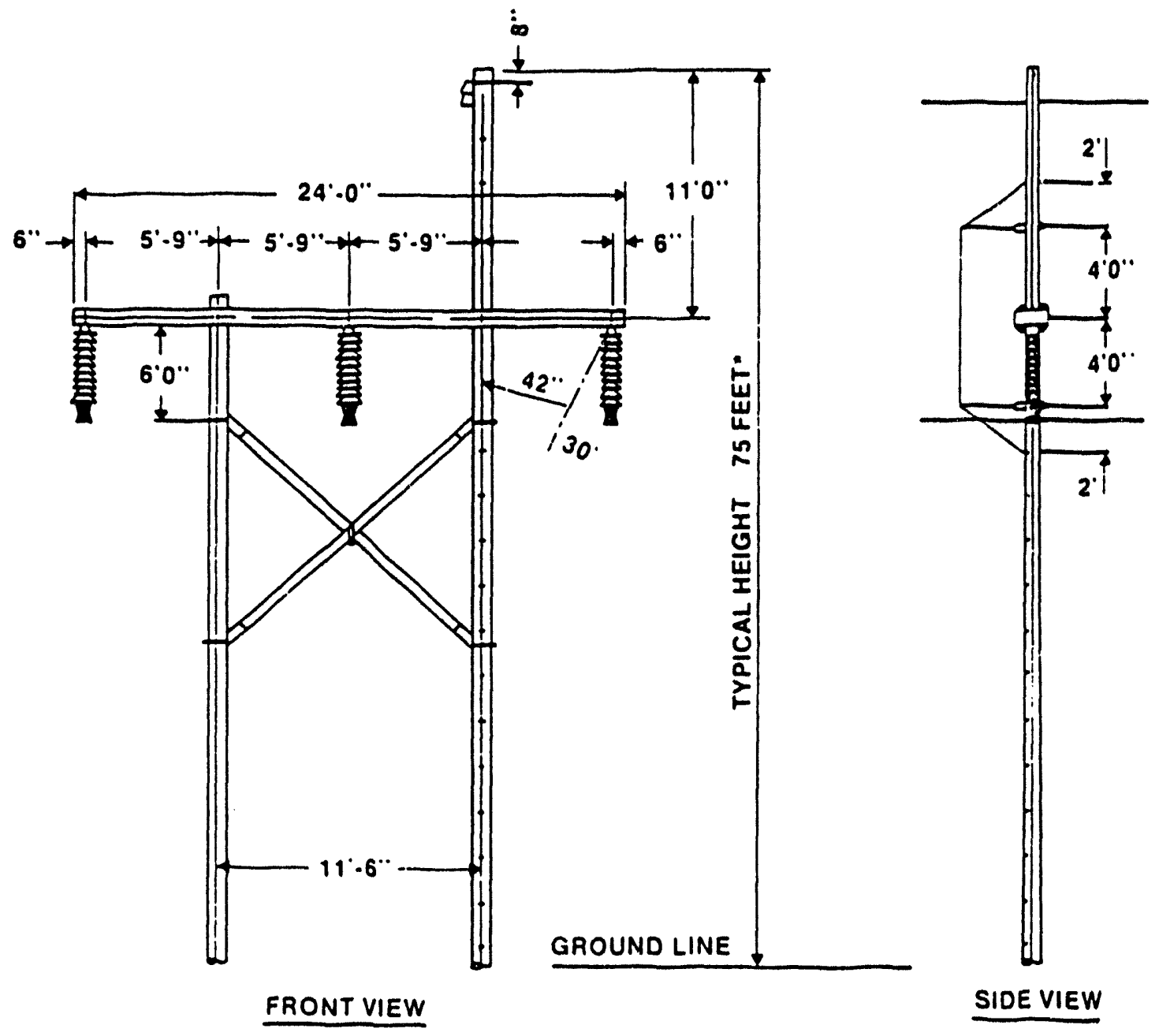

Fig. E-2. Typical $115 \mathrm{kV}$ transmission tower and conductor layout.

ACSR conductor data: $1.108^{\prime \prime}$ outside diameter

$0.408^{\prime \prime}$ core diamater

$16 \mathrm{ft}$ sag at midspan

$0.1180 \mathrm{ohm} / \mathrm{mi} \mathrm{dc}$ resistance

Shield wire data:

$3 / 8^{\text {" }}$ outside diameter (HS steel)

$8 \mathrm{ft}$ sag at midspan

$6.51 \mathrm{ohms}$ imi dc resistance 
SATURATION CHARACTERISITICS

Generator Step-up Transformer

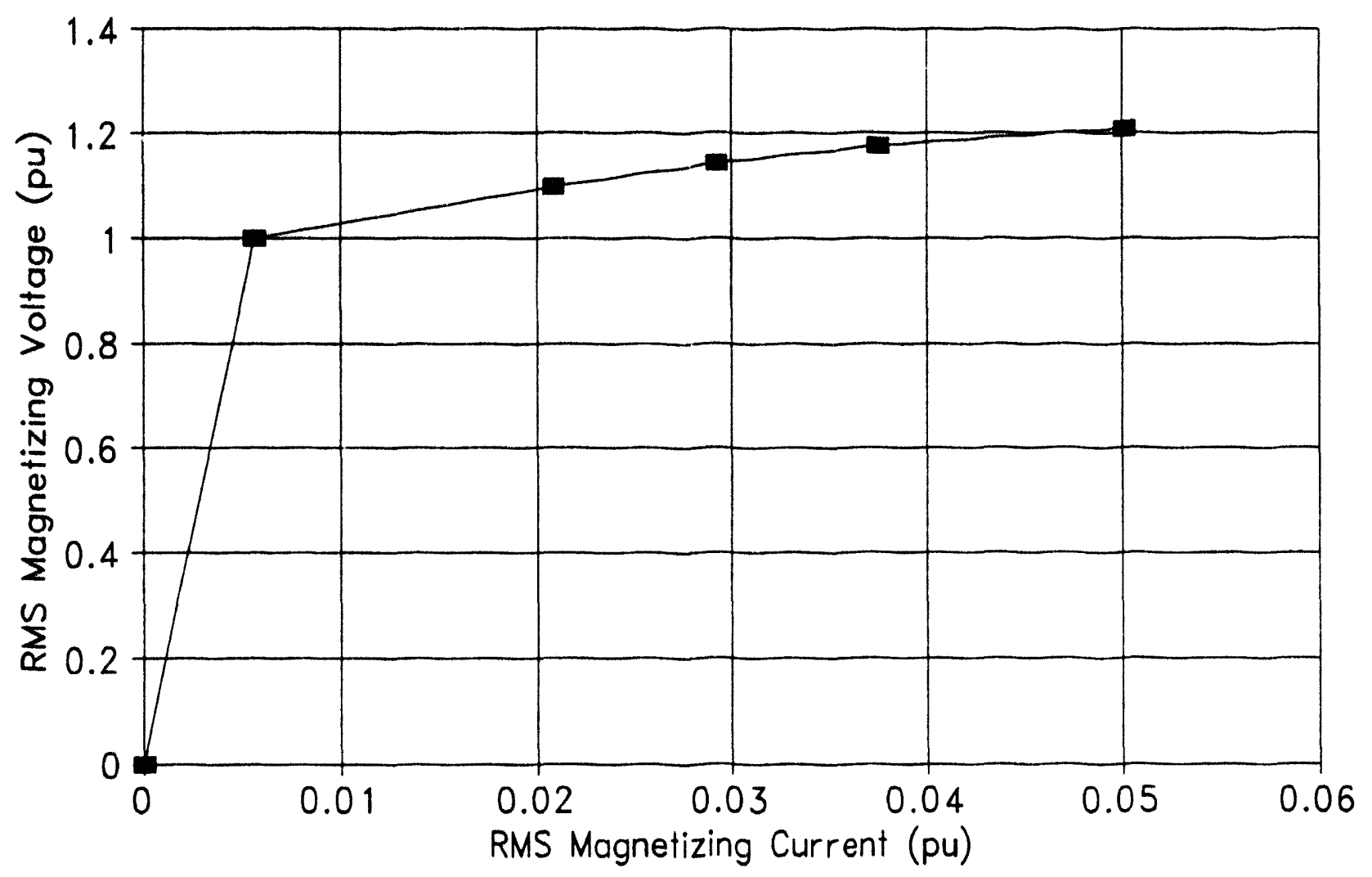

Fig. E-3. Saturation characteristics of generator step-up transformer.

Transformer rating: 408/457 MVA per phase (OA/FA), 345/23.85 kV

Base impedances: $1.394 \Omega$ (low side), $97.24 \Omega$ (high side)

Per phase impedance: $16 \%$ total, $9.77(10)^{-4}$ pu resistance per winding

Core loss per phase: $264.9 \mathrm{~kW}$

Model parameters: $\quad R_{1}=0.00136 \Omega, X_{1}=0.1115 \Omega$ (LV winding)

$R_{2}=0.095 \Omega, X_{2}=7.7792 \Omega$ ( $\mathrm{HV}$ winding)

$R_{\text {mas }}=2148 \Omega$ (core losses)

$I_{\text {mag }}=137 \mathrm{~A}$ (peak magnetizing current at knee)

$\lambda_{\text {mag }}=89.467 \mathrm{~V}$-s (peak flux linkage at knee)

Magnetizing branch on LV winding 
SATURATION CHARACTERISITICS

Normal Station Service Transformer

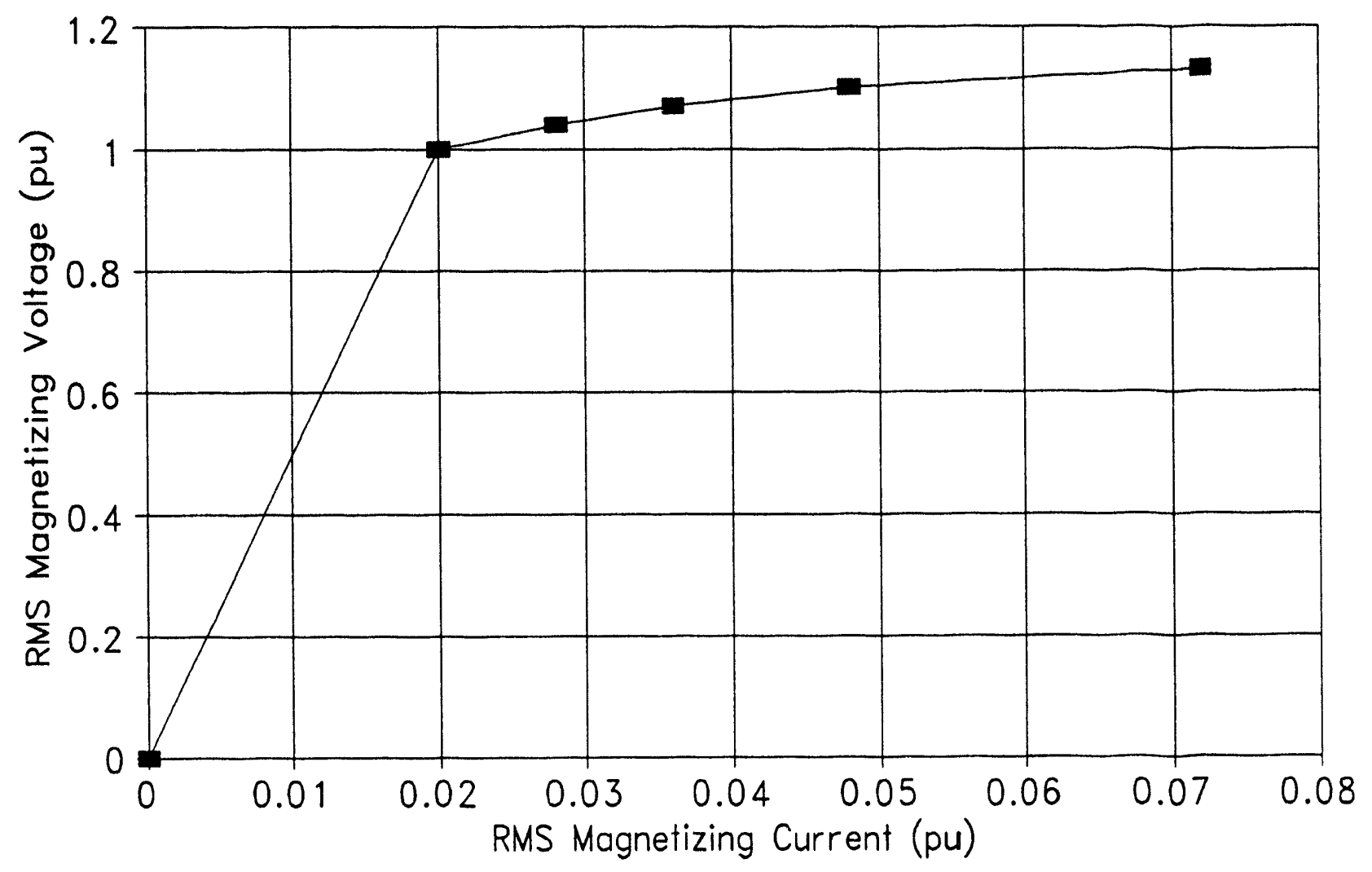

Fig. E-4. Saturation characteristics for normal station service transformer.

Transformer rating: $100 \mathrm{MVA}$ (FOA), $24.9 / 13.8 \mathrm{kV}$

Base impedances: $1.9044 \Omega$ (low side), $18.6 \Omega$ (high side)

Per phase impedance: $7.14 \%$ total, $1.5(10)^{-3}$ pu resistance per winding

Core loss (three-phase): $126.5 \mathrm{~kW}$

Model parameters: $\quad R_{1}=0.0279 \Omega, X_{1}=0.664 \Omega$ (HV winding)

$$
\begin{aligned}
& R_{2}=R_{3}=0.00286 \Omega, X_{2}=X_{3}=0.068 \Omega \text { (LV windings) } \\
& R_{\text {mag }}=14703 \Omega \text { (core losses) } \\
& I_{\text {mag }}=37.87 \mathrm{~A} \text { (peak magnetizing current at knee) } \\
& \lambda_{\text {mag }}=93.4 \mathrm{~V}-\mathrm{s} \text { (peak flux linkage at knee) } \\
& \text { Magnetizing branch on LV winding }
\end{aligned}
$$


Reserve Station Service Transformer (T3)

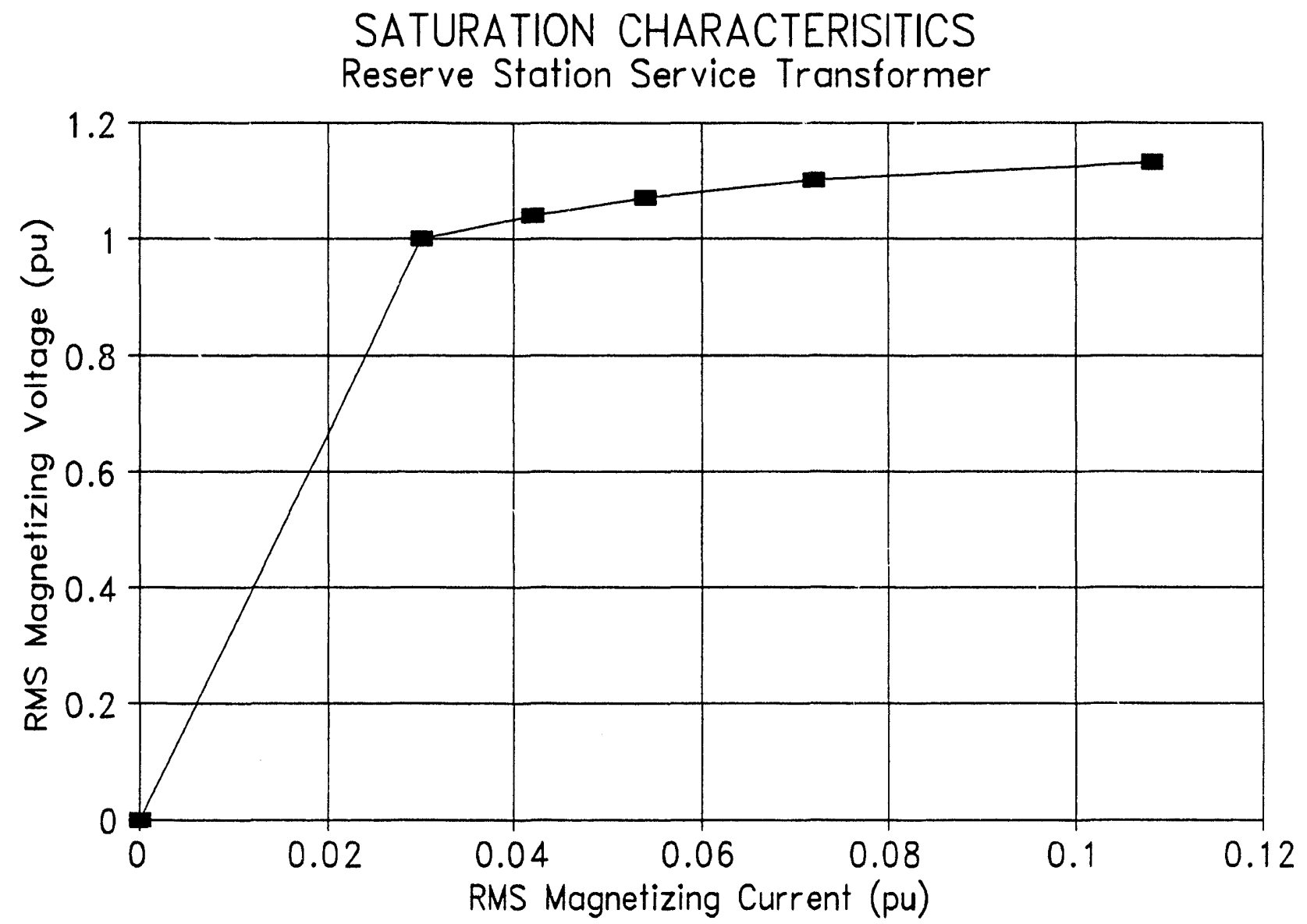

Fig. E-5. Saturation characteristics for reserve station service transformer.

Transformer rating: 42/56/70 MVA (0A/FA/FOA), 115/13.8/4.16 kV

Base inipedances: $314.88 \Omega(115 \mathrm{kV}), 4.534 \Omega(13.8 \mathrm{kV}), 1.236 \Omega(4.16 \mathrm{kV})$

Per phase impedance: $6.81 \%$ total, $1.85(10)^{-3}$ pu resistance per winding

Core loss (three-phase): $76.23 \mathrm{~kW}$

Model parameters: $\quad R_{1}=0.00229 \Omega, X_{1}=0.0421 \Omega$ (4.16 kV winding)

$$
\begin{aligned}
& R_{2}=0.5825 \Omega, X_{2}=10.72 \Omega(115 \mathrm{kV} \text { winding) } \\
& R_{3}=0.00839 \Omega, X_{3}=0.1544 \Omega(13.8 \mathrm{kV} \text { winding) } \\
& R_{\text {mag }}=681 \Omega \text { (core losses) } \\
& I_{\text {mag }}=142.79 \mathrm{~A} \text { (peak magnetizing current at knee) } \\
& \lambda_{\text {mag }}=15.605 \mathrm{~V}-\mathrm{s} \text { (peak flux linkage at knee) } \\
& \text { Magnetizing branch on } 4.16 \mathrm{kV} \text { winding }
\end{aligned}
$$

$$
\text { E-5 }
$$




\section{Auxiliary Boiler Transformer Data}

SATURATION CHARACTERISITICS

Auxiliary Boiler Transformer

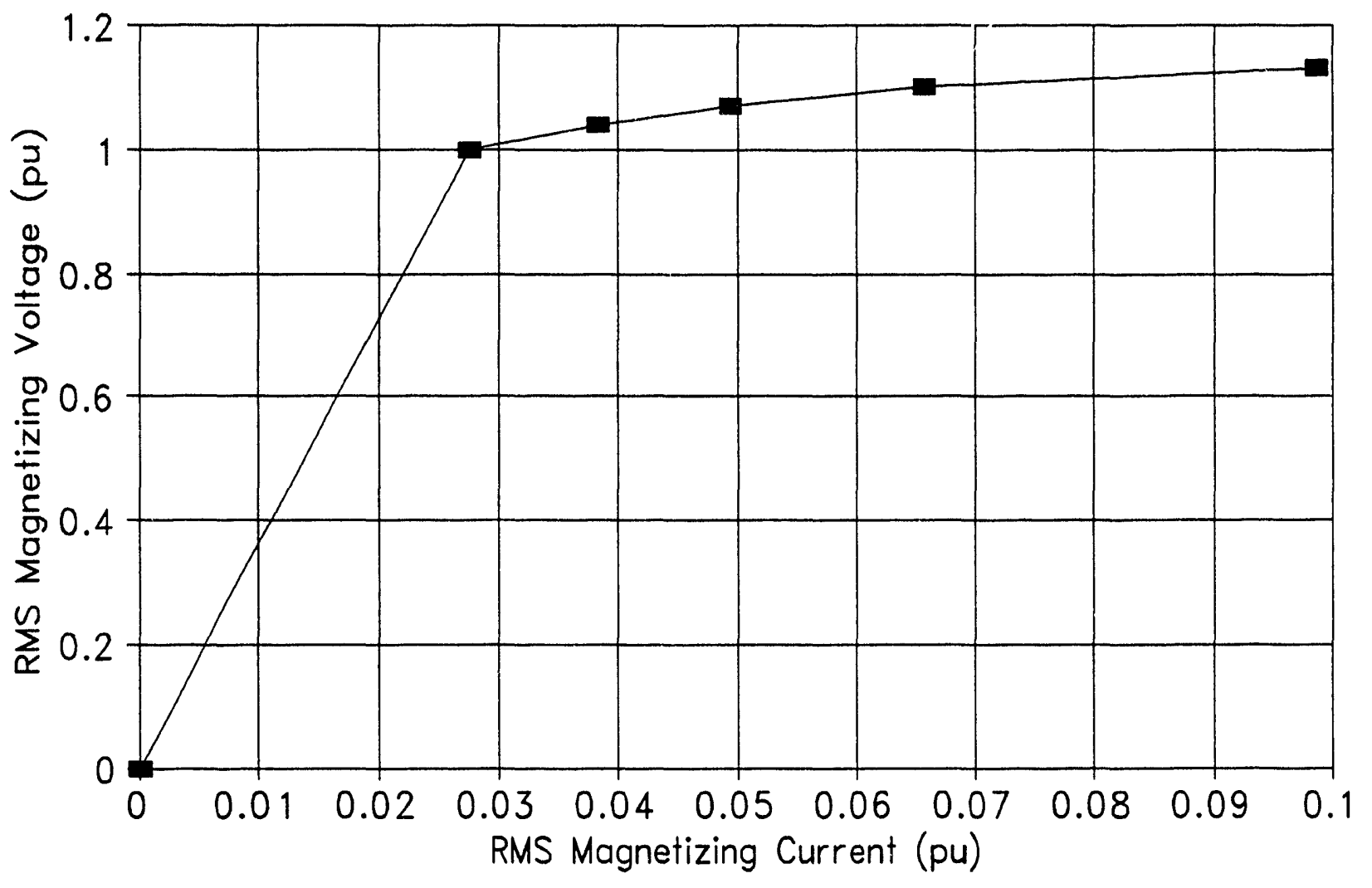

Fig. E-6. Saturation characteristics for auxiliary boiler transformer.

Transformer rating: 16.6/22.08/27.56 MVA (0A/FA/FOA), 115/13.8/4.16 kV

Base impedances: $796.7 \Omega(115 \mathrm{kV}), 11.47 \Omega(13.8 \mathrm{kV}), 3.1275 \Omega(4.16 \mathrm{kV})$

Per phase impedance: $6.9 \%$ total, $2.5(10)^{-3}$ pu resistance per winding

Core loss (three-phase): $40.26 \mathrm{~kW}$

Model parameters: $\quad R_{1}=0.00782 \Omega, X_{1}=0.1079 \Omega(4.16 \mathrm{kV}$ winding)

$R_{2}=1.9918 \Omega, X_{2}=27.5 \Omega$ (115 kV winding)

$\mathrm{R}_{3}=0.02868 \Omega, \mathrm{X}_{3}=0.3957 \Omega$ (13.8 $\mathrm{kV}$ winding)

$R_{\text {mag }}=1289.5 \Omega$ (core losses)

$I_{\text {mag }}=51.54 \mathrm{~A}$ (peak magnetizing current at knee)

$\lambda_{\text {mag }}=15.605 \mathrm{~V}$-s (peak flux linkage at knee)

Magnetizing branch on $4.16 \mathrm{kV}$ winding

$$
\text { E-6 }
$$




\section{Nonsafety-Related Transformer T4}

Transformer rating: 8.5/10.62 MVA (OA/FA), 13.2/4.16 kV

Base impedances: $2.036 \Omega$ (low side), 61.50 (high side)

Per phase impedance: $5.5 \%$ total, $2.6(10)^{-3}$ pu resistance per winding

Model parameters: $\quad R_{1}=0.1599 \Omega, X_{1}=1.691 \Omega(13.8 \mathrm{kV}$ winding)

$$
R_{2}=0.00529 \Omega, X_{2}=0.056 \Omega \text { (4.16 kV winding) }
$$

\section{Safety-Related Transformer T5}

Transformer rating: 1500/2025 kVA (OA/FA), 4160/600 V

Base impedances: $0.24 \Omega$ (low side), $34.6 \Omega$ (high side)

Per phase impedance: $4.8 \%$ total, $2.8(10)^{-3}$ pu resistance per winding

Model parameters: $\quad R_{1}=0.0969 \Omega, X_{1}=0.8304 \Omega(4160 \mathrm{~V}$ winding)

$$
R_{2}=0.00067 \Omega, X_{2}=0.00576 \Omega(600 \mathrm{~V} \text { winding) }
$$

\section{Nonsafety-Related Transformer T6}

Transformer rating: 1000/1350 kVA (OA/FA), 4160/600 V

Base impedances: $0.36 \Omega$ (low side), $51.92 \Omega$ (high side)

Per phase impedance: $5.75 \%$ total, $2.8(10)^{-3}$ pu resistance per winding

Model parameters: $\quad R_{1}=0.1454 \Omega, X_{1}=1.493 \Omega$ (4160 V winding)

$$
R_{2}=0.00101 \Omega, X_{2}=0.01035 \Omega(600 \mathrm{~V} \text { winding) }
$$

\section{Nonsafety-Related Transformer T7}

Transformer rating: $15 \mathrm{kVA}, 346.4 / 120 / 240 \mathrm{~V}$, single-phase

Impedance: $2.3 \%$ total, $7.9(10)^{-3}$ pu resistance per winding

Model parameters: $\quad \mathbf{R}_{1}=0.06326 \Omega, \mathrm{X}_{1}=0.0668 \Omega$ (346.4 $\mathrm{V}$ winding)

$$
R_{2}=0.00759 \Omega, X_{2}=0.008016 \Omega(120 \mathrm{~V} \text { winding })
$$

\section{Safety-Related Transformer T8}

Transformer rating: $25 \mathrm{kVA}, 346.4 / 120 / 240 \mathrm{~V}$, single-phase

Impedance: $2.5 \%$ total, $7.5(10)^{-3}$ pu resistance per winding

Model parameters: $\quad R_{1}=0.036 \Omega, X_{1}=0.048 \Omega$ (346.4 V winding)

$$
R_{2}=0.00432 \Omega, X_{2}=0.00576 \Omega(120 \mathrm{~V} \text { winding) }
$$




\section{Main Generator}

Generator rating: 1348.4 MVA, 0.9 PF lag, $25 \mathrm{kV}, 1800 \mathrm{rpm}, 60 \mathrm{~Hz}$

Subtransient reactance: $0.29 \mathrm{pu}=0.1344 \Omega$

\section{Feedwater Pump Motors}

Motor rating: 12,000 Hp, $13.8 \mathrm{kV}, 4$-pole IM, $1787 \mathrm{rpm}$

Stator resistance: 0.1210

Rotor resistance: $0.121 \Omega$ (referred to stator)

Stator leakage inductance: $0.004402 \mathrm{H}$

Rotor leakage inductance: $0.004402 \mathrm{H}$ (referred to stator)

Magnetizing inductance: $0.3241 \mathrm{H}$

Rotor and load inertia: $1349.9 \mathrm{~kg}-\mathrm{m}^{2}$ (137.54 N-m-sec $\left.{ }^{2}\right)$

Viscous damping constant: $4.888 \mathrm{~N}$-m-sec 


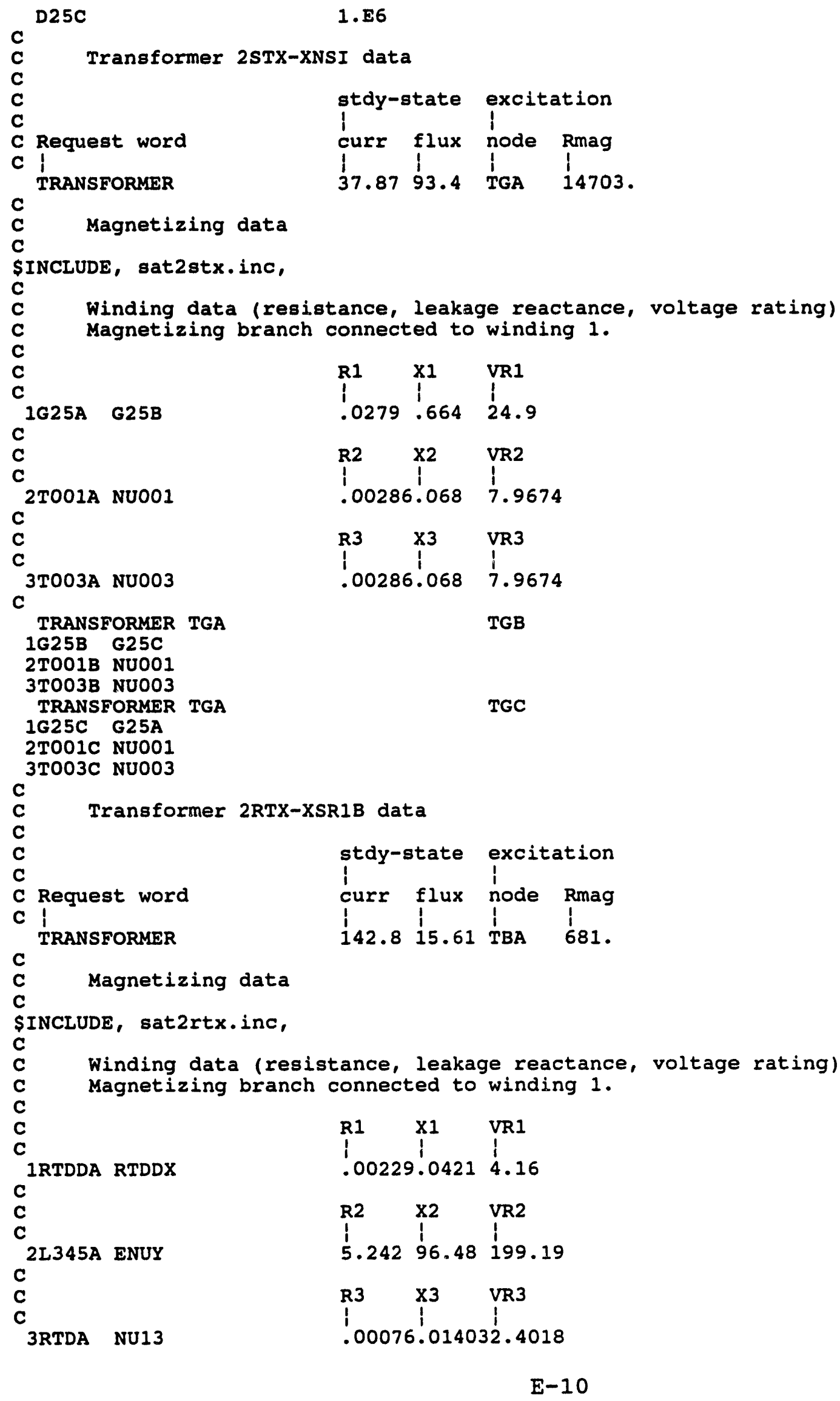

TGC

C Magnetizing data

\$INCLUDE, sat2rtx.inc,

C

C Winding data (resistance, leakage reactance, voltage rating)

C Magnetizing branch connected to winding 1 .

C

C

IRTDDA RTDDX

C

C

2L345A ENUY

C

C

3RTDA NU13 
C

TRANSFORMER TBA

TBB

1RTDDB RTDDC

2L345B ENUY

3RTDB NU13

TRANSFORMER TBA

TBC

1RTDDC RTDDA

2L345C ENUY

3RTDC NU13

C

NU13 1.28

ENU ENUY 1.E-6

C

C

C

C Request word

C I

TRANSFORMER

2ATX-XS3 data

C

C Magnetizing data termination flag.

stdy-state excitation

I

curr flux node Rmag TS3A

9999

C Winding data (resistance, leakage reactance, voltage rating)

C

C

C

1HI03A HI03B

R1 X1 VR1

C

C

C

2L003A NUS3

i.1599 1.691 is.2

TRANSFORMER TS3A

1HI03B HIO3C

2L003B NUS3

TRANSFORMER TS3A

1HI03C HIO3A

2L003C NUS3

C

c

Transformer $2 \mathrm{EJS}-\mathrm{X} 3 \mathrm{~A}$ data

C

C

C Request word

C I

TRANSFORMER

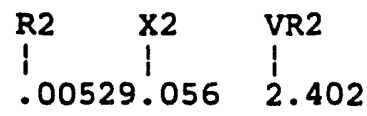

TS3B

TS3C

C

C Magnetizing data termination flag.

C

9999

C

C

C

C

1BUSDA BUSDB

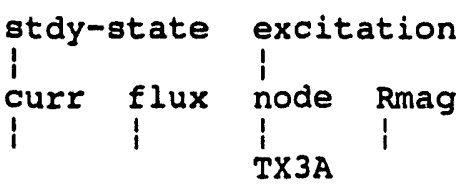

C

C

C

2T600A

Winding data (resistance, leakage reactance, voltage rating)

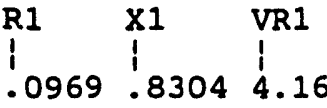

$\begin{array}{lll}R 2 & X 2 & V R 2\end{array}$

.00067 .00576 .3464

C 


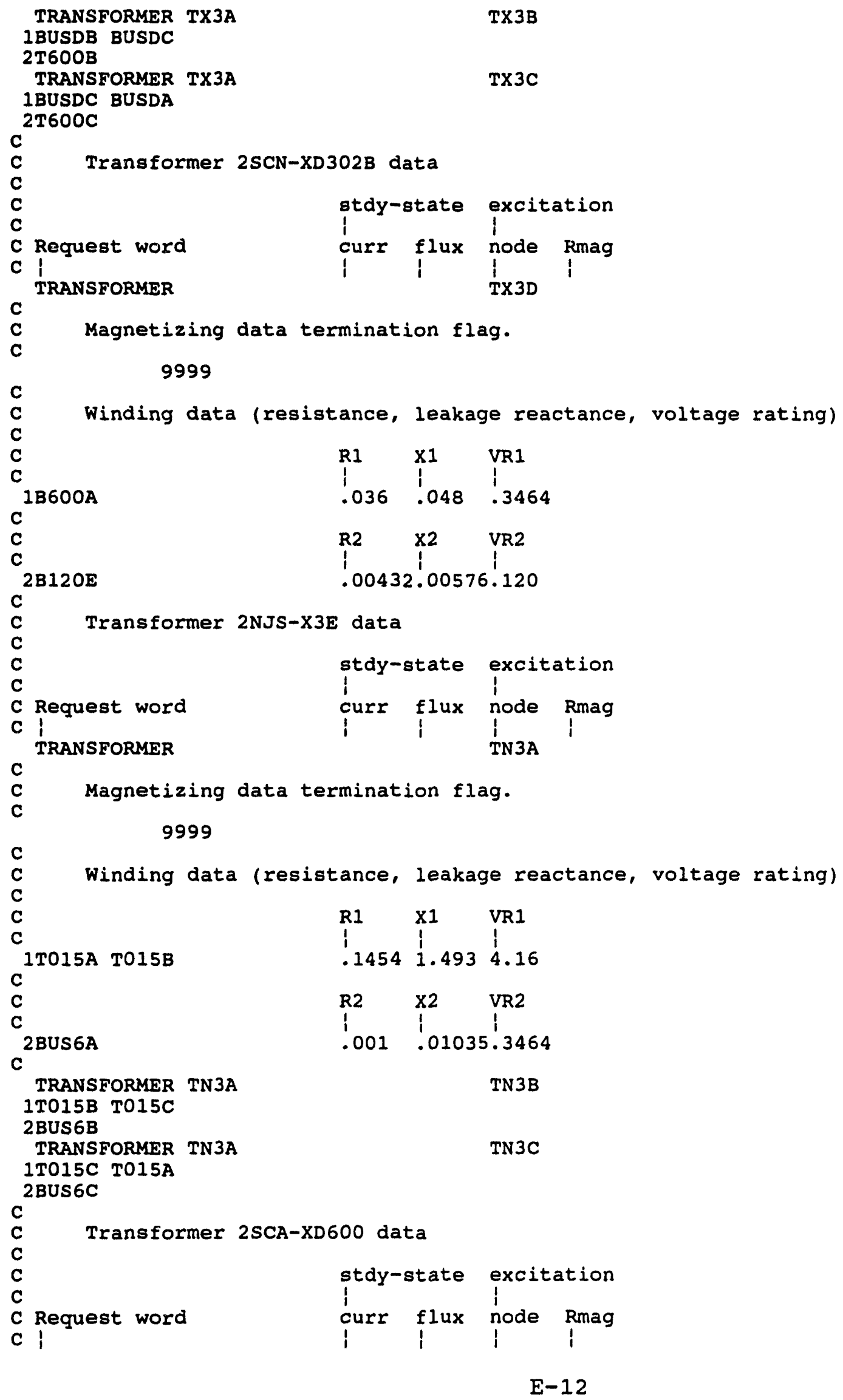

C

C Winding data (resistance, leakage reactance, voltage rating)

C

C

1T015A T015B

R1 X1 VR1

C

C

2BUS6A

$\begin{array}{ll}\text { I.145 I } & \text { I } \\ .14934 .16\end{array}$

R2 $\mathrm{X} 2 \quad$ VR2

i

$.001 \quad .01035 .3464$

TRANSFORMER TN3A TN3B 1T015B T015C 2BUS6B

TRANSFORMER TN3A TN3C

1T015C TO15A

2BUS6C

C Transformer 2SCA-XD600 data

C

C

C Request word

C I

excitation node Rmag

C 


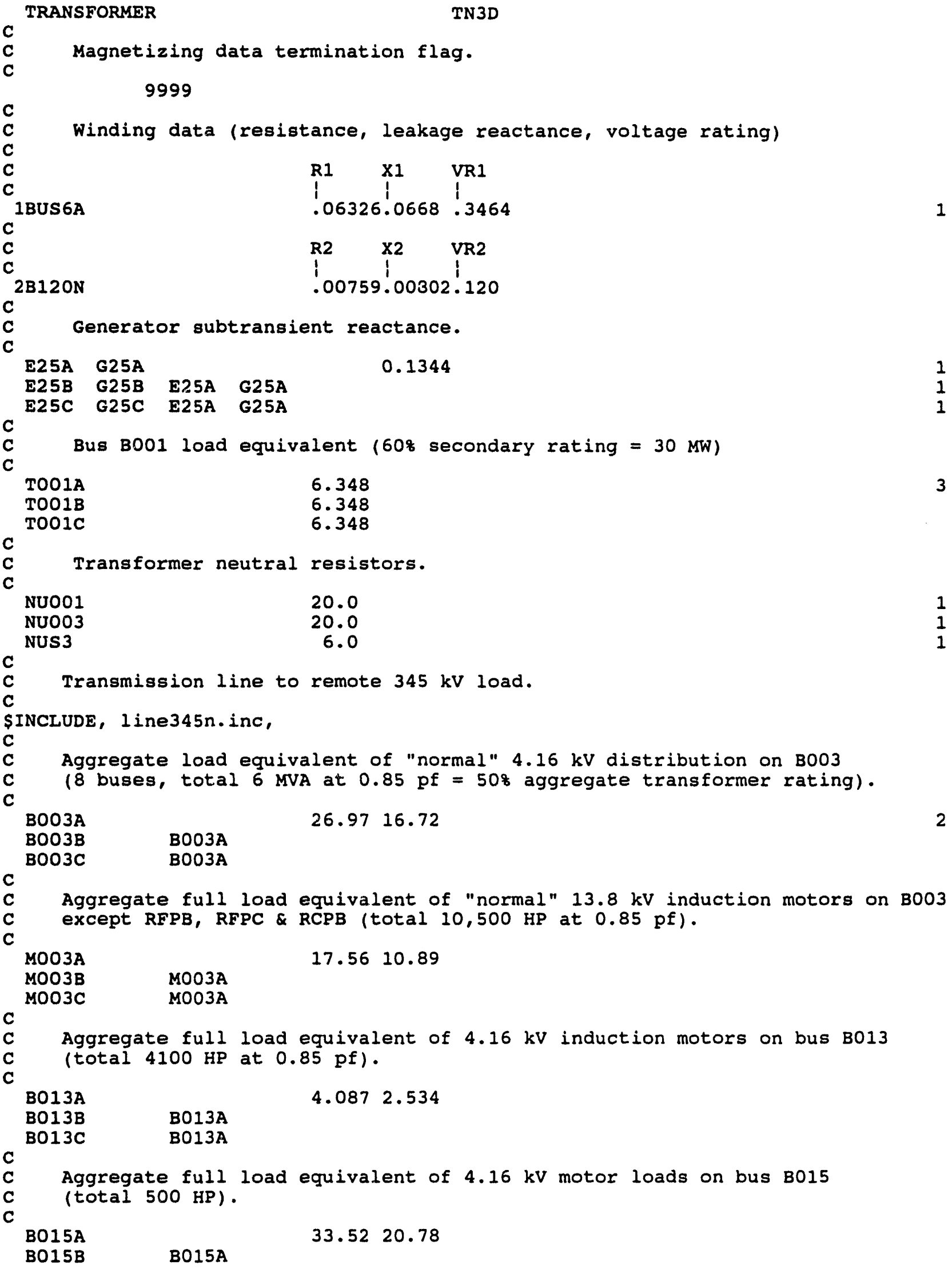




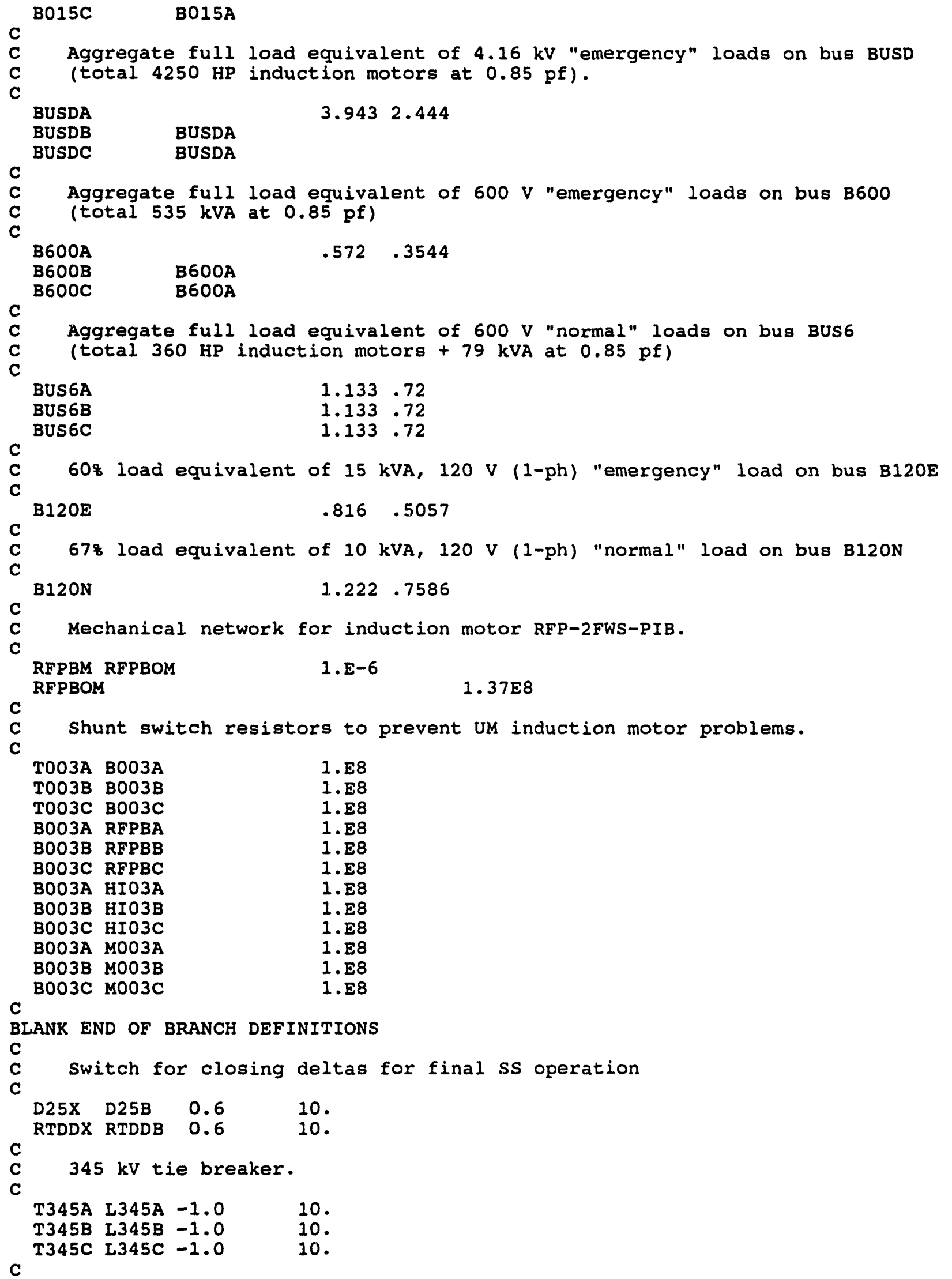




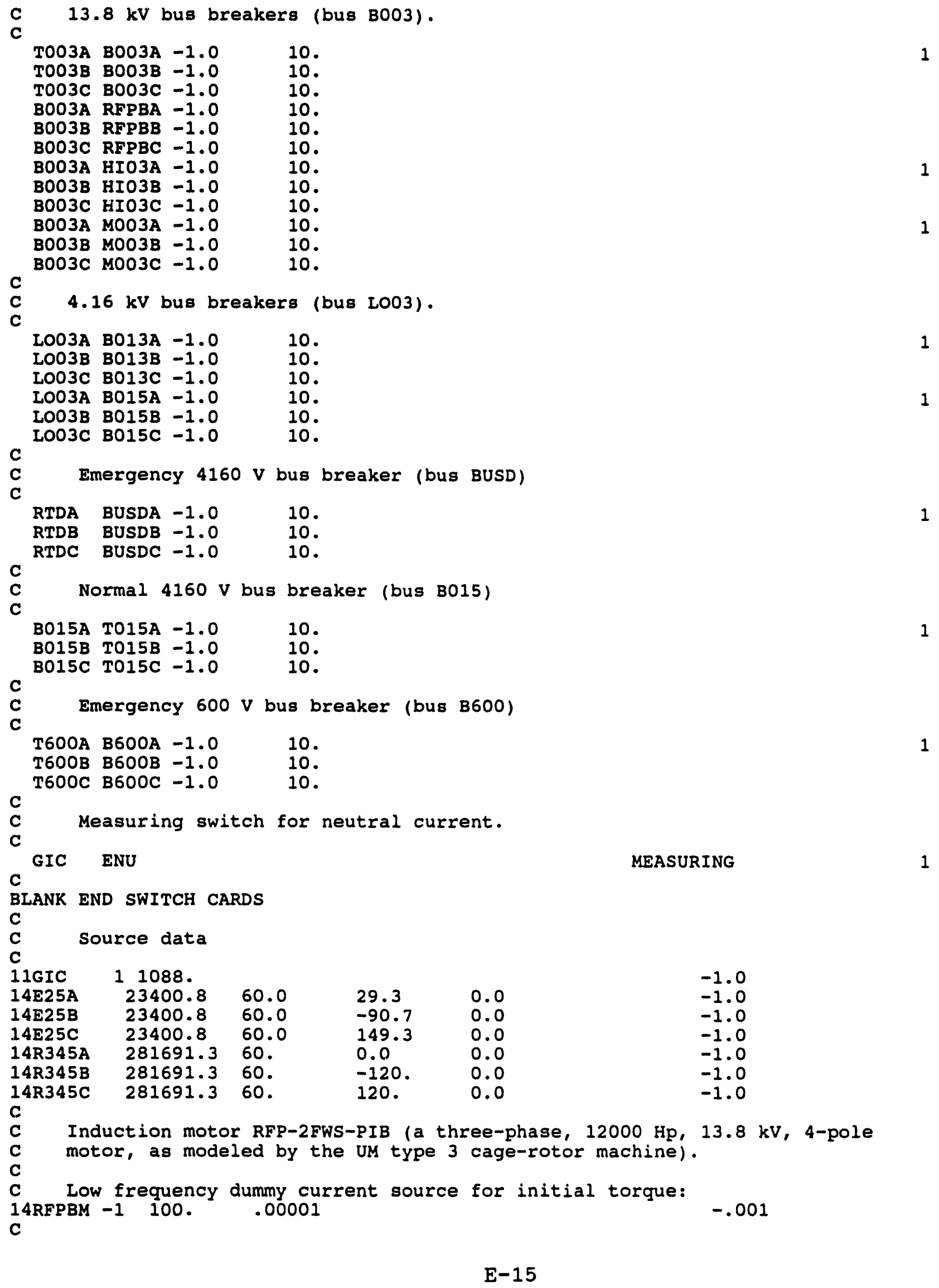


118t of U.M. data cards to represent 3-phase induction motor

01

(1) In column 2 means automatic initialization

BLANK card ending Clase-1 U.M. data

C

31 1111RFPBOM

2

0.18850

\{Type 3 cage-rotor

c

$\begin{array}{ccc}\text { C } & \text { Mutual inductance (Henry) } \\ 0.0 & 0.3241 & 0\end{array}$

0
0

C.

0.3241

.7222

RFPBM

10.72228 initial Elip

C stator windings (Ohm, Henry)

$0.121 \quad 0.004402 \quad$ RFPBA

$0.121 \quad 0.004402 \quad$ RFPBB

$0.121 \quad 0.004402 \quad$ RFPBC

10.0

10.0

10.0

C Rotor windings (Ohm, Henry)

$0.121 \quad 0.004402$

$0.121 \quad 0.004402$

10.0

C

BLANK card ending all U.M. data

C

BLANK END OF SOURCE DEFINITIONS

C

C Node voltages for plotting

C

G25A G25B G25C L345A L345B L345C LOO3A B60OA BUS6A B12OE B120N Y115A Y115B

Y115C RTDA RTDB RTDC

C

BLANK CARD ENDING PLOT CARDS

BLANK END OF SIMULATION

BEGIN NEW DATACASE

BLANK END OF ALL CASES 


\section{Line constants Routine:}

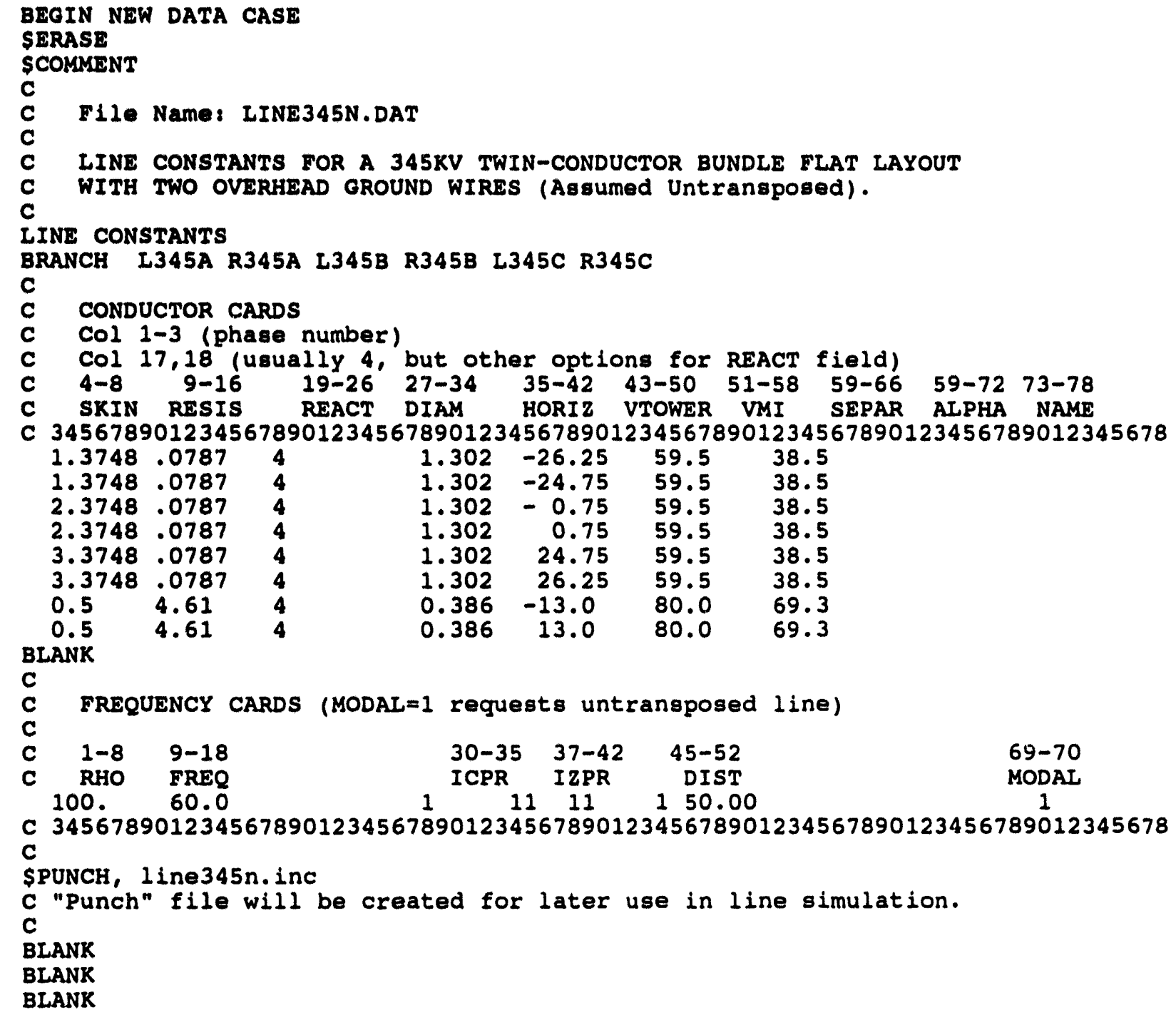

Distributed Line Model output Data:

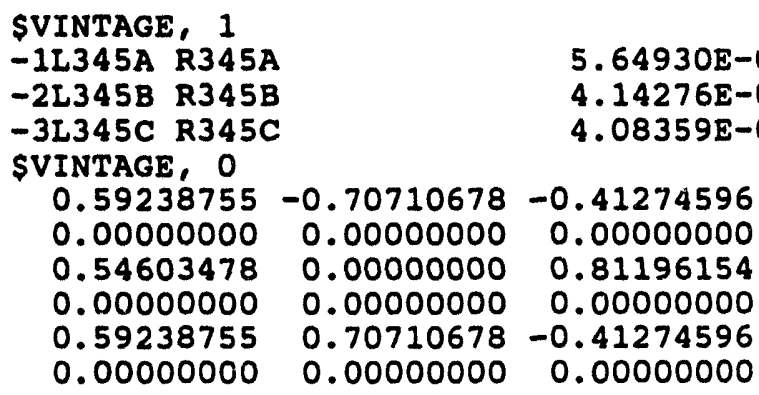




\section{Transformer saturation Routine (sample for T1):}

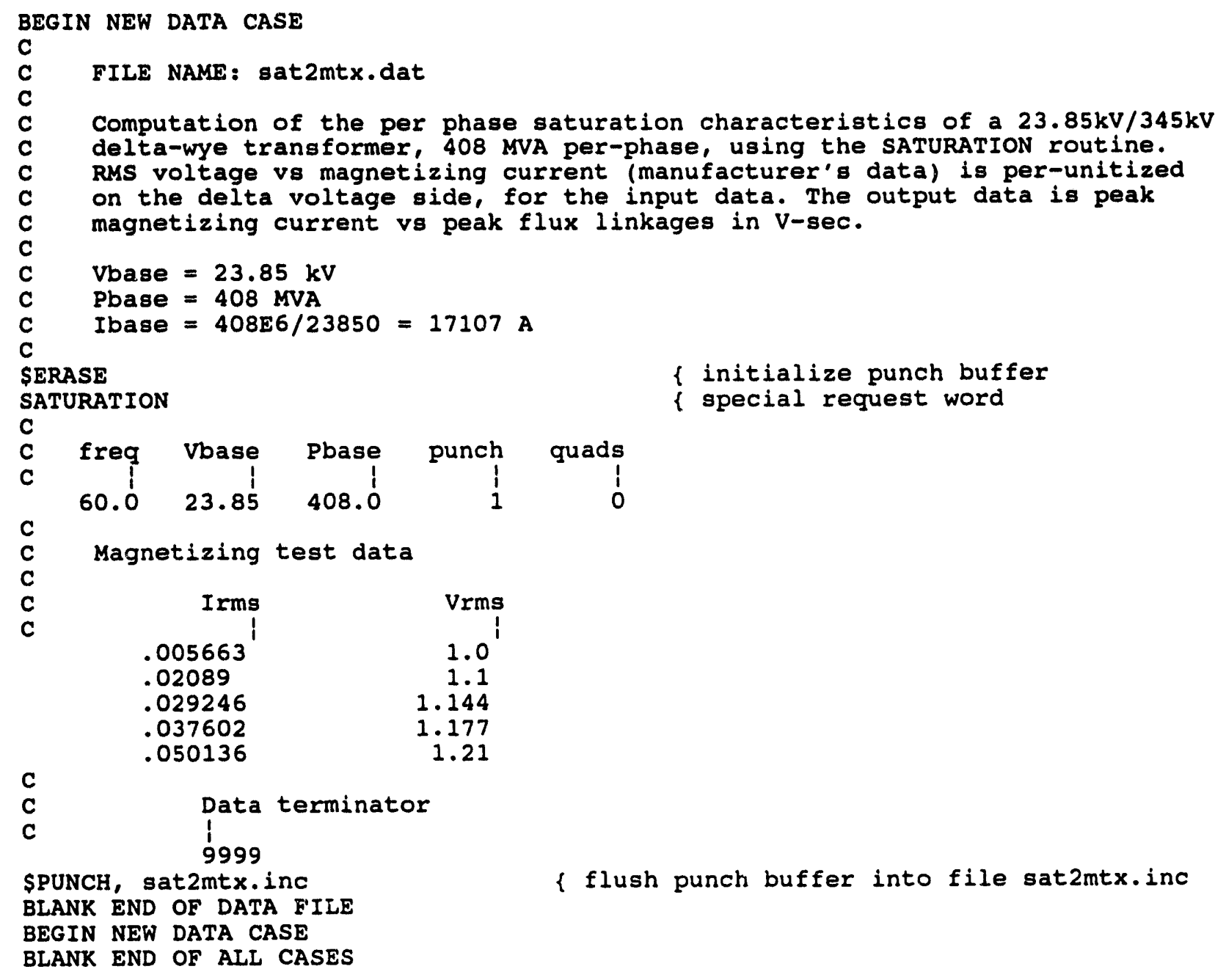

\section{Saturation Output Data:}
1. $37004029 \mathrm{E}+02$
8.79464078E+02
$1.13310186 \mathrm{E}+03$
1. $48958713 \mathrm{E}+03$
$2.05483294 \mathrm{E}+03$
$8.94689339 \mathrm{E}+01$
$9.84158273 \mathrm{E}+01$
$1.02352460 \mathrm{E}+02$
$1.05304935 \mathrm{E}+02$
1. $08257410 \mathrm{E}+02$

9999 
APPENDIX F:

SIMULATION OUTPUT WAVEFORMS

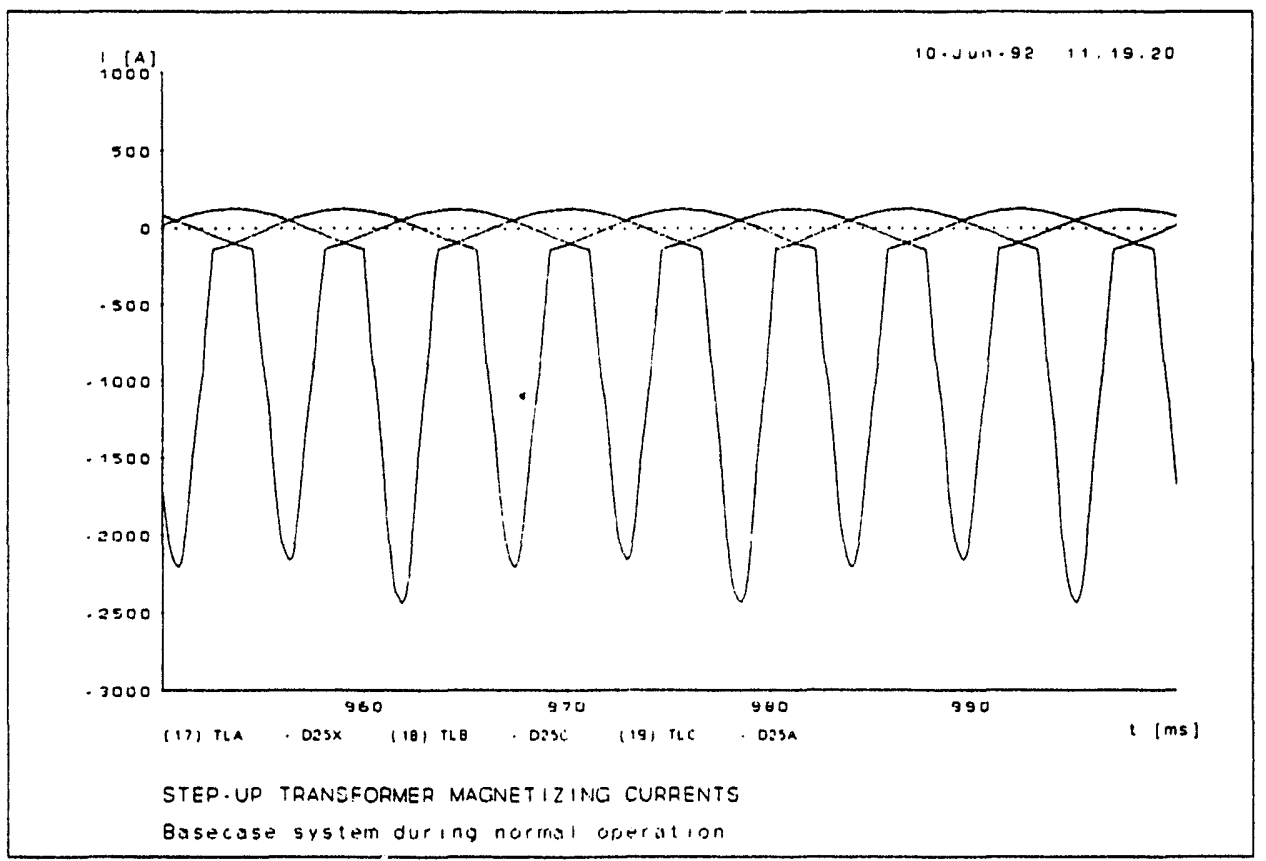

Fig. F-1. Step-up transformer magnetizing currents during normal operation with 100 A GIC.

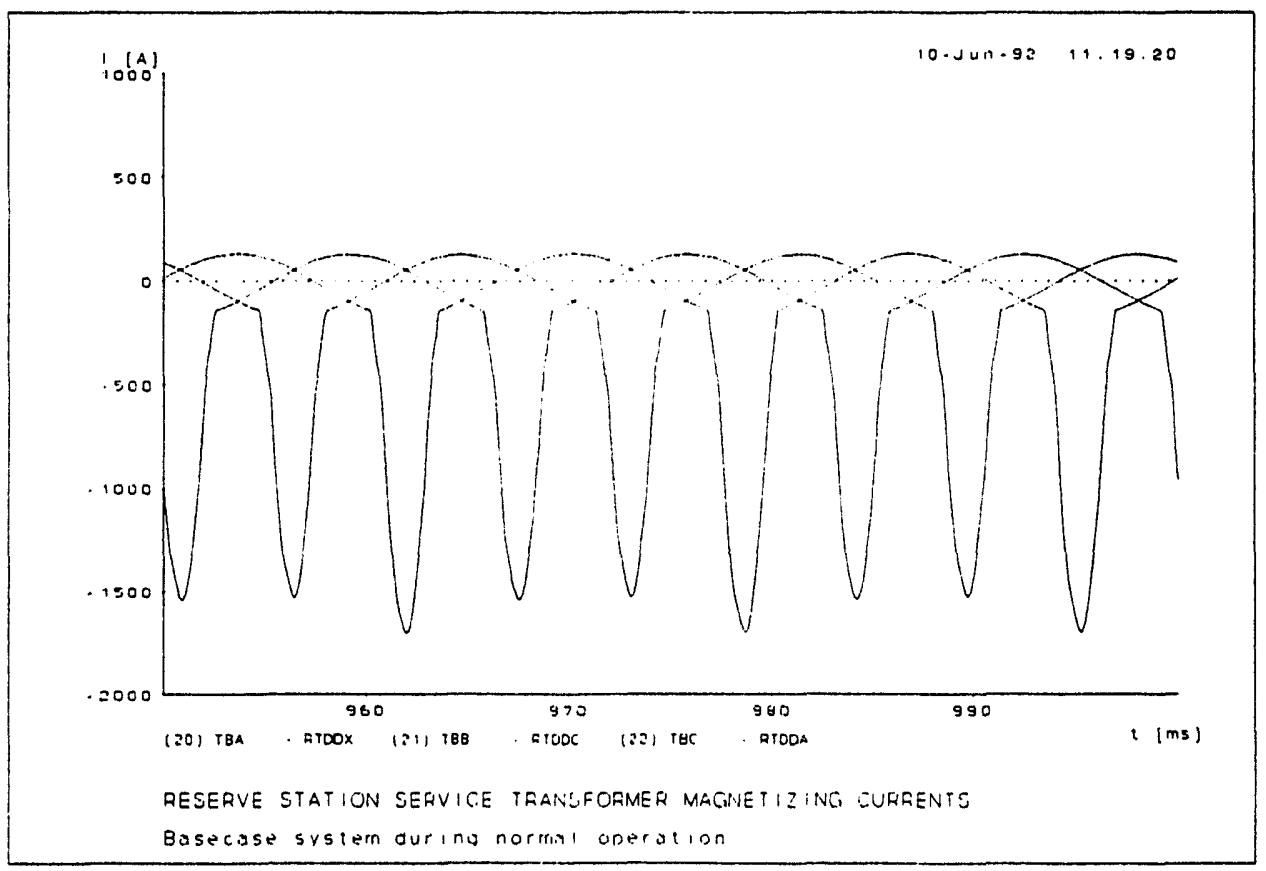

Fig. F-2. Reserve station service transformer magnetizing currents during normal operation with 100 A GIC.

$$
\text { F-1 }
$$




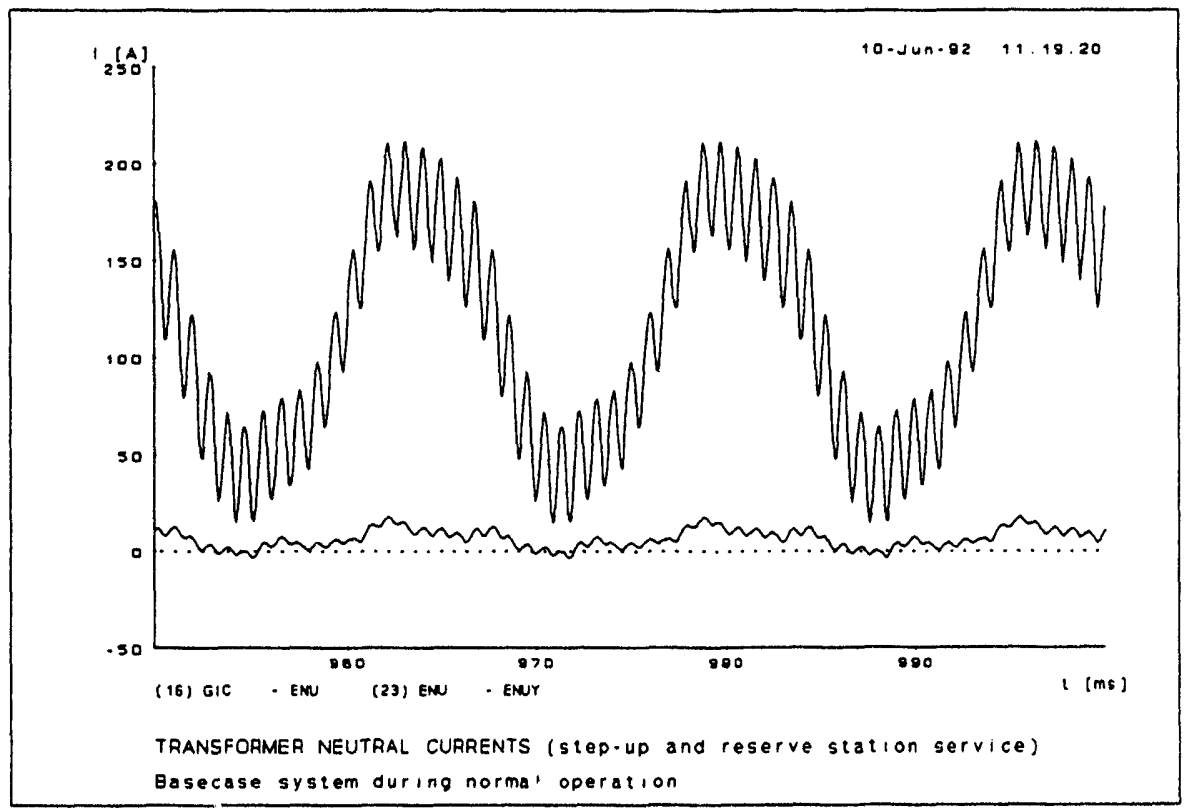

Fig. F-3. Neutral currents in step-up and reserve station service transformers during normal operation with basecase GIC.

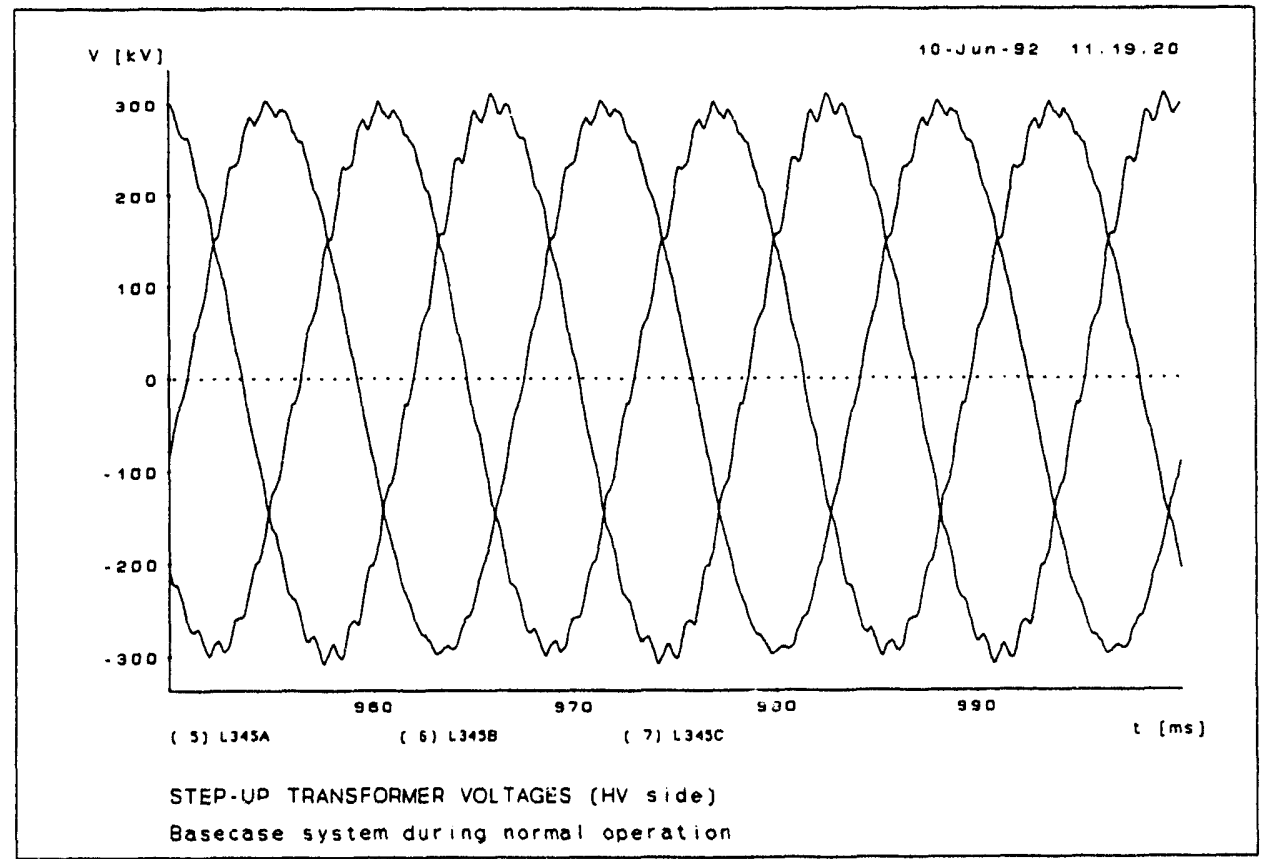

Fig. F-4. Step-up transformer voltages on $\mathrm{HV}$ side during normal operation with 100 A GIC. 

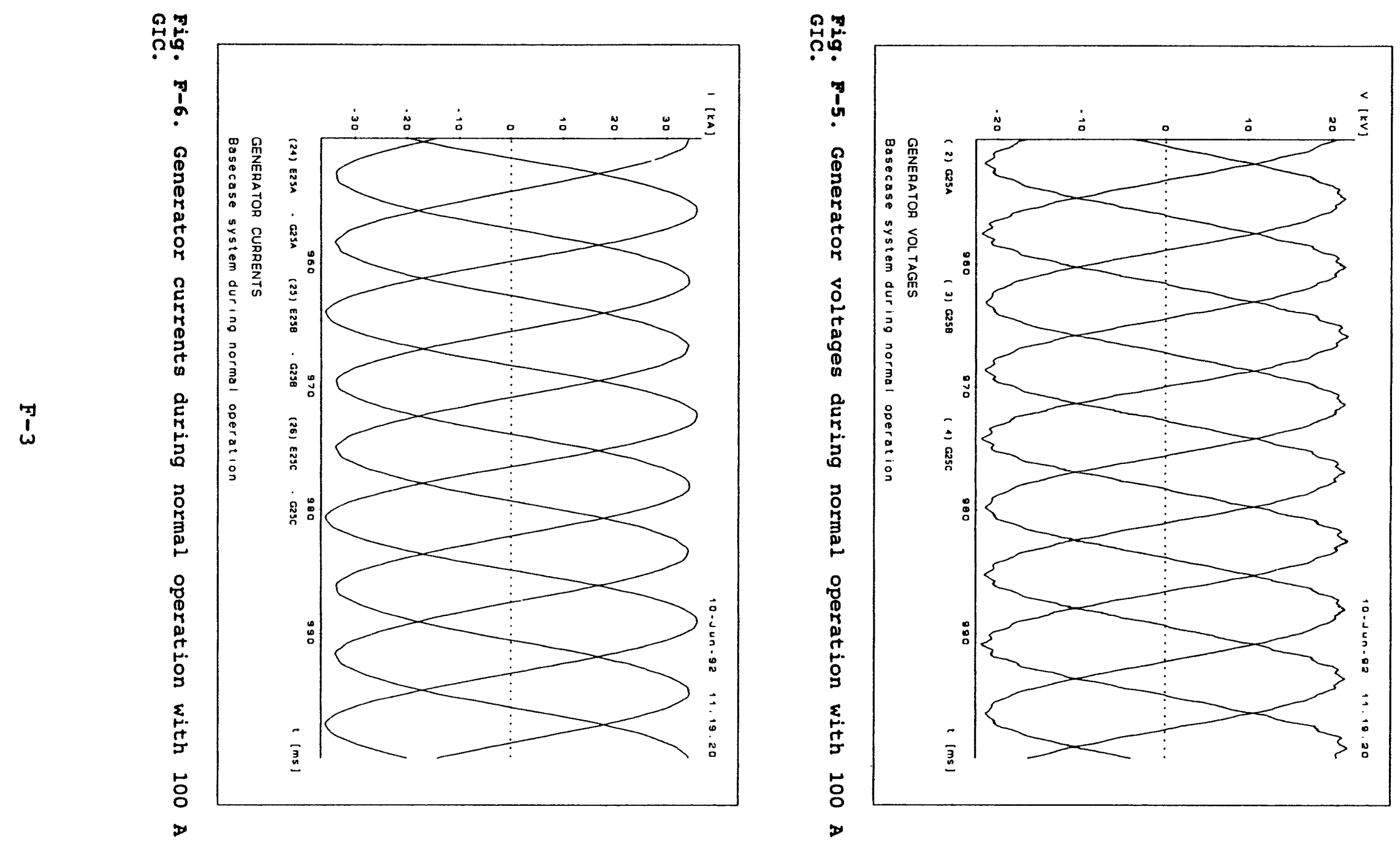


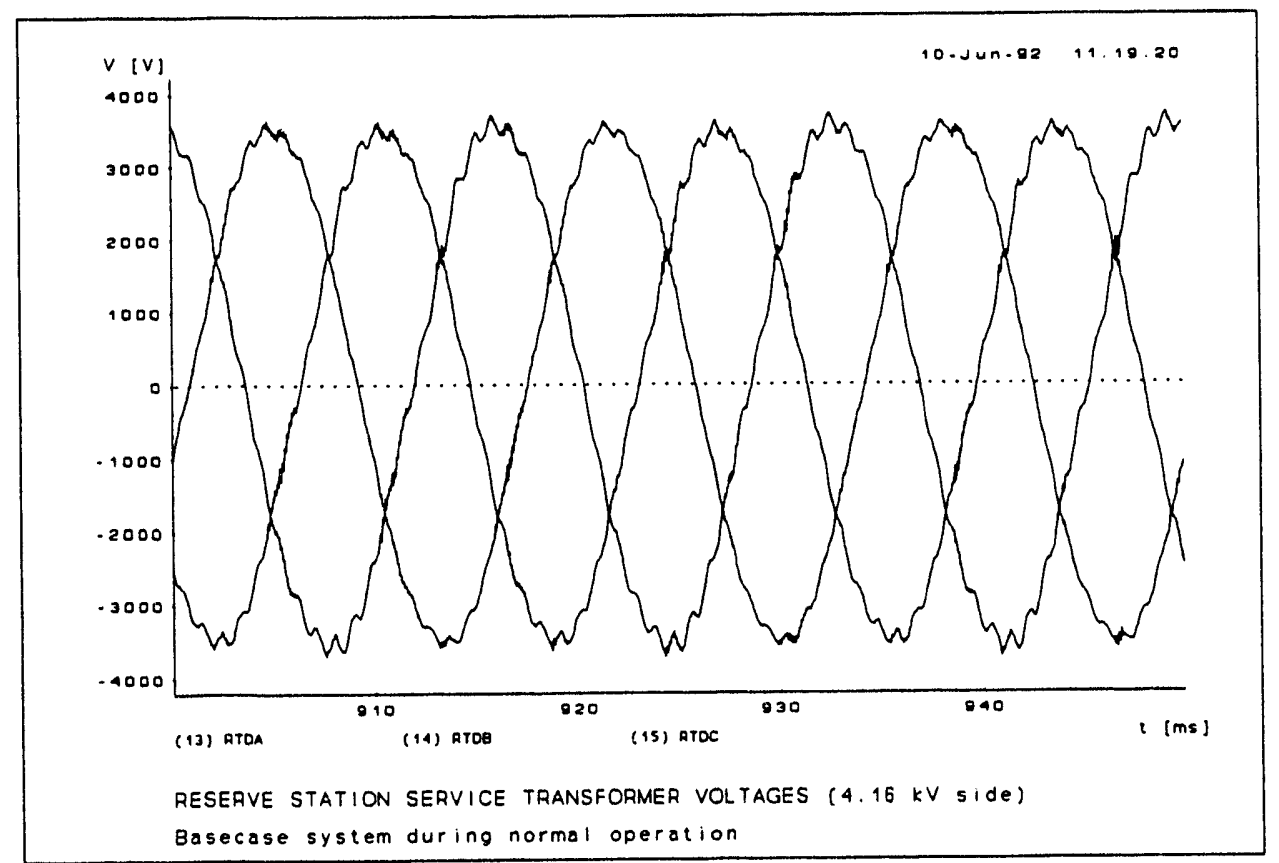

Fig. F-7. Reserve station service transformer voltages on $4.16 \mathrm{kV}$ side during normal operation with 100 A GIC.

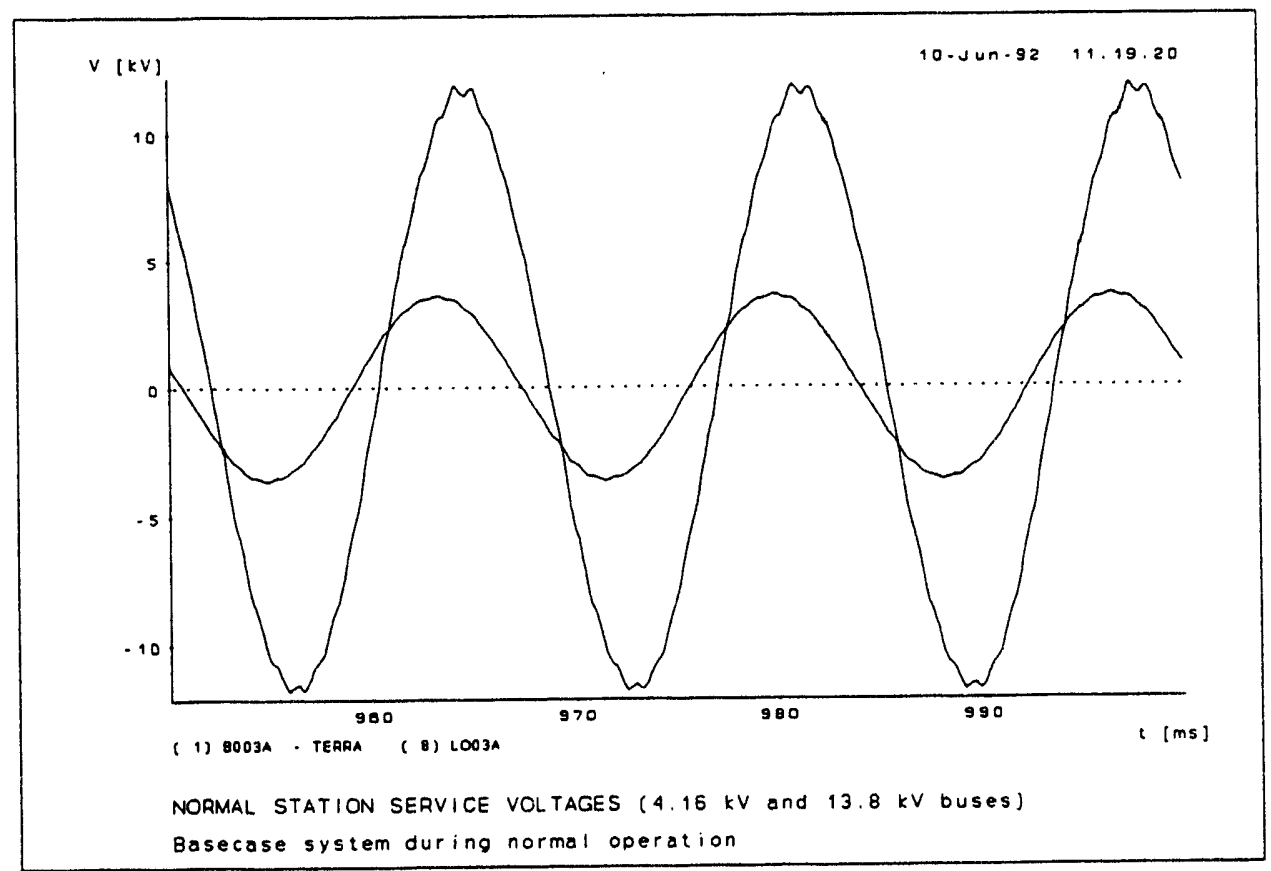

Fig. F-8. Normal station service voltages at $4.16 \mathrm{kV}$ and $13.8 \mathrm{kV}$ buses during normal operation with 100 A GIC. 

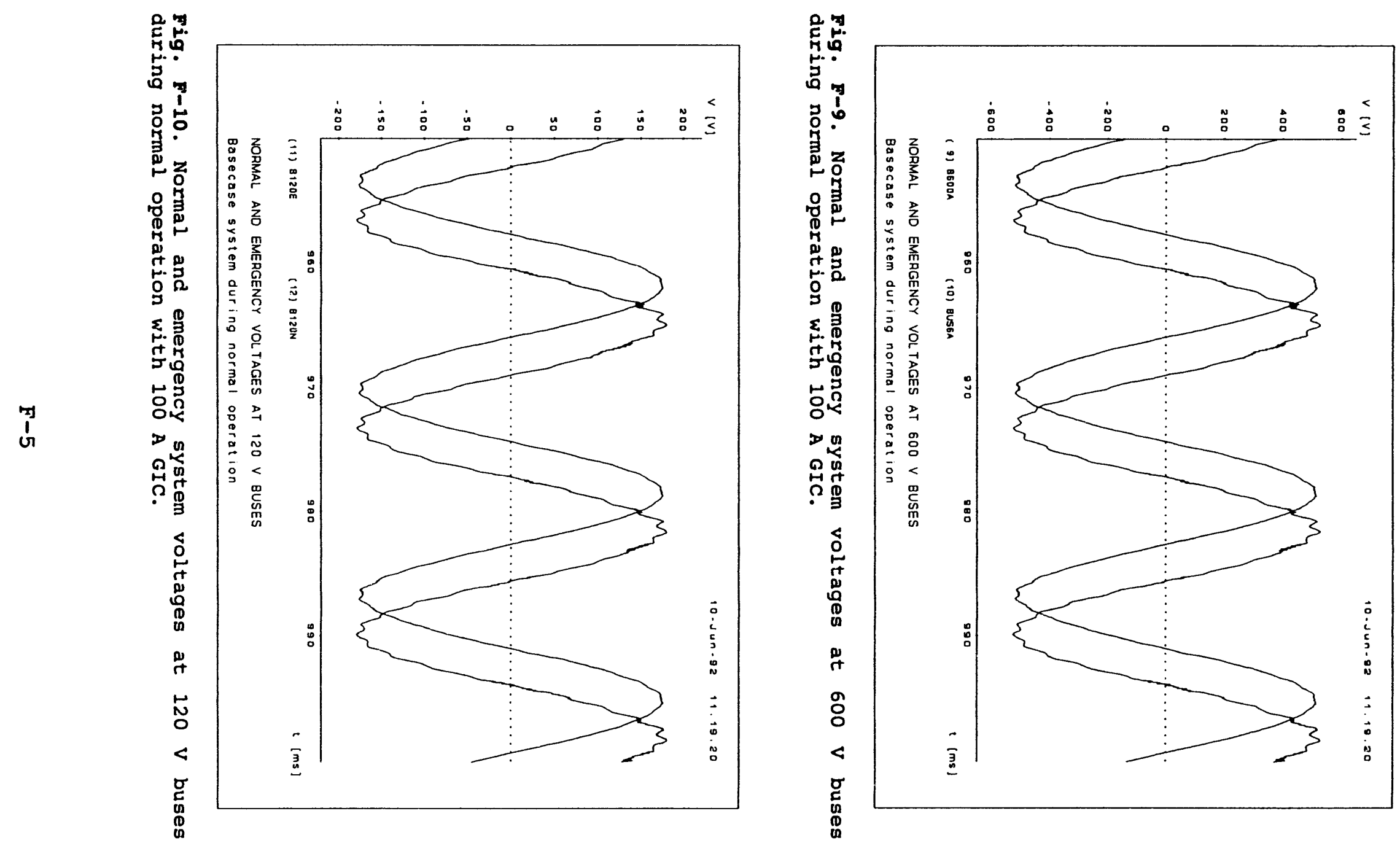


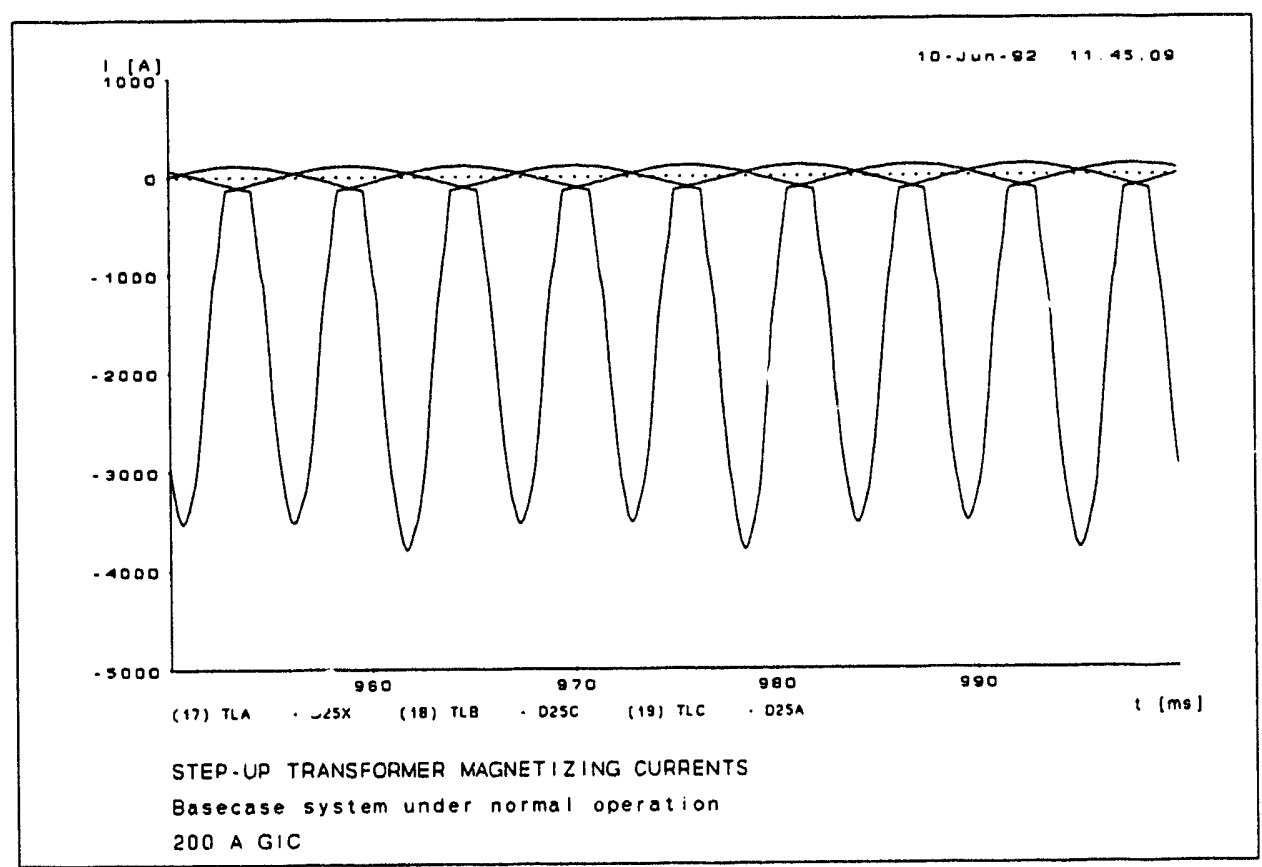

Fig. F-11. Step-up transformer magnetizing currents during normal operation with 200 A GIC.

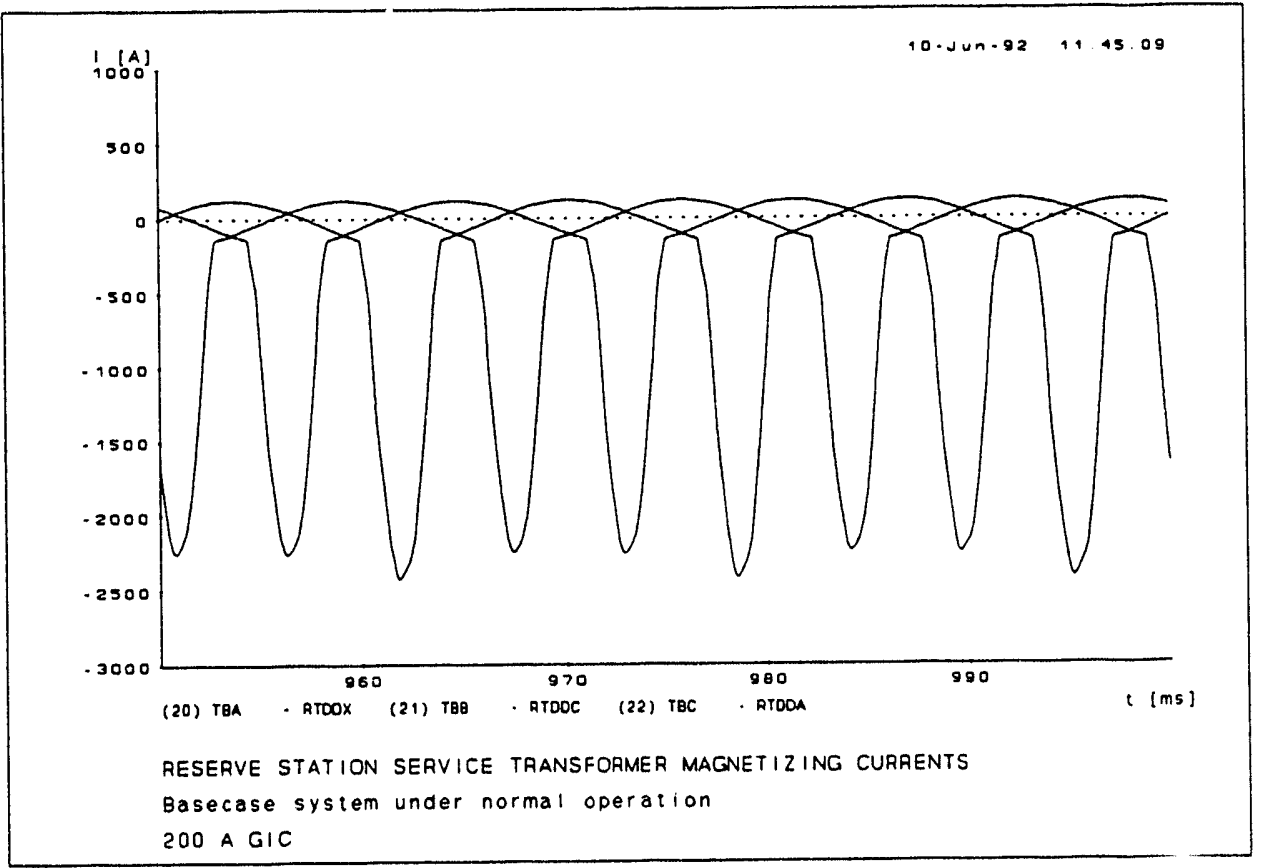

Fig. F-12. Reserve station service transformer magnetizing currents during normal operation with 200 A GIC. 


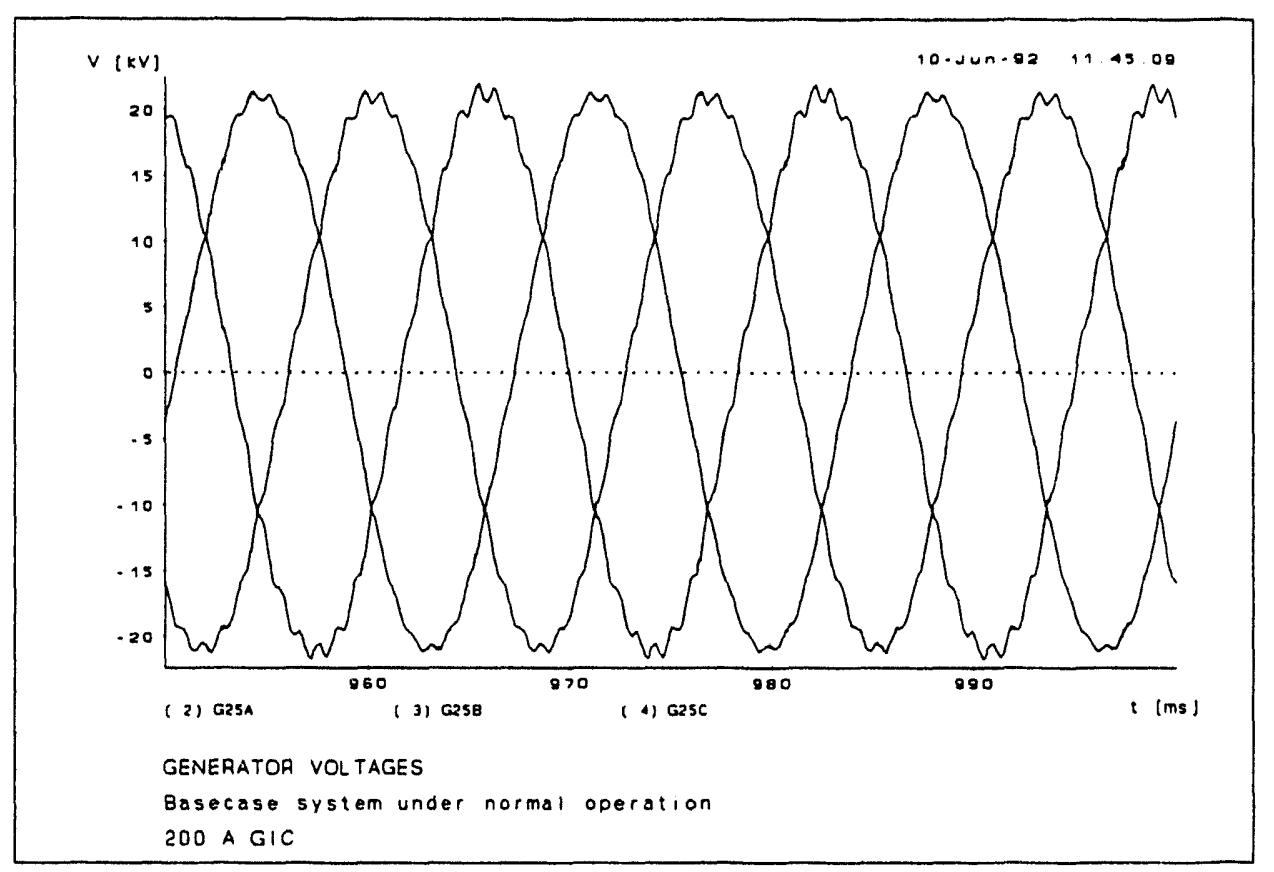

Fig. F-13. Generator voltages during normal operation with $200 \mathrm{~A}$ GIC.

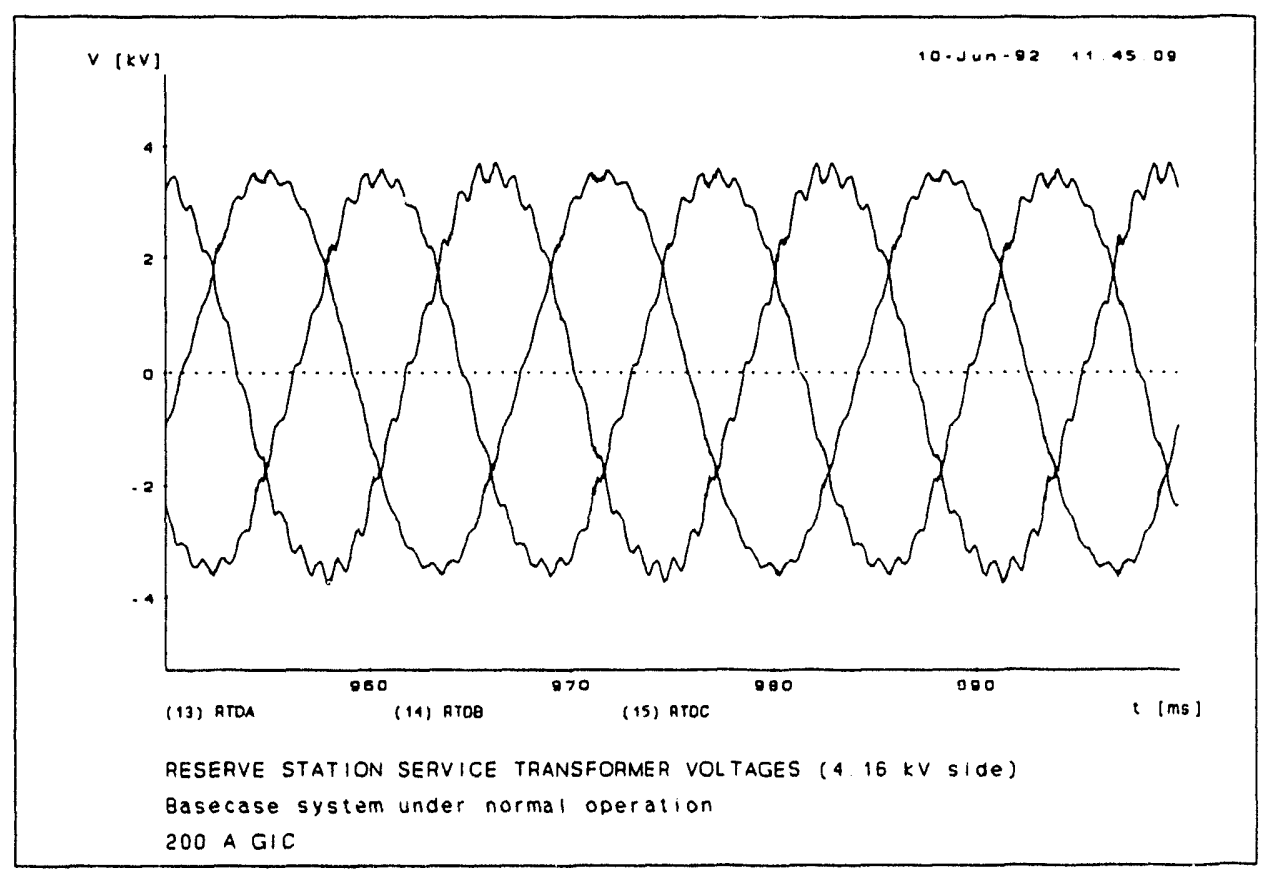

Fig. F-14. Reserve station service transformer voltages on $4.16 \mathrm{kV}$ side during normal operation with 200 A GIC. 


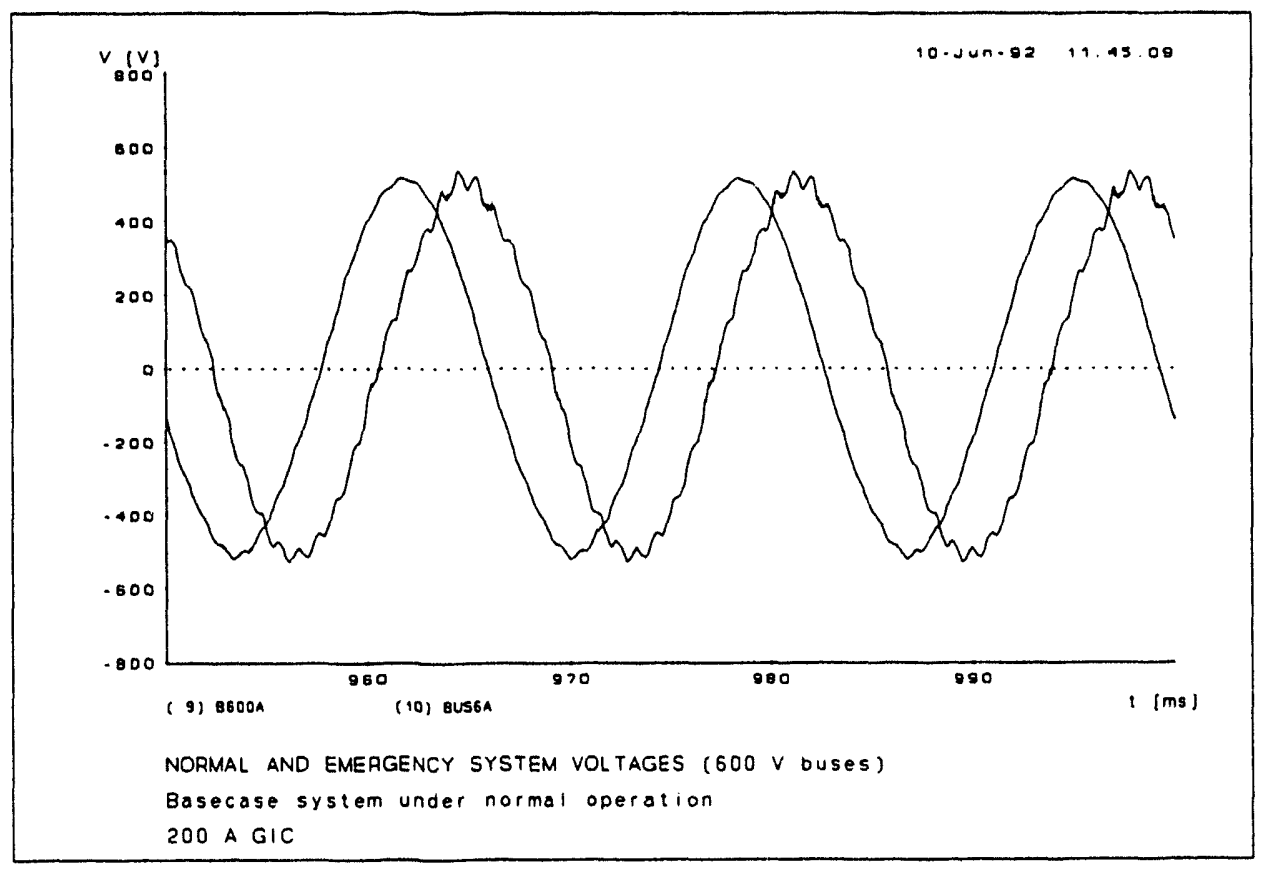

Fig. F-15. Normal and emergency system voltages at $600 \mathrm{~V}$ buses during normal operation with 200 A GIC.

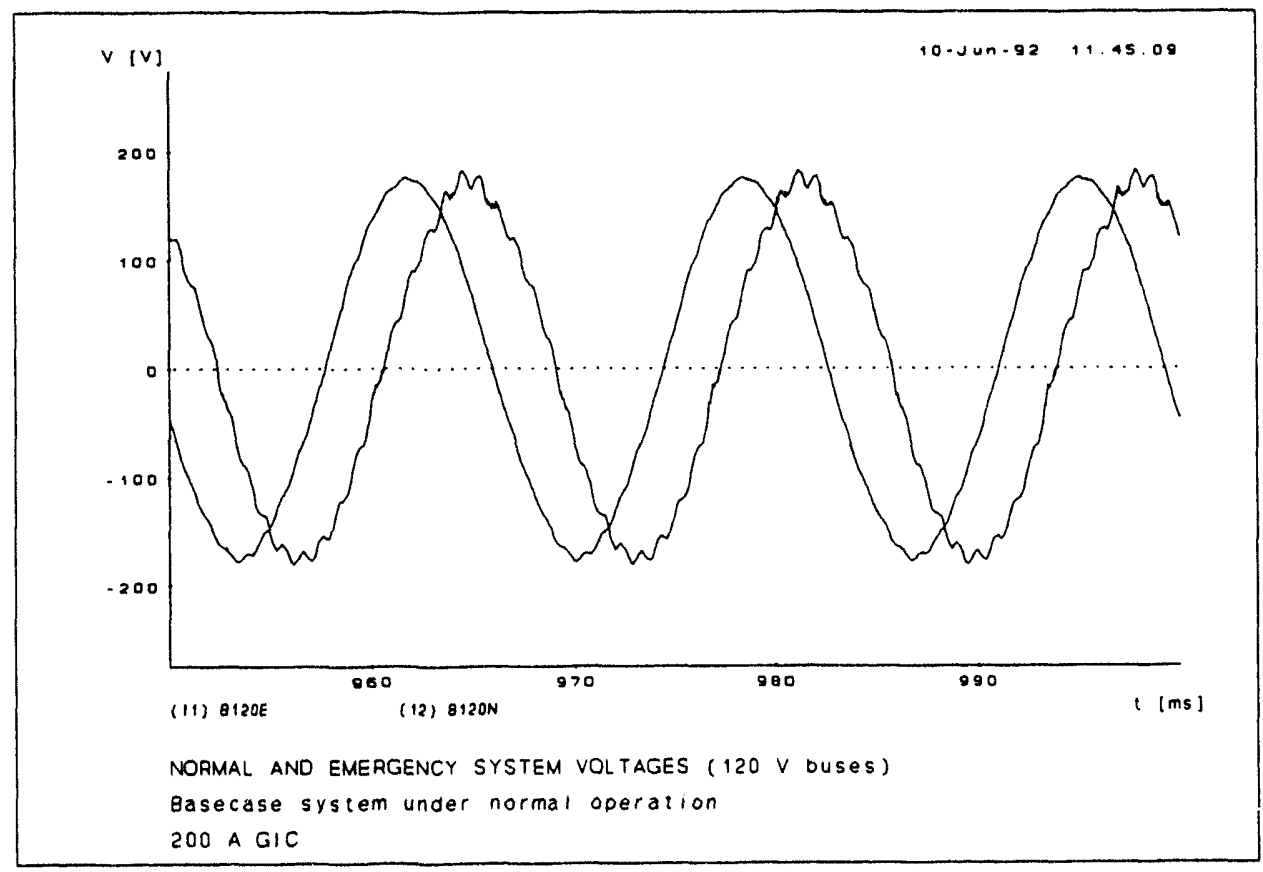

Fig. F-16. Normal and emergency system voltages at $120 \mathrm{~V}$ buses during normal operation with 200 A GIC. 


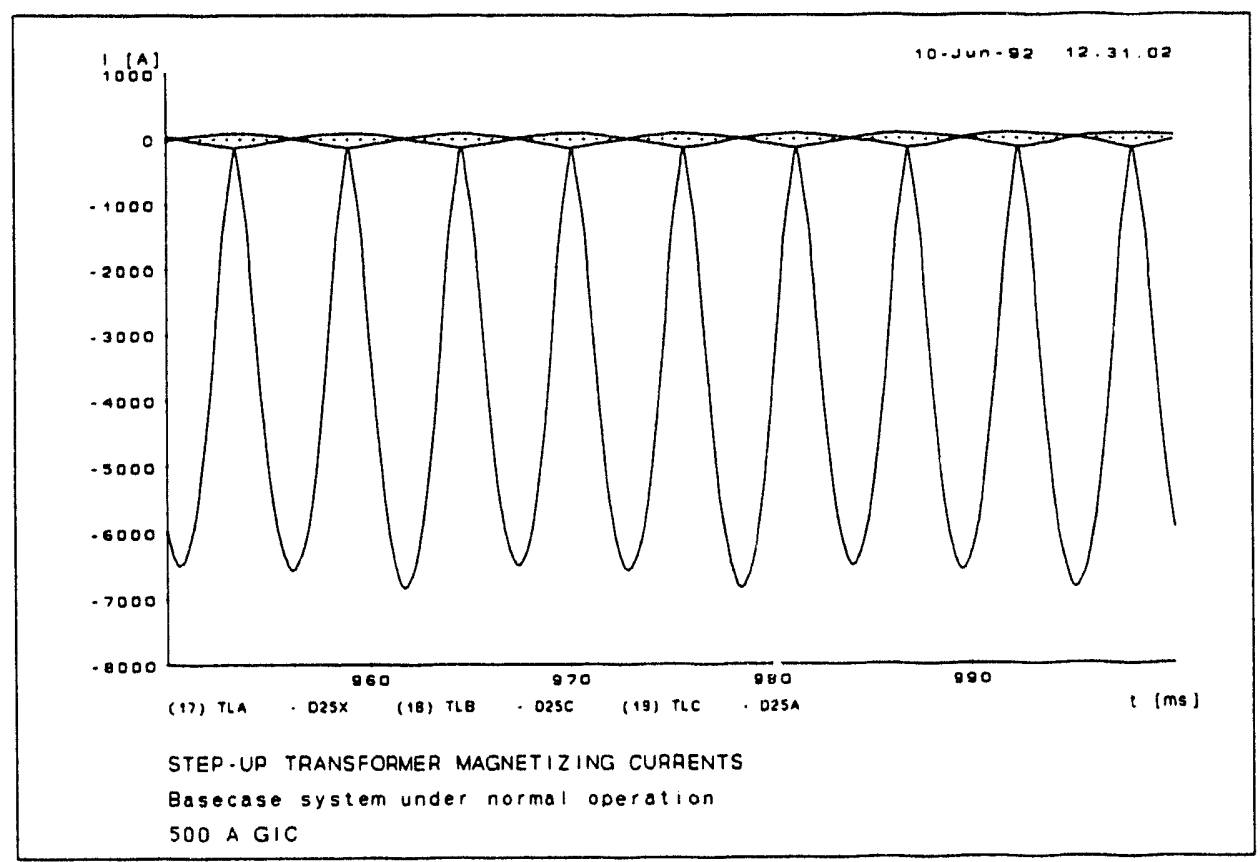

Fig. F-17. Step-up transformer magnetizing currents during normal operation with 500 A GIC.

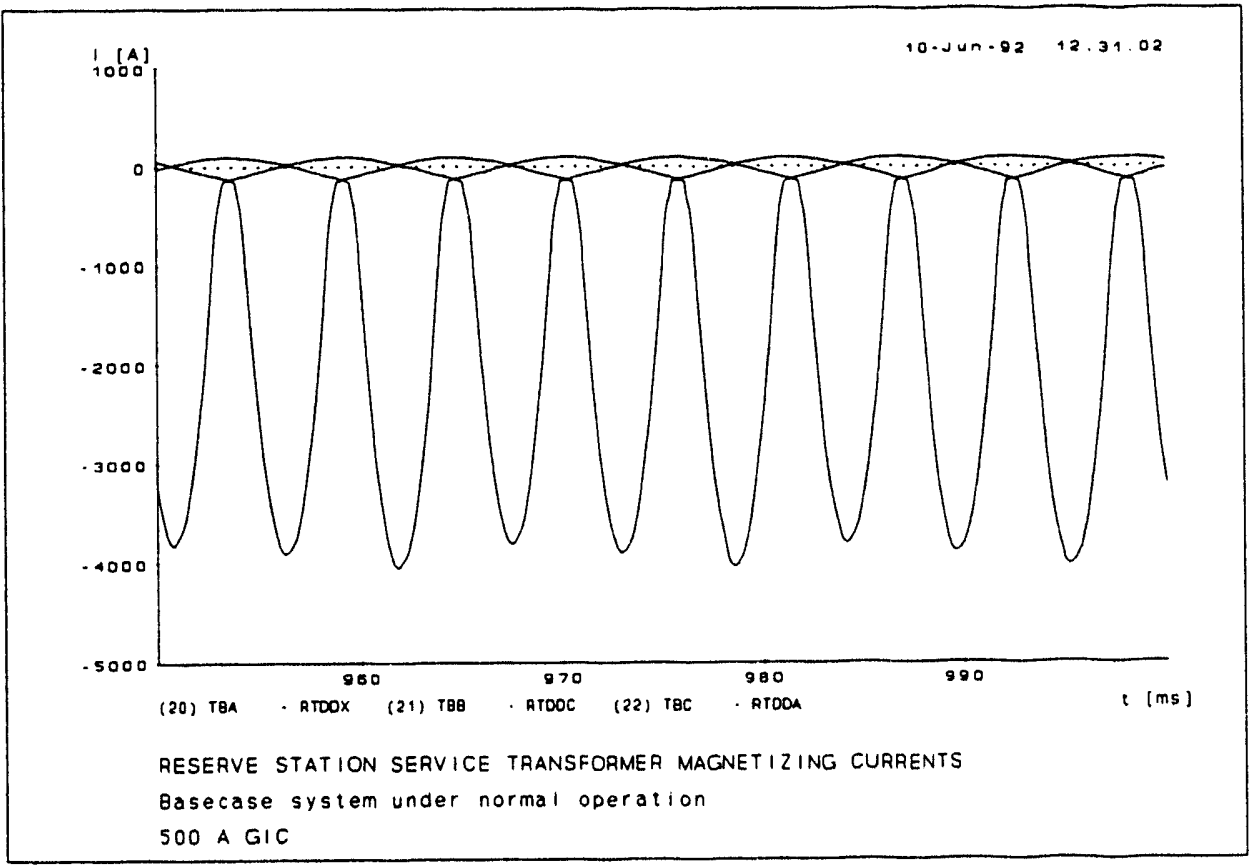

Fig. F-18. Reserve station service transformer magnetizing currents during normal operation with 500 A GIC. 


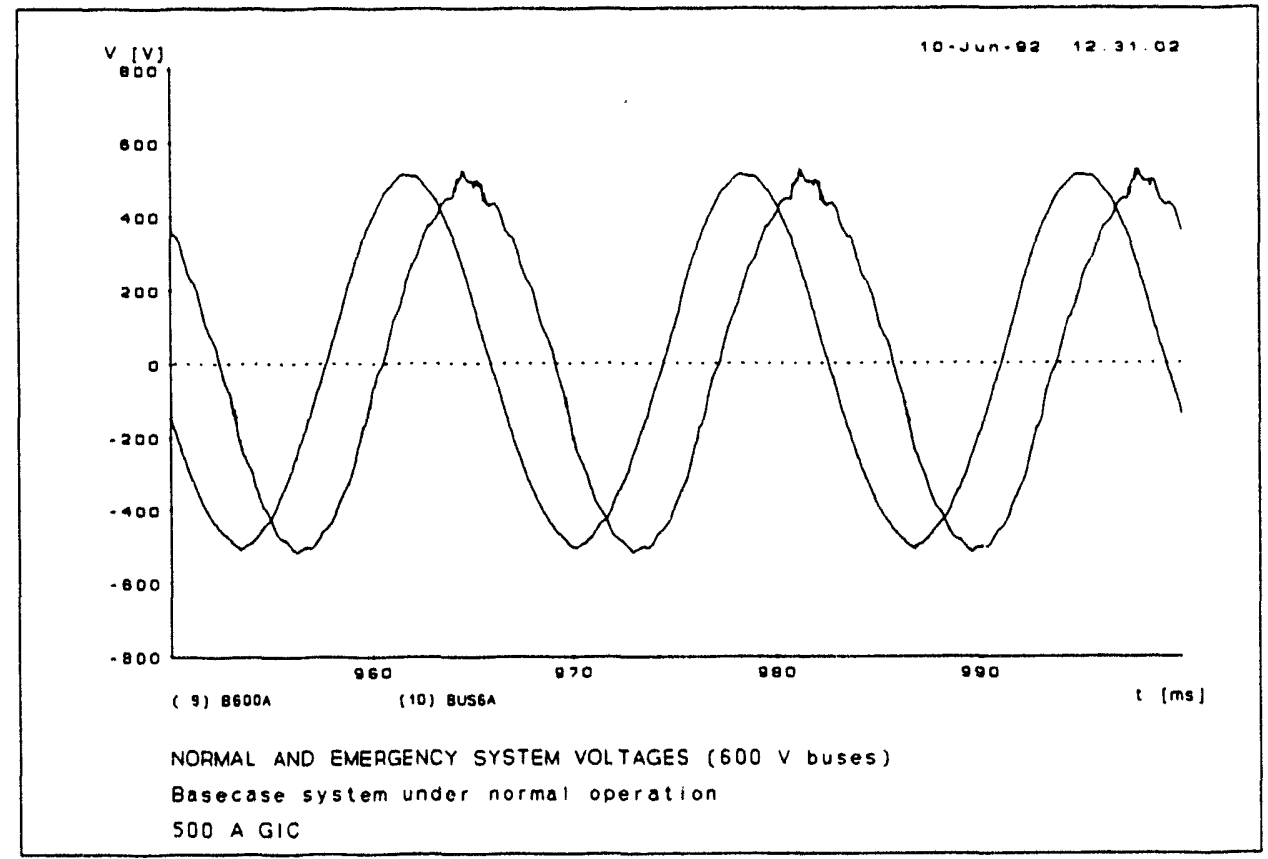

Fig. F-19. Normal and emergency system voltages at $600 \mathrm{~V}$ buses during normal operation with 500 A GIC.

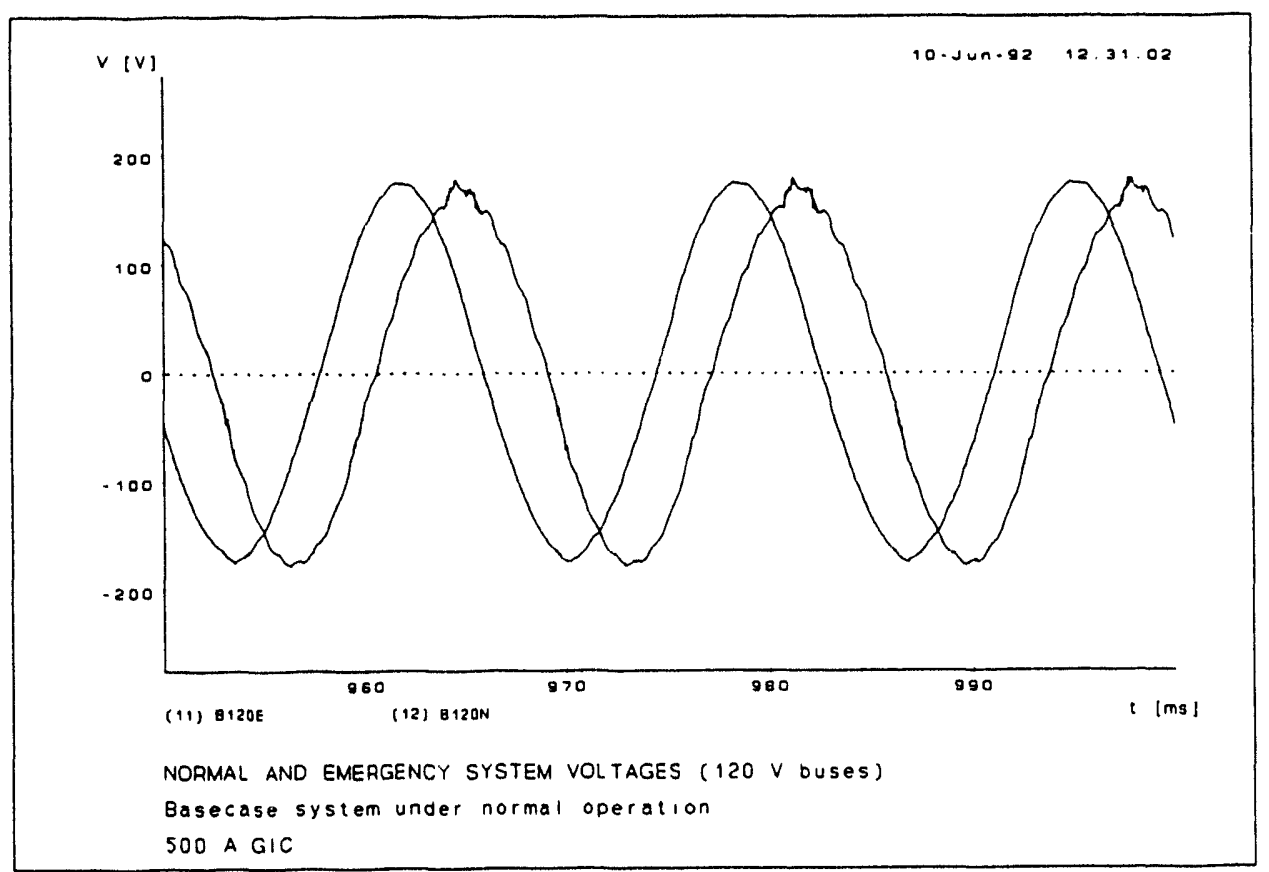

Fig. F-20. Normal and emergency system voltages at $120 \mathrm{~V}$ buses during normal operation with 500 A GIC. 


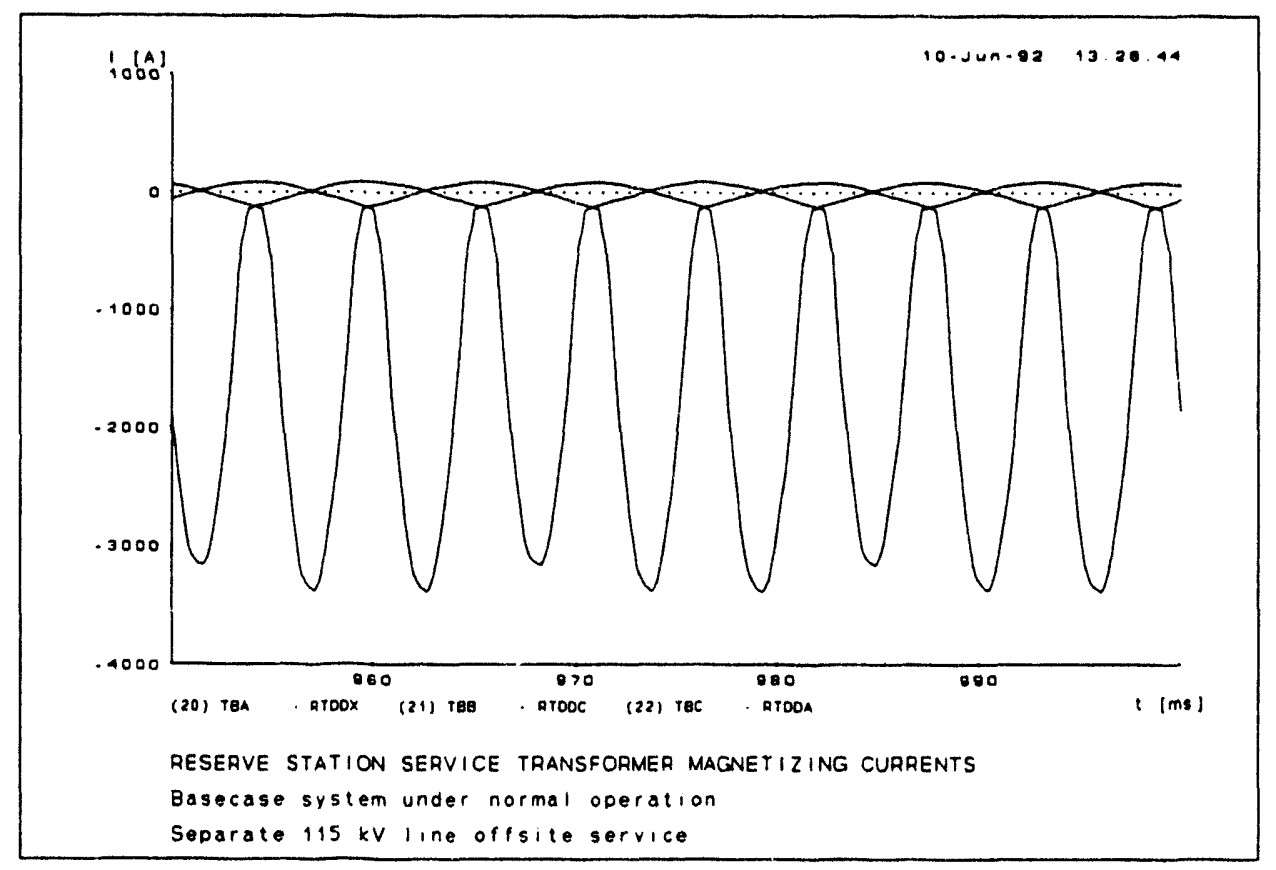

Fig. F-21. Reserve station service transformer magnetizing currents during normal operation with $100 \mathrm{~A}$ GIC and separate $115 \mathrm{kV}$ line.

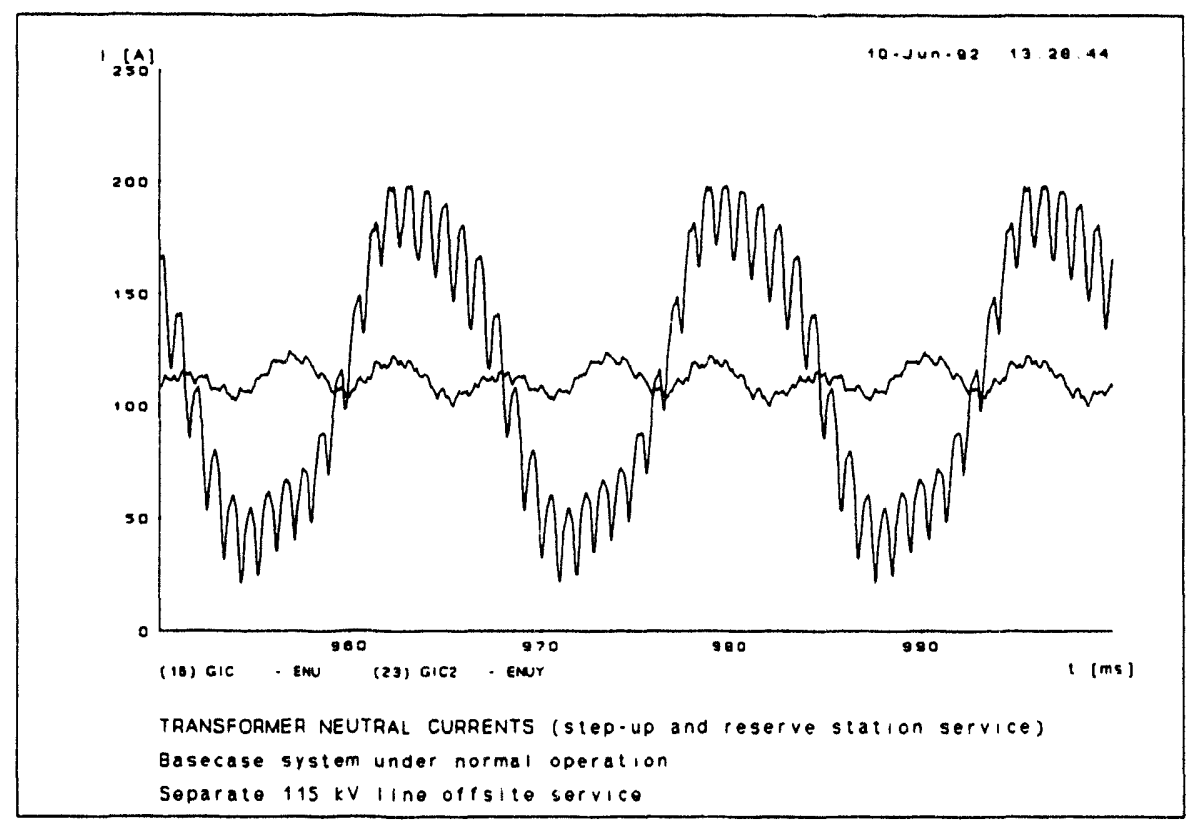

Fig. F-22. Neutral currents in step-up and reserve station service transformers during normal operation with separate $115 \mathrm{kV}$ line. 


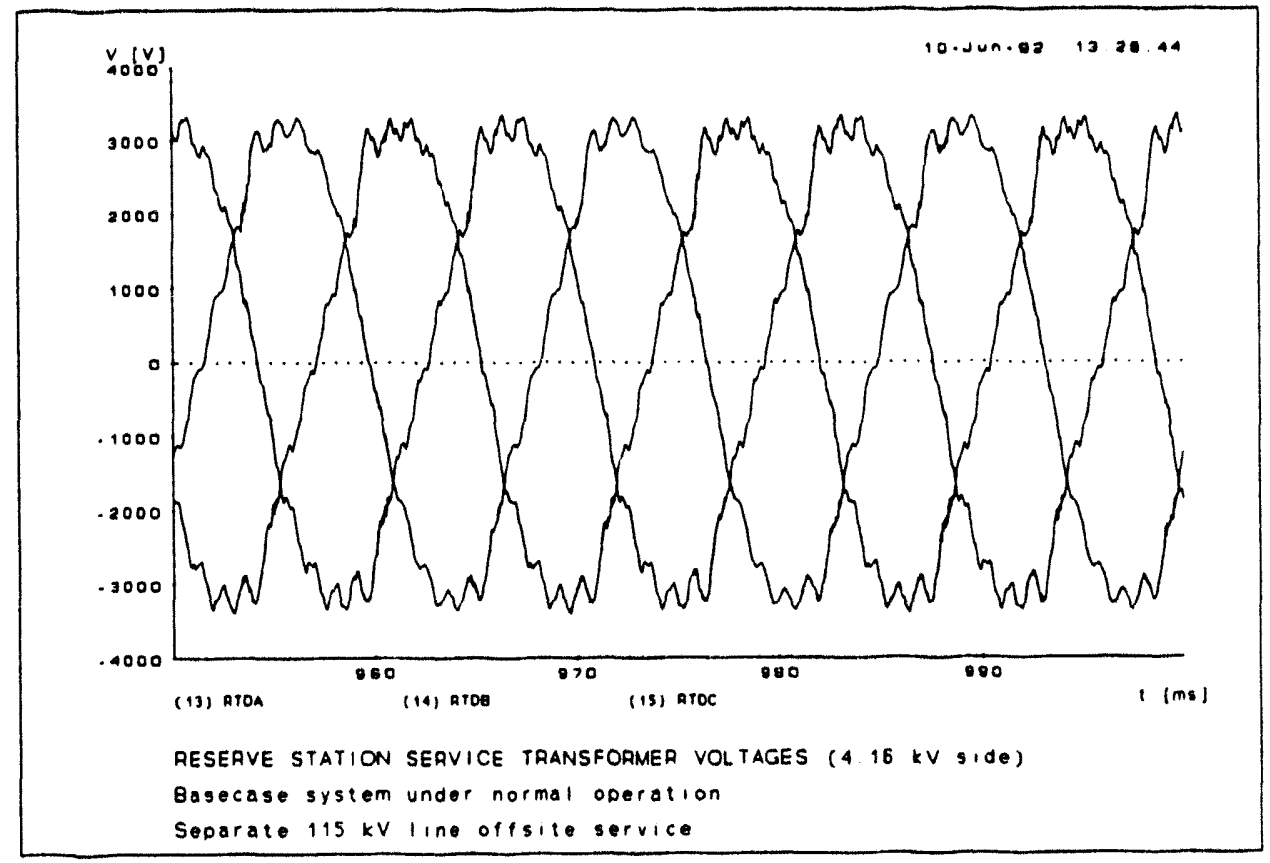

Fig. F-23. Reserve station service transformer voltages on $4.16 \mathrm{kV}$ side during normal operation with separate $115 \mathrm{kV}$ line.

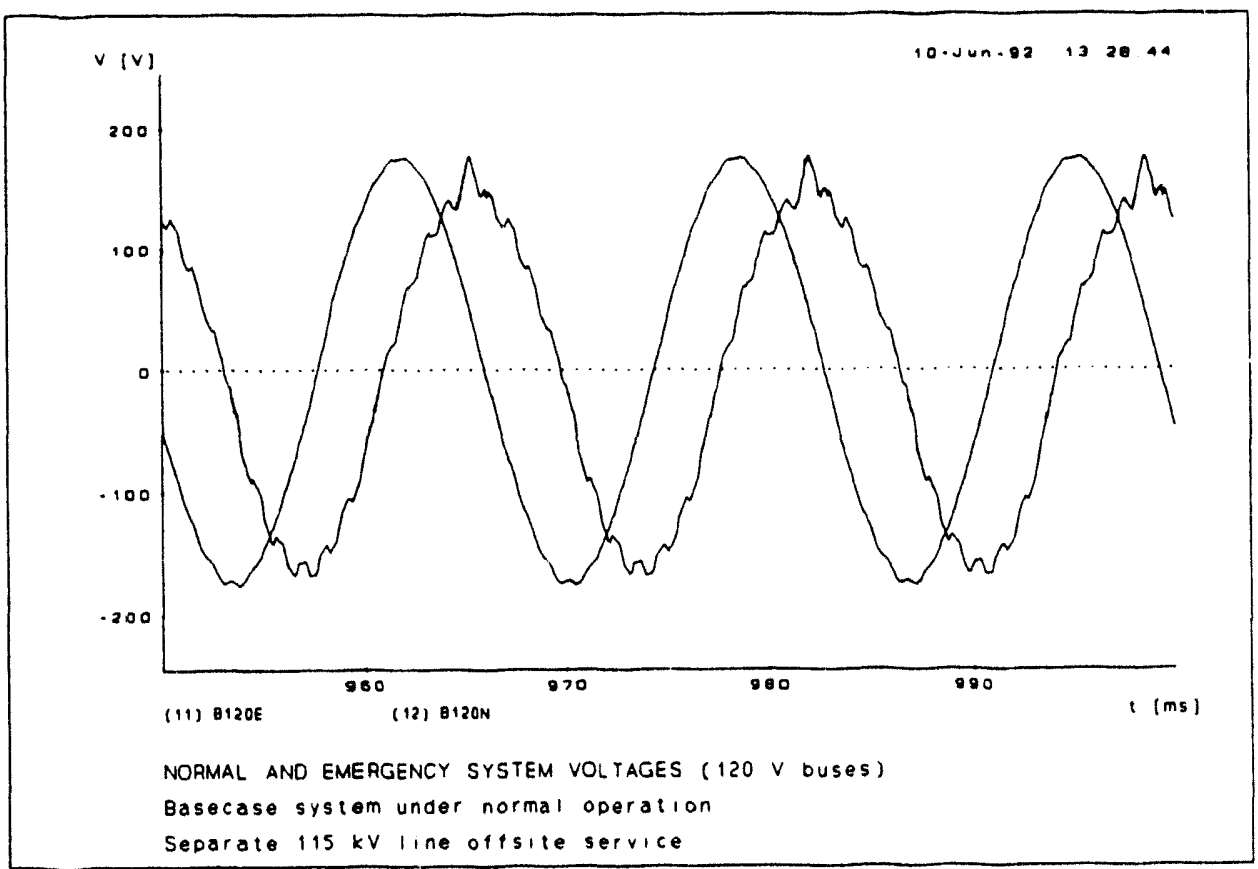

Fig. F-24. Normal and emergency system voltages at $120 \mathrm{~V}$ buses during normal operation with separate $115 \mathrm{kV}$ line. 


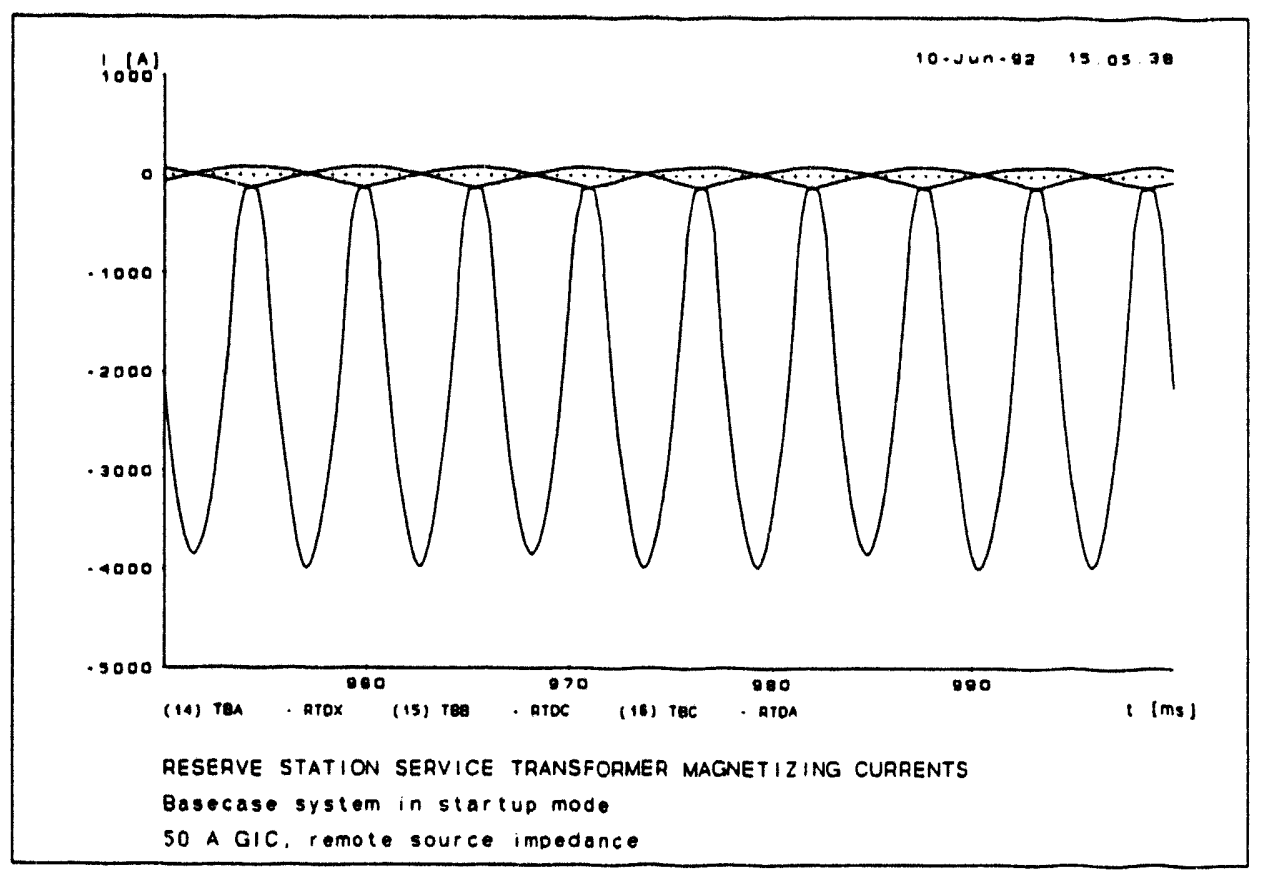

Fig. F-25. Reserve station service transformer magnetizing currents during startup mode with 50 A GIC and remote source impedance.

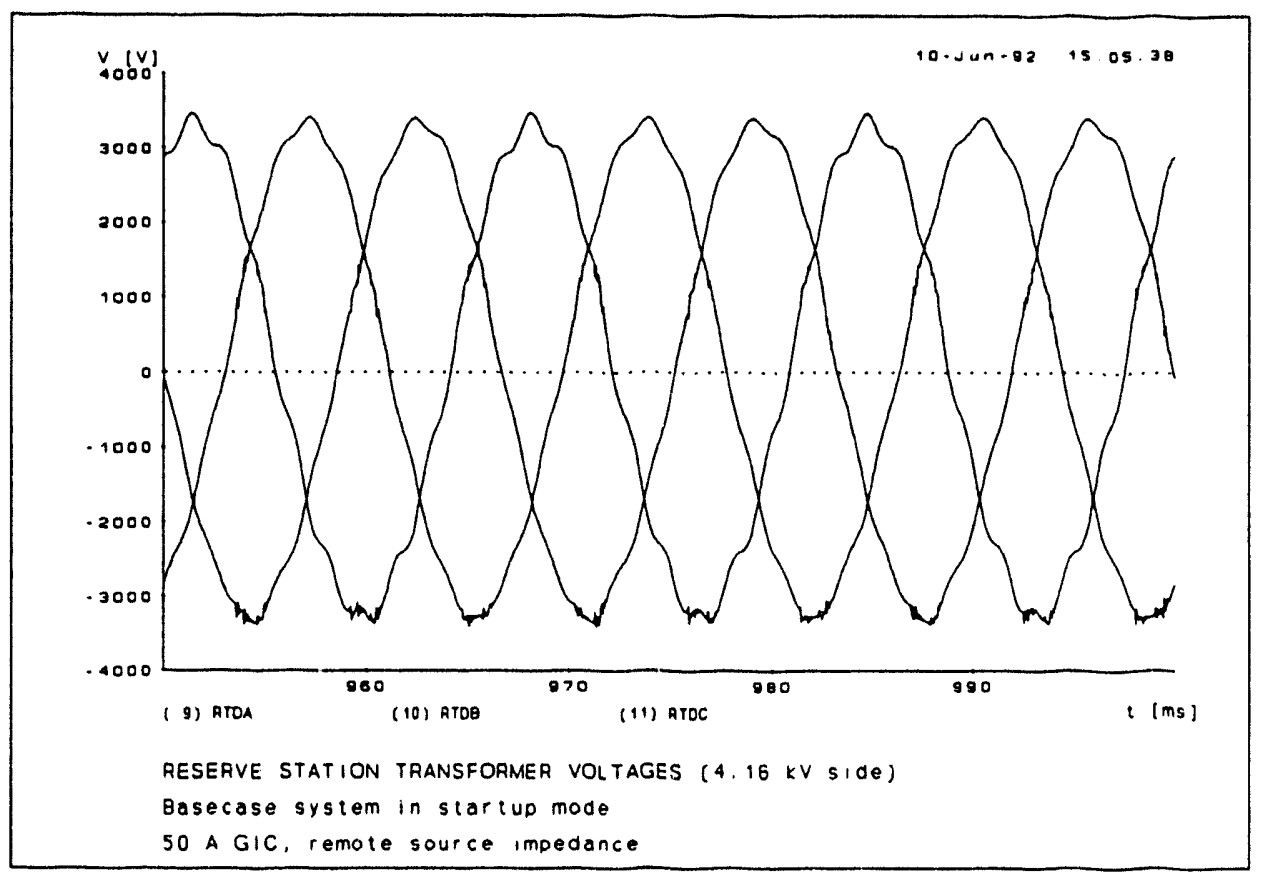

Fig. F-26. Reserve station service transformer voltages on $4.16 \mathrm{kV}$ side during startup mode with 50 A GIC and remote source impedance. 


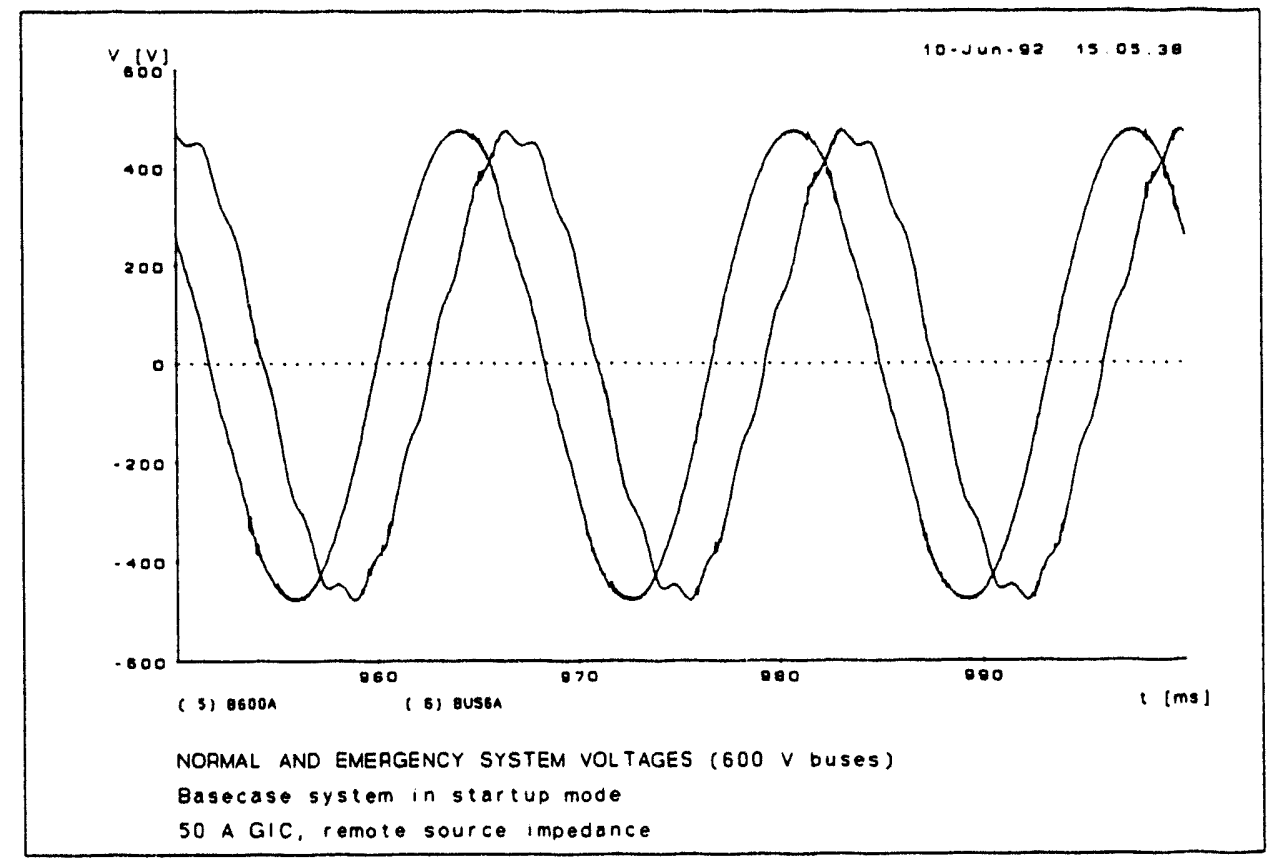

Fig. F-27. Normal and emergency system voltages at $600 \mathrm{~V}$ buses during startup mode with 50 A GIC and remote system impedance.

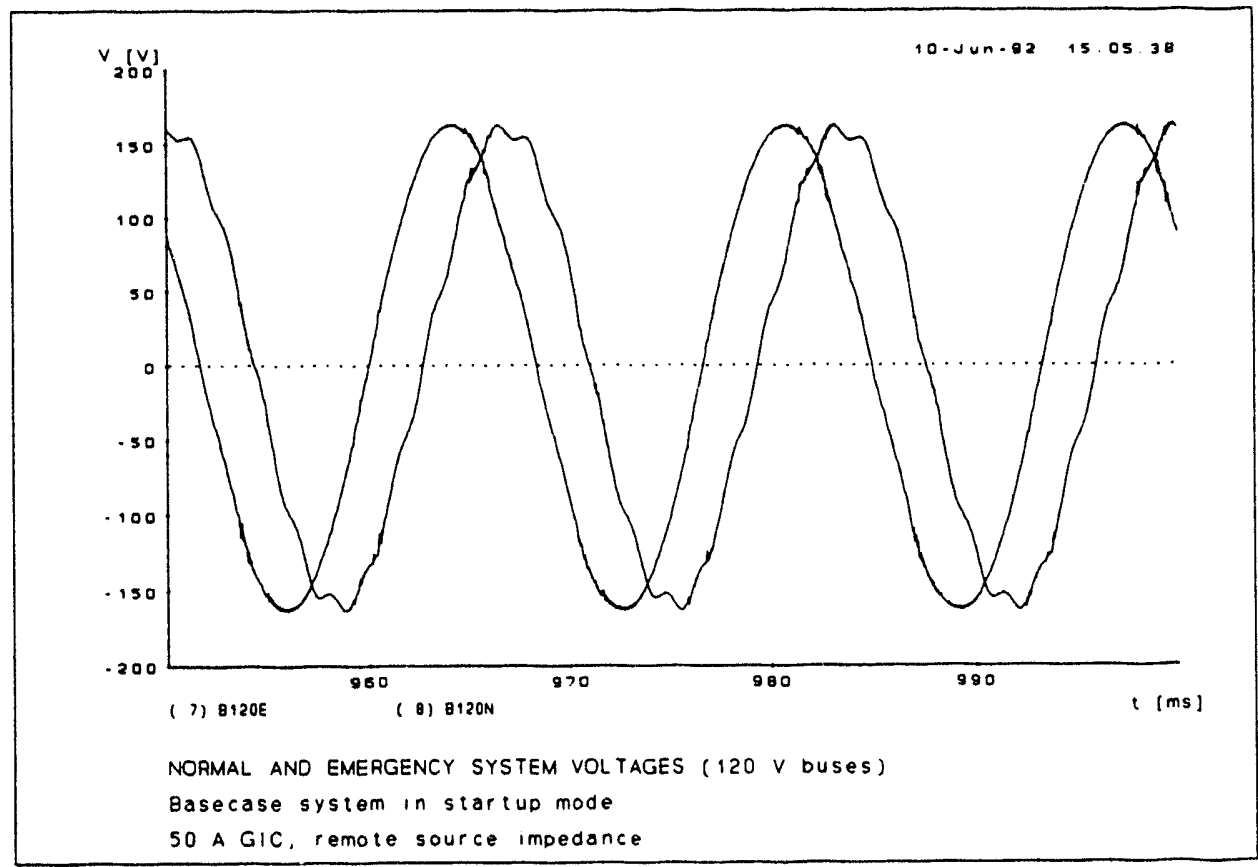

Fig. F-28. Normal and emergency system voltages at $120 \mathrm{~V}$ buses during startup mode with $50 \mathrm{~A}$ GIC and remote source impedance. 


\section{APPENDIX G: Frequency Scan Analysis}

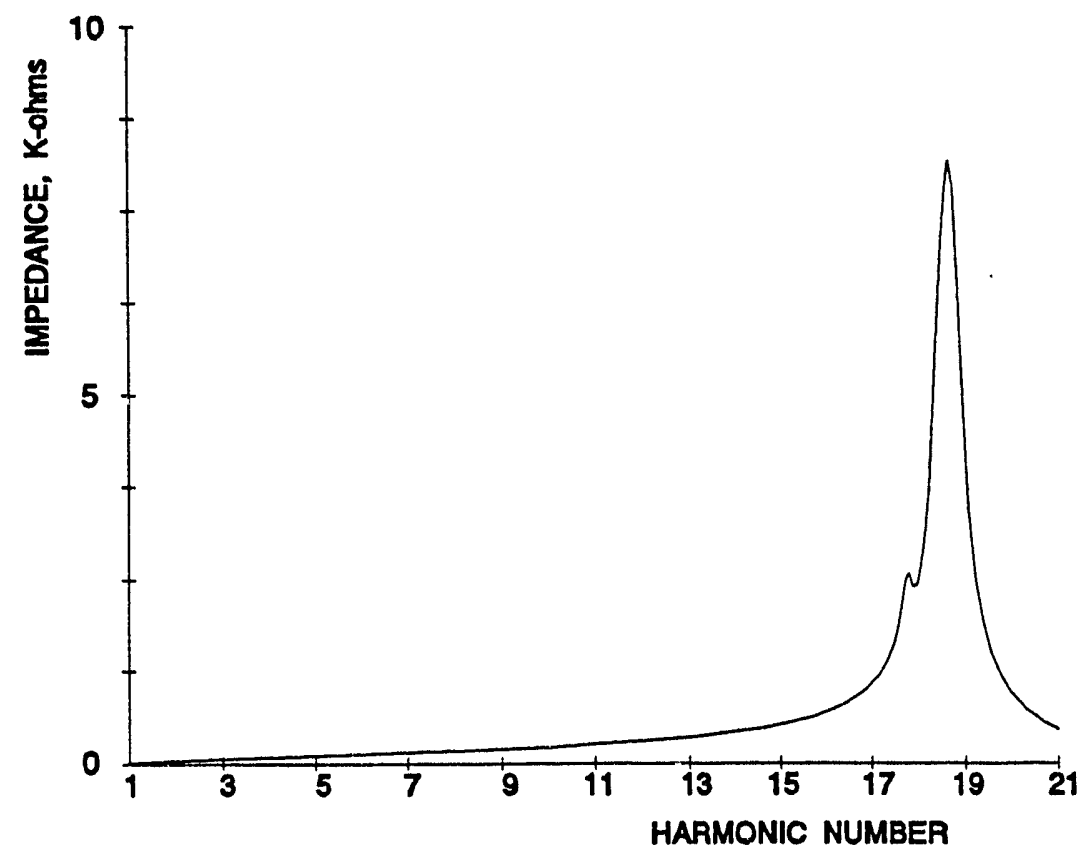

Fig. G-1. Transmission line impedance function for normal station configuration and nominal system parameters.

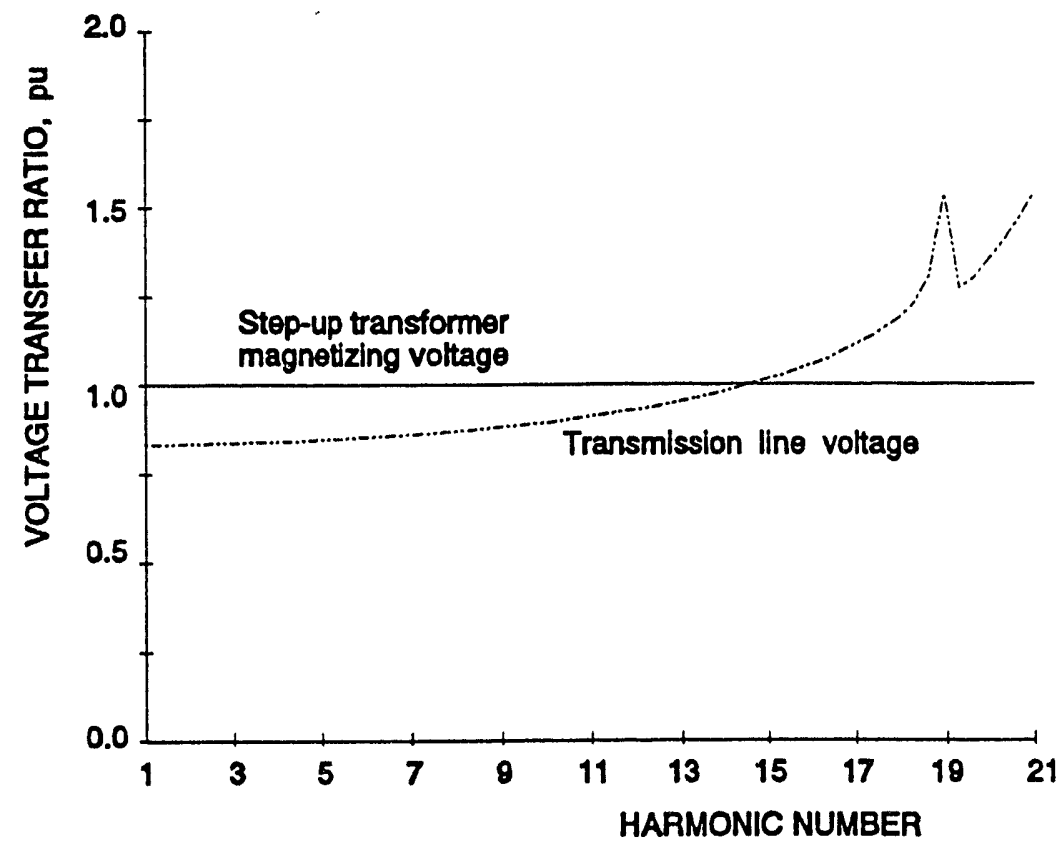

Fig. G-2. Transfer ratio of local transmission line voltage to step-up transformer voltage.

$$
\text { G-1 }
$$




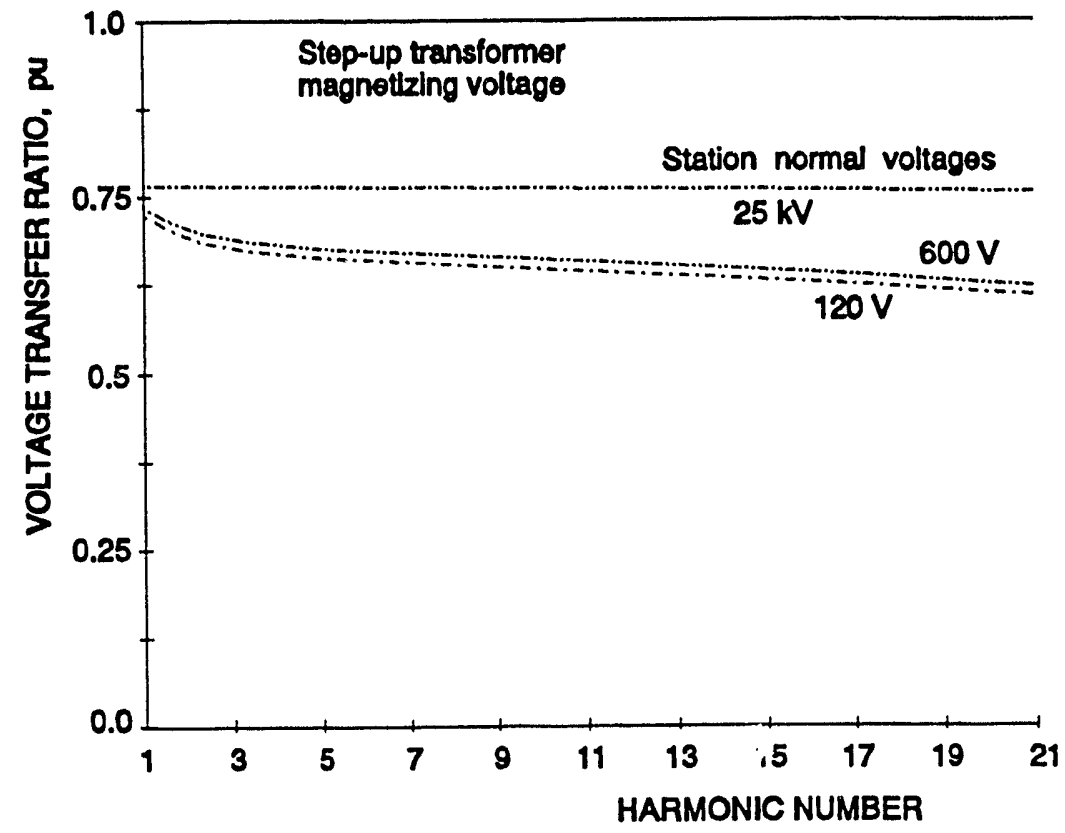

Fig. G-3. Transfer ratio of nonsafety-related distribution voltages to transformer excitation voltage for normal station configuration and nominal system parameters.

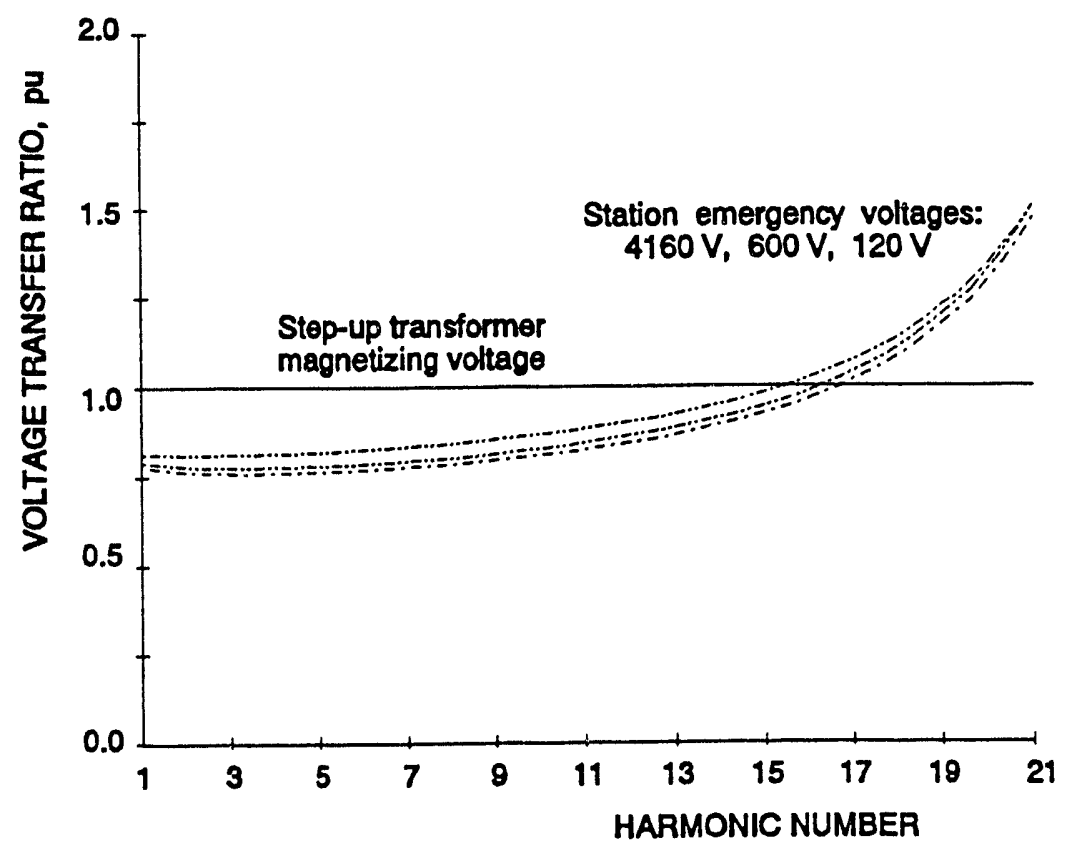

Fig. G-4. Transfer ratio of safety-related distribution voltages to transformer excitation voltage for normal station configuration and nominal system parameters. 


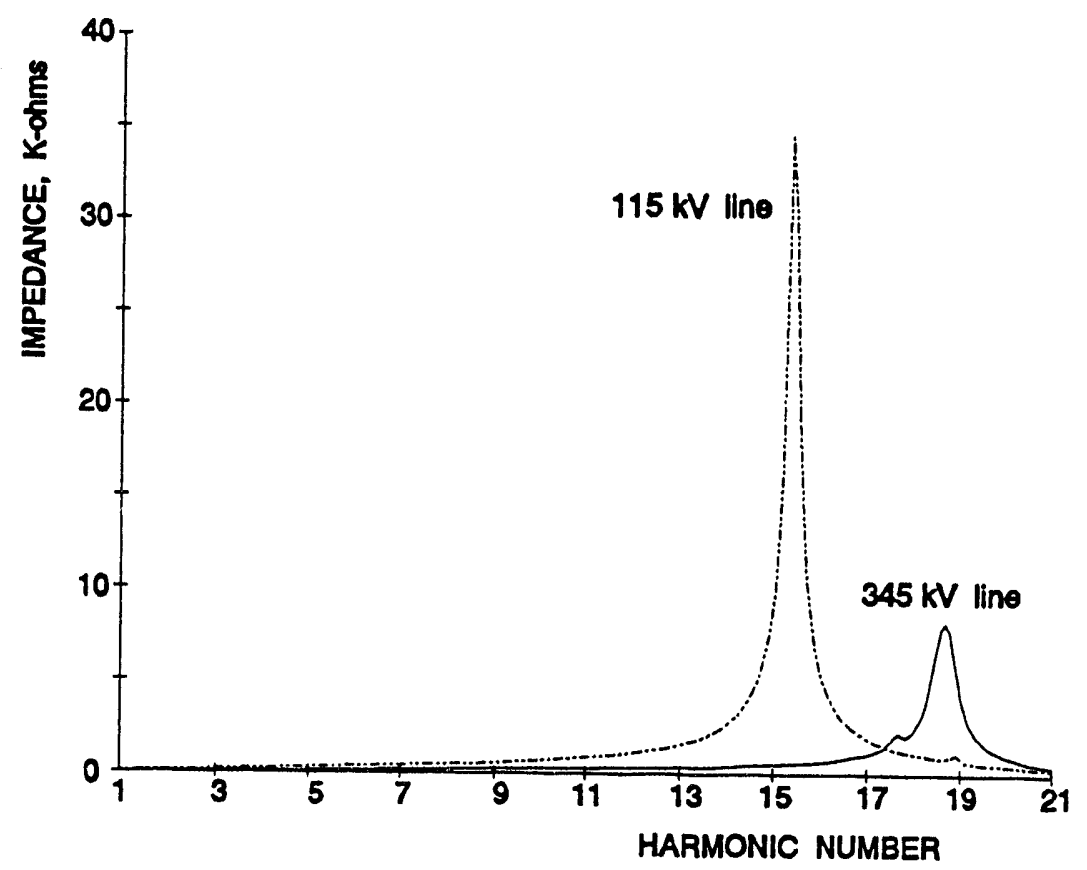

Fig. G-5. Transmission line impedance functions for station with separate $115 \mathrm{kV}$ service and nominal system parameters.

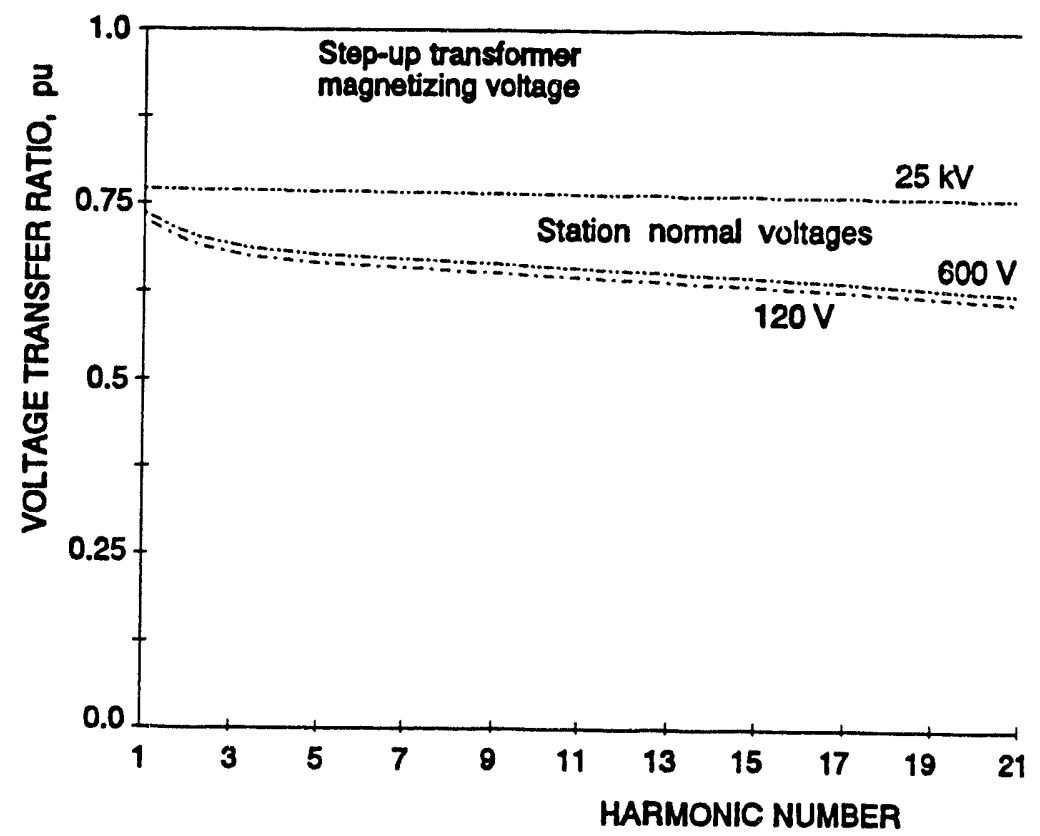

Fig. 6-6. Transfer ratio of nonsafety-related distribution voltages to transformer excitation voltage for station with separate $115 \mathrm{kV}$ service and nominal system parameters. 


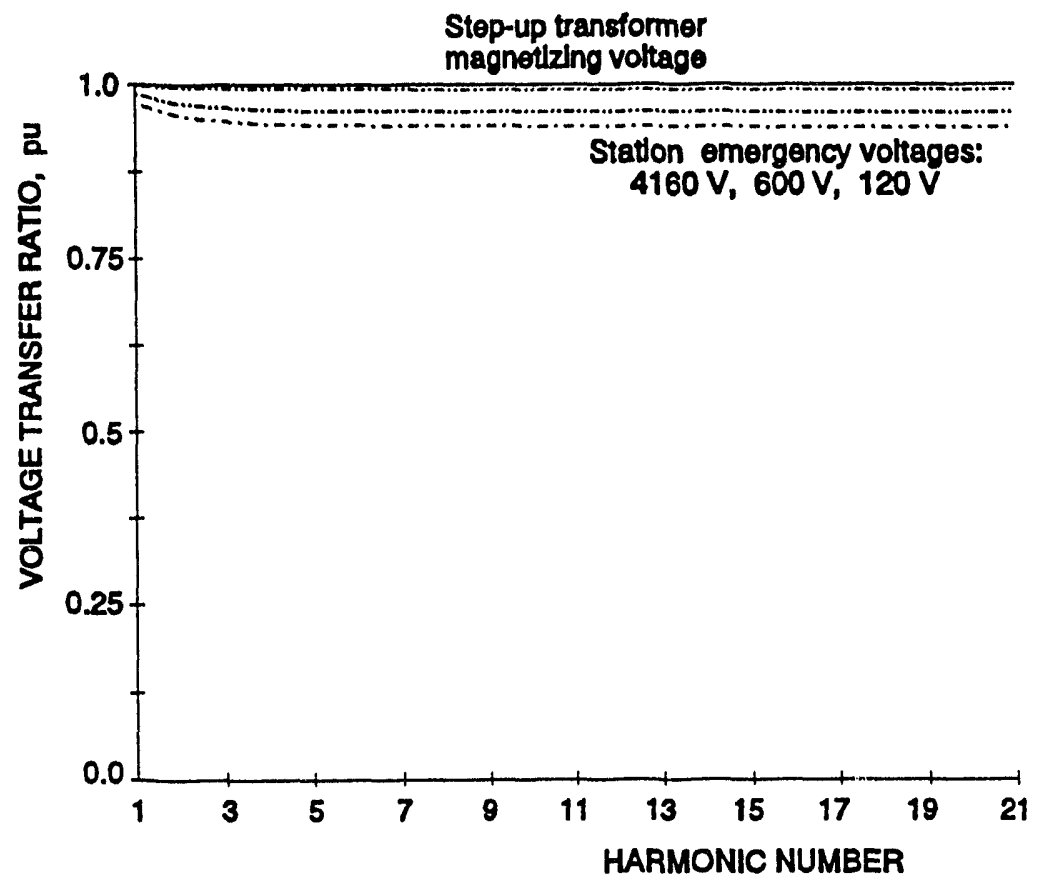

Fig. G-7. Transfer ratio of safety-related distribution voltages to transformer excitation voltage for station with separate 115 $\mathrm{kV}$ service and nominal system parameters.

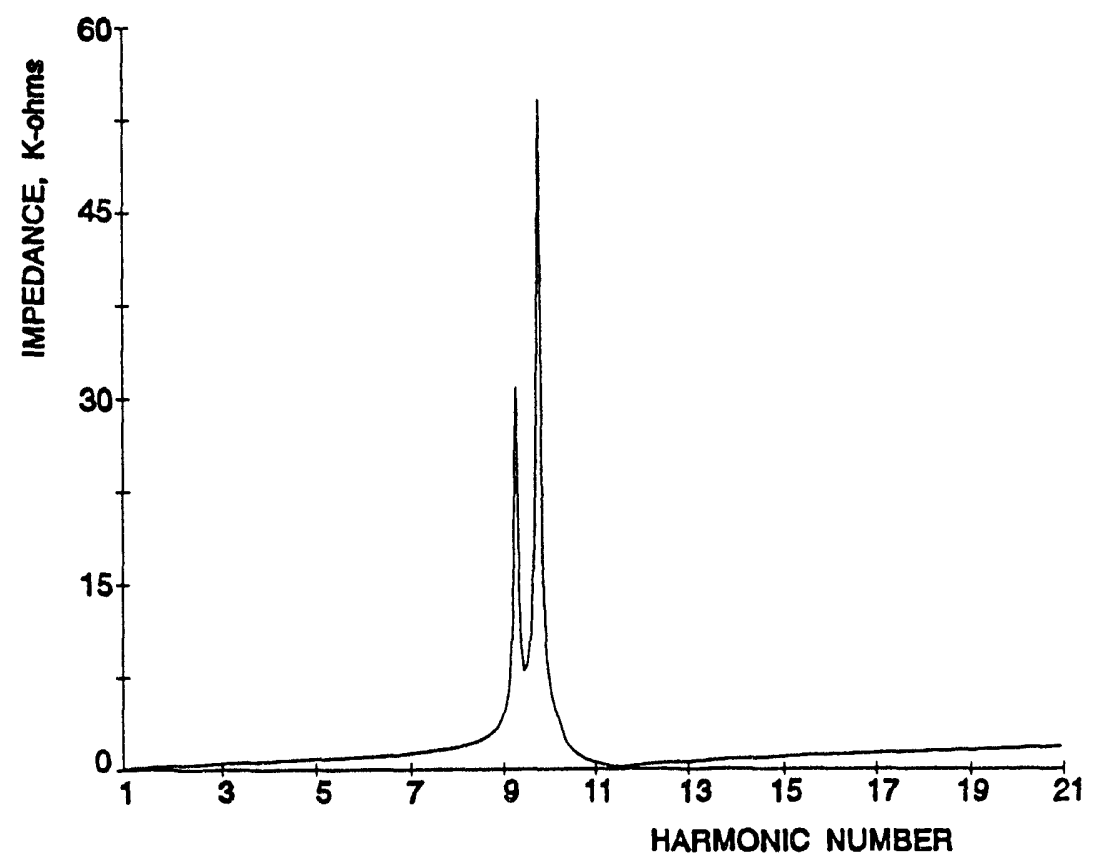

Fig. 6-8. Transmission 1 ine impedance function for station startup mode with nominal system parameters. 


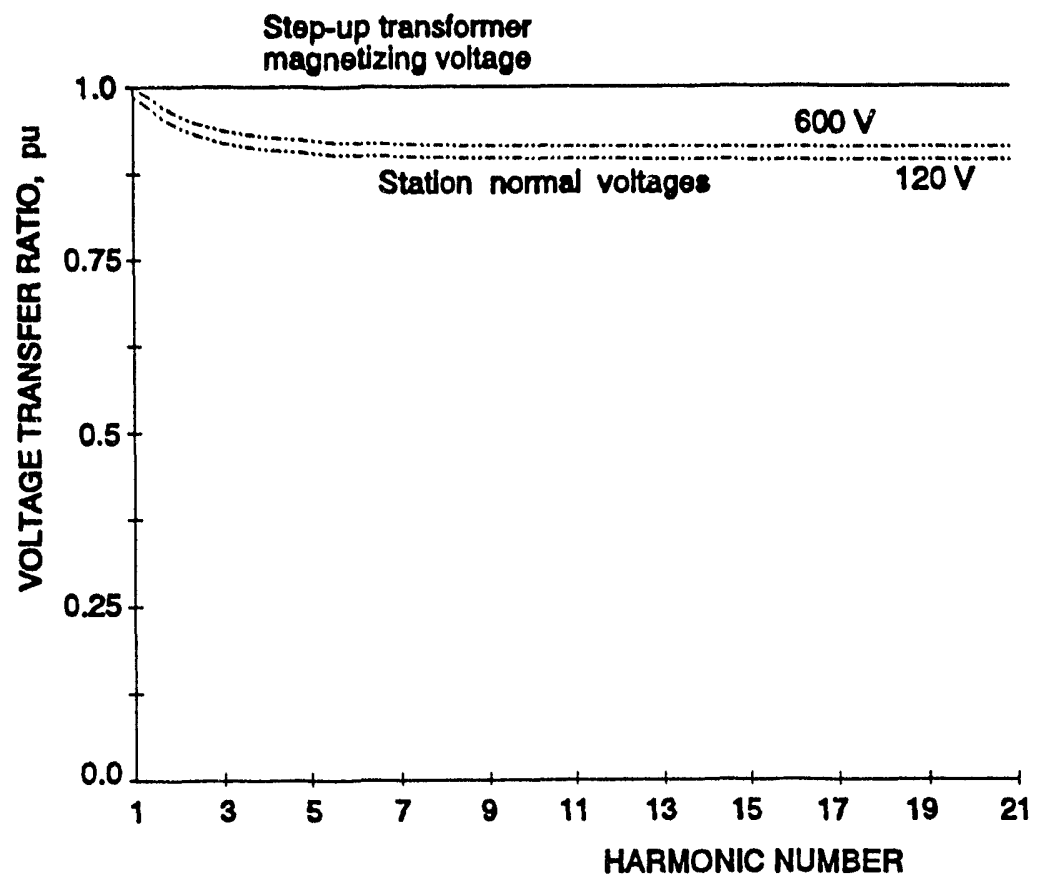

Fig. G-9. Transfer ratio of nonsafety-related distribution voltages to transformer excitation voltage for station start-up mode with nominal system parameters.

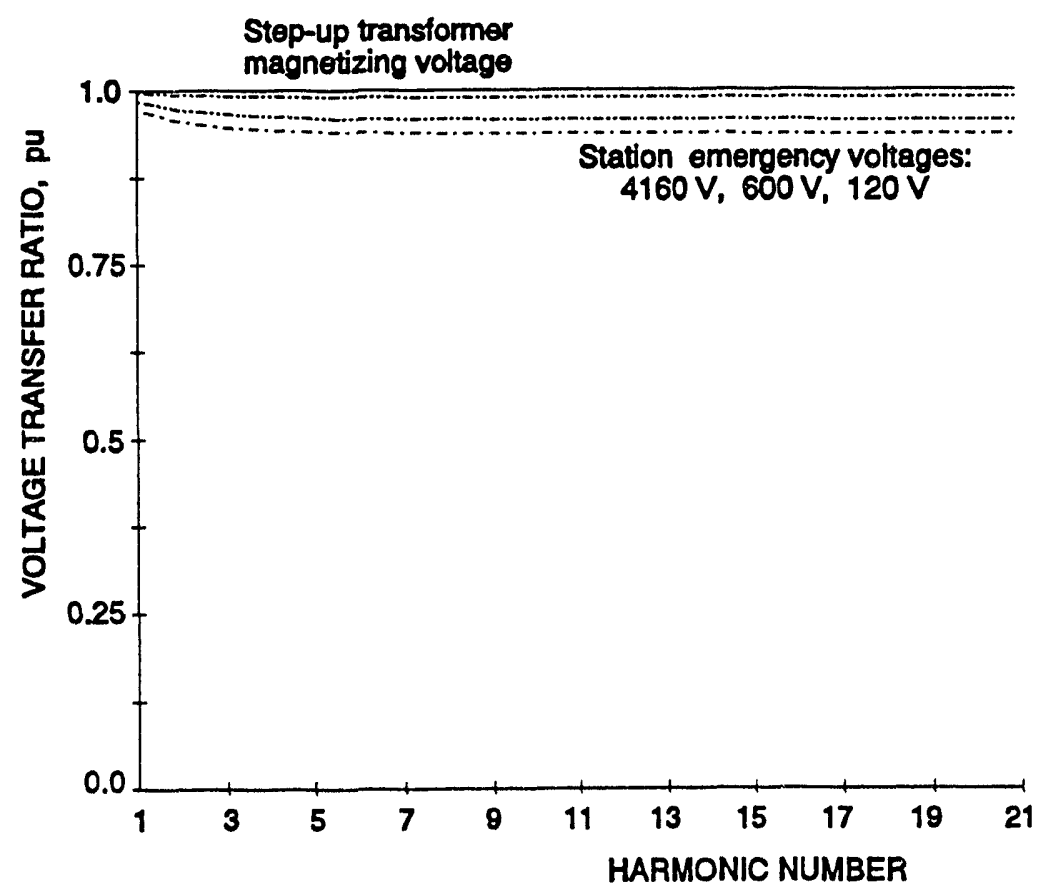

Fig. G-10. Transfer ratio of safety-related distribution voltages to transformer excitation voltage for station start-up mode with nominal system parameters. 


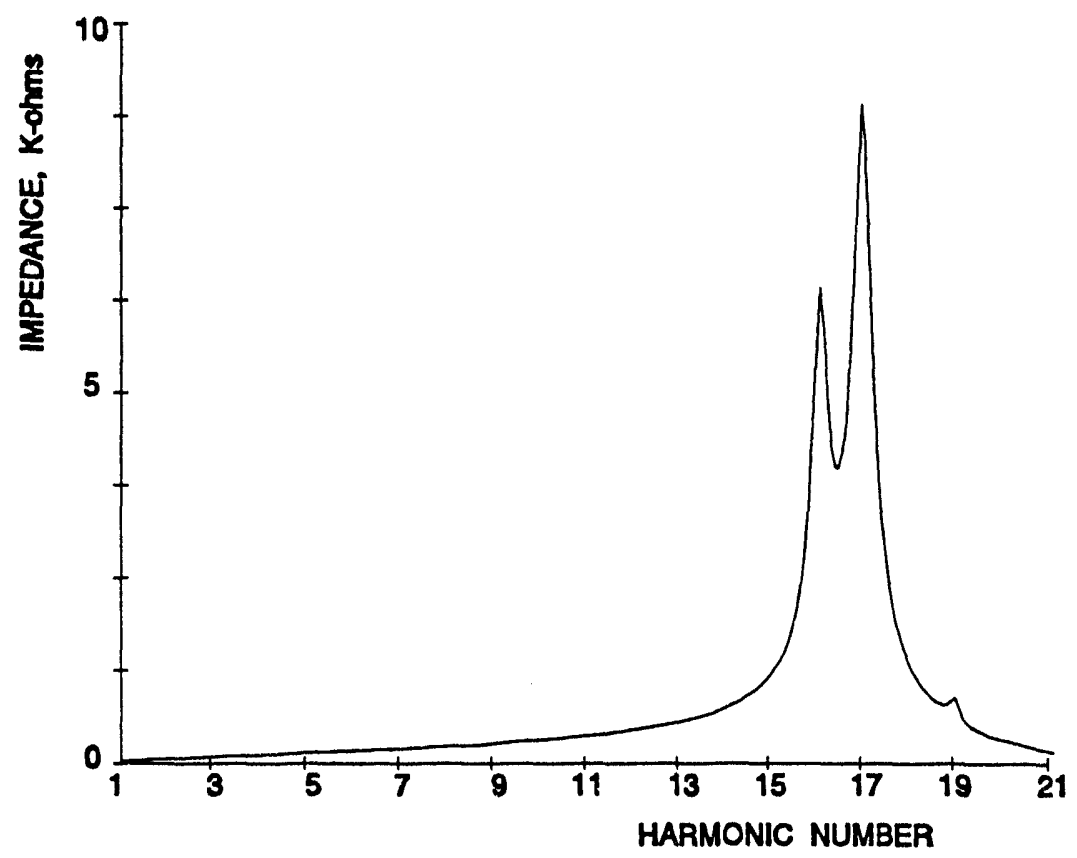

Fig. G-11. Transmission line impedance function for normal station configuration with 42.0 mile line and remote source impedance.

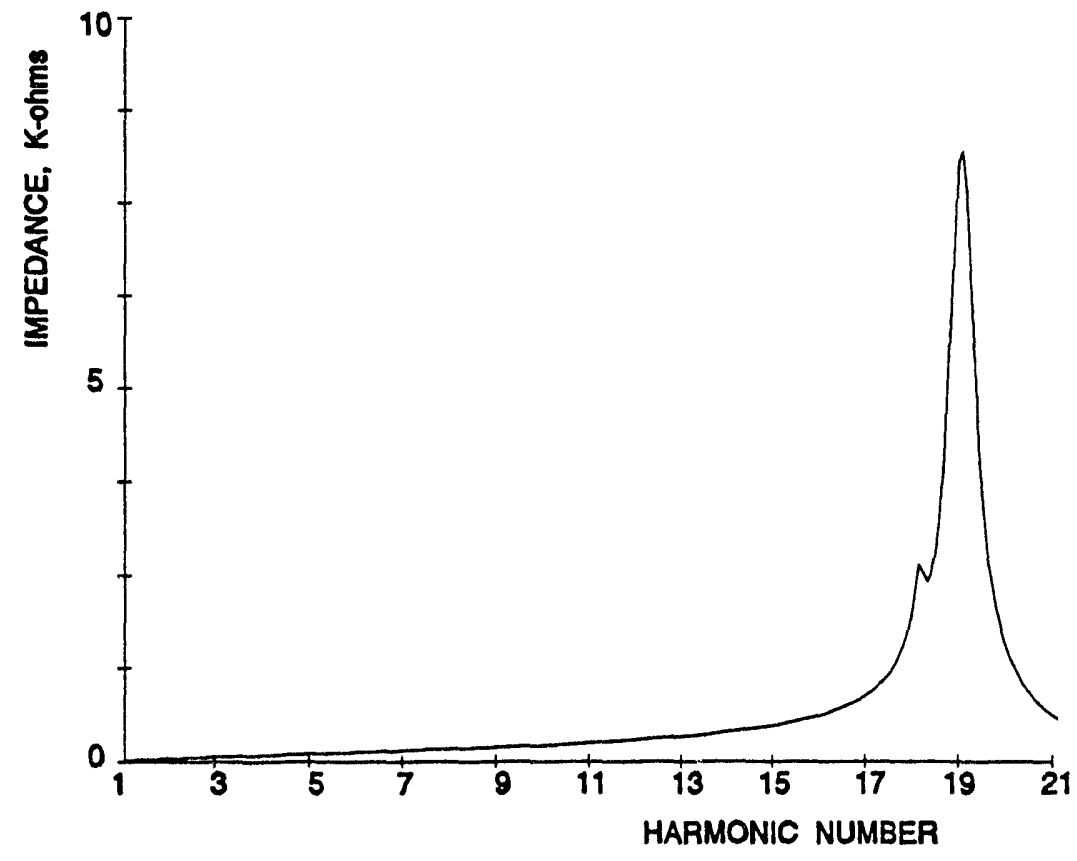

Fig. G-12. Transmission line impedance function for normal station configuration with 49.07 mile line.

$$
G-6
$$




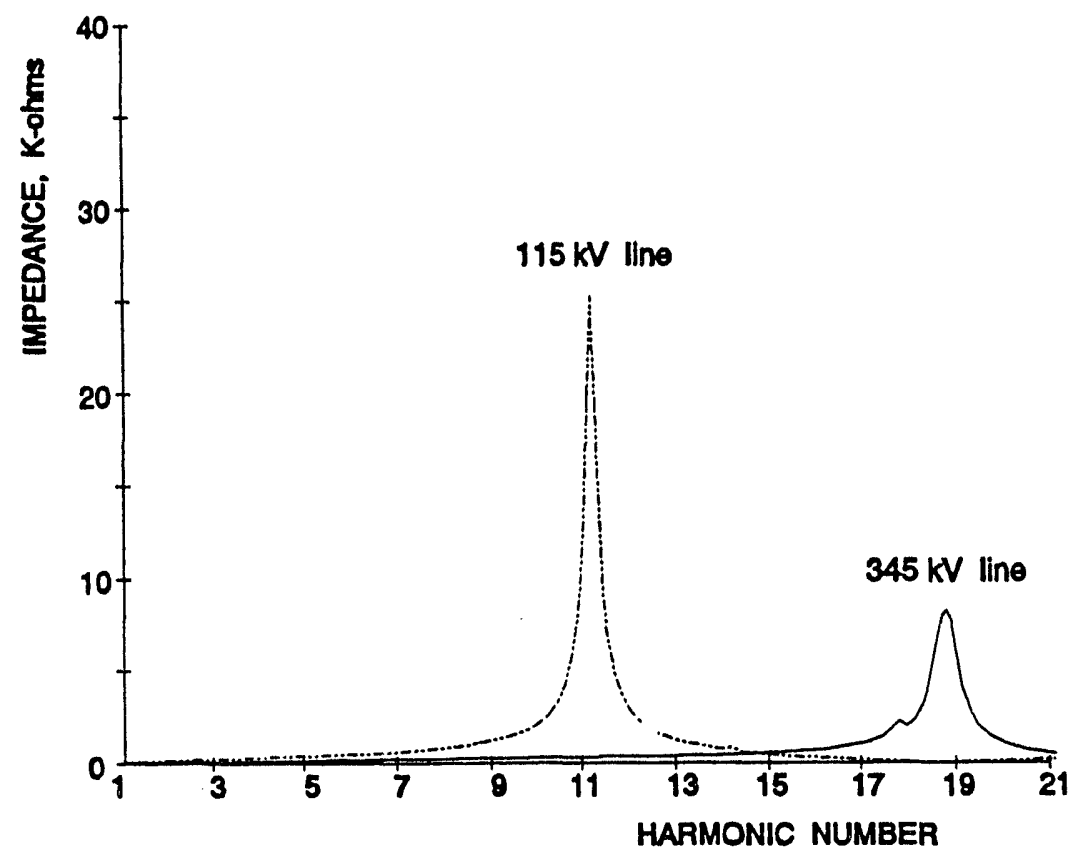

Fig. G-13. Transmission line impedance functions for station with separate $115 \mathrm{kV}$ service $(69.55 \mathrm{mi}$ line).

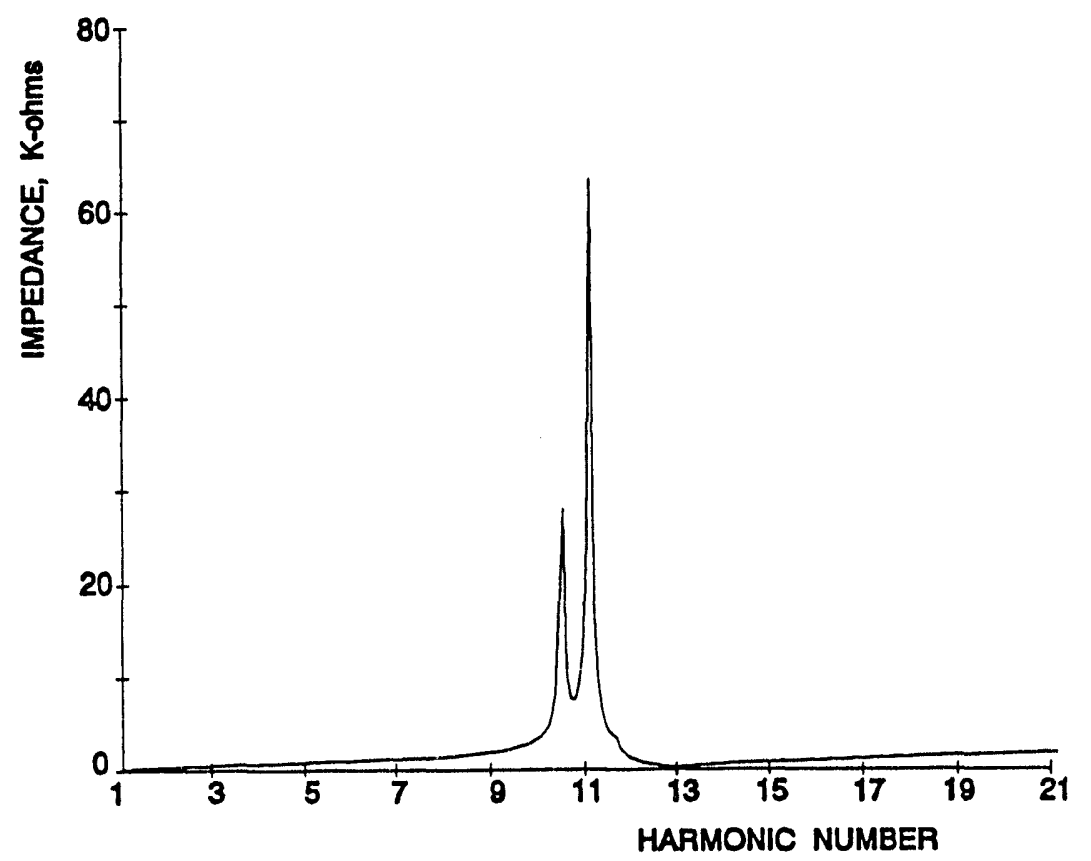

Fig. G-14. Transmission line impedance function for station start-up mode with $42.38 \mathrm{ml}$ line. 


\section{APPENDIX H: Extended Simulation Studies}

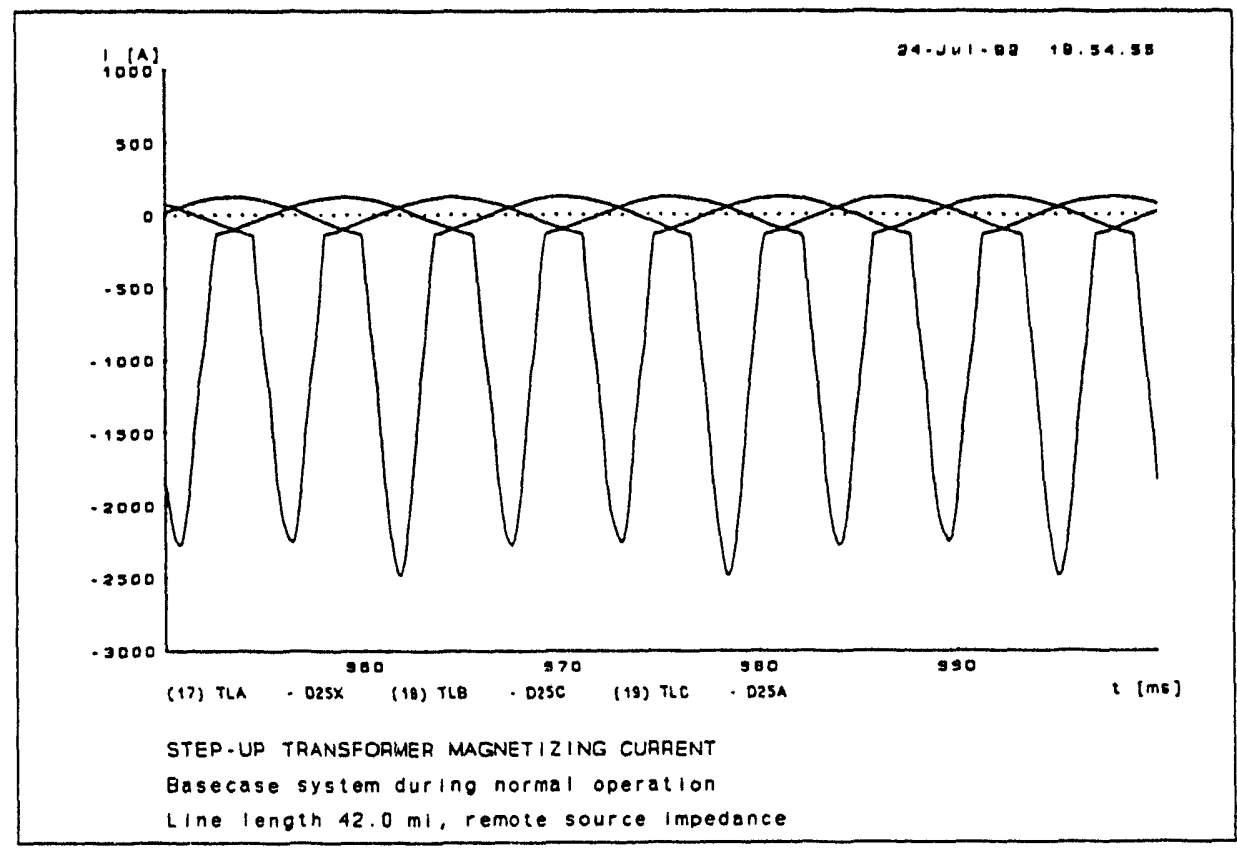

Fig. B-1. Step-up transformer magnetizing currents during normal operation (100 A GIC) with $42.0 \mathrm{mi}$ line and remote source impedance.

FREQUENCY SPECTRUM

Step-up Transformer Magnetizing Current

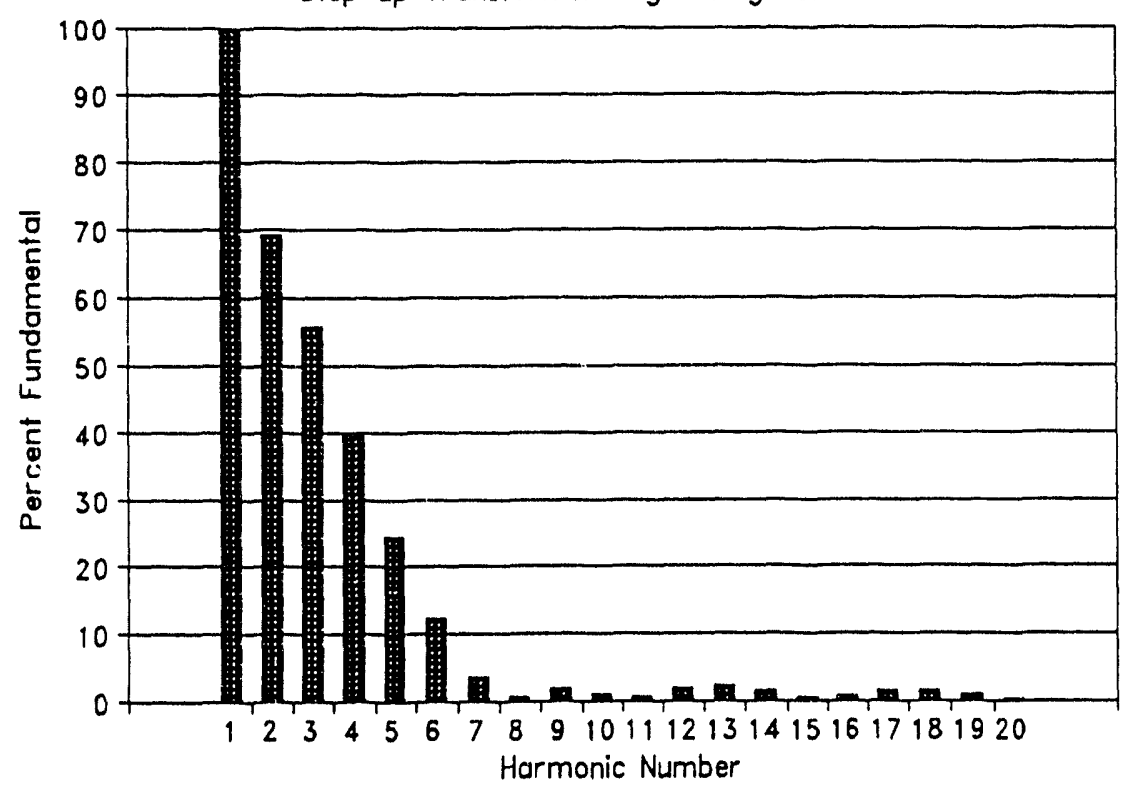

Fig. B-2. Frequency spectrum of generator step-up transformer magnetizing current.

$$
\mathrm{H}-1
$$




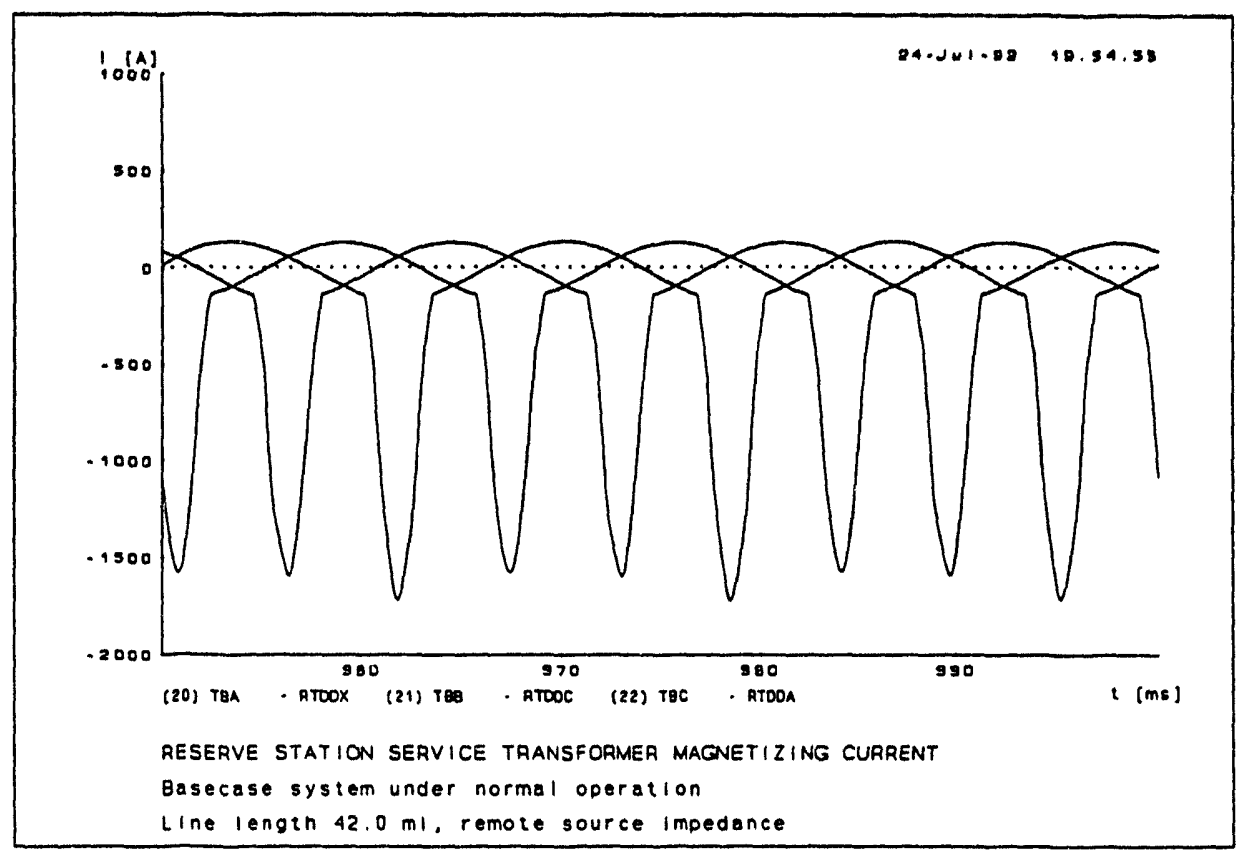

Fig. H-3. Reserve station service trangformer magnetizing currents during normal operation (100 A GIC) with $42.0 \mathrm{mi}$ line and remote source impedance.

FREQUENCY SPECTRUM

Reserve Transformer Magnetizing Current

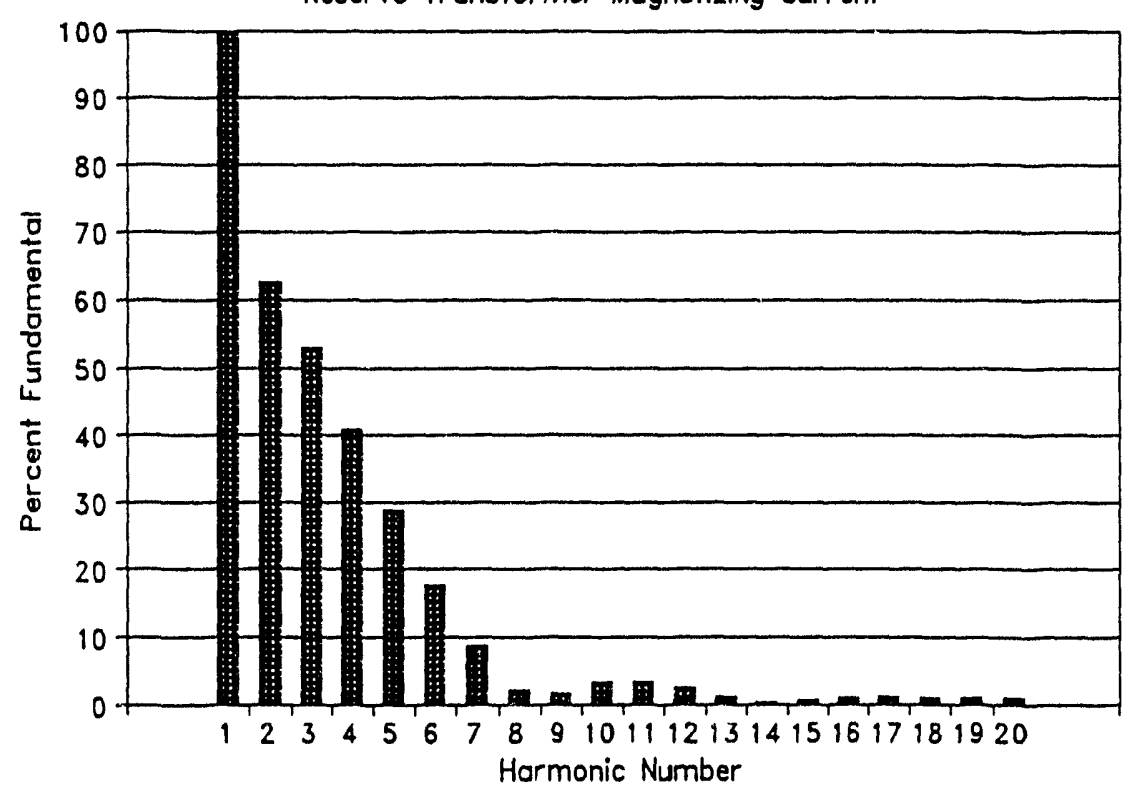

Fig. B-4. Frequency spectrum of reserve station service transformer magnetizing current. 


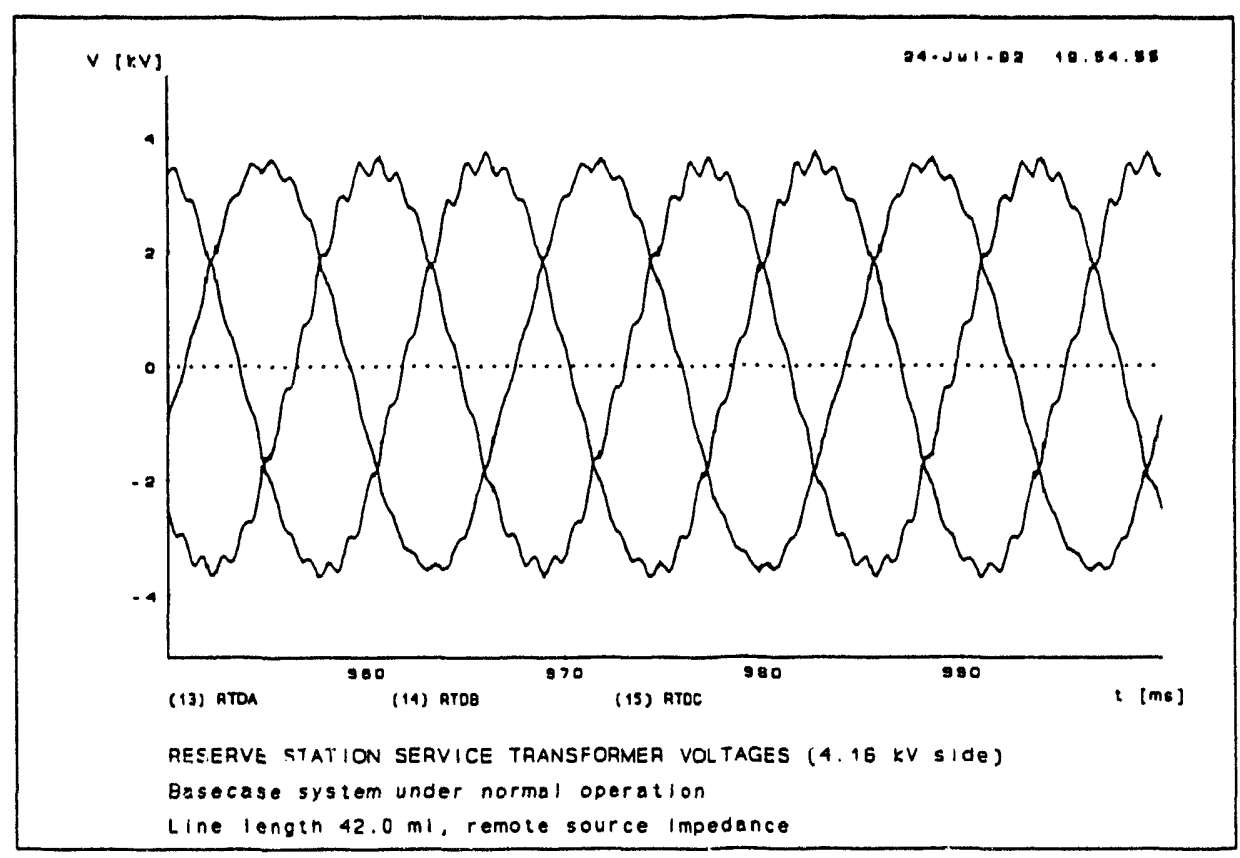

Fig. H-5. Reserve station service transformer voltages on $4.16 \mathrm{kV}$ side during normal operation (100 A GIC) with $42.0 \mathrm{mi}$ line and remote source impedance.

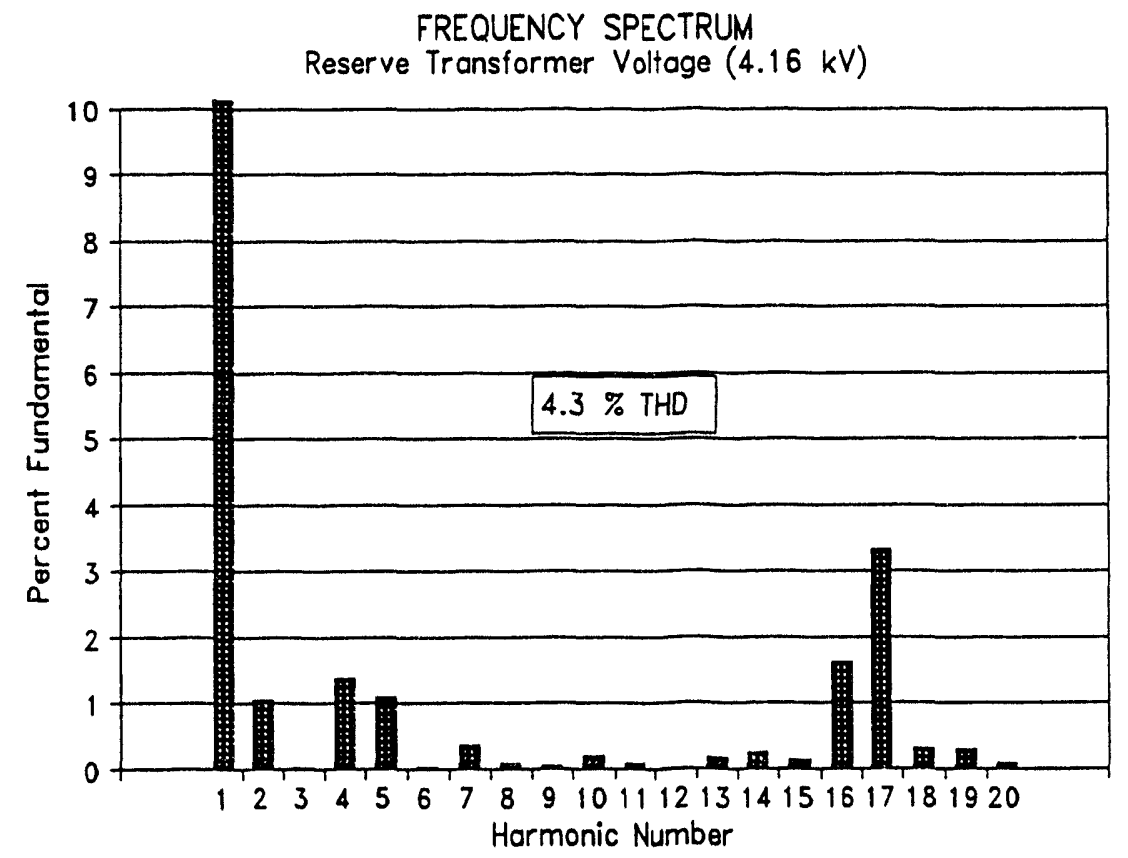

Fig. B-6. Frequency spectrum of phase A reserve station service transformer voltage on LV side $(4.16 \mathrm{kV})$. 


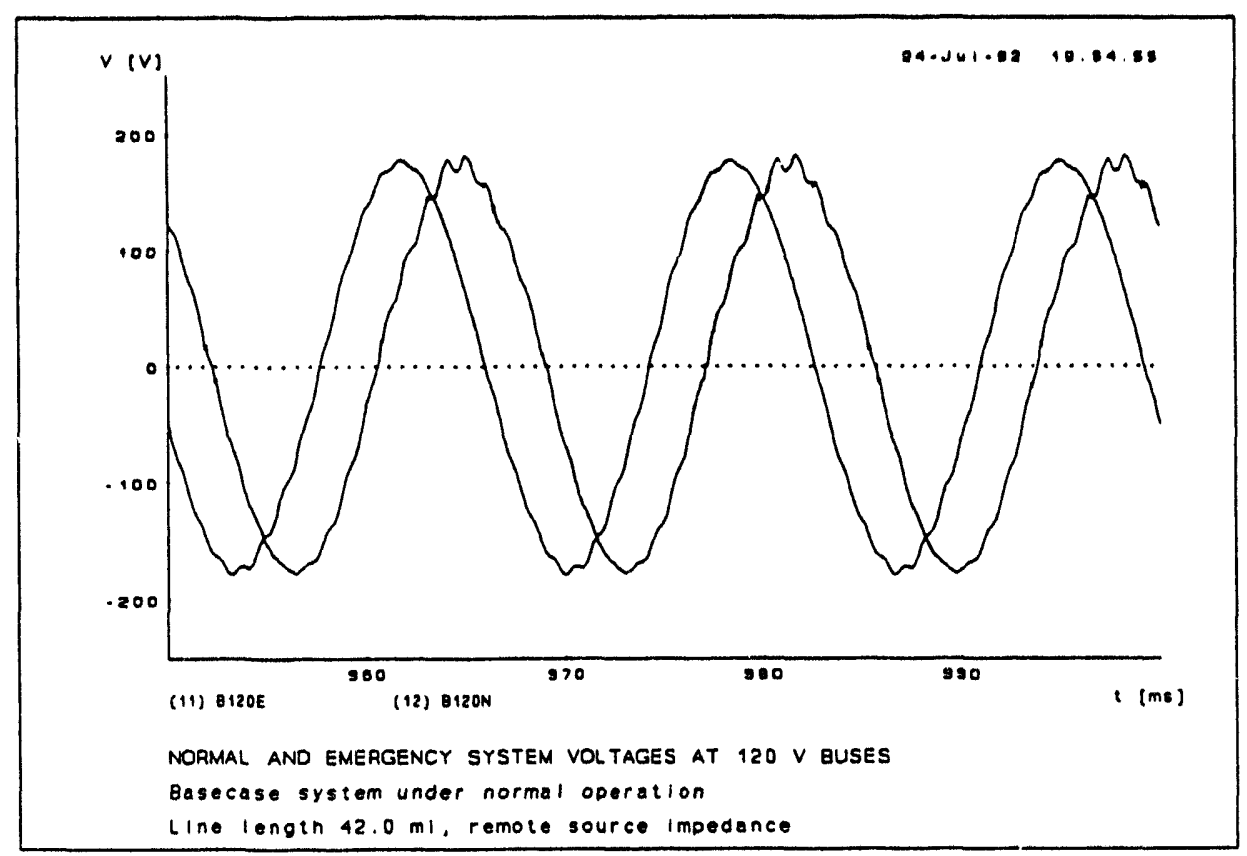

Fig. H-7. Normal and emergency voltages at $120 \mathrm{~V}$ buses during normal operation ( 100 A GIC) with $42.0 \mathrm{mi}$ line and remote source impedance.

FREQUENCY SPECTRUM

$120 \mathrm{~V}$ Emergency System Voltage

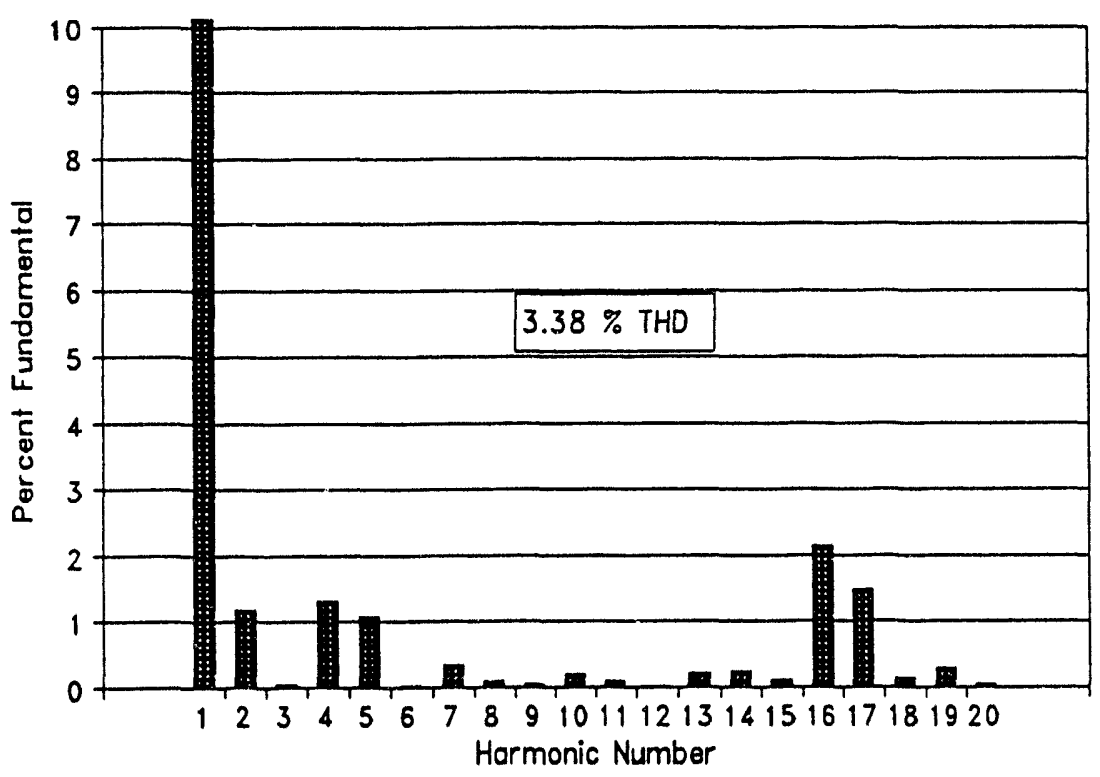

Fig. $8-8$. Frequency spectrum of $120 \mathrm{~V}$ emergeney system voltage. 


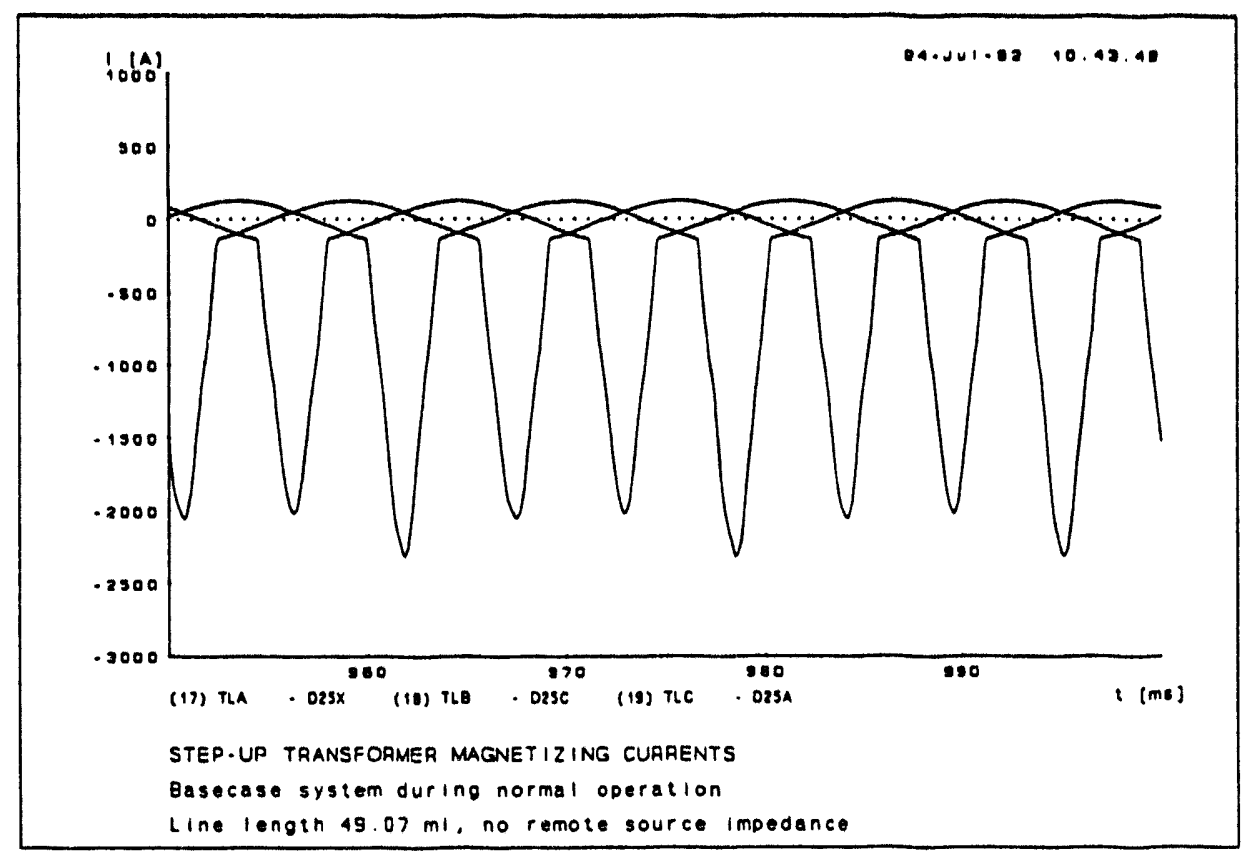

Fig. H-9. Step-up transformer magnetizing currents during normal operation (100 A GIC) with $49.07 \mathrm{mi}$ line.

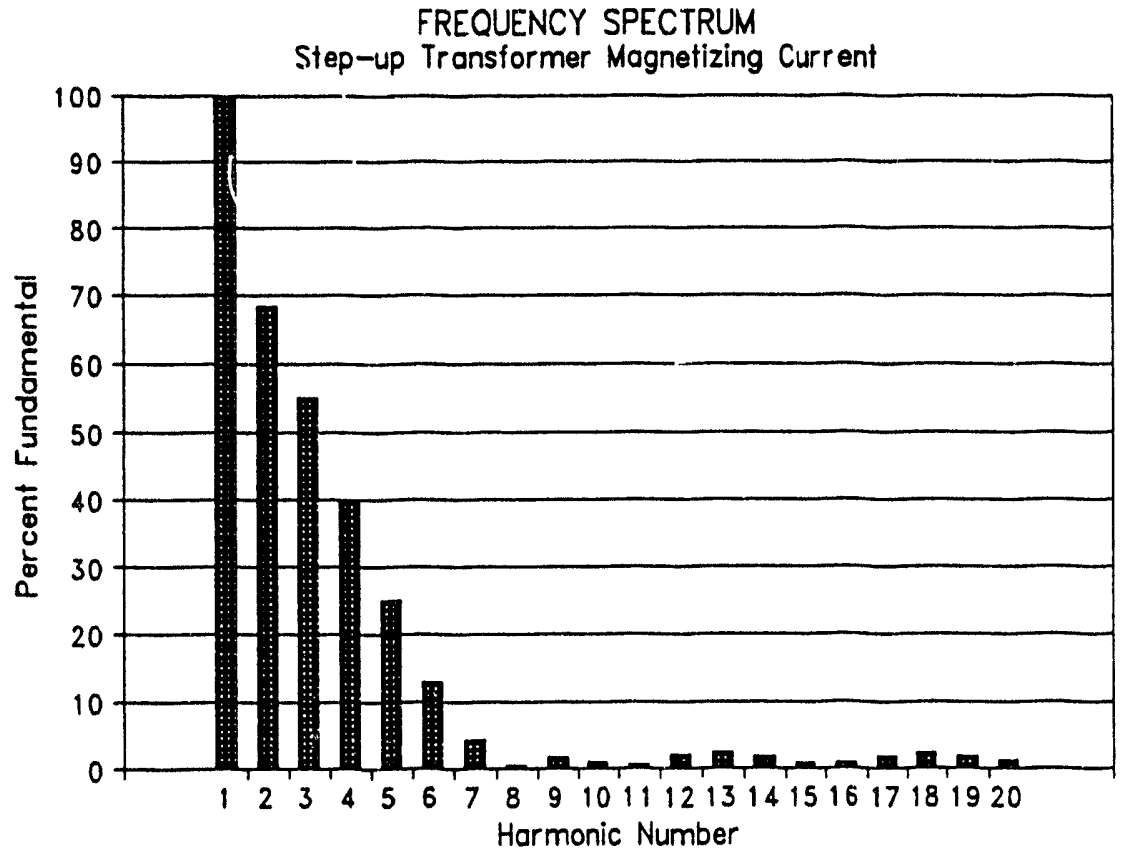

Fig. H-10. Frequency spectrum of generator step-up transformer magnetizing current. 


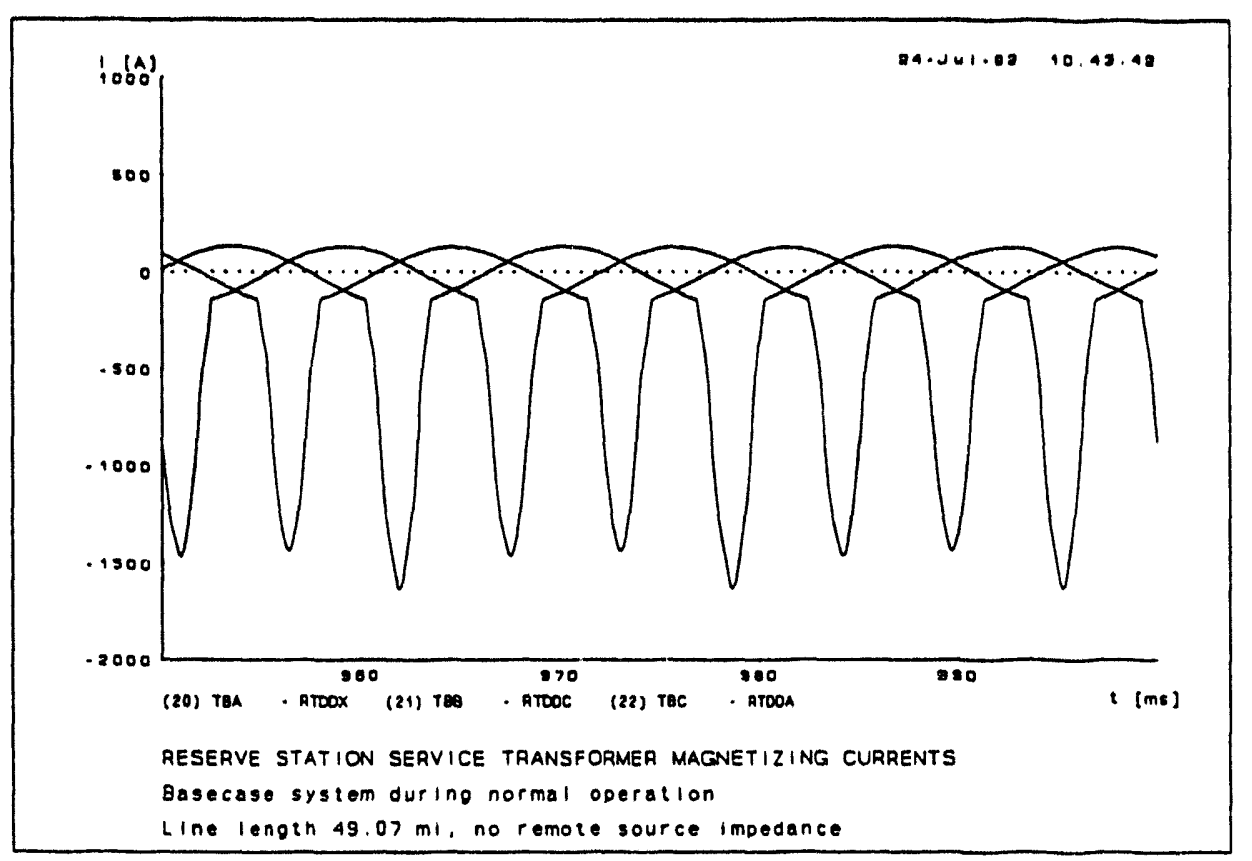

Fig. H-11. Reserve station service transformer magnetizing currents during normal operation (100 A GIC) with $49.07 \mathrm{mi}$ line.

FREQUENCY SPECTRUM

Reserve Transformer Magnetizing Current

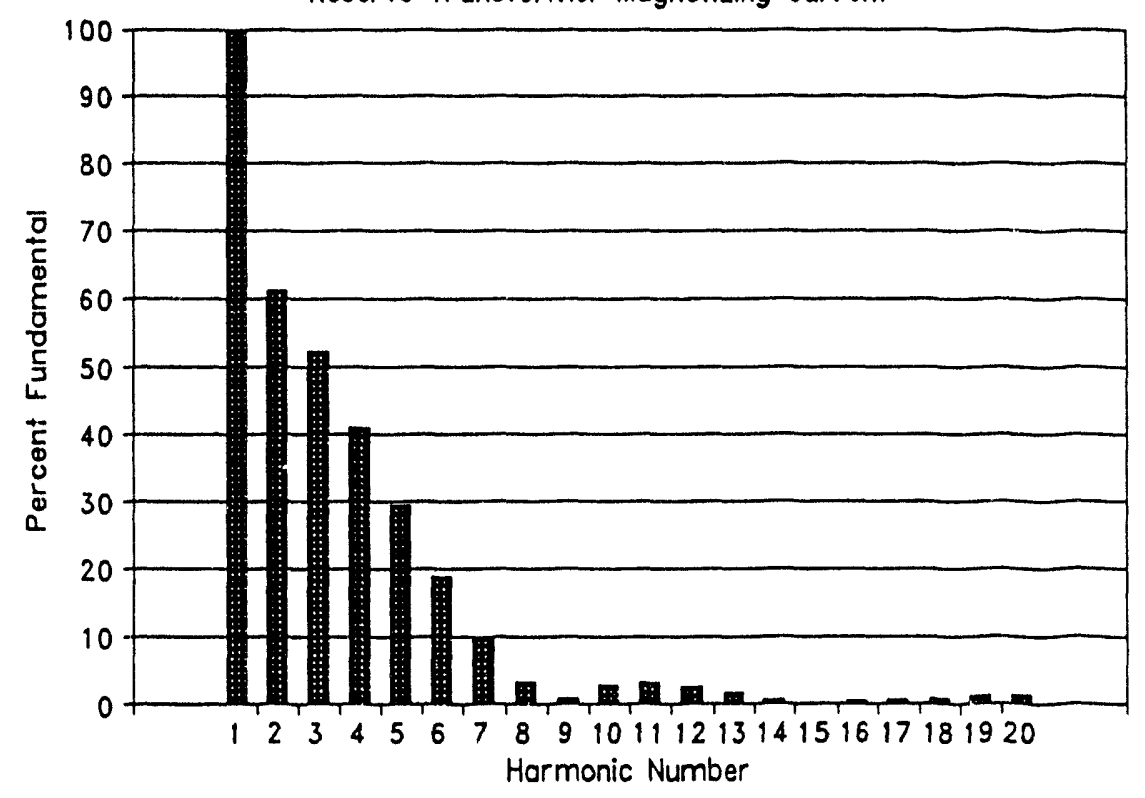

Fig. B-12. Frequency spectrum of reserve station service transformer magnetizing current. 


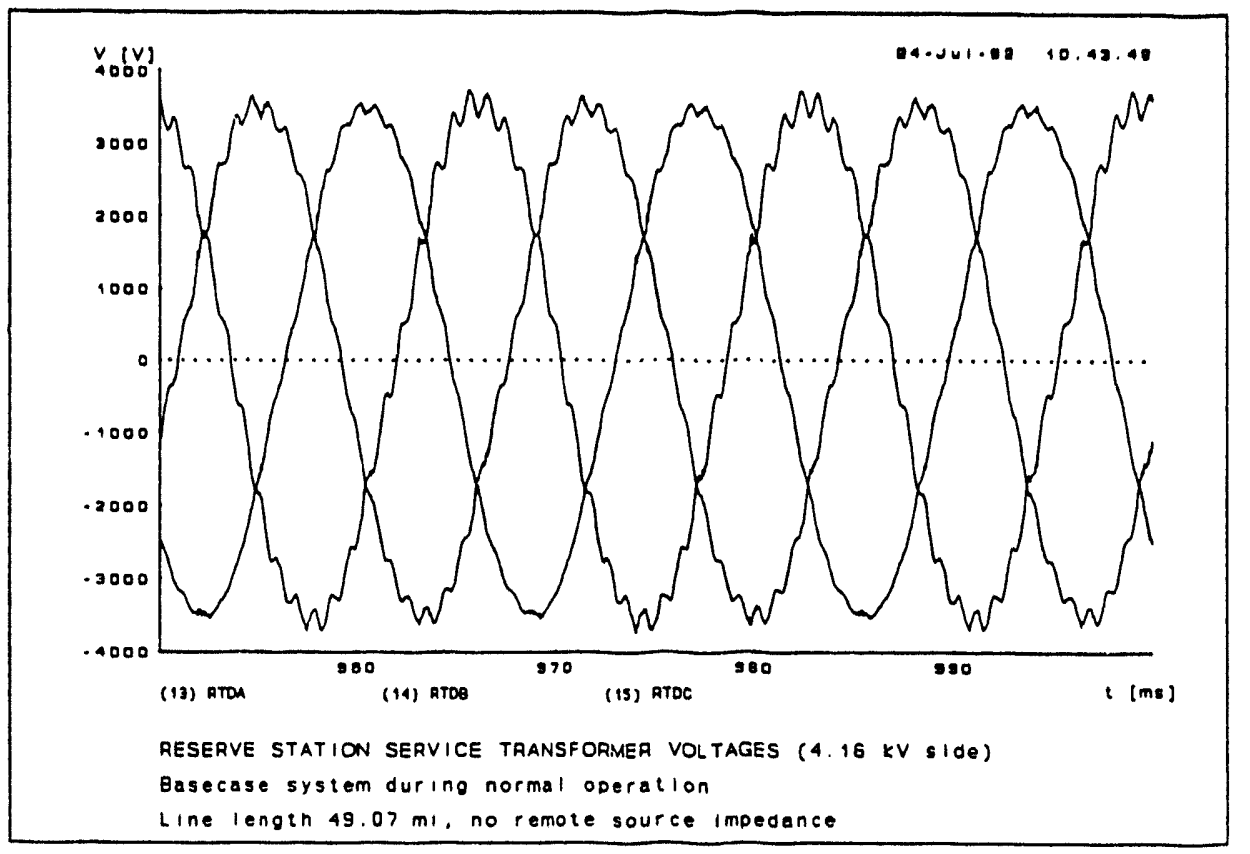

Fig. H-13. Reserve station service transformer voltages on 4.16 $\mathrm{kV}$ side during normal operation (100 A GIC) with $49.07 \mathrm{mi}$ line.

FREQUENCY SPECTRUM

Reserve Transformer Voltage $(4.16 \mathrm{kV})$

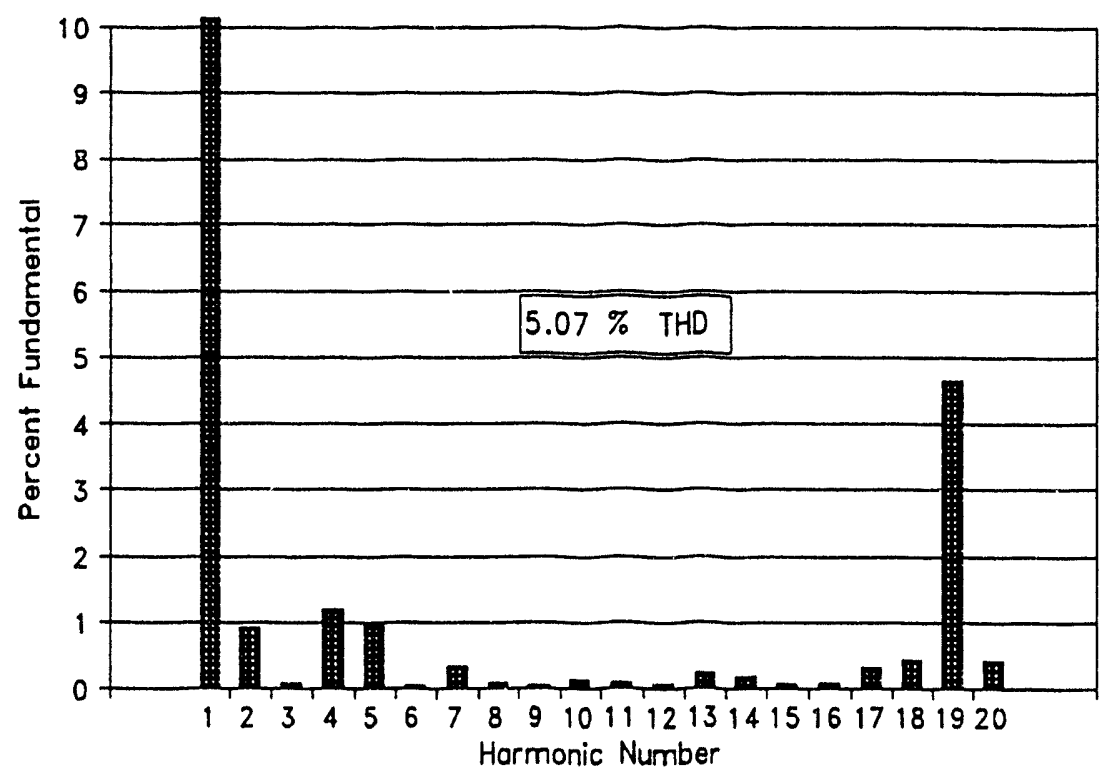

Fig. B-14. Frequency spectrum of phase A reserve station service transformer voltage on LV side $(4.16 \mathrm{kV})$. 


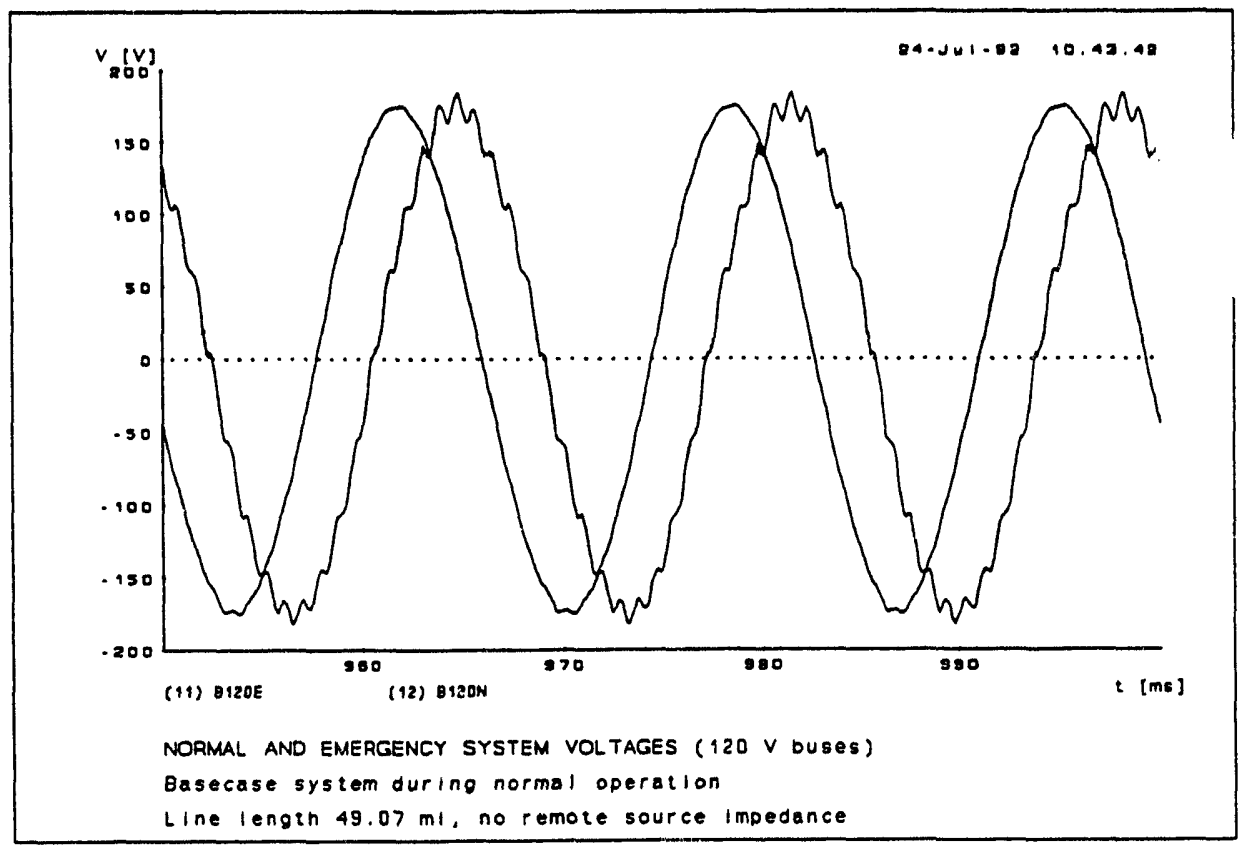

Fig. H-15. Normal and emergency system voltages at $120 \mathrm{~V}$ buses during normal operation (100 A GIC) with $49.07 \mathrm{mi}$ line.

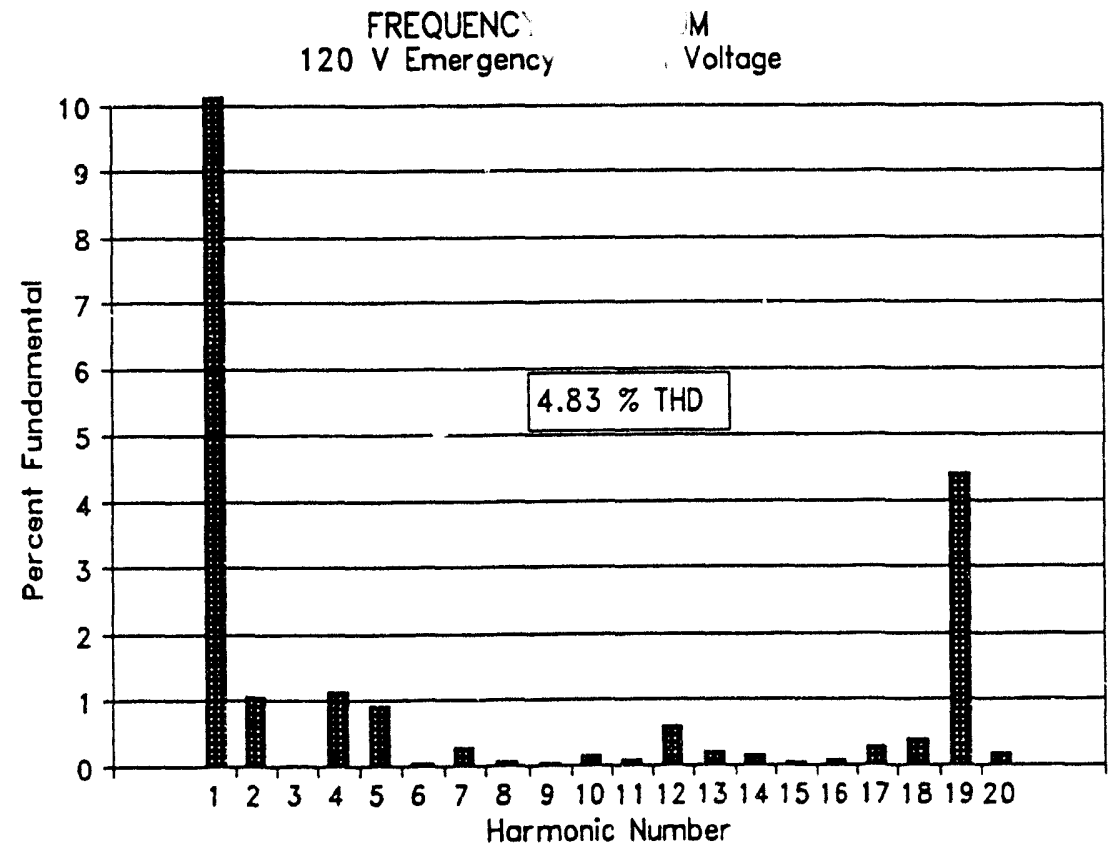

Fig. B-16. Frequency spectrum of $120 \mathrm{~V}$ emergency system voltages. 


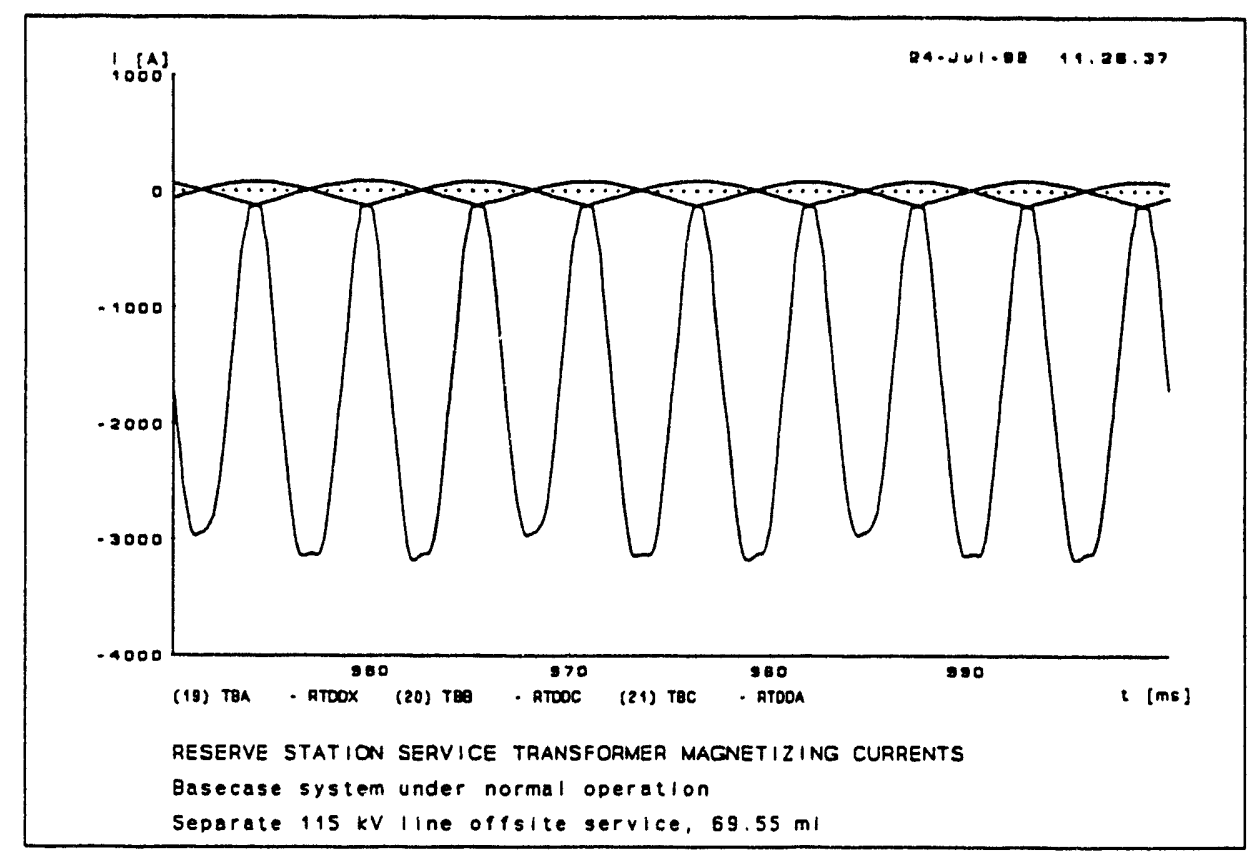

Fig. I-17. Reserve station service transformer magnetizing currents during normal operation (100 A GIC) with separate 69.55 mi $115 \mathrm{kV}$ line.

FFEQUENCY SPECTRUM

Reserve Transformer Magnetizing Current

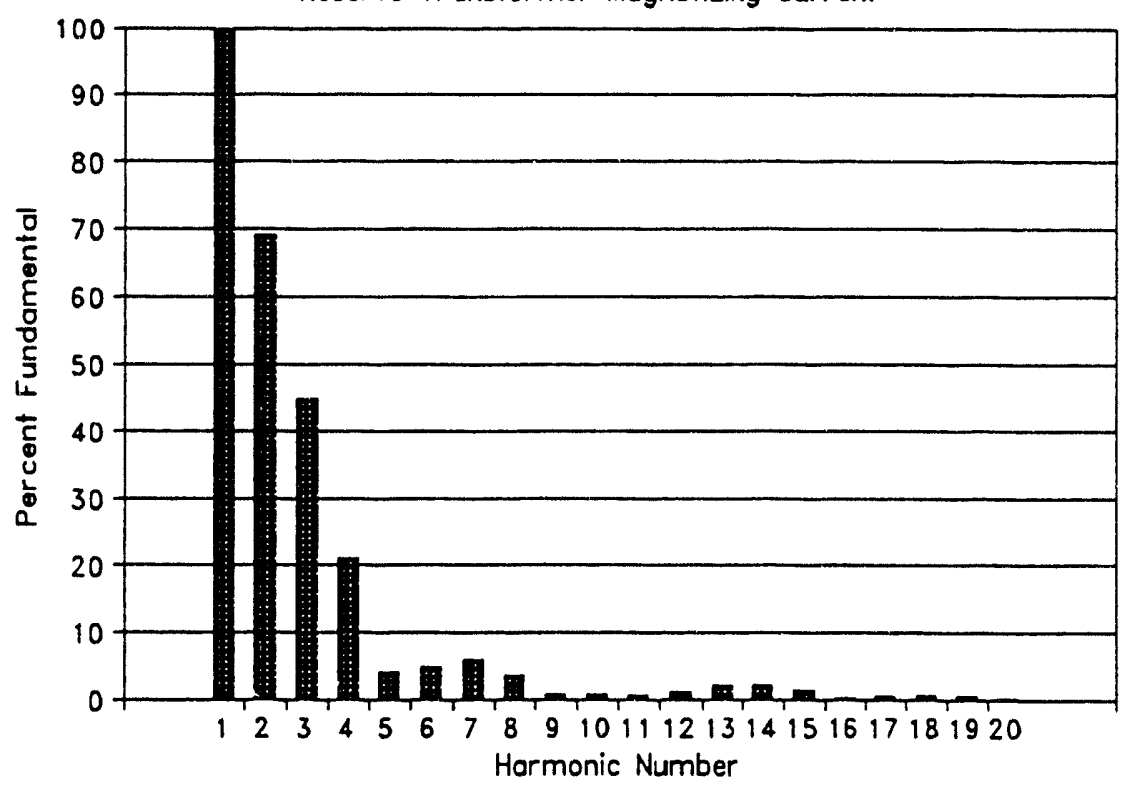

Fig. E-18. Frequency spectrum of reserve station service transformer magnetizing current. 


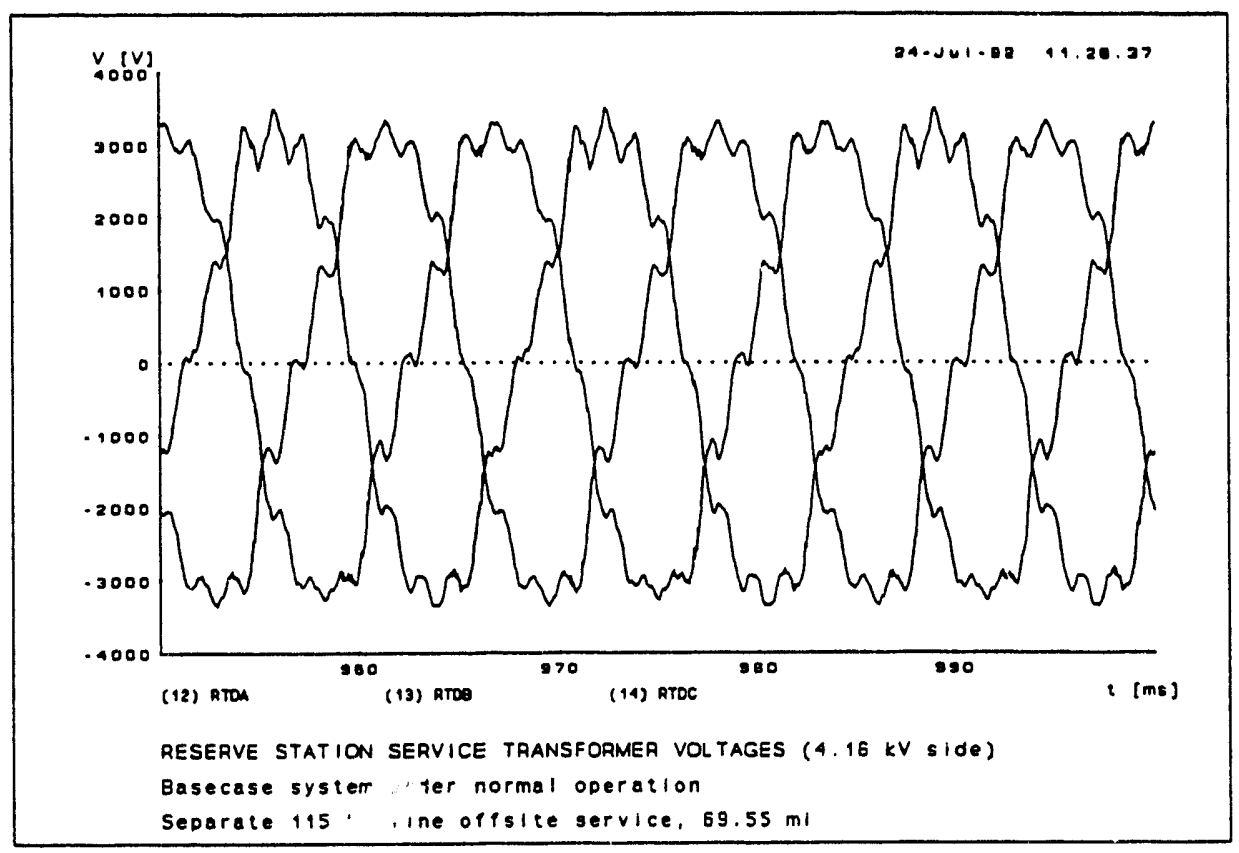

Fig. H-19. Reserve station service transformer voltages on 4.16 $\mathrm{kV}$ side during normal operation (100 A GIC) with separate 69.55 mi $115 \mathrm{kV}$ line.

FREOUENCY SPECTRUM

Reserve Transformer Voltage $(4.16 \mathrm{kV})$

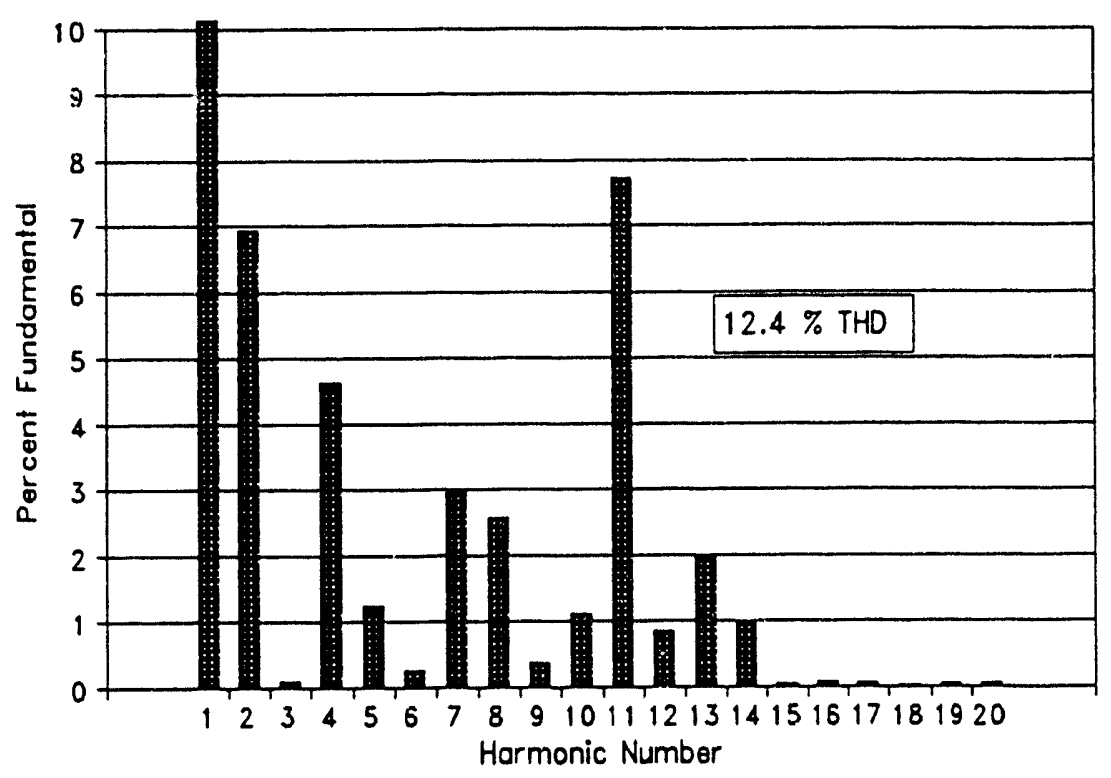

Fig. B-20. Frequency spectrum of phase A reserve station service transformer voltage on IV side $(4.16 \mathrm{kV})$. 


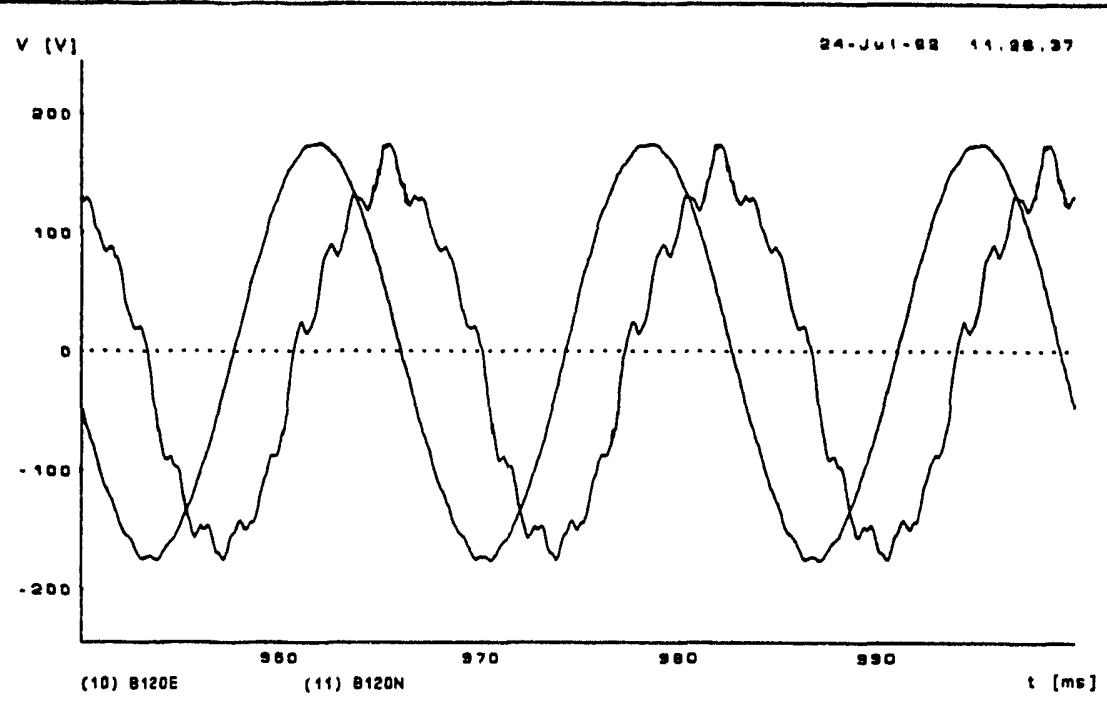

NORMAL AND EMERGENCY SYSTEM VOLTAGES ( $120 \mathrm{~V}$ buses)

Basecase system under normal operation

Separate $115 \mathrm{kV}$ line offsite service, $69.55 \mathrm{ml}$

Fig. H-21. Normal and emergency system voltages at $120 \mathrm{~V}$ buses during normal operation ( $100 \mathrm{~A}$ GIC) with separate $69.55 \mathrm{mi} 115 \mathrm{kV}$ line.

FREQUENCY SPECTRUM

$120 \mathrm{~V}$ Emergency System Voltage

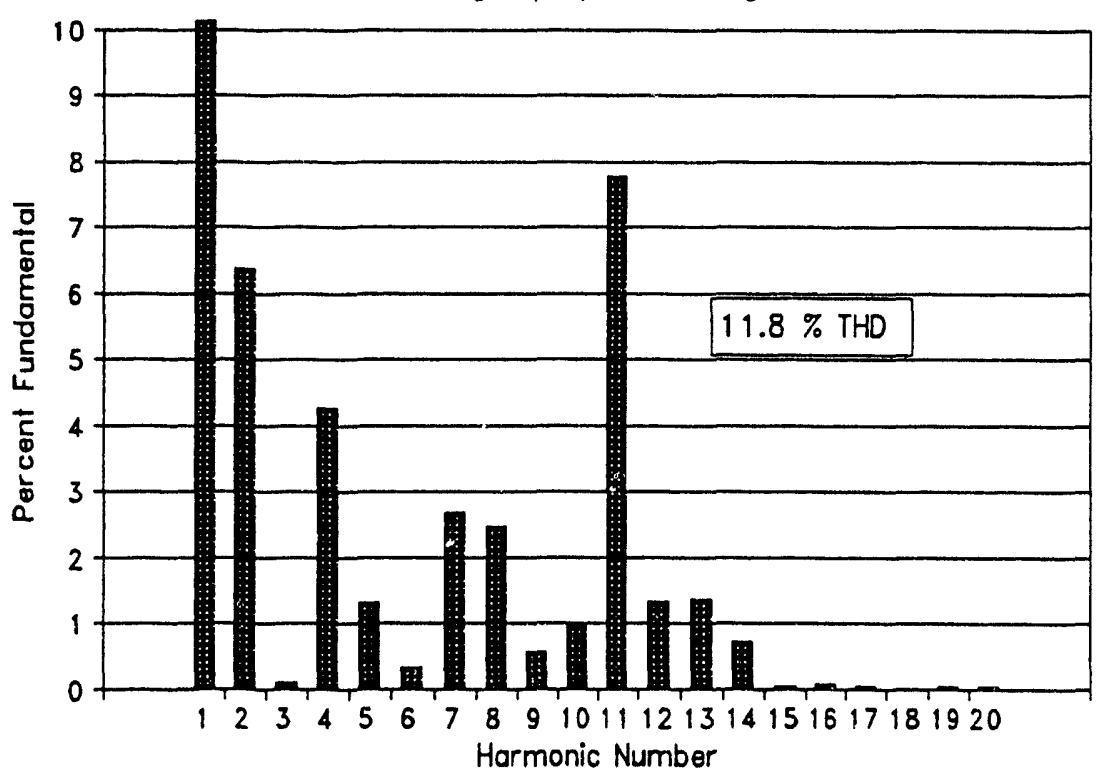

Fig. B-22. Frequency spectrum of $120 \mathrm{~V}$ emergency system voltage. 


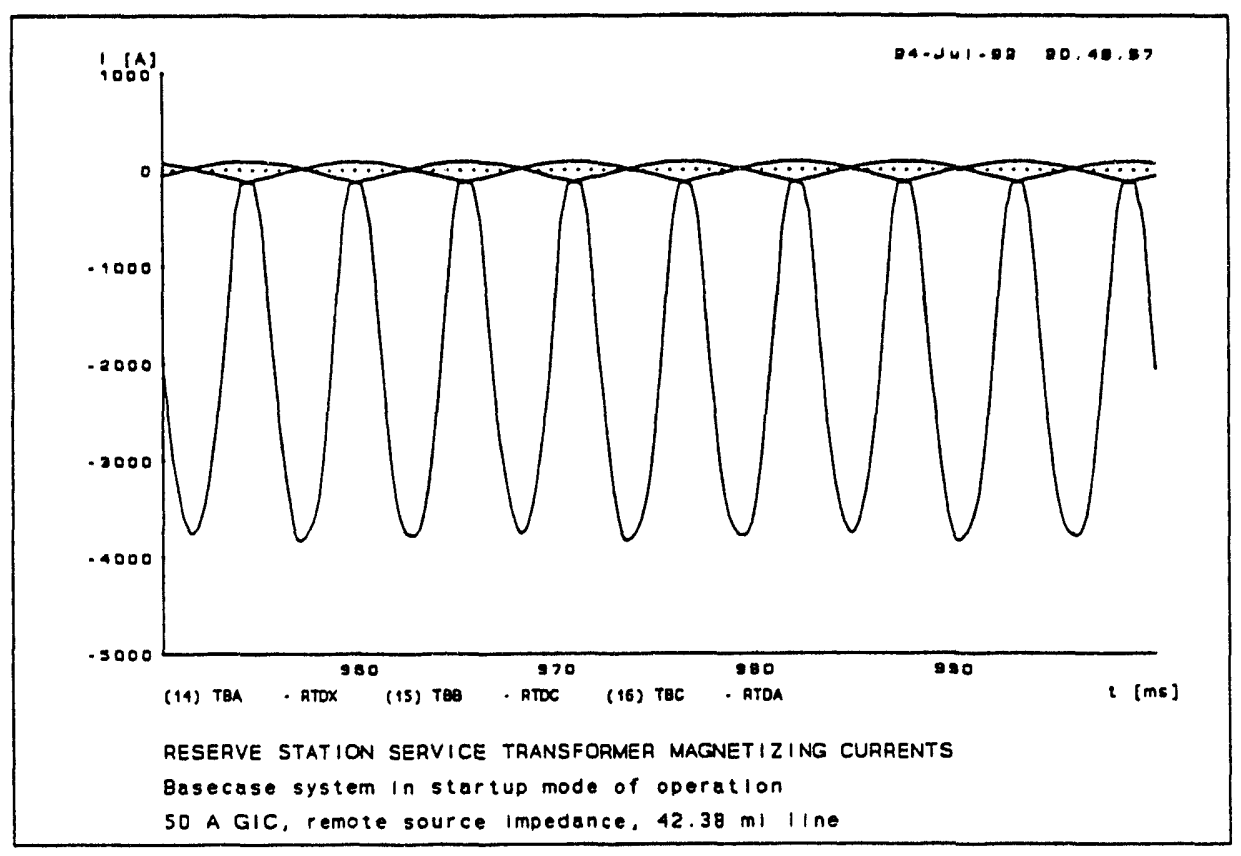

Fig. B-23. Reserve station service transformer magnetizing currents during start-up mode (50 A GIC) with $42.38 \mathrm{mi}$ line and remote source impedance.

FREQUENCY SPECTRUM

Reserve Transformer Magnetizing Current

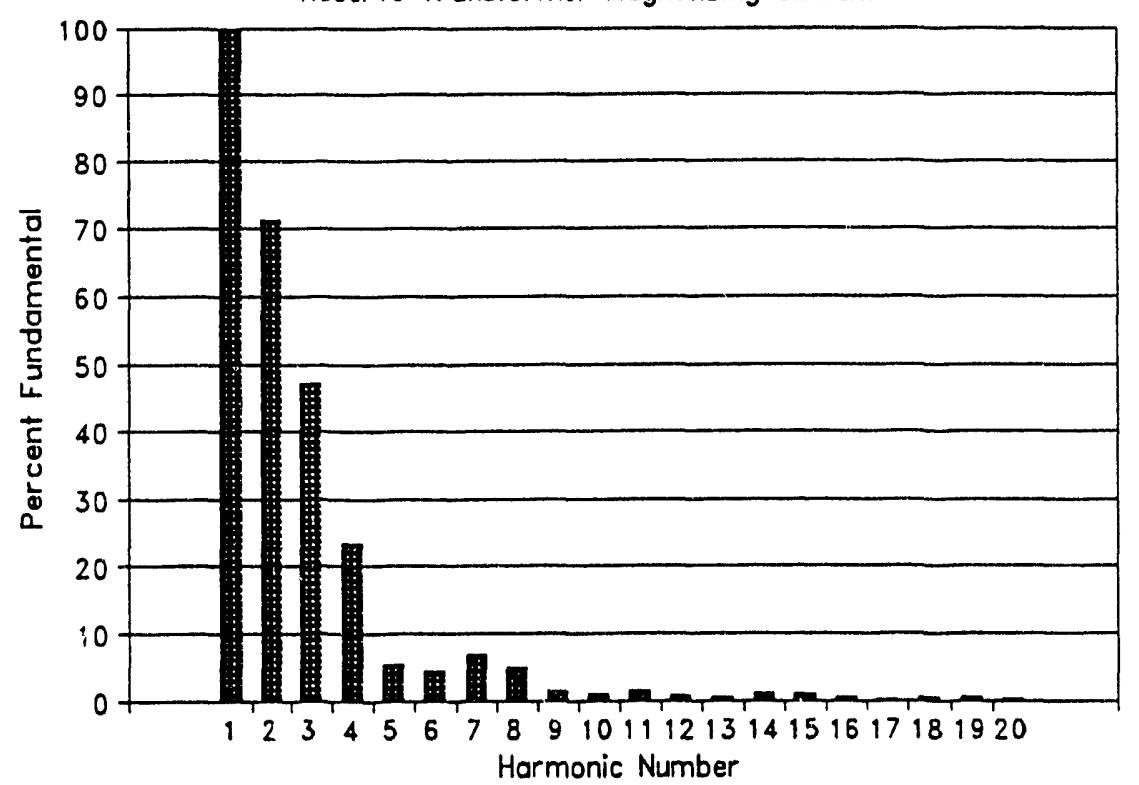

Fig. B-24. Frequency spectrum of reserve station service transformer magnetizing current. 


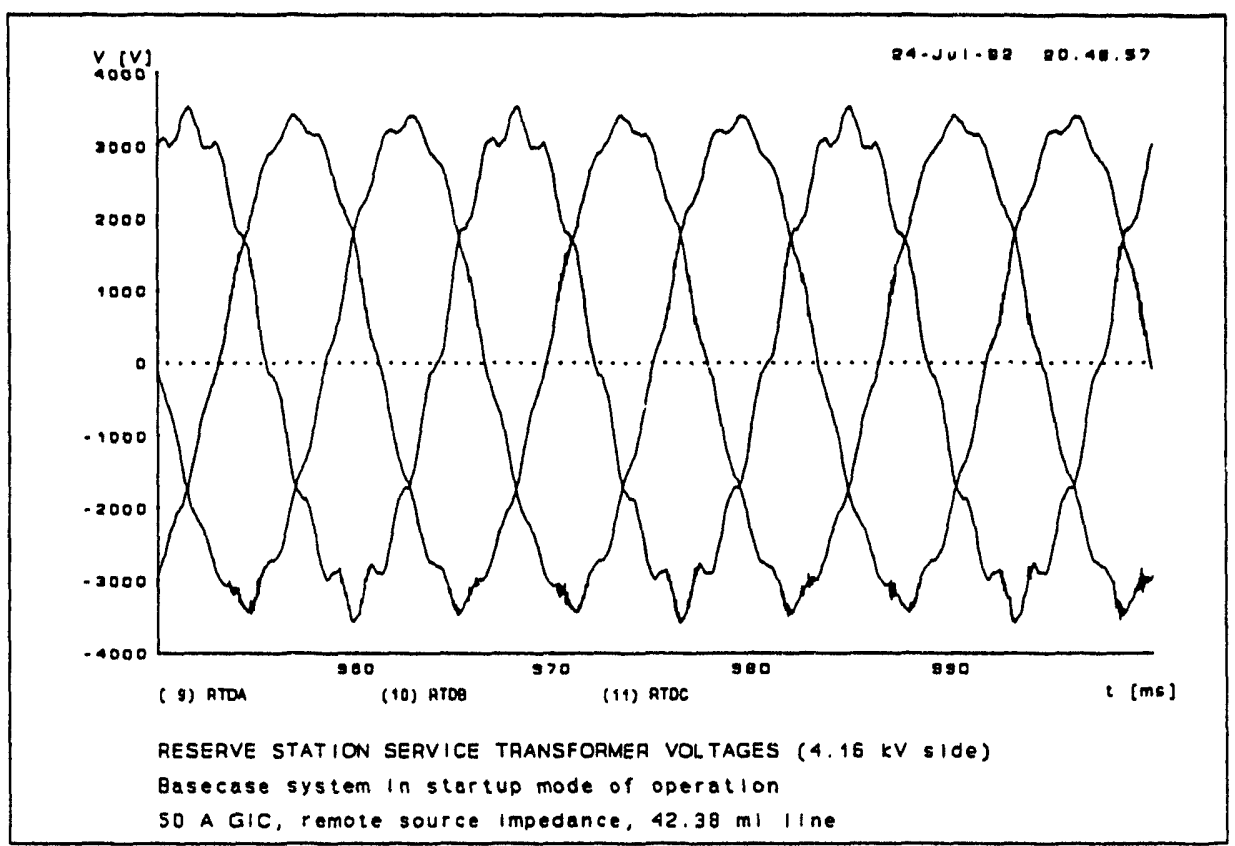

Fig. H-25. Reserve station service transformer voltages on 4.16 $\mathrm{kV}$ side during start-up mode (50 A GIC) with $42.38 \mathrm{mi}$ line and remote source impedance.

FREQUENCY SPECTRUM

Reserve Transformer Voltage (4.16 kV)

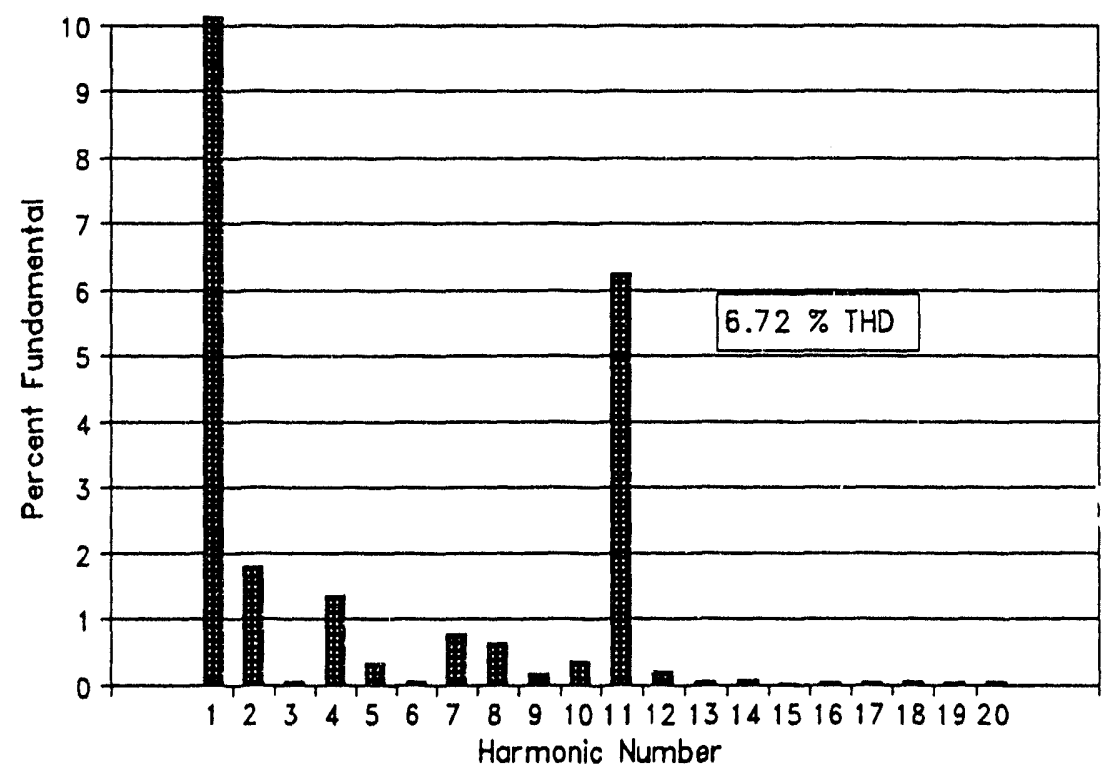

Fig. B-26. Frequency spectrum of phase A reserve station service transformer voltage on $\mathrm{LV}$ side $(4.16 \mathrm{kV})$. 


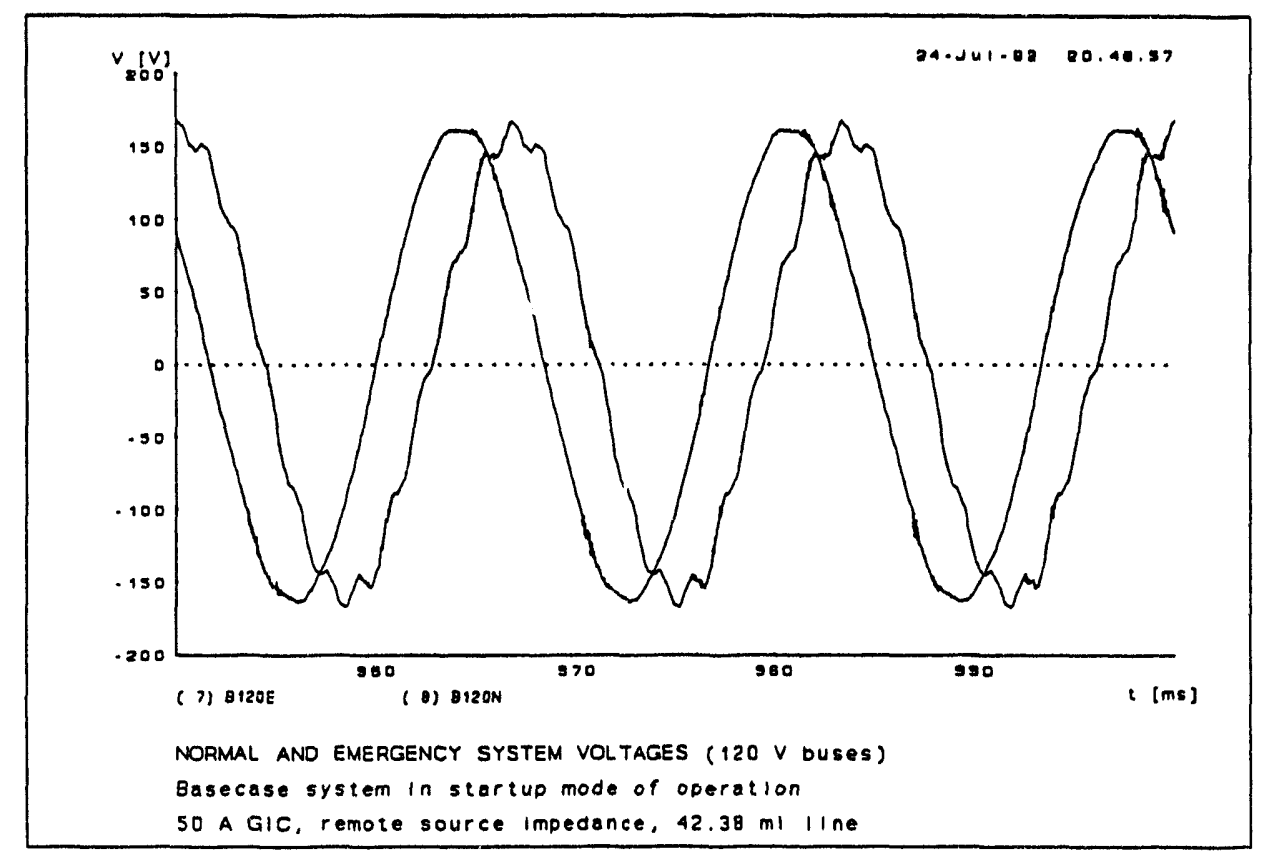

Fig. B-27. Normal and emergency system voltages at $120 \mathrm{~V}$ buses during start-up mode (50 A GIC) with $42.38 \mathrm{mi}$ line and remote source impedance.

FREQUENCY SPECTRUM

$120 \mathrm{~V}$ Emergency System Voltage

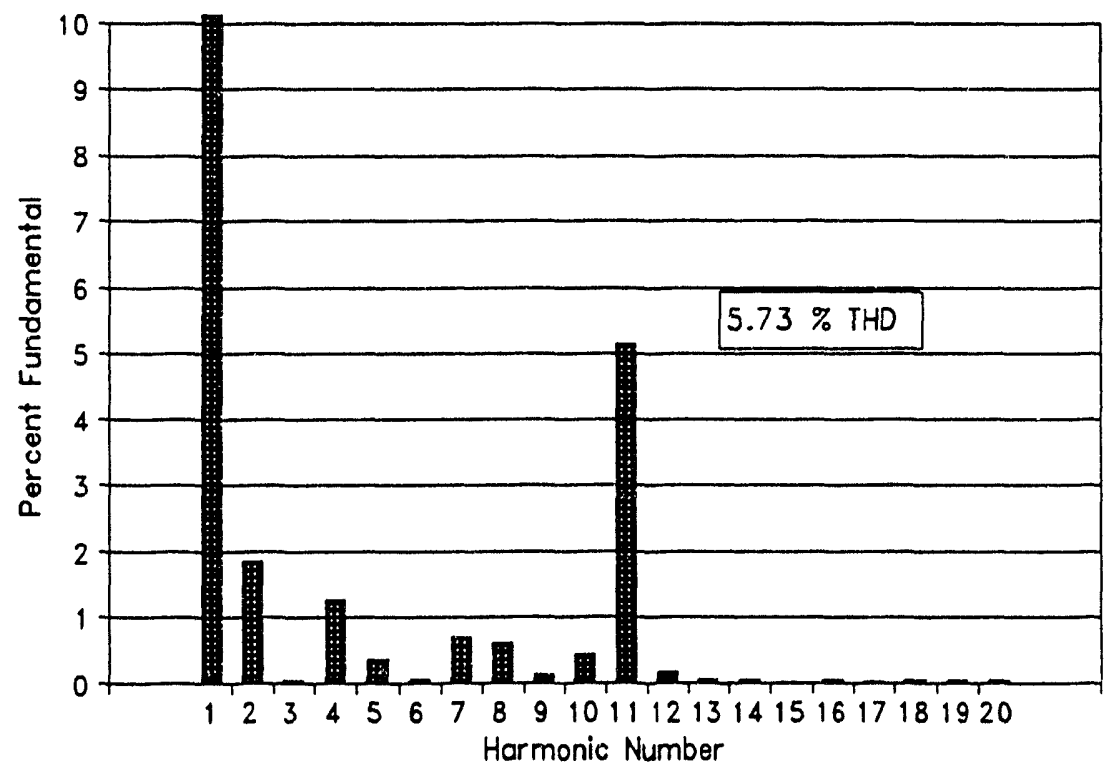

Fig. B-28. Frequency spectrum of $120 \mathrm{~V}$ emergency system voltage. 


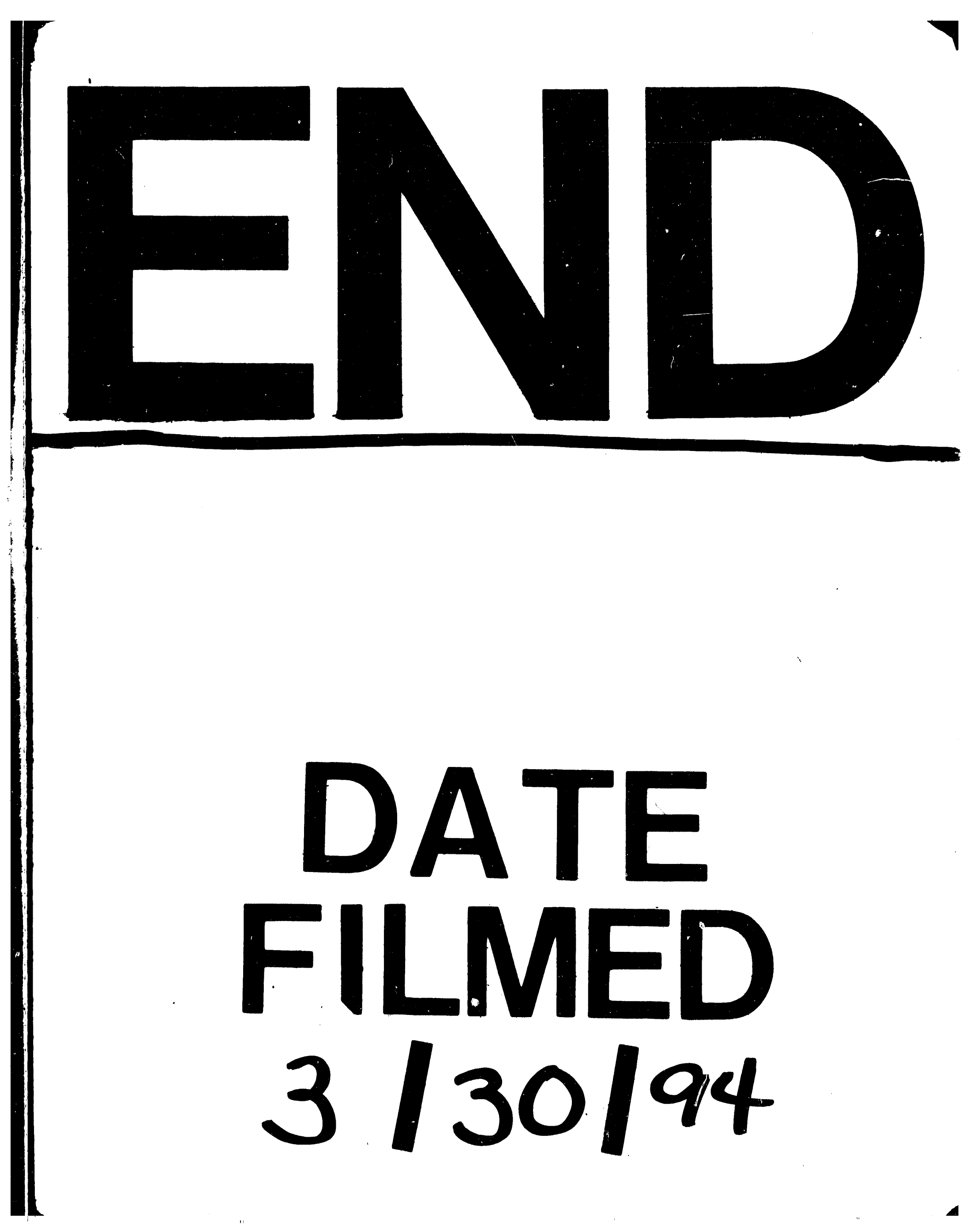


